

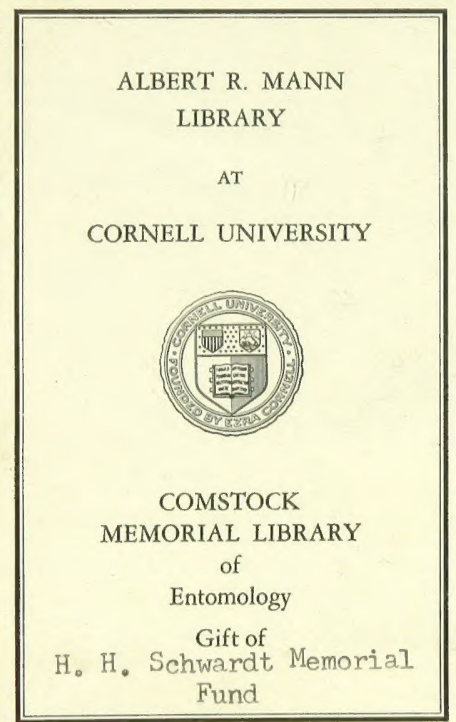


$11 / 15773$

DATE DUE

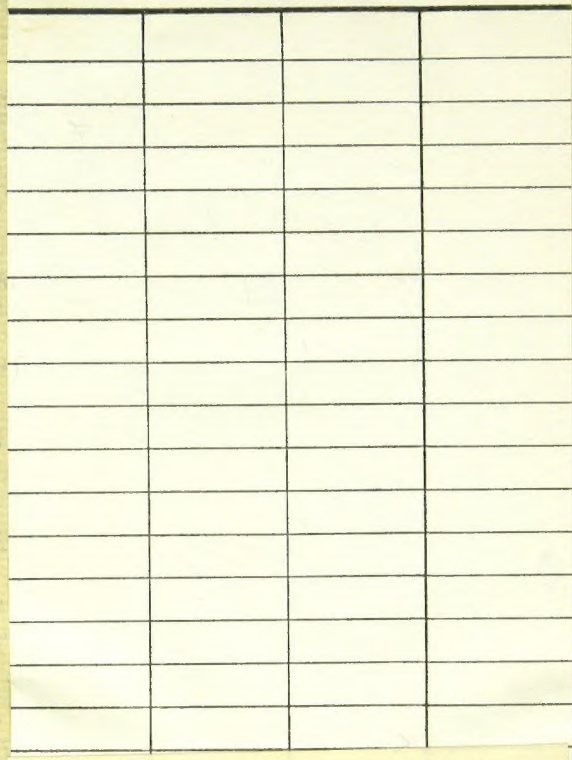

RETURN TO

ENTOMOLOGY LIBRARY

Cornell University

Ithaca, N. Y.

Cornell University Library

QL 561.R47W19

Skandinaviens dagfjarllar,

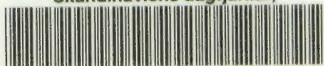

$31924018 \quad 317 \quad 317$ 


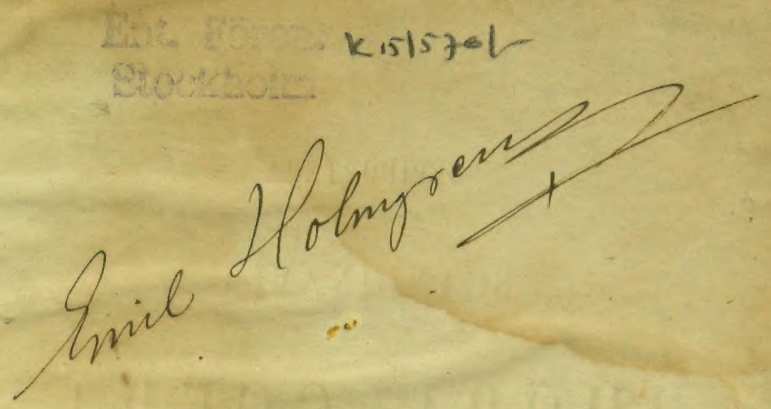




\title{
Lieproptera SCANDINAVIE
}

\section{R HOP A LOCERA,}

DISPOSITA ET DESCRIPTA.

\author{
A L CTURE
}

I. D. J. WALLENGREN.

MALMOGIN,

LX OFFICINA CRONHOLMIANA,

1853. 


\title{
Sfandimavieng
}

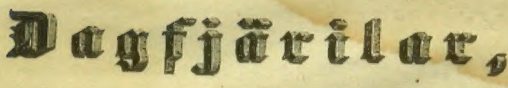

\author{
beffrifne
}

af

5. D. Э. Tsartengren.

SZaโmö,

tract hos 28. Exunbolm, 
QL

561
247 \&

w 19 


\section{Hans Hxcellence}

\section{Högvälborne Herr Grefven}

\section{G. Trolle Wachmeister,}

En af Rikets Herrar, f. d. Justitiæ-Kansler, Riddare och Commendör af Kongl. Maj:ts Orden, Ledamot af Kongl. Wetenskaps-Akademien,

egnas detta arbete

Vordradsfullt

af

Porfattaren. 



\section{สุ์}

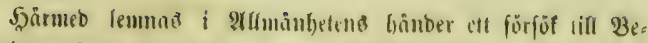

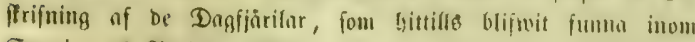
Swerige o(d) Morige. Mångn äro be brifter, fom miolion betta

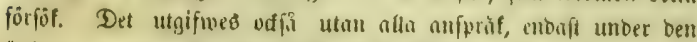
ünffan, ntt bet måtte tjena tia en banblebring fir m)(x'gl)mmuren

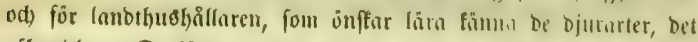

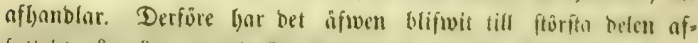
fattaot på mărt eget fprăf, ifumuerbet fom det eljeft, foreträbcos. wio of författare $i$ Entomologien, brufade latinet gôr arbetena otillgăngliga for ben ftörre mängben of bem invm mărt lano, fom hufa băbe wilja od luft att egna lebing ftuliser it maturems fubium, ods ajwen till en bel bafrom beloci of alt famm be finbliga od nbttiga infeftarterme, ment ife fortita bet leggngunabe

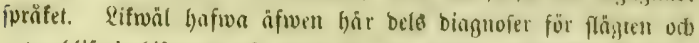
arter blifwit bifogabe pon latin för att gijma utlänoffin (Emtonto.

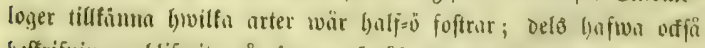
beffrifningar blifwit på fammu furâf nffattude för be arter, fom

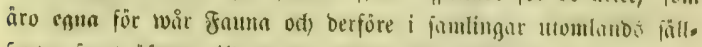
finto, faunt äfwent tifl en bel ofando efler misfindon.

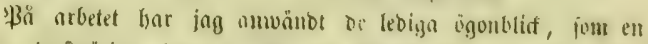

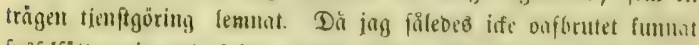

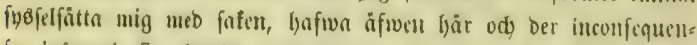
fer injuntgit fïg, huvilfa torbe benäget ofwerfes ods urfäftns. Till 
följe af trudningeortens afliggenlyet od beraf upwfommanbe hin=

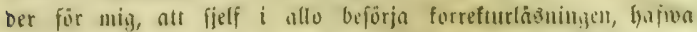

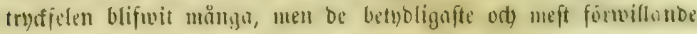

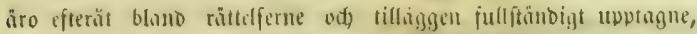

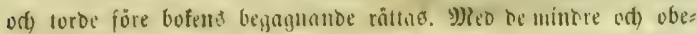
tyoligare torbe ben gunftbenaigne läfaren ofroerfe.

Det wore wiฮ̧ferligen win afitijt att fî fmonningum lemma

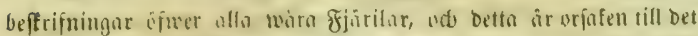

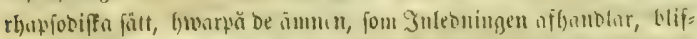
wit fromftallon; unen inmon en fïbun önffun fon renliferse, bes

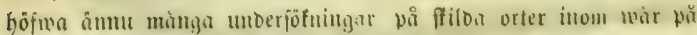

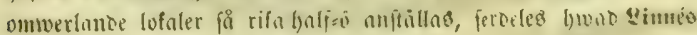
Noctua orb be juäre Fibilarne betriffor. Eă wioftridfu uns

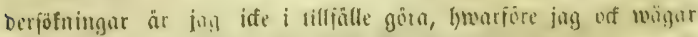
bemftaflo sill bem, fom intresiern fig för fofen, att antinģen fri

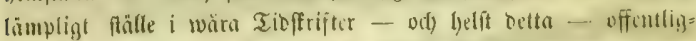

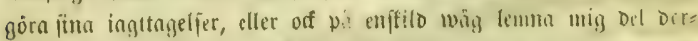

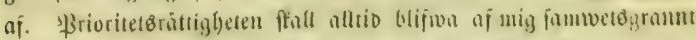
refpefterno. - Dwifheten furumidon min biar ofman utalabe ön=

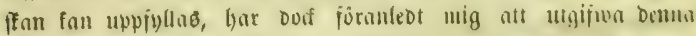
Beffrifuing under ferffilo titel, ifunnerket fou be bär afbanolones fiärilarterme biloa ett leelt för fig.

Jang fan ourjemle ife unberlăta ut bä Dffentligen uttofa

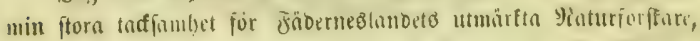

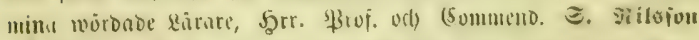

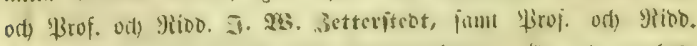

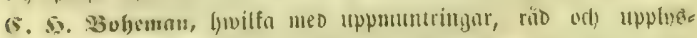

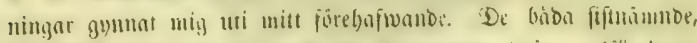

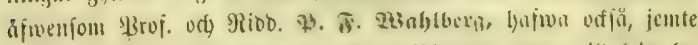

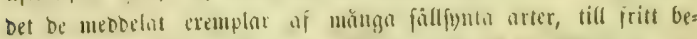

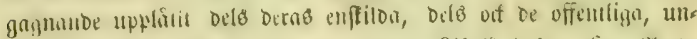

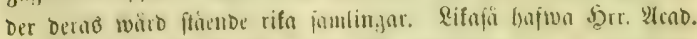

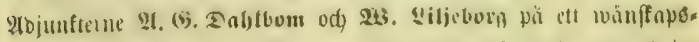

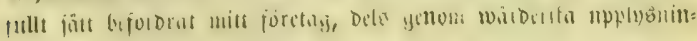


gar, bele of genum mebbelanbe af exemplar. Frin fir. Thool.

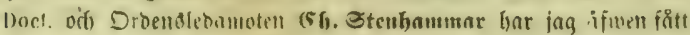

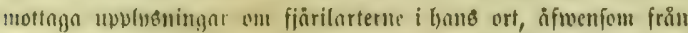

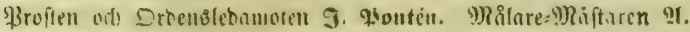

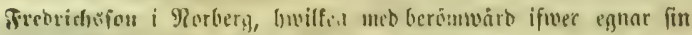

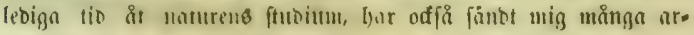

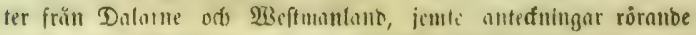
berne flugtio m. m. Dertifl att jain, fi wibe mojelipt warit, fun-

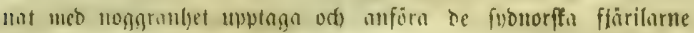

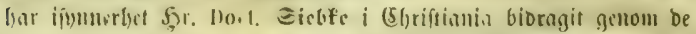
ontefningur, foum fon af intreofe för infen ftifft tifl mis bispes

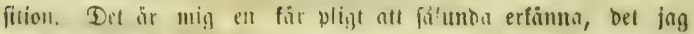

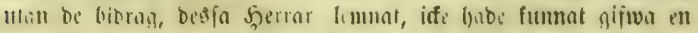

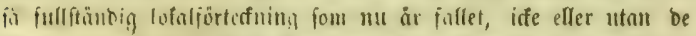

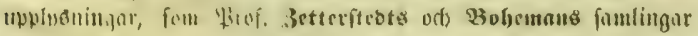
nimuit wio f)mbels, fumtul reba mangen intraplab frägn uti art:

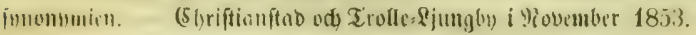

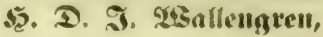

S. M. Adjuntet. 



\section{Förteckning prô arbeten som blifvit i Synonymien anförda :}

Mohem. - Hoheman. ilversigl al kongl. Wot. Acad. Förhandlingar 1847.

Roist. - Iolsduval : Icones Historique des Lépidoptères d'Europo nouveaux ot pou connus. Paris 1832-1838.

Borkh. - Morkhausen ( MI. IB.): Naturgoschichte der Europeischen Sclımetterlinge. Frankfurt 1788, 1789.

Dahlb. - Dahlbom (1. a): Skandinaviska Insekters Skada och Nytla. Lund 1837.

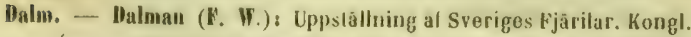
Wot. Acad. llandl. 1816. (Anfördt under alkortningen: Pap. Sv.).

Esp. - Faper: Din śchmolterlinge in Abbildungen nach der Natur mit Beschreibungen. Erlangen 1777.

Vabr. - Fuloricius (J. C.) : Entomologia systematica omendata ot aucta. Hafniæ 1793. (Anfördt under förkortningen : $E_{0} \cdot S_{.}$).

Fod. of Dup. - Godart och Duponchel : Ilistoir Naturelle des Lépidoptères ou Papillons de France. Paris.

H -S. - Nerrich-Schäffer (G. 1. W.) : Systematische Bearboitung der Schmotterlinge von Europa, ats Text, Revision und Supplement zu Hübners Sammlung ouropoischer Schmetterlinge.

Nïhı. - Nïbner: Sammlung ouropoischer Schmettorlinge. Augsburp 1805 .

hefeb. - Lefebur (A.) : Annal, de la Soc. Entom. de Fianco 1836. 
Liı. - Unur (c.): Fauna Suecia. Ilolmix 1761. Str.temil Nature. Fa. 12. Ilolmix 1767.

Mïll, - Mïller : P'rodromus \%oologie Manicie. Haluise 1776.

Nickerl. - Synopsis der l,epidopteren Pauni Roilımens. I'ra!; 18:30.

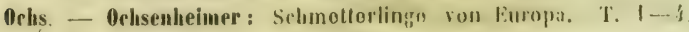
Lepzig 1807-1816.

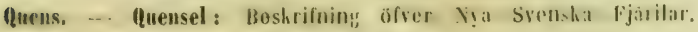
Kongl. Wet. Acarl. Ilandl. 1791.

Rumb. - Rambur \& Fauna Andłalousica.

Ross. - lless: Appendix to the Narrulive of a second rotalg:" in Search of a Norlh-Wust-Passage, 1835.

Schrank - Fauna hoica. Jngolsladt 1801

Srriba - fournal fur die Liobhaher der Entomologio. Fianlifurt 1790.

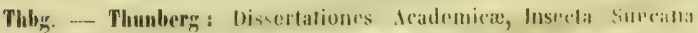
sistentes. Lualioe 1784-179\%.

Treitsph. - Treitselike : so Uchseraleimer.

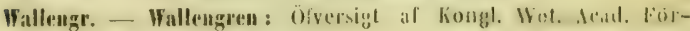
handlingar 1853.

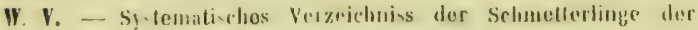
Wiener Gogend. II is: 1776.

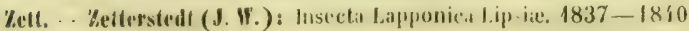

\section{Namnförkortuingar vid lokaluppgifterne :}

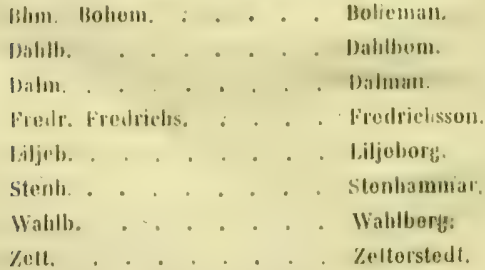




\section{In}

Tiourtlarue (Lepidoptera) anics wnuligen iafom lierbe oronin. gen af Soljefterna flora od astrifa tlaje. De bafma $\mathrm{i}$ allmänlyet fora binagtiga wingar, jom än foräfta meb jiäll, orbnnbe uti tats tegeliormigt lagba raber od falfftlande wid twingen mebelift en liten flielf. Nảgra få artere b,onor fafua ringar, elter baîwa blott rubi= menter Deraf.

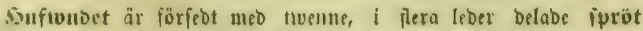
eller autemuer (antenna). Desija b. ftå ai ett fraft (scapus), fom

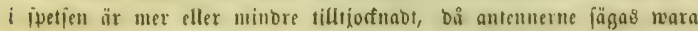
Hubbformiga (chavate) cller funqpiorminga (copitates). Sleffwa tilltiofningen fallab flubba (clava). Men be funna aforen fafua

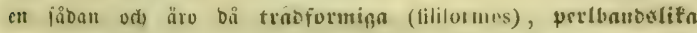
(monilitormos), allt ejtcriom leberue frumstäba mer efler minbre tholiga. Sallan äro de torfitformina (solacere). Unber alla besia

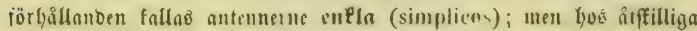
alter hafma be bwarielianba bilyang på fidorme od) benämmaอ̊ bå immanintta (compronite). Desia bilyang funm bels bajwa form ภํ tänderne $i$ en fam vd) antennerne jägå då wara fammabe (peclinates), bels af frålau $i$ en fiäber, dả antemuerme fallå fiảbrabe

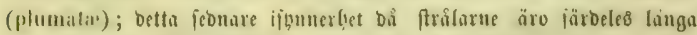

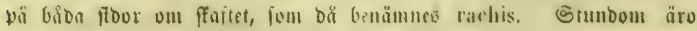

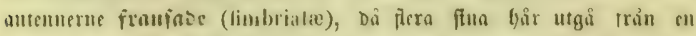

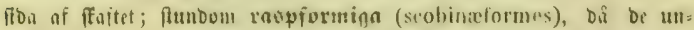

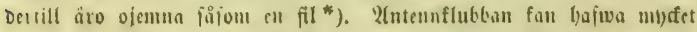

") Saman forgällanbe intriffine lifwal ef alltio lows biba fousen. 


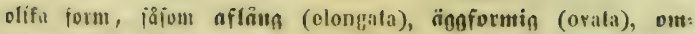

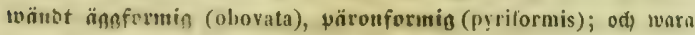
iperjert trubbißß (olifusa), rumbat (rofundato-obtusa), füriebo mes tage (mucromatia), eller fürieso mes frof (hamafa). Den fan of twan fammuntrudf (comprossit), ntholfno (oxcavala) จ. i. w.

Siutbelarme äro üh defamun hos ijärilarme fom lyos öriga

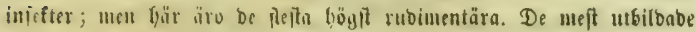
äro tungan o(f) palferme. Smanan (lingua) beftå of nwemue fuge rör, fom till bila längben äro Goplinganbe, fä att ett trebje bilbas mellan dem bảos. Det ät egensligent maxillerme, fom äro ombilbabe till tum 3s, fuxilfen mrerenbels är ganfe lang, men flumbou fort od rubimentär od) (1,03 niozra belt od) billet felflagen. (5meban ben of = taft ligger fammanullad i platt fuigcliom, fallas ben ipiraltumgn

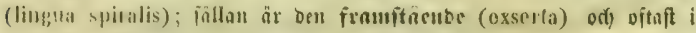
fpetien tunflufwen (bitida). Balperne ăro hod glärilarne egents ligen furis, fällon lix, men pitaft äro flott twenue labialpalpes

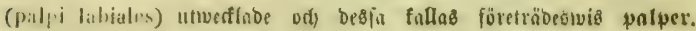

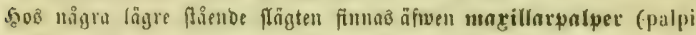
masillares) gamffa mųcet utwerflabe, umber bet att några bercmot nlloeles jafna walper. - Ditaft äro palperne trelebabe (triarticulati), jätlan tsualebas: (biarticulati), bögft jälan ferlebnbe. Till betne form âto be bels frå ftoorne fonmmutrycfita (compresi), od) bele plattructita (depressi). Dita äro be framitâtnor (porrecli), uppatitizunoc, (adscendentes), tillbafabojon (rafl-xi),

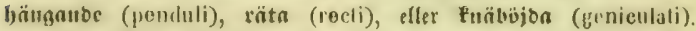
3 allmänbet äro be breon, men igumerbet los motten antaga be juä:* enre former, fio att be blipma trábformiga o. i. w. I anfeenbe till l'cfläbnaben äro de fï̈lliga (stuamati), hritfrmufate (fimbriati), peuílslifa (penicillati), o. ケ. w.

S̈guแen äro yoo itárilarne merenbels endaft jamumanjatta, แät=

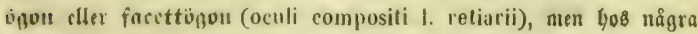

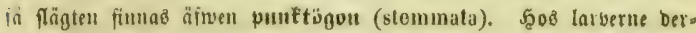

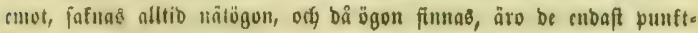




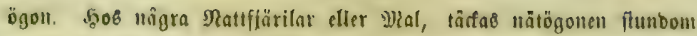

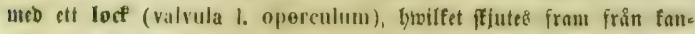
ten of uellantroppen.

Micllanfropucu, hos huilfen wisjerligen be wanliga belarme Punna urffiliab, men, med unbantag of mellauriugen (mesothorax), minbre utwed labe, är ofwan $i$ anleenbe tifl ruggen eller rybglpüben (dorsum) rumbab (rolundatus), ה̈ngforntị (ovalu.), flotformin (glohosus), fammantryeft (compressus), bön (altus) eller plattab (depressils). I anjeenbe till bariglieten är ben tätluoen (tomentosus), thocu (hirsulus), fórjios nted fum (cristatus), tuluobo (dontatus) o. i. w. Llubre belen af mellanfroppen fallas brüitst (poctus), odi) på rbgofölben urffilier man Galsfrą̧eu (collaro), fom omgifwer eflex är närmaft fyufwubet, oft) ffulorn (humerus), fom är närmaft wingbafm. - 23io mellautroppen bufrea wingarne ở) fötterne litt fäfte.

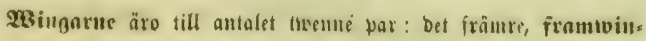
garme (alie antice, I. anteriorms, I. primores), od bet bafre, bat: uningaruc (ata: postica, I. posteriores, I. sucundatie). Till for. men -äro be Iñmgitträctto (olongalie), Inucettformign (lanceolate), runoabe (rotundata), o. i. w. Wierendels äro de obelabe (indi-

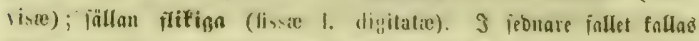
pifarme: digiti. $\Im$ amjeenbe till utfanteng beffaffenlet äro be bel*

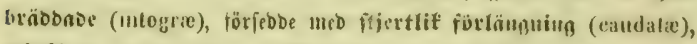
winflade (angulate), imatanoade (denticulate), od tatsonde

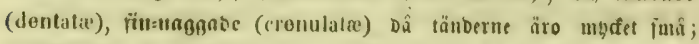
inrgtanonbe (arose), bå tünberme äเo ftora od) ojemma; utffurun

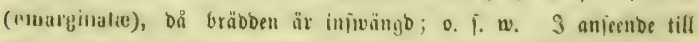

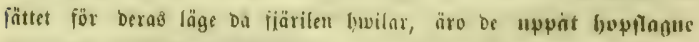
(orecta), plattliganoo (palule, I. pillentrs); utiperrabe (diva-

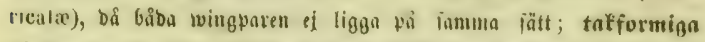

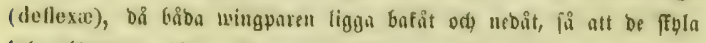

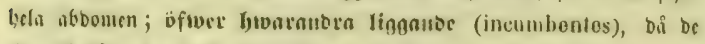

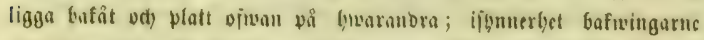

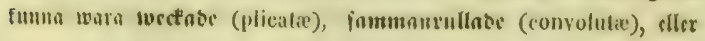




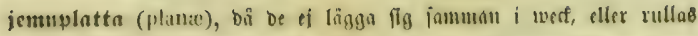

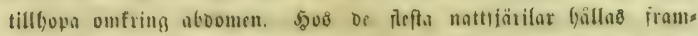
ninganne tilliamman meb Gafwilngurne genom en egen apparat (ro-

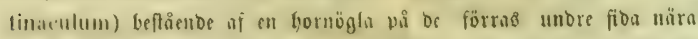

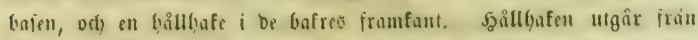

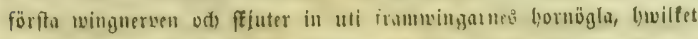

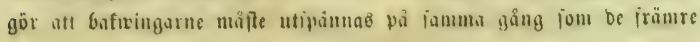
ord) Deltaga $\mathrm{i}$ besfą rörelíe.

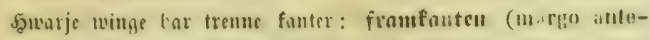
rior I margo), builfen på irommingarne ar en orbenligen urjeäno

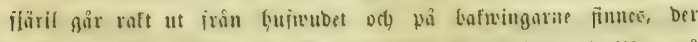
Desja råfa frammingurne; intunten (m. Fgo interior), burifen på

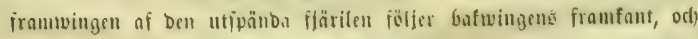
wă tufuringen iöljer nedomen; utfan tea (marg̨o oxterior I. posturior I. limbus), fuxilfon tå buarbera mingen camunabinber irams fantun ody infauten, fant är iörjeod med franine (limbria I. cilia).

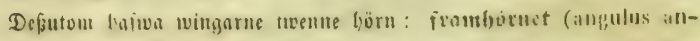
(orior), luniftet pá irammingane manligen tallas wisepipetien

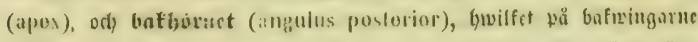

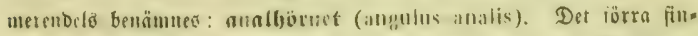
urs ber framfant v(t) utfant, Det jeonare ber utfant od infant möto lmuranora. Den bel of uxingen, fom är narmaft mellanfropten, fals

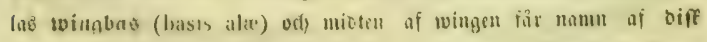

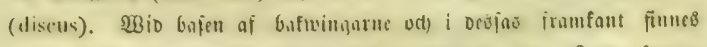

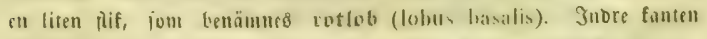
uf famma usinga bildor ofta offiả oll plif, furillen fallad absomis unlfite (lobus abdominalis). Di berma ffiuter nod omfring nboos

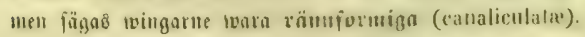

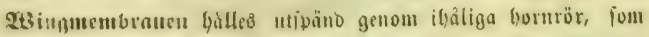

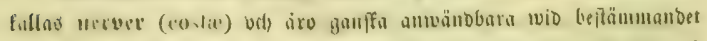

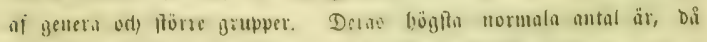
man bitratsar bem wio beraz utgảng irun svinghajen, egentigen fer. Debja äro: framfantonerven (rosta cosalis I. maryinis antorioris), bwilfen löper uti flelima framfanten; inbcoitaluerven (custa 
- ulicostalis), fon utgât frân taien närmnft föregienende od vitaft ut=

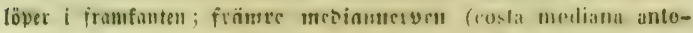

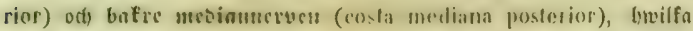
båba srio fajen äro furaranora temfigen närftâenbe, men unber lops

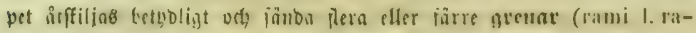

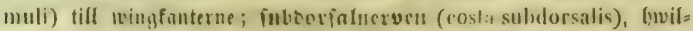
fen oftull utlöper i wingens bafre förn, men ftumbon uti benus ine fant, ofl) änotligen sorfolyerven (cosla dorsalis), fom altitb uttö.

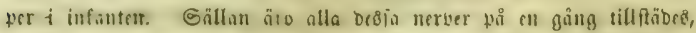

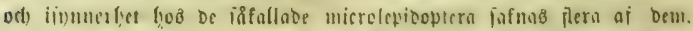
- Bába mebiannerberme inuefluto fia menne floor ett fölt, busilfet

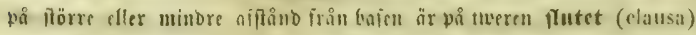
genem on terwery (cosla lransversa I. Iransversalis) od) fallab

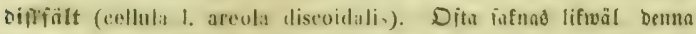

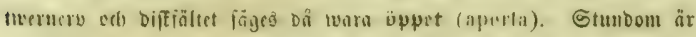

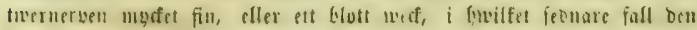
falla falp: (spuia); fandom orfia mer cller minbre afbruten

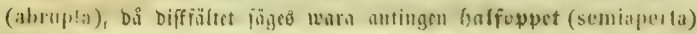

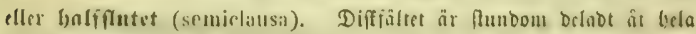
fin löngo medslit in fin nerw, hislpuery (costa auxiliaris), ellex

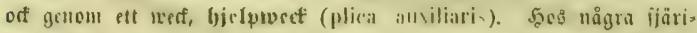

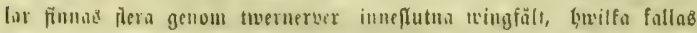

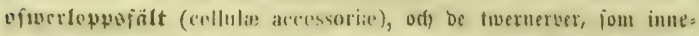

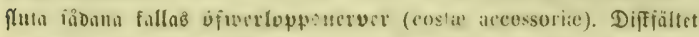

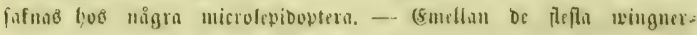

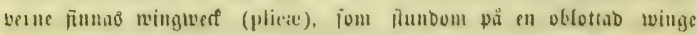
lifna nerwer, men foban angijällen blimeit sertrenjade wifa fig cas Dolf fäfom ionmar i membranen, der jiällen hait s"l omman fällning.

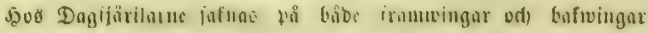

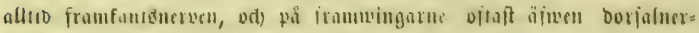

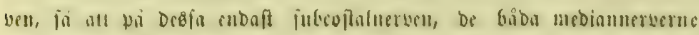

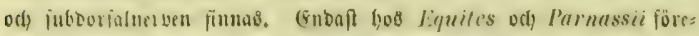
fomuler en fort borjaluerb, men los bedja grupwer fiffusb bercmot

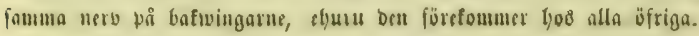




\section{XYI}

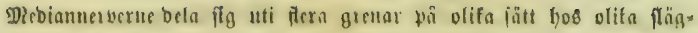

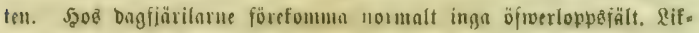
wäl finnes ett litet jäbant wib baien af framminganne emellan fubs

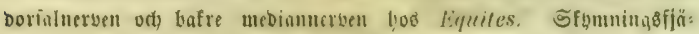

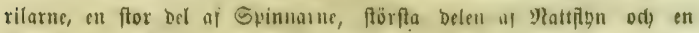

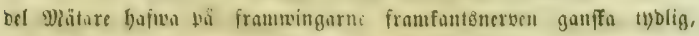

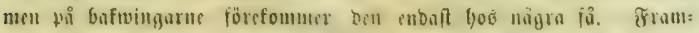

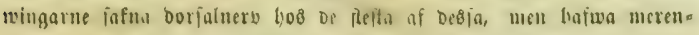

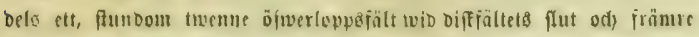

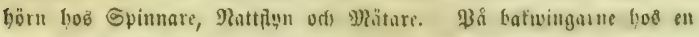

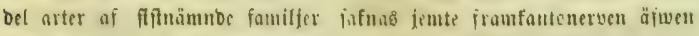
fubcoftalnersen, od) crfättes genom en gren af irämre mebiunnersen.

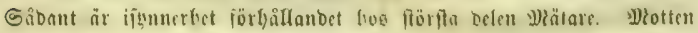

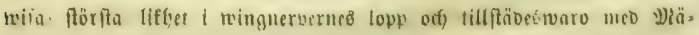
tarne; men loos be egentilgn microlepiooptera ât füshallande af varis

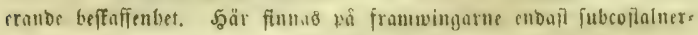

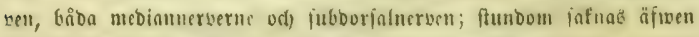

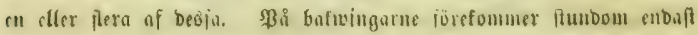
ena mebiannerben.

S efter fölianbe aflanding benämmas twingnerverne eftes beh me* $11) 00$ fom I a Iman föreflagit. Den räfnar bem på frammingurme frün infınten od ) på Gafwingarne från framfanten, od) Genänmes Dem

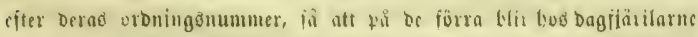
dorjalnervent eller, dä benna ife finteg, jubdorjaluerwen, vet? ba de

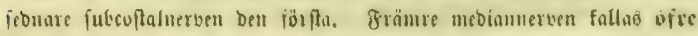

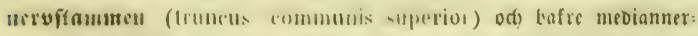

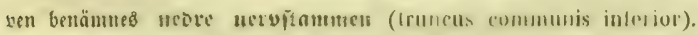
För unberlättandet mid gruppers od) generab indelning fât lifwäl Degja biba nervitammars grenar blort orbning8nummet fäfom ncrecr,

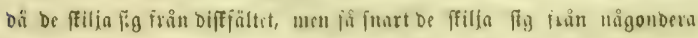

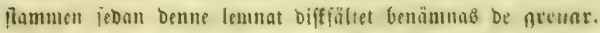

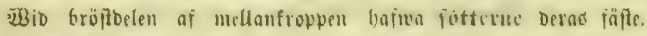
De äro till antalet jex, men de twenue förfta äro fros en bel Dasffä=

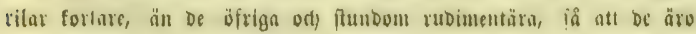




\section{XVII}

obrufbara twib gienbet. Derab belar äro be fanme cont loo anbra infefter.

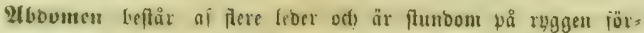
lébb med en ellet flera bărfommar, då ocn fäges waı betofias

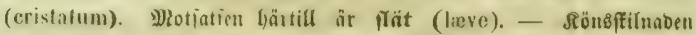
wifar fig till bet yttre beri, utt lannens analöppuing merendels är omgifwer af en mer eller minbre biwig garborfle (anallorfte), buil=

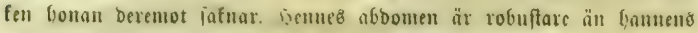

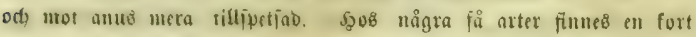
bttre äggläggningฮ̊fliba.

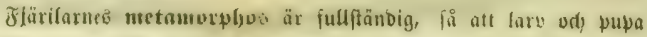
äro finsemellan till formes belt vlifa, äfweniom olifo med ben full= bilbabe injeften (imago).

Q̈ ffilba genera och arter. Ŝlı äro be rumba, elliptifa, ffáliormiga, $c h=$ linbrifta, fegelelifa, lyalirunda cller vetiolerabe; ân äro be refîlabe, ftrierabe, dagrinerabe, punfterabe, taggiga, hårfranjabe sller fläta. Ltaf mubren läggaв de altio på ben planta, fon ffall ticula ben "Igfläafta larven till uäring; men ber funua be än lägga iptibba, än $i$ bögar, fator eller ringur. Ditn omgijuas be af flem, fom feall

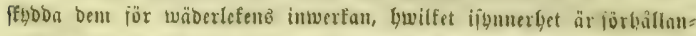
Det hos be arter, bwars ägg ffola uttläfas förft paiföljande wir.

Larven (larva) är hos olifa fảgten of olifa form: ofta (a) linbrife (cylindrica), ment äfuen afellformi (aselliformis), di bels lifnar till formen ell wanlig gråjugga fniß̨f formiß̧ (limacilormis), ipinbelwriosu (verticillati), oval (ovalis), affunaluanos (altenuafa) och tillipetiob (acuminata). Till ptas är ben uaten (nnda), bariß (pilosa), Inbou (lirsuta), borfitin (lasciculato-pi-

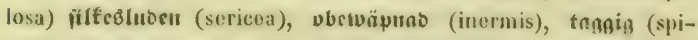
nosa), behoruno (cornua), teutafuletas (Lentaculata), wärtiß (verrucosa) cller füufnulig (luberculata). Rarverne lefwa antinģen

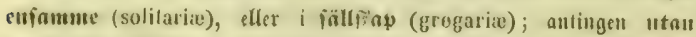
bitäctuin@ (diales I. subdiales) cller meb betäffring, i blvilfet jeb. nare fall be funna wari bele inwedtabe (involuta), då be omgifna 


\section{xvil!}

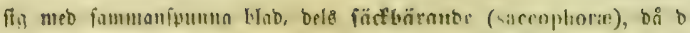

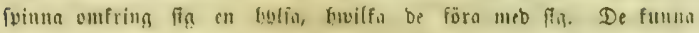
änen lejun solse (l.tentres) antimuen uti forben, i räbene web:

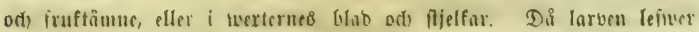
Dold uti waxterne, ber ben lildar ginngar i Waben eller weben, fal.

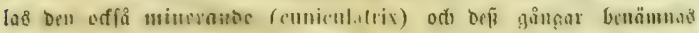
minor (rmiculi).

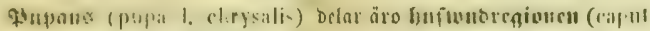
1. regio eapilis), mefloufroptstegionets (thorax I. regio thoracis). aboomitunlregiontea (aldomen I. resion abstominali-) ods win:

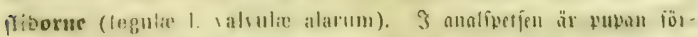

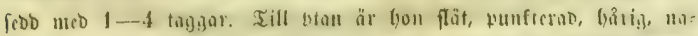

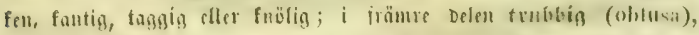

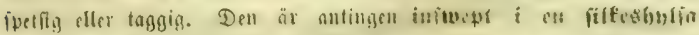

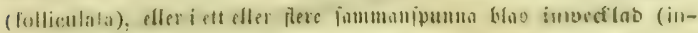

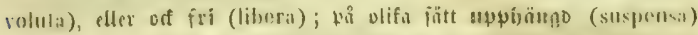

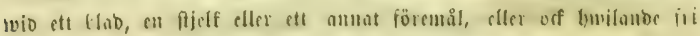
i jorben, $i$ mosia, unber flenar v. f. Iw.

Siffom $i$ ofriga 3oslogiens brlar bafma wi äimen bär Den

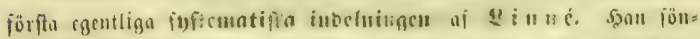
brabe fiärilatues oloning uti trenne flo1 od) artrifa ftägten: Dag.

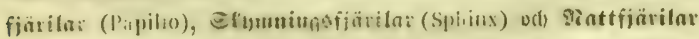

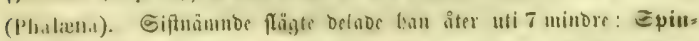

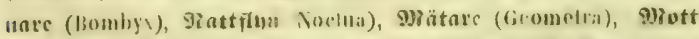
(1'yratis), Sticdtare (Jomil), Mat (Tinea), od) Finocrmott

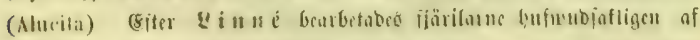
(5)

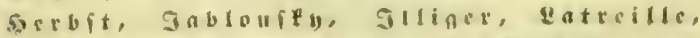

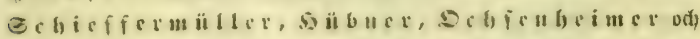

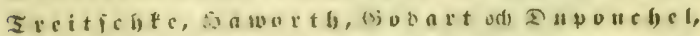
so

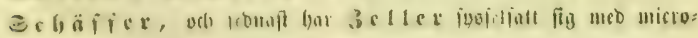


lepboptera od fortfar ast lemma ntmärfta monograplier öiner flägten

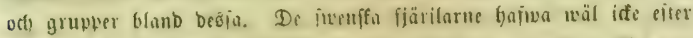

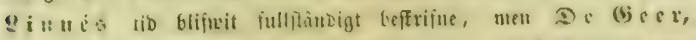

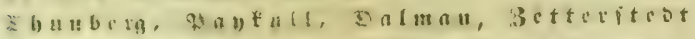

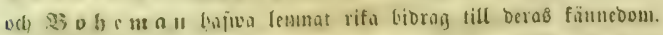

Sovelungen uti Ingfiütilat (1,opidoplesa dumna), Efume mingofiärifar (Lepidoptera crepu-cularia) od) Sinftfiätilat (Le-

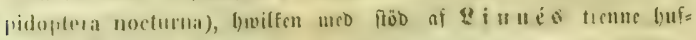

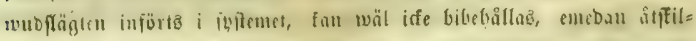

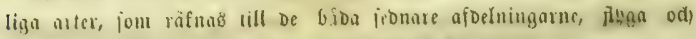
äro i rörrlfe un Dagen lifa mucfet fom nagon af of egentliga onge

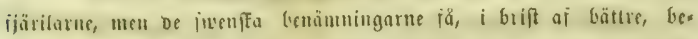
g)

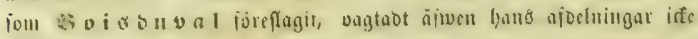

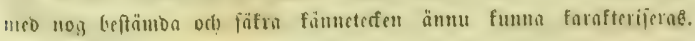
Des̆li afoelningar äro:

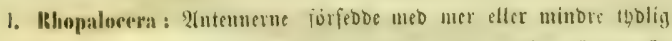
flubba; beras meblerfta leber mudet längre ān breba. 2tntingen Gåba wingvaren sller blott bet främre uแDet Gmilan merendels uppåt Gopflague. 3ngen. Gornögla tå framtvingarne us unbe f̆ba, clet Gålibafe i bafruingarmes framfans. ซ̌ram= fantônerben wå båbe franuvingar ud) bafuingar fafnละ; ofląt äfuen boljainersín på de förra, fällan på bc febnare. Aferig opftuerlopwsiält wio

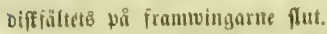

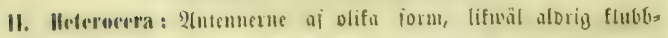

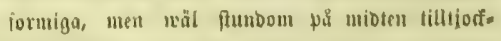
nabe; beras uederfta leber ef längre än bredo.

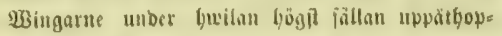
flague, utan untingen tatiormign, plattligganbe, utiperrabe, ofwer fuvarandora liggande, fomman. rulabe eller twedace. Merendelb jornügla på framuingarnes unde froa ods trillyafe $i$ bafwin. 
garnes framfant. Dfta förefimmes framfintosnere ben

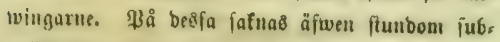
coftalnerben, ftumbom förefinues blott en eller twentue nervex. Dita ett eller twenne öfwerloppos jălt twio biffaăltete på frantwingarne flut.

Sill fürra afbelningen büra alla be făfallabe bagfin̈rilarme eller g* i u u e * flägte Papilio od till ben iebnare alla be ôfriga eimés anffa flägtena.

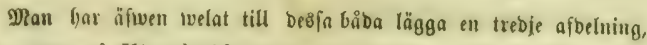
unber namu af Microlepidoptera. fammanfattande berunber Qi II: "ex s fligten: Tortrix, Timea o(f) Allucill, men benna berảmnis fan enbaft lyafwa wraftiff nitta iåfom fortare än uppräfunabet a। flägtcna, ori) fan ife antuänbab $i$ ieftemet, cmeban ben enbaft afier bell minbre floriefen. 


\section{Lepidoptera Scandinaviæ.}

\section{Rhopalocera.}

\section{ERandinavicus Dagfinilar.}

Dngfiñrilarne utgöra förfta Gufwubafoelningen af \&lärilarnes Drd. ning od) fallas meb ett gemenfamit namu lkhopalocera, till fölie ai De entla, mer eller ninbre flubbiormiga antennerne. Namuet ăr fam. manjatt af be Brrfiffa orben: rhopalon, flubla, ody keras, forn. De äro $i$ rörelie om bagen orh Gwila unber natten, wen be lefta arter.

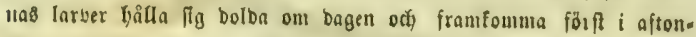
fọmningen att föfa fin fôba.

\&arwernes föboämnen äro fyufnubfafligen wexterneह blab od) ftäng:

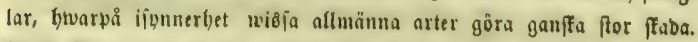
Snga Sfanbinavifta arter bämta fin näring of twerternes rôtter. \&̊ำ Satyroiderne bafwa gräfen blipuit anusifabe och benna famili enfamt omfattar nära ell trebjebel af alla fänba bugfiärilar. De öfriga fá millerna beremot föfa dicolyledonerne, eliuru, fenförclieevis meb Spinnarme od ilunnerlyet med wisia familier blanb mictolepidop-

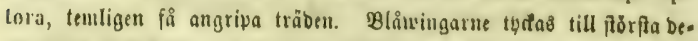

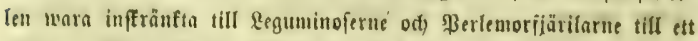
enba iveriflägte: Viola. 24nbra iter lefwa på flera wertarter od werts

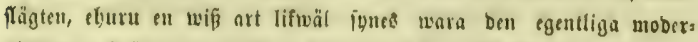

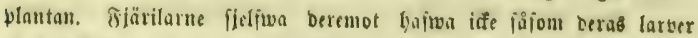
f̊̊ inferảnfta gränjor för föboämnena bămtanbe. Sgela floras rife ftir bem till fubs. Dffia begagna be fitg af benna fribet. Från blomma tilf blomma färoas de liela bagen igenom utan att trötma,

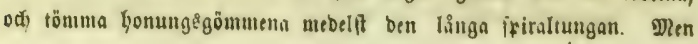


be förfafta iffe eller anbra wäffer, fápom bent faft, foul utfipprar ur

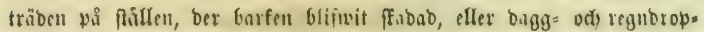

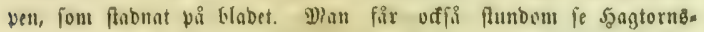

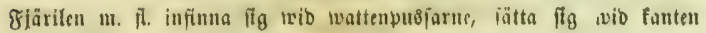
beraf odi begårligt uppiörpla nf mattmet.

(ङnligt föllande id)ema indela 2ffoelningen i twenue familjer: I. Fam. Papiliones. Margo anticus alarum anticarum ocostatus costis 2-4 extgnditur. Ex apice cellule discoidalis alarum posticarum aut costa nulla, aut distincta et normalis egreditur.

gramtanten of frominingarne, fom fielf jat: nax nerb, bålles utfuänb genom $2-4$ nerwer. Ur fpetjett ai bafwingarmes biffiält utgår antingen in. gen, eller od en toblig, be öfrige lif nerb.

II. Fom. llesperioidr. Hargo inticus alarum anticarum ecostatus costis 5 extenditur. Ex apice collulæ discoidalis alarum posticarum costa nulla, sod plica subtilissima loco costæ egreditur.

Eramfanten af frammingarne, fom fielf jaf: nat ners, hålleb utfpäno gentom 5 nerver. Ux ipetien af Gaftwinģarnes biffält utgâr ingen egent= lig nerb, utan $i$ beß̧ fălle ett fint wingwed.

\section{Ram. Papiliones.}

Margo auticus ilarum auticarum peostalus costi: 2-4 extenditur. Co-ta harum unira plerumguo plus milus ramosa, rarius omnes simplices. Ex apice cellule discoidalis alarum posticarum costa aut nulla, aut distincta of normalis egreditur.

Caput medincro I. minutum (thorace quidem latitudino sempor, altamon vario modo, minus). Antennæ basi approximatæ. Stemmata nulla. 
Corpus, respoctu habito expansionis alarum, gracile. Thorax abdomine laad latior, altus ot convexus.

Alae omnes incecti duiesentis erecte, ample. Margo interior alarum posticarum aut defexuz, ut canalis pro receptiono abdominis formetur, aut oxcisus, ut abdomon sil liborum, nullo modo tamen per tofam Iongitudinom plicatus. Collula accossoria nulla.

Larra subdialis, 16-poda, ẹlindrica, olongata, aselliformis I. subovata, vario modo armata aut hirta.

Pupa angulata, rarius lævis; ant ano tantum, allt filo Iransvorso quoque alligata, rarissime sublolliculata.

Framfanten af franmingarne, fom fielf faftur nerv, bålles utfpäno genom 2-4 nerver. (5n af bes̊ja är merenbels ner eller min. Dre grenig, fällan åro alla enfla. Ur ipetfen of bufwingarne biffiält utgår antingen ingen, eller ỡ en thblig, De öfriga lif nerb. Intet opwerloppsiält.

Sufroubet mebelmåttigt eller litet (alltib, men på olifa jätt, $i$ nuje. ende till breboen minore än thorax). 2(utennerne wib bajen Givatanbra närftåenbe. Inga punftögon.

Siropqen meb Gänjeenbe till wingutpröåningen fmal. Tajorar föga brebare än abdomen, ligg orf fonver.

2Biugnane unber fivilan uppost bopflague, wiba. Bafwingarnes in: fant antingen nedböid, få att berigenum bilbas en rỉnta, fom upptager abbomen, eller of utff̈uren, fi̊ att abbomen bänger fri; men albrig på längben weđ̛ub.

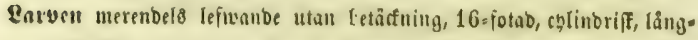

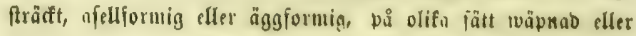
bårig.

Wupn॥ funtig, fällan flät, antingen Glott fịjtıb mib analtaggen, efler ouf beriemte mebelft en out froppens miot flagen träb, bögt fällan liggunde i ell fo:fonglif holja.

Till benna familf böranbe arter bafima tifl följe aj be, jemio. relienvis med froppen, flora wingarne en boppanbe Thgt, builfen ax mer eller minbre ibållanbe. Sn bel äro lifıảl ganffa inabopuganbe. 
Storfta belen äro $i$ rörelie från morgonen änba tifl aftonen, men eII bel blott $i$ mibbagsftunben. Några ăro äfwen fá fänfliga för jole wärman ody Geroenbe af benna, att be föfa ftybo ia juart moln forts ftommer folen. Desta är ifmunerlyet förbållandet meb naigra of be l)ögnorbiffa arterne. Bergiga, böglänbta ods iumpiga trafter hafwa f) warbera fina arter. 2̈fwen inom inöregionen finnos naigra repres ientanter af fantilien, fuilfen man inbelat $i$ feftioner od, grutuper, fom pฉ̊ follanbe fätt låta farafterifera fig :

\section{Sect. Tetrapodes. Dus.}

Podes tantum quatuor postici grossorii; antici duo spurii, broves, debiles.

silott be fura bafre fôtterna äro ganngfötter; De båba främpte falfta, forta, rubimentära.

Collula discoidatis alarum posti-carum costa transversa celeris æequali clausa. Margo anticus ularųm anticarum costis 3 et ramulis duobus quartre exlonditur.

Bafwingatne bifffält flutet gentom

I. Trib. Satyroidae. en twernerb, fons lifnar wingnerberne. Framfanten af jramwingarne bålles i utfpảnb genom 3:me nerver odi) 2:me grenar af en ilerbe.

Cellula discoidalis alarum posticarum plane aperta L. costadransversa spuria subclausa. Margo ànticus alarum anticarum aut costis 2 et ramulis duobus tertix, aut II. Trib. Nymphalides. costis 3 et ramulo unico quartæ extenditur.

Batwingarnes biffält albeles öp. pet, eller blott tillflutet af en falft 
twernerb. Framfanten af framwin. gurne प⿺⿱乛龰) 2:ne nerver odi) 2:ne grenar of en treble, eller of genom 3:ne nerver odi) en gren af en fierbe.

\section{Sect. Illexapodes. Dan.}

Pedes 6 omnes gressorii, invicem conformos.

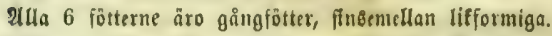

Cellula discoidalis alarum posticarum costa transversa, re-
liquis equali clausa.
Bafmingarneo̊ diffält futet genom en twernerb, fou litnar
wingnerberne. Margo anticus alarum anticarum costis 3 of ramulis aut duobus, aut unico quarte, rarissime costa unica of ramulis quatuor secundxe extenditur.

Framfanten af framwingarme Gåls > III. Trib. Ileliconides. les utipänb genom trenne nexber od) genom 2:ne, eller blott en enba gren of en fierbe, bog fâtlan ges nom en ners ods 4 grenar of en anbra.

Marge articus alarum anticarum costis 3 simplicibus exten-
ditur.

Framfanten af frammingarne bål. IV. Trib. Parnassii. les utipäno genom 3:ne enfla nerver.

Margo anticus alarum anlicarum

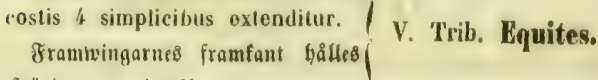
แโpäแb genom 4 enfla netber. 
2. Cellula discoidalis alarum posticarum aperfic, plicat trans-

Margo anticus alarım àntica-)

rum costis 4 aut simplicibus, ant

una biranosa oxtendilur.

Framfanten uf framuvingaıne bål. VI. Trib Lycenoidar. les utipand genom 4 nerber, antin. gen alla enfla, eller ben erta twå grenig.

\section{Sect. II eteropodes.}

Pedes 6 omnes fominte, \& tanfum postici maris gressorii : antici duo maris breves, debiles.

2lla 6 föttcrna hos honall, then blott be 4 bafre hoo byannen äro gångfötter; be båba främure hos fnnuen forta or. rubimentära.

Cellula diccoidalis alarum posticarum co:ta transversa in modio abrupta semiclausa, Margo anticus alarum anticarum costis 3 et ramulis 2 quartw extenditur.

Baftringarmes diffält halffutet ge= VII. Trih. Erycinides. nom en i miden aforuten twernervo. Eramianten af framtwingarne bålles utfpäno genom 3:ne nerver od) 2:ne grenar af en fieroe.

\section{Trib. Satyroidae.}

Pedes tantum quatuor postici gressorii, antici duo spurii, brom ves, dobiles, sæpius pilosi. 
Cellula discoidalis alarum posticarum costa transversa cotoris requali clausa; anticarum costa transversa ant continua, aut plus minus abrupta, clausa.

Margo anticus alasum anticarum costis 3 et ramulis 2 quartx, nempe costis alie $8, " 9$ of 10 , of ramulis anticis costæ septime, oxtenditul:

foste alarum posticarum sumpar 9; la e teunco communi cum 2-1 egrediens el ramulum broviorem aut distinctum, aut tantum turviditate vario modo indictum, in lobum basalem emittens; 8-9 e basi egrodientos; anticarum 10 (septima triramosa), ad basin una of altera inflata; 1:ma e basi eyrediens.

Ilæ posticæ pro rocoptiono abdominis canaliculatie.

Larva eylindrica, inermis, nuda aut sericea, ano bimucrouato.

Pupa submutica, apico anali suspensa.

Fötterite: biots be fora fafle äto gångfötter; främfta paret forta, rubimentära, oftaft lubna.

Dipliältet på fraftringarme flutet genom cll thetuers, foum är lif wingaerveıne; på framwingarne flutet antingen genoun en full. flänbig, eller genom en mer eller minbre aforuten twernerty.

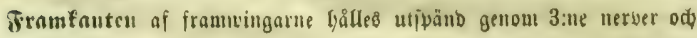
2:ne grenar af ell firrbe, nemligen genom 8:be, 9:be och 10:be ivingnerberne od de Gába främre grenarne of ben. 7:be.

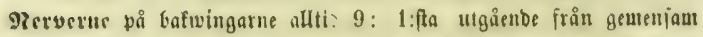

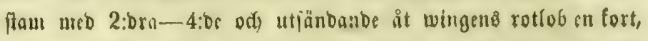
antingen tyblig, eller genom ell uppfuällning på olifa fätt an thbo gren; 8:be-9:be utgånend från Gafen. De frümres ner. wer 10 (ben 7:be tregtenig), ben ena od anbra wib bafen upp= blâf; ben 1 :fta utgienbe från bajen.

Bafuiugatue bilbande en rämun iör afoomens upptagunbe.

Earecu chlindriff, obewäpnab, antim\}en nafen eller filfesluben, meb tmåpet fab anนธి.

Pupan föga fantig, fnptyänganbe meb analtaggen.

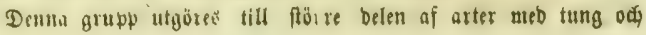




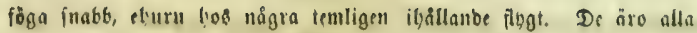
föga mångiärgabe od) lefiza făfom larber uteflutanbe af gräвurter. Den ffaba be jörorfafa as boef $t$ bet byela ofethblia. Sarberne lefwa om bagen bolba $i$ bräjet od) utgå förfi om aftonen att föfa föba.

Flera af bittöranbe atter gà ganfta högt mat norbent od) uppát

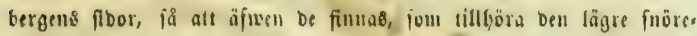

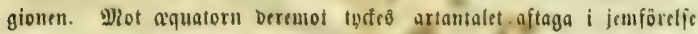
meb anbra grupper, och jpnes benna fileoes företräbestwib silltöra ten sempererabe oft beri arctipla zonen.

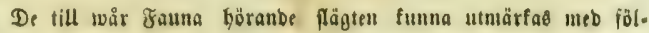
janbe torta farafterer:

Truncus inlerior, ut etiam costa prima fot costa docima alarum anticarum, ad ba-

1. $\{$ sin indatus. 1. Coenoнympha.

10:be nerv rib Gajen uppblåf.

- Truncus inferior, ut eliam "costa deci11. ma alarum anticarum, ad basin anilatus. f Eramivingarnes nebre nerbftami oda 10:be ners wib bajen uppblafte.

Costa transversa cellule discoidalis falarum anticarum ramulum brevom,

1. Tusermervelt wib frauniugarnes bift= II. Pararga. fält uriänoer i flelfn'n diffiältet en rit ody fort gren.

Costa transversa collulæ discoidalis

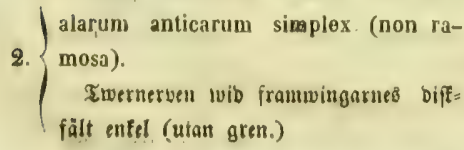


Costa transversa alarum anticarum intor costam 5 et 6 oblique subarcuata (Ramulus coste 1:me alarum posticarum tumiditate subquadrangulari oblusa indiétus. Intervallum intor costam alarim anticarum 8 et 9 triplo majus quam inter costam 7 a. ot 8).

III. Aphantopus.

Bafwing

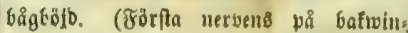
garne gren antybo genom en näptan fur.

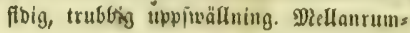
met emellan 8:be orf 9:be nerven på frants wingarne tre gånger fiòre, ån emellan 7:De odj 8:be nerven.)

Cosla Iransversa alarum posticarum 1. inter cosiam bs ot 6 odacte arcuata. 1) Baftwingarnes twermerb fullemligt båg. bòlo emellan 5:te od 6:te wingnerben.

Ramulus costo prima alarum posticarum tumiditato rotundata indictus. Intervallum inter costamalarum anticarum 8 ot 9 vix duplo majus, quam inter costam 7 ot -8 .

Eörfta nerbens på batwingarnes gren, IV. Epinephe antbob genom en runbab uppiwands. ning. Stellanrummet emellan 8:be ods 9:be nerven på frambingarne. fuapt bubbelt ftörre, ãn emellan $\%$ :be och 8:be nerven.

Rnmulus costæe primæ alarum posticarum tumiditate arcuata, acuminata indictus, Intervallum infer I 


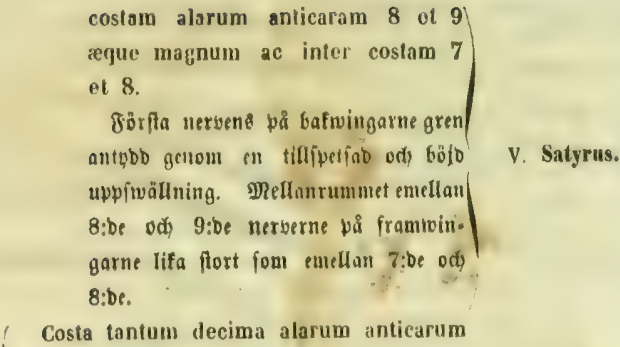

ill. S9lott irammingarnes 10:be nerb wib bas

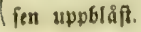

Costa Iransversa cellula discoidalis alarum anticarum inter costam 4 et b arcuata, ad 5 normalis.

Twernerwen wio framwistgarnes biffält VI. Chienobas. emellan 4:be oç 5:te nersen bågböld, wib ben 5:te norntal.

Costa Iransversa cellulae discoidalis alarum auticarum inter costam \& et 5 recta, ad 5 ovanescens.

Imernerben wite framuingarnes biffält VII. Erebia. emellan 4:De ocd 5:te nerwen rät, twio ben 5:te förjuinnande.

\section{Slägtet: Coemonympha. Hüвs, II-S.}

(Hipparchia Fabr. Ochs. Érohia Daly. Satysus Latr. Bolsd, ZetT.) Antenna longiores, exirorsum in Clavam oblongam, subincrassatam sensim transeuntes.

Palpl capite nonihil longioros, subadscondontes, apicibur nutantibus, compressi, triarticulati: articulus medius roliquos 
conjunctos longitudine vix 1. nonilhil superans, aut conformis, aut in modio dilatafus; ultimus basali xqualis, 1. eo multo longior, gracillimus. Articulus medius of basalis infra pilis longissimis divaricatis dense instructi, of in latoribus squamis obtecti; ultimus tamen audus (non squamosus), pilis rarioı ibus, plerumque adpressis, instructus, in pi- lositate reliquorum plus minus latens.

\section{Deull nudi.}

Pedes antisi gracilos, maris brevissimi et densius, fominæ longioros ef parcius pilis ciliati, tarsis inarticulatis. Femora ceterorum infra hirta; tibiæ dentibus brevissimis instructæ, ad insortionem tarsorum calcaribus duobus validis munitæ; articulus tar-orum prinus longissimus (longitudinem secundi tertiique plus minus superans), secundus tertio, hic tamen quarto longior, quintus tortio quartoque longitudine fere æqualis.

Alæ rotundate, integrie; margo interior postiearum ad angulum ani subexeisus. Collula disoidalis clausa.

Costa alarum posticarum 9: 1-4 o trunco communi superiori, 5-7 o trunco communi inferiori, 8-9 e basi egredientes. 1:ma ramulum nullum, interdum tumiditate tamen hamiformi, obliqua, gracili indictum, in lohuw basalem omittons. Costæ alarum anticarum 10 : 1:ma o basi, 2-4 o trunco communi inferiori, $\mathfrak{b}-6$ a costa transversa cellulæ, $7-9$ - trunco communi superiori (7 triramosa ox codom fere loco cum 8 oriens), 10:ma o basi egrediontos. Basis costæe prima, decimæ of llunci communis inferioris valdo inflata. Costa transversa collula discoidalis ramulum rectum breven in cullulam ipsam plerumque omittons ${ }^{*}$ ) ; costa ipsa fortiter arcuata, foro angulata.

* Costa illa valde variat. In C. davo ioterdum simplex, sed interduin etiam ramulos duos gerens. Sis etiom C. arcania, in qua specie proterea ramulum inter costam 4 et 5 , cellula egredientem, observari. 
Untenuerue temligen långa, utåt imåningom öfwergáenbe $i$ en nflăng, sågot tioct flubbra.

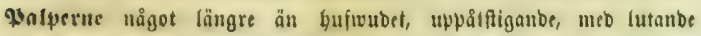

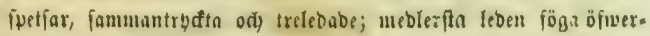
Piganbe $i$ längb be ofriga tílfammantagne, untingen lifformig. eller pó mibten frebare: bell fiffa lifa meb eller of mbefet längre in basleben, ganffa imal. Den meblesfata od) fasleben unbertia

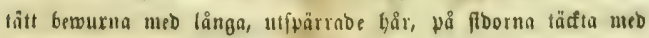
ilälf; den flfa beremot nafen (utnı ffäll) merendels föriedo med gleja, tilltrbata bår, mer eller minbre bolb i be ürrigas hårighet. Sgonen nafna.

Fötterux: främfta paret juala, hod l,amen mydet forta med tätare Gårfranjar, yos gonan beremot längre od) glejare gairfranfabe, sarjerme olednde. De äfriges lår undstill lubne; tibierne före frobe med ganffa forta tänber ods wio taroleben wüprade meb twenne farfa iporrar; tarjernes forfta led längft (ner eller minbre $i$ längd öfwerftigande dell 2bra ods 3:bie); ben 2:bra längre ân ben 3:Die od benı åser längre än ben 4:be, ben 5 :te fill längben näftan lifa méb ben 3:Dje of́, 4:be tilliammans tagne.

233ingarue rumbade, Gelbräbbade; be baftes inbre fant nära anale Görnet แågot uffuren. Difffältet flutet.

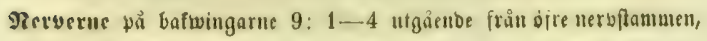
5-7 irån ben nedve, 8-9 irân wingbajen. Den förfă uts fänocr åt wingens rotlob ingen gren, men ftunbom finnes bod ell antypning Dertilf mebeli en bafformig, imed od) jmal upps füälning. Framuingarnes nerver 10: 1: fla utgáende från bajen,

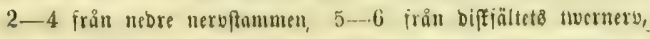
7-9 från öfre neruftammen (beu 7:De tregrenig, uppfommaube nåftan irån camma fălle fom dell 8:be), 10:be trån bafea. Fơr. fta od) tionbe wingnerbent, iant nebre nervfamments rot ulycs

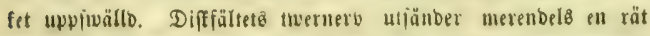
ody fort gren i feelfiwa fältet; netuen ftarft böld, näftan weinflab. 


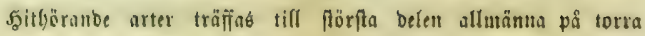

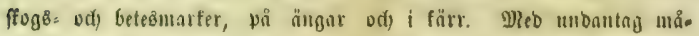
Gänba of blott en art, ibna be jörefomma enbalf $i$ en generation

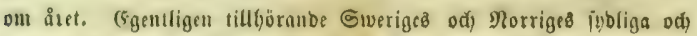

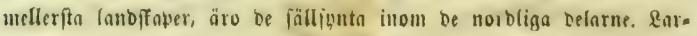
verne äro flảta oç glänfande, utan bår.

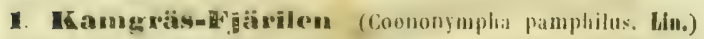

Ilis integris, flavo-octsaresis, anticis utrinģue ocello unico; posticis supra immaculath. subus cinereis, lascia interruptia albida orellisyue 3-4 valdo absolotic. L. al. oxp. 33-36 millim.

פ3ingame beffribdade, ofragula; te trömre med ell ocell på båba fibor; be fatre ofiwn upläabe, undertill med elt aibrutet buit.

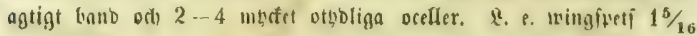
$-1 \% / 16$.

Ş̧n. Papilio pamphilus lín. F. sis. p. 273 n. 1044. Fabr, E. S III. 1. 221. 691. Müller \%ool. Jan. p. 115. 11. 1329. Ilipparehia Aches, selom. 1. 1. p. 30\%. IV. p. 23. 11. 67. Frebia Ialm. P'ap. Sv. p 83. 11. 15. Satyl'us Zett. Ins, Lapp. p. 905 . n. 17. findart m Dupouch. Lepid I. p. 176 pl. 8 socund. r. 3. Coenonympha $\|-\mathbf{S}$. Text I. p. 84. Papilio nephele Ilïbn. P'ap. Lath. 54 f. 237 238. 239 .

Flärilen förefonumer i menue generationer oun äret, ben förfta i M(al oci) Suni, ben anbrn i 2(ugufti or) Eeftember manaber, jamt ar teßumarfer oda göglända ängar unber beuta tib allmän i be föbra

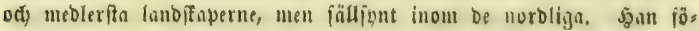
refommer biobe $i$ ffogstratter od på finttlumbet. WBio $60^{\circ}$ norblig

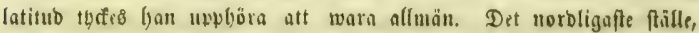
ber ban Morrige, bof torbe yan förefomma ännu norbligare.

2atbeffi. 2(ntennerne bruna med ljujare flubba od butita rill. gar. Balperme Guxitgra, unbertill meb långa grỉ od fiwarta

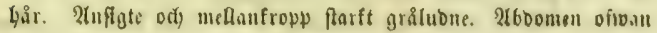
grå, uแbertill buitagtig. Fötterne bwitgrå. 
Samme. WBingarme runbabe, lelbrübbabe meb gråajtiga framfar; ofwan ofragula. Framuingarnę främre fanter och alla wingarnes utfonter frumagtiga. Frammingarne nüra wings fipetien förjiboe med en fiwart, ocellformig, fiörre punft. Fram= tringarne unbertill wib bafen griobruma, i biffen röbgula, i frams. fanten odi) utfonten affegrå; $i$ frambörnet förfebde med en fwart ocell, omgifwen af en blefgul ring ofi probo meb buit pupill. saftwingarne unbertill gråbruma, mot roten mörfnre od belvux= ne meb Grwitgrå lubb; från fränre fanten inhit mibten af tvin= gen går ett lywitagtigt, affrutet banb ofi bafom betta finnab 3-4 merenbels mbdet otabliga, ocellifa bunter.

Syounn, fom är pörre, lifuar Gannen, men har fmalare brutagtiga fanter på wingarnte b̈ fre floa od) liufare utfant på De batres unore fiba.

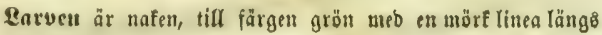
rbggen odh en firwit längs Givarbera fiban. Den lefwer på fiera grágarter, men ifbnnerfjet på famgräfet (Cynosurus cristatus). Pupan är flăt, utan taggar, men bar fantigt lyufwub od är till färgen grön.

2. Starrgrais-1 järilen (Coenonympha davus, Nabr.)

Alis integris, flavo ochraceis, anticis utrinque ocello unico, subtus linoola albida; posticis supra ocelli: $2-3$, subtus cinereis fascia abrupta albida ocolli que $5-6$. L. al. exp. $35-40$ millim.

33ingarme helfräobabe, orfagula, be fräntre på båba fobor meb en ocell, undertifl proboe med en fort, Gwitngtig linea; be fafre ofwan mé 2-3 oceller, unbertill grå meb eft af́brutet quitagtigt band od, 5-6 oceller. \&. e. wingip. $1 \frac{7}{16}-1 \frac{5}{8}$ t.

Syn. Papilio dames Nabr. E. S. III. 1. 221, 690 llipparchia Ochs. Schm. I. 1. p. 302. IV. p. 23, 66. Ereloia Dalm. Pap. Sr. p. 83. n. 14. Satyrus Zett, Ins. Lapp. p. 904. 15. Godart of Duponch. Lepid. II. p. 155. pl. 2 1. I. 4. 2. Cocnonympla II-S. Tost I. p. 8\%. Papilio tullia llübn, Рap, t. 52. f. 243.244. 
23ar. h) Bafwingarme unbertiff Grusa, pubrabe med ofragult; ett lrebt afbrutet fiwitt ('anb öiwer biffen od) wib analfïrmet 2 förre oceller, ontgine af sula ringar odt) förjebbe meb filfuer. färgabe pupiller. (Ale posticar infra fiscie, flavo-pulserulatx, fascia lata, abrupta, albida in diseo, ol ocellis 2 magnis, aryonten-pupillatis, flavediar circumeinetis, ad angulum ani).

Bar. c. Zramuringarne ofwan utan oceller, unbersill meb en; bat. twingarne unbertill med 3 :แe. (Alæ antica supra cooce, subfus uniocellatæ; posticæe subtus ocellis 3 ).

Syn. Papilio laidion Borkh. Sclim. I. p. 91. I, 29.

3) $3 x$. d. Framuingarne oiman bleft ofragula utan ocell, unber. till beremot meb en, hwilfen bod fumbom är otyblig och för. jwinnande; bafningarnes unbre fiba utan eller med en otyblig ocell. (Alie anfice supra fallide ochracese, coece, inIra ocello unico, interdum evanescente; postice coece aut ocello tantun obsoleto).

syn. Papillo isis Thunb. Diss. Ins. Sr. 2, p. 30. Satyrus Zett, lus, Lapp. p. 905. n. 16. Erebla davus rar $b$. Dalm. Paip. Sv. 83. 14. Ochs. Schin, 1. 1. 302. 65. fiodart et Dupouch. Lepid. II. p. I55. Coenonympha isis II-S. Text I. p. 84. tab. 61. Г, 293-296.

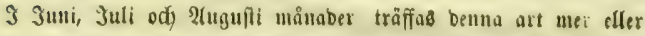
minove salrif $i$ färs ợ pâ fanfa ängar fåmäl $i$ jöbra fon $i$ norra

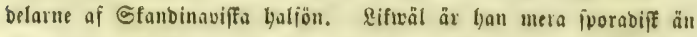

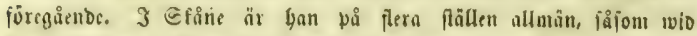

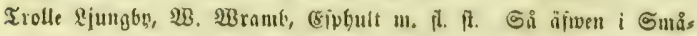

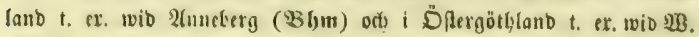

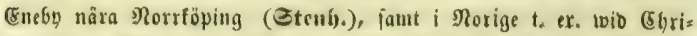
ftiania, (Eoaterg od) i D̈prerbalen (ङicbte). MRot norben nftager lyan bod, ody är fâlfynt inom polcirfeln. De olifa varieteterne föres fomma tillînman meb hraranbra. $\mathfrak{3 a r}$ b., äfwenfom bar. c., är

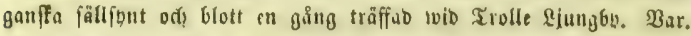
d., fom of några författare blifwit anfedo för egen art, är lifalebes rar oda förefonumer egentligen $i$ norben, ber ben är anutätf wib 


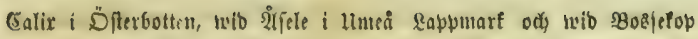

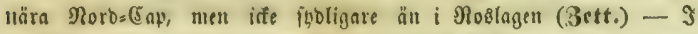

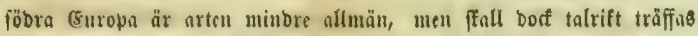
$i$ offra belarne af Eranerlfe.

Irtbeffr. 2ntemmerne Gruna, på ena fiban furitringlabe meb mörf flubba, på ben anbrn beremot enfärgadt grị änba ut $i$ flubbans ipets. ßalperme grångtige med linga, mörfgrå håa unbertifl. 2lnftgtet od) mellanfroppens rbgg gulbrunt=firige. Pröftet ftarft grålubet. 2(6oomen ofman mörf6run, unbertill lywitgul.

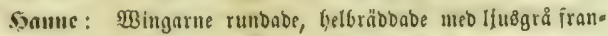
jar; ofwan mer eller minbre mörft offagula, mot utfanterme mörfare; be främre lyafma nära frambörnet en fmart ocellfor= nig punft, omgifiven af en blefgul ring, odf) futubom ännu en anman otybligare punft eller ring; be bafre äro i biffen enfär= gabe mé 2-3 fwarta punfter sära utfanten, htwarbera om: gifne af en gut ring. Stunbom fafnas en eller aunan af besja punfter. Lnbertill äro frambingarne från bafen utöfiver nib. ten ofragula, $i$ utfunten od) frambjomet affegrå meb en firart, breitpupillerab, meb gul ring ongifwen ocell, ody innanför ben= na en twergåenbe bwit linea, fom ef linner någonbera wings fanten; funbom finmes ännu ent minbre on otbblig ocell, oftaft utan pupill. Bafwingarne äro mörft ale egrå meb ett Gyritt, på nibten aforutet fand ö iner biffert, ody $5-6$ med hywita ringat ougifne ocellformiga puntter, oftaft fafunoe pupiller.

Sequan lifnar yamuen till färgen, men är liufare oftragul. Barieteternes olifgeter åro ofwan angifue.

3. Perlgrais-Fjärilen (Coenonympha arcania. Liı)

Alis integris, anticis ferrugineis, infra ocello unico; posticis supra fuscis, infra cinerois ocollis 5 , primo fascia alba continua remoto. L. al. exp. $32-35$ millim.

SBingarme beloräboabe, be fränre roftguln, unbertill meb 1 ocell; De bafre ofwan Gruna, unbertill grångtiga med 5 oceller, Gwaraf beu 
förfta är ffitc från be öfriga genom ett buvitt, fammanlyängande bant. \&. e. wingip. $1 \frac{5}{16}-13 / 8$ t.

Syn. Papilio arcania Lin. F. S. p. 273 n. 104:. Nabr. E. S. III. 1. 221. 692 . Hïbn. Pap. I. 51.1.240. mas. 241. 242. fom. Text. p. 42. n. 75 . Ilipparchia Oclis. Schm. I. 1. p. 317. IV. p. 24. n. T2. Lrehia Malm. 'Pap. Sv. 8\%. 17. Satyrus Godart ot Duponch. Lepil. I. 174. pl. 8. f. 3. Coenonympha II-S Text I. p. 83 .

Denหa art tillgo̊r endaft foggtrafterne, ifbunerlet löfftogarne, $i$

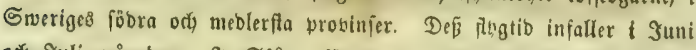
o(j) Juli månaber. $\Im$ Sfåne förefommer lyan nämnbe tib på fera

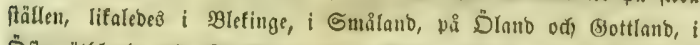

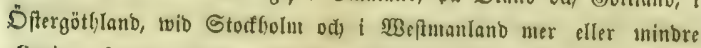
aflmon. $\Im$ norra belarne of lanbet är han änmu iffe anmärft, få att han tydes upplöra meb fifträmmbe provins. Snom Norige är Gan weterligen ị̛e ännu trăffab.

2rtbeffr. 2(ntennerne mörffruma meb brvita ringar. Falperne

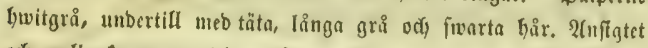
od) melanfropwen gultbruntyåriga. 2660omen ofwan mobrft gulbrun, unjertill fywitagtig. Fötterne gulbruna,

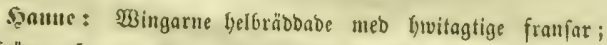
be fränıre ofwon roftgula, länge framfanten fualt, läng utfanten Erebt fwartbruna; be Gafre mörforuna med en fmal roftgul flåd trib analförnet. Jrammingarne umbertill roftgula, i fräurre o(d) thttre fanten gråtruna, meb ett jwartagtigt, ftumbom fifwer= färgabt ftref längo ben feonare. Bafivingarme från roten ulö $\hat{F}=$

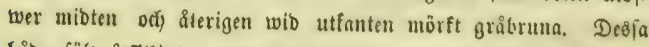
båba fält åtfillias genom ett lywitt Ganb of olifa brebb, four innanför iี่ lyar $i$ wingens fråmre fant en ocell, och utanför

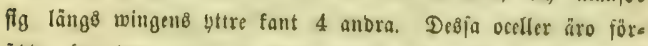
leobe Gwatbera med en lywit pupill och omgifne af en mürt ody en gul ring. Stunbom äro 1-2 af bem otholige; äfwen anthbas be ftunbou ph iwingens öfre fiba. Emellan ocellerne ody gttre fanten löper ett filfwerfärgabt ody ett roftgult fred? 
Sounan lifnar Gannen, men har ofwan i framtwingarnes mörfa utfant en fwars, ai cul linea omgifwen puntt, fom mot. fwarar unbre fibans ocell. Inuanför ben febnare på unbre fiban en otgolig kwit twerfhäd.

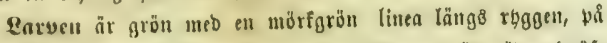

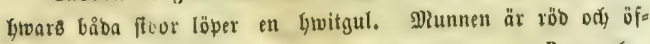
wer fotraben går på lywarbera fiban en gul linea. Pupan far röbagtig aboomen od hywitagtiga wingfibor med röb infattning.

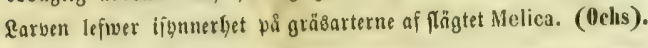

\section{Bruna Grajs-Bijarilen (Coenonympha hero Lín)}

Alis integris, luscis: anticis supra ant immaculatis, aut ocello minutissimo, infra distinctioro; posticis supra ocellis $2-5$ coecis, infra 6 pupillatis fasiaque dentata alba. L. al. exp. 30-32 millim.

SBingarme belbräbbabe, fwartbrutha, be främre ofwan antingen ofläafabe eller förfebde wed en ganfa liten ocell, fom unbertill är ftorre ody tholigare; be Gafe ofman meb $2-5$ blinba, unbertif med

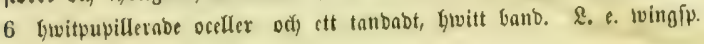
$11 / 4-15 / 16$ t.

Syn. Papilio hero Lin. F. Sv. 274 1047. Itîlon. Pap. t. 53 ศ. 2:i2. 253. tem. Texl p. 42. n. 76. llipparchia Ochs. Schm. 1. 1. 333. IV. 23. 70. Erebia Dalm. lap. sv. 84. 16. Satyrus Zett. Ins, Iapp. 905. 18. Godart et Dupanch. lepid I. 172 pl. 8 secund. f. 2. Coenonympha Ii - S. Text I. p. 85. Papilio sabous fabr. E. S. III. 1. 222. 694 .

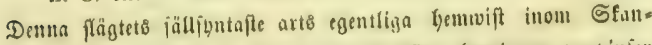
binaten är Eweriges ode Shoriges mederfta, bergigare provinjer emeflan 58-62 graber norblig volf̧ölo, ber han uiubre fälan trä

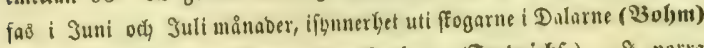

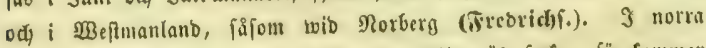

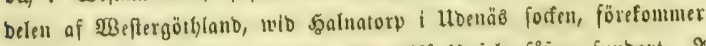

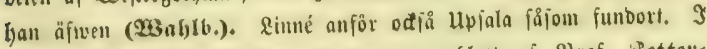
Gfane är ban bügft fäljint ody ber tagen blott af \$rof. Setter:

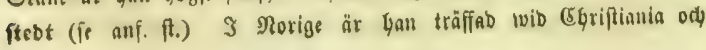


Songsiwinger af Doctor Bicbfe. Tnom be egentriga \&appmarfernc är ban ef anmärft. Utom Smerige är ban funnen på åtfiflliga ftällen i Tyftlano odi Norbfrantrife.

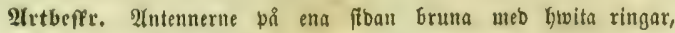
pณ̊ ben nnbra enjärgabt grå. Balperne på längben lywita oḍ

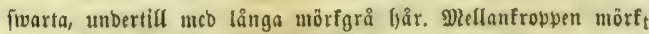
brungdrig. 2l6oomen ofwall mörfbrun, unbertill gulfyit. Föt= terne brungrå.

Saante: wingarne belfräbbade med Guvitagtiga franfar; ofwan mörffruna, be främre oftaft enfärgabe, fällan med en ocellformig fläc i trambörnet, beffående nf en mört puntt, foum omgifwes of en matt, gulagtig ring; De bafre nära utfanten meb 2-5 ocellformiga fäfour, oftaft utan pupiller, omgifne fwarbera med en gul ring; i analförnet ctt fmalt röbgult ftreff. Unbertill äro wingarne vofrafruna; be främre ofta meb en liten Lywitpupillerab, af gul ring omgifwen ocell; innanför Denna fones mer efler minbre tholigt en fort, bwit linea. Bafo wingarne bafwa bafom mibten ett twergående, merenbels fams manţänganbe, tanbabt, fowitt banb, utanför hwilfet funnas 6 fiwarta, Gwitpupillerabe oceller, fom huarbeta äro omgifue of en röbgul ring. \&änga alla wingurnes b̧ttre fauter löpa en Glḅe färgab, od en röbgul linea.

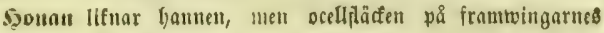

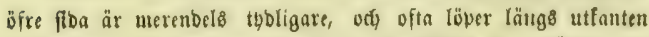
af bafuringarnes öfre fiba ett fmalt, röbgult fireff. 2̈ fwen är

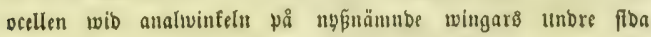
ofta bubbel.

21แm. Til Gweriges Fonuna bar äfwen blifuvit räfnab Darr.

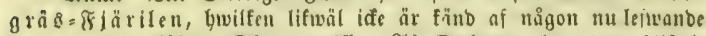
Repibopterolog fafom Sfundinuviff. Af Dalnton har ben blifrit upvförd på grund af ctt till (6i)llenbal jändt exemplar utan locals uppgift. För widare sfterforftuing upyförcs bär beß̧ artuärfe:

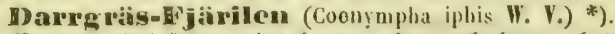

WBingarne lyelfräbdabe, Los ganmen ofwan oftabruma, fyos bos

") Intra limites Scandinavim non occuril, quantum novimus. 
nant offragula; be bafre umbertill brunagtigt grå meb eft afbrutet hivitt banb, 5-6 ocrller ody en röbgul linea bafour en annan fmal, filfwerfärgad i utfanten.

Syn. Papilio iphis V. V. Anh. p. 321. 2\%. Miilon. Pap. t. 53. 5. 249 mas. 250. 251 fom. Text p. 40. 71. Ilipparchia Ochs. Schim. I. 1. 310. Satyrus. Godart ot Duponch. Lepid. II. P. 145. pl. 20. f. 3. 4. Erebia Dalm Pap. Sr. 8i. 18. Coenonympha II-S. Text. p. 85.

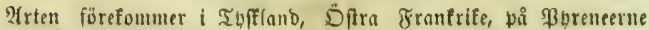

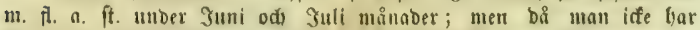
ftg befant niggon fäter local för beß̉ förefomf inom Sfanbinavien, tan ben et ännu räfnå̊ tiff beß̉ gauna.

\section{Slägtet: Darargà. Hüвn, H-S.}

(Ilipparchia Farr. Ocis. Satyrus Latr. Borsd. Zetr. Erobia DaLm.)

Antenמa longiores, extrorsum in clavam distinctiorom, plerumque sat doterminatam, ovatum, sensim transeuntes.

Palpi longitudinem capitis vix I. parum superantes, subadscendontes, apicibus nutantibus, compressi, friarticulati. Arliculus medius religui conjunctis multo longgior, ad basin basali latifudine equalie, exinde sensim allonuafus, apico rotundato; ullimus minulus, basali multo brevior, ovaflls, ad hasin medio gracilior, ad apicem tamen illo laliludino foro xequalis, rotundatus. Articulus medius, ut etiam basalis, infra pilis longissimis pareius instructus; ultimus pilis rariorihus ohsitus. in pilositate reliquorum fore occultus.

\section{Oculi hirti.}

Pedes antini gracilos, maris brovos ot densius, feminæ tamen longiores of parcius pilis ciliati, tarsibus in maro inarticulatis, sod in fomina articulis $3-5$, debilibus. Femora ot tibite coterorum fore ut in Coenonympha; sic otiam articuli tarsorum. 
Alæ rotundatæ, subdentatæ, margine interiori postiearum ad angulum ani rolundato. Collula discoidalis anticarum subaporta.

Costx alarum ut in Coenonfmpha, sod ramulus costæ primæ posticarum tamiditato subquadrangulari, ad finom soepo latiori, indictus, ot basis costæ 10:mæ ot trunci communis inferioris tantum inflata. Costa transversa cellulie discoidalis ramulum rectum et brovem in collulam ipsam somper emittit; çosta ipsa non tam fortiter arcuata, ut in Coenonympha, ot inter costun 4 et 5 plerumque abrupta, tantum plica tenuissima continuata.

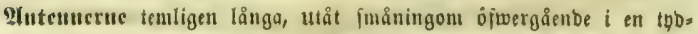
ligare, merentels temligen beftämb od) äggformig flubba.

भalperue fnapt eller bögft obetbbligt b̈werftigande bufwubets längb, upyåtfliganbe, neb lutande fpelfar, fammantrufta off trelebade: meblerfta leben mb̨đet längre än be öfrige tillamumantagne, wib bafen i brebo lifa bå̊leben, berefter fmåningon affmalnande, $i$ fpetjen tillrunbab; ben fifta liten, miłfet forture än basleben, äggformig, wib bajen fmalare än meblerita leben, men rrib ịpete fen $i$ brebd näftan lif benjanmma, afrunbab. Mieblerifa leben temte başleben undertilf förfedo meb glefure längre här; fiffa leben föga lyårig, $i$ be öiriges̊ bårighet näflan bolb.

SBoueu lubna.

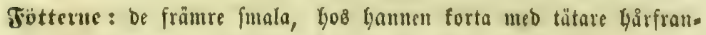
far, bos Gonan beremot längre, ody glefare gårfranjabe; tibierne fos Gannen olebabe, hos tonan belabe i $3-5$ rubimentera les ber. De ofrige füterne lå och tibier, äjwenjom tarsleber lifa Dem Łos Coononympha.

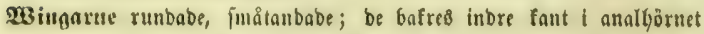
runbad. De frâmrę biffätt Galföpyet.

Merverue på wingarme fâfom hoo Coenonsmpha, men förfta wing= nerwens gren pî bafwingarne angifwe genom ell näftan quas brüngulär uppfwällning, jom ofta är brebaft mot flutet, odf blott 10.be wingnervens faut nebre neruptamuens rot är upp=

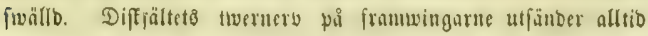




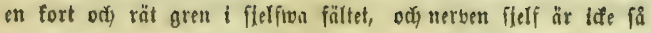
ftarft bölo fom hoo Coononympha od) merenbels afbruten uels lan 4:be och 5:te wingnerverne, od blott fortfatt mebelft ett fint wingrved.

De till bettu fägte räfnabe arter, flwilfa förebraga fteniga,

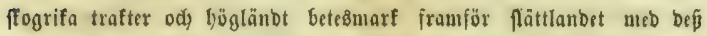
åfrar, tillyöra, meb unbantag af en enba, föbra odj meblerfía Sives rige ory Rorige. Störfta belen förefonma of i twenne genationer our året, 'ben ena, Yywilten öfwertsintrat, on wiren ody ben anbra um böften. Sarberne äro lubna af fina ody forta baar.

A. Antennklubban langsträckt, föga afskild fran skuftet.

1. Dảrgris-is-järilen (Pararga dejanira Lin.)

Alis subdentatis, cinereo-fuscis; anticis utrinque ocollis 5, magnis: posticis supra ocellis 2-4, infra 6, fasciaque albida. L. al. exp. 49-57 millim.

THingarme jmâtandabe, gråbruna, be frămre på båba flobor meb 5 , be fafte ofivan med $2-4$, unbertill med 6 occlet od eft Lywitt tmerband. \&. e. wingip. $2-2^{5} / 16$ t.

Syn. Papilio dejanira Uín. S. N. 1. 2. 774. 154. Fabr. E. S. III. 1. 230, 719. lliilon. Pap. t. 38 f. 170. 17 f fem. Toxt. p. 29. 29. Ilipparehia Ochs. Schm, 1. 1. p. 229. IV. p. 21. 29. Satyrus Godart of Dupouch Lepid. I. p. 168. pl. 8 f. 1. Pararga II-S. Toxt I. p. 87.

Demna, meblerfta (surupa sgentigen tillböriga, art, guxiffen iīgn=

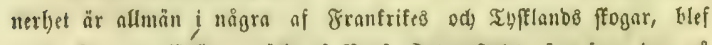
inom Swerige förft upptäft af ßrof. Betterfteot, foum faun ben på

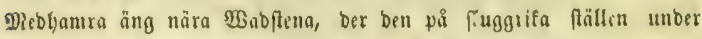

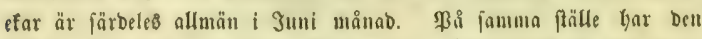

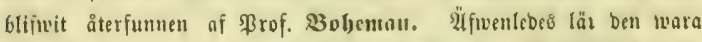
träffa wio Drotmingholm af Stub. Belfrage. 2(nnorftäbes inom lanbet är ben ef numärft.

21tbeffr. Antennerne Eruna, buitringlabe. Palperme Givita, neotia fwarta. 2Infigtet juartagtigt, Grunlubet. D̈gomen bafåt 
begränfabe af ett butitt flred. Mellanfroppen gråagtigt brums luben. $\mathfrak{a b o m e n ~ o f w a n ~ f r u n g r a ̊ , ~ u n b e r t i l l ~ l u r i t a g t i g . ~ B r o ̈ f t e t ~}$ gråluoet. Fötterne ḩwitgrå.

Sุanuc: Wingarme fmåtunbabe meb fuvita, på be fråmre fwartflåcfiga franjar; of̣an gråagtigt bruma; be främre nted 5 , be bafre med $2-4$ ftörre od minbre fwarta, ocellifa fiädar, bwarbera umgifue of en gul ring. WSingarmes unore fiba llu. inte grå meb en fywitagtig, apang fläa $i$ lywarbera mingens bifffâlt, ody ett winflabt, \{ysitt, pai framtwingarne i gult flötanbe, Hå Gafmingarme uäfan affrutet twerbano närmare utfanterne, bafom busilfet finnas på be förra 5 ftora oceller, fom äro näs fan lifa öfre fibans, men bafwa lyarbera en Knvit pupill, oç pai be febuare 5-6 oceller, af Gwilfa be 2:ne, framtanten när. mape, äro ftilda från be öfriga genon bet liwita banbet, fom

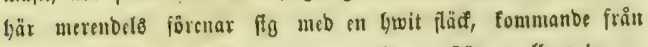

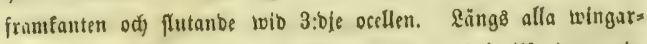
nes utfanter löpa tremue mörfbruna linier, af Grsilfa ben mebs lexfa är Grebaft oct tholigaft.

Şounu lifuar lyaumen, men bar bet frwitugtiga Ganbet på wingarnes unbre fid me merendels fmalare ody fafnar oftaft ben brvita flăfen fiafom ocellerme tå bafuingarne, eller har ben blott matt antopb.

Sarven är finluben, ljuฮిgrön meb mörfare linier längs

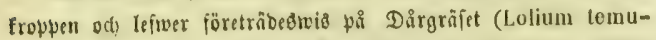
lontum), el)uru ben, fåfom fina öfrige famplägtingar, äfwen tillgriper anbra grãg̊arter.

\section{A. Antenklubbun äggformig, tydligt afskild frin skaftet.}

2. Sivingelgrais-Firilen (Pararga megxra. lin.)

Alis subdentatis lulvis fusco-lasciatis; anticis supra ocello, posticis supra ocollis $3-5$, inlra 6. L. al. oxp. 45- 19 millim.

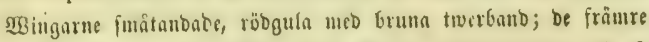
ofiwan med en ocell; be bafre meb $3-5$ oceller ofwan, od 6 unbertill. \&. c. wingfp. $1^{19} / 10-2$ t. 
Syn. Papilio megcera Lin, S, N. 1. 2. 771. 142. Vahr. E. S. III. 1. 94. 222. Ilübn. 1. 39. I. 177. 178 mas. Toxt. p, 29. 31. Ilipparchia Ochs. Schm. 1. 1. 235. 1Y. 21. 33. Treitseh. X. 1. 37. 226. Erebia Dalm. Pap. Sv. 79. 3. Satyrus fodart of Duponch. Lepid I. 160. jI. 7. sext. f. 3 fom. Pararga II-S. Toxt. I. p. 82.

Uti ibbligafte provinisrne ni Giverige är betna ftäril om wåren i Mlaj od) feban siter $i$ Juli, men if̧unerfyct $i$ Yugufti månab iffe

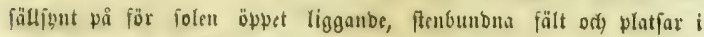

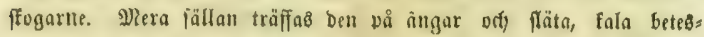

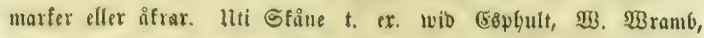
Trolle \&ungby m. ז. ft. od) i Blefinge t. ex. wio Gölwestorg od (5arlabamn är ben temligen allmản. I Smålanb (Mrioberb.), ร̧al=

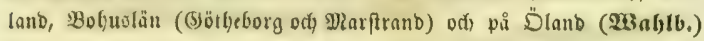

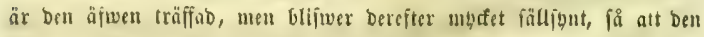
blott en eller altuan gång $i$ Juni månad förefommer wib Shriftin. nia $i$ Norige. Således funes den fiti.ja bögre mot norben wâ tweftra ån üftra fuften, emedan ben iffe blifivit anmärft i D̈ftergöthlano

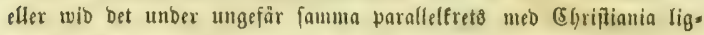
ganbe Norberg i Deptuanlanb, iffe eller tvib Stodholm. Mlyfet gögre än till (5lyriptiania torbe ben bod iffe eller 1 twefter gå.

Mrtbeff:. 2(ntemerne mört6runa med lywita ringar. Stubban mörffran, på ena fiban buvitgrå. Balperne gråagtige, meb långa mörfgrå od) brunagtige bair unbertill. 2nffgtet ods melanfropden lubne of gulbrunt. 266omen of wan mörfbrun, unbertia gråagtig. Bröftet mörft grålubet. Fötterne bruna.

sanure. WBingarne fmåtanbabe, ofwan röbgula, wib bajen mörfare, lisnnerlyet de bafre, i framfanten odj utfanten mörfe bruna, meb gråagtiga franfar. De främre med eft mörforunt ttucrband öfwer biffen, lywilfet gåx från framfanten, läng biff= fältet及 twernerv, wid Girars flut bet delar fig, fänoande en fmnlare gren till twingenछి bafre hörn, ber bet innefluter en fläd of grunbfärgen, od) en brebare till infantenti mibt. Defisutom finnaB twenne forta ody fmala mörfbruma linier $\mathrm{i}$ bifffältet oda en fwart, jowitspupitherad ocell $t$ främre Görnet. Bafwingarne, 
fwars röbgula färg fmarare bilbar ett twerbanb, har wib ut, fanten 3-5 olifflora iwartn oceller, fuvarbera meb en lywit pus. pill. Ulıbertill äro framuvingarme blefare än på öfre floan odf) i minglpetfe. grångtige, jamt bafwa längo ufanten blefa parals lela linier eller fäcfar. För öfrigt äro be tecfnabe fåfom på bire fiban, men twerbandet fafnar ben inbre brebare grenen, od ocellen, brwilfen frunoom framom ffg far en minore, är omgifs wen of en brumagtig ring, fom innefluter en amman röogul. Bafuingarne äro unbertill grå, mot fafm mörfare och bafwa ofwer biffen tmenne tanbabe fruna linier, jom innefluta ett twerteand af grunbfärgen, ofly längs utfanten 6 fmärre oceller, Grarbera omgifne af twemue bruna ody trenene röba ringar. Den inbre ocellen är oftaft bubbel. Sängo ben mörfare utfans

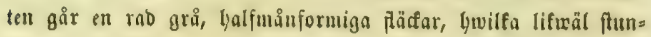
bom åro otubliga.

Syounn är fërre än banneH med blefare grumbiärg, od twerbaubet på frammingarnes öfre fiba fafnar belt od hâflet

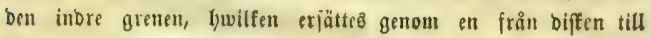
inbre fanten gåenbe linea.

garven är grön med 3 :He mörfa linier, en länģ ruggen B(h) en längs fwarbera froppftoan, od) lefwer på åtfilliga gräß. arter, if̧muerbst på fivingelgräjet (Festuca elatior). - \$ups pan, fom är grå eller fwartagtig med ljujare fmå taggar, bar twenne trubbiga ipetiar på hujwubet ody en trubbig tagg på ruggen.

\section{Berggräis-Fjä rilen ('’ararga hiera llübu.)}

Alis subdentatis, fuscis; anticis utrinque sesquiocollo; posticis supra linea transversa nigra, dentata, ocellisquo 3, infra 6. L. al. exp. 43-45 millim.

W3inz̧aเne futåtanbabe, Gruna; be främre på båba fibor meb en ftörre od) astubning till en minbre ocell; be fafre ofwan meb en twergåenbe fwart, tanbab linea od) 3 oceller, unbertill med 6 bplifa ฉ. e. wing $\left[\right.$ p. $1^{8} / 4-1^{18 / 16}$ t. 
Syn. Papilio hiera Ilïbm. Pnp. t. 39. f. 176. fem. llipparchia Ochs. Sclsm. IV. 135. Treitsch. X. 1. 36. Satyrus Godart et Duponch. Lepid. Suppl. I. 286. pl. 46. f. 3-4. Boist. Icon. p. 220. pl. 44. f. 1. 3. Pararga II-S. Texl I. P. 89. Satyrus mara var Godart. Eneycl. mothod. tom. IX. 501. 86. Moisd. Ind. motb. 21.

Denna art tillbör mera Sfanbinaviffa lalföns meblerfta od norra lanbftaper, än Defi föbra. Dod färefommer ban äfwen, elyuru

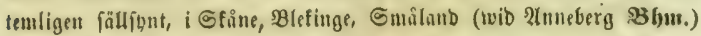

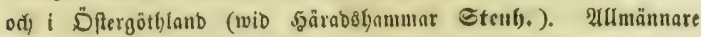

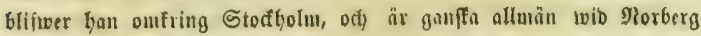
(Frestid).) i Weptmanland. I föbra Rorige funes ben if์e fäl.

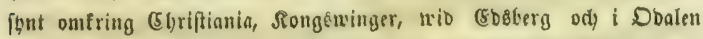
(eicbfe). 3 be norbligafte lanbftaperme träfias ben offwer all i

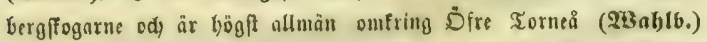
Bergiga och fenrifa ftogtrafter ẩ beß̉ egentlig tillbål od flogtioen infaller i Maj, Juni od Juli månaber.

Urtbefr. Antennerne fwarta meb bivita ringar. STubban fwarts Grun, på ena fiban Gwitpubrab. Balperne brungrå, unbertill

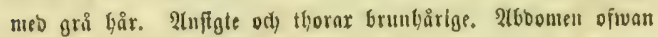
brun, unbertia gråagtig. Bgröftet brungråtf, lubet. Eötterne bruna.

Sanue: NBingarne runbabe, meb bruna fronfar; ofwan bruma; de fränure neb othbliga mörfare linier $i$ biffen od en rab röggula fäcofar $i$ frambörnet od) wio utfnnten. 3 främre börnet finnes uti en röbgul flä en ftörre iwart ocellformig flä med buvit punft i mibten och en amman bulif wio. fanten; flunbom finnes en Gyvit, mco frwat ongifwen, sunft nărmare mingipetion. Bafwingarne l,afwa öjer biffen en frwart, eller mörfbrun, tanbad linea odt) längs utfanten en rab of 3:me ftöre oceller, ymartera omgifue af en röbgul ring orif för= feboba meb lysit pupill, famt uråt begränfabe of en fill mơrf: trun linea. Gtunbom finnes äfuen antyoning till on eller allo nan ninbre ocell. Undertill äro framtvingarne fotigt fruna

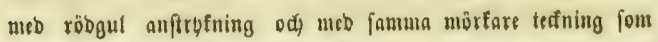


megæra, famt bafma i frimre bornet en ftor, af röbgul ring ougifwen ocell, fyuilfen ofta yar twenne minbre framom fig i wingfpetfen. Bafwingarne äro unbertill grii, mot bafen mört* Gruna; föröjrigt tıll teffniıg od) oceller lifa bem yos megera.

Sonan lifnar bannen, men fjar ljufare granbjärg, odg tpo. ligare tedning ofman.

\section{Idvitgris-ginilen (Pararga mæra Lin.)}

Alis subdentatis, obscure fuscis; utrinque anticis susquiocollo; posticis supra unicoloribus ocolliss 3 , infra 6. L. al. oxp. 49-55 millim.

W3itgarne fmaitnobabe, mörft bruta, be fränıre ofwan méb en fiorre od) antbbaing till en minbre ocell; be bafre ofwan enfärpabe meb 3 oceller, undertifl mid 6 . \&. e. twingfp. $2-2 \frac{1}{4}$ t.

Syn. Papilio mera Lin. F. Sv. p. 275. 1049. Fabr. E. S. III. 1. 227. 711. llïbn. Pap. 1. 39. ґ. 174. 175. Ilipparchia Oehs. Schm. I. 1. 231. IV. 21. Treitsch. X. 1. 36. Erebia Ilalm. P. Sv. 79, 2. Satyrus Zett. Ins. Lapp. 901. 1. Pararga II-S. Text I. p. 88. Satyrus adras/us Dupoucl. Lopid. Suppl. I. 284. pl. 46. f. 4.2.

21um. Satyrus mara Godart Lepid. I. p. 157. pl. 7. soxt f. 2. är famma art fom llipparchia adrasta (Esp. Ochs. Schm. 1V. 21. 137.) od - iffe Limes P. mara, Dod åt ben mágända blott en varietet af benna febuare.

Riffom füregnenbe art egentligen tillbör be norra trafterne of

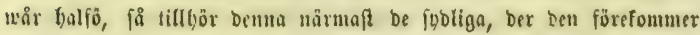

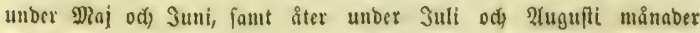
mer eller minbre allmän uti ftogstrafterne, på fält od, ängar. Den f̧neถ $i$ măngo aftuga mot notben $i$ famma man fom föregåenbe till. tager, men buru langt ben giir $i$ norr är lifnäl änuแ iffe utrönt. Wib Storfboim ody $\mathrm{i}$ Beftmanland fimures bell ods Brof. Betterfitebt uppför ben fäfom fälfsht i Rappmarfens fogar. I Morige före= fommer bell åtminftone änba till Tronbljem (Sicbfe).

Artbeffr. Demma lifnar få nära föregåenbe art, att flera älbre Fonjattare anfett bem båba faijom variationer of ell of famma. ફูär är tîlxäđfligt att angifwa frilfaftigheterne: 


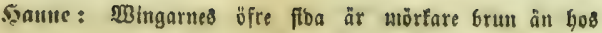
1. hiera, utan alla fpår till ben mörfare teçning, fom finnes yos nดฉ̄nämnde. Zramwingarne $i$ frảnte Görnet tilfpetfade meb en fiörse, af en jual röogul ring omgirmen ocell, od framför benna en minbre, lifalebez omgifwen af en röbgul ring. sonfwingarne hafwa ocellerne fäjom bos $P$. hiera, ven utan

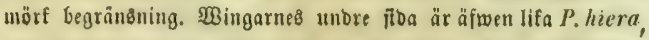
men framtringarnes ocell bar oitaft ännu en hwit lăd bafon Den wanliga pupillen.

Sgonat, fout far frantwingarne mera runbabe än Gamem, är till färgen ljụare, än benne, men äfwenledes utan mo̊rfare teffning od) har ofta fåfom $P$. hiera, några röbgula fläfar $i$ framuingarnes yttre fant, bafom ocellen, Grwilfa hannen till benna art merendels jafuar.

Earven år ljuళggrön med on mörf rbgglinea od Gwita frimmor lings froppsfitoorne famt bewuren meb fina, byita bår. Den lefiver på Hlordeum murinum, Poa annua, Festuca fuitans m. ก. gräвarter. \$uppan är trubbig, fwarts agtig eller blefgron. (Ochs.)

\section{Auiclkgrais-Fjairilen (Pararga egeria Lin.)}

Alis dentatis, olivaceo fuscis, Juteo maculatis; utrinque anticis ocello; posticis supra ocollis 3 , subtus punctis $4-5$ albis, fusco-circumeinctis. L. al. exp. 42-46 millim.

Wingarne tanbabe, olibstruna, gulpläffiga; be främre på bảba fibor med 1 ocell; be bafre ofwnn med 3 oceller, unbertill med 4-5

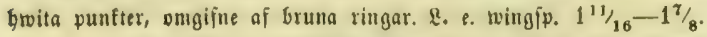
Syn. Papilio egeria lin. S. N. I. 2. 771. 143. Mabr. E. S. III. 1. 94. 293. Ilübn. Pap. t. 40. f. 181. 182 mas. 'Text. p. $30^{-}$n. 33 . Ilipparchia Orhs. Schm. I. 1. 238. IV. 21. Treitsch. X. 1. 37. Erebia Ialm, Pap. Sv. 79. 1. Satyrus fiodart et Inponeh. Lepid 1. 163. pl. 8. secund. f. 1. Pararga H-S. Text: 1. p. 89.

Denne färil, en of wår Fauma fällientafte arter, är, fânibt man wet, Inom Smerige endaft funnen $i$ be mederfin lanbffaperne, fåfom på Lanbtbrufo̊ndabemienó cxperimentalfălt wio Stoctlolm 


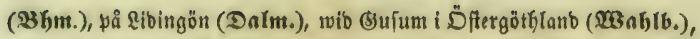
wib Borgholm på D̈land (3ett.) famt twid Sapellehamn pi̊ (5ott= Yand, ber tag träfifat Den fommaren 1849. I Rorige förefommer bent

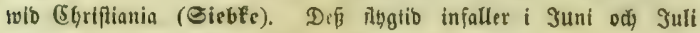
månaber, b\&̊ arten ifynnerlyet bör föfas $i$ barrffoga:.

21'tbeffr. 2(ntennerue ofwan Gruwa med Yuita Iebafbelningar, unbertill buvitagtige meb röbgul anfitęfning och brum lebafbels ningar. Silubtan brunagtig meb rảbgul pets. Balperne grås gula, meb brumagtige gåt unbertifl. Mellanfropp od, abbomen ofwan bruna, ben före luben. 2t6bomen unbertill gråatig, åfเeenfom bet lubua bröftet.

Syanue: WBingarne, ifpnnerfet be Gafre, tanbobe; be främre meb omfring 12 gula flådar, od ell fwart, lywitpupil= lerad ocell, fom utåt begrân fas af en gul bolfcirfel; be bafre meo 2:ne otyoliga gula fäafar, bels ena i framfanten, ben anora emeflan 4 ody 5 wingnerberne, od läng uttanten $3:$ the förre fiwarta, Emitpupillerabe oceller, bwarbera omgifne af en gul ring. I rab meb beşa finnes en gư fläaf, fom àr förfebd med en brun bunft. Ilnbertill äro framtwingarne blefare ody $i$ fpetien gulbruna, men für örrigt tectnabe fâfon på öfre ftban. Bafruingarne äro ncbantill gulbruna, längg utfanten ftotunbe $i$ grått ech lizunbom der föricoba med en nörfare linea. Dofuer biffen gå turenne tandibe, mer eller minbre tụbliga bruna linier, mellan bn ilfa grunbfärgen ftunbom är mörfare ody ba bilbar ett twerbano. Balour Detta funas twenne gulattige fläcfar, stot= fwaranbe offe fobans. Rängs utfanten finnes sn rab lowita punfter (4-5), Gwarbera omgifna of en mo̊rf, röbbrun ring. Eranfarme oftwan ody unbertifl gulagtige meb brutn päffar ber nerverne utlöpa.

Sgouan lifnar bannen, men bar $i$ aflmänbet blefare färzer.

Larven äน Gุårig, pả tweren fruffg, mattgrön, längz rดg= gen mơrfgrön, me’o gulagtiga eller butagtiga linicr länģ

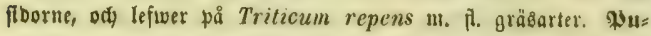
pau är gronn eller brunagtig, med twenne forta od trubbiga 
taggar i Gufwubets främte bel, upvitwällo rygg orh twenne raber juเ fnappformiga utwerter länga ât benfaแmแa.

\section{Sligtet : phantopus. nov. gen.}

(Ilipparchia Fabr. Ocns. Salyrus Iatr. Boiss, Zktт. Erebia Dals. Epinephele. Hüвn. H-S.)

Antenuse longiores, extrorsum haud crassioros. Clava indistincta.

Palpi Iongitudinem capitis vix scporantes, subporrocti, compressi, triarticulati. Arliculus medius reliquos conjunctos Iongitudine superans, basali foro latior, sed conformis, ad apicem haud altenuatus, apice subtruncato; ultimus basali longior, omnium gracillimus, cylindricus, apice acuminato. Articulus modius et basalis infra pilis lungissimis, divaricalis dense instructus; ultius tamen pilis adprossis, in pilositate reliquorum non latens.

\section{Deuli nudi.}

Pedes antici brevissimi, in pilosilate plano occulti, pilosissimi, incrassati ; femora sat crassa ; tibix tarsique otiam incrassati; lii arcuato-roflexi, biarticulati, articulo ultimo minuto, conico. Fomora pedum roliquorum infra ad basin hirsuta. Calcaria tibiarum minuta. Articuli tarsorum foro ut in genere Coenonp̧mpha.

Alæ rolundat $x$, integræ. Margo intorior posticarum ad angulum ani.haud excisus. Collula discoidalis anticarum subaperta.

Costa alarum ut in genote procodento, sod costa transversa anticarum semper simplox, inter costam \& ot 5 , laic tamon propius, abrupta, tantum tumiditato continuala, ot inter costam 5 et 6 oblique subarcuata. Intervallum basale inter coslas alarum anticarum 8 ot 9 triplo majus, quam inter costam 7 et 8 . Ramulus costx prime alarum posticarum tumiditato subquadrangulari, oblusa, indictus.

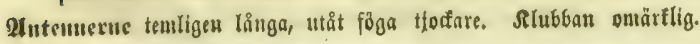


Warperte funppt bf fwerfitiganbe hufwubets längb, framårigigtabe, fauts mantrbatte, trelebabe. Mederfta leden längre än be öfrige tills fammantagme, näftan brebare än Eagleben, men för öfrigt lif= formig, wib fpetien föga nffmalnanbe, trubbig; ifta leben längre ăl baslebell, fmalare än någonbera of be öfrige, colinbrift, till. fpetiab. Den meblerfta leben temte basleben unbertill föriebo meb långa, utåtjperrabe, täta hår ; ben fifte beremot neb tials tryatia bair, ide bolo I be öfriges bårighet.

\section{Sgonen nafna.}

Fötterue : be främtre bügft forta, allbeles bolba $i$ bröftets bårighet,

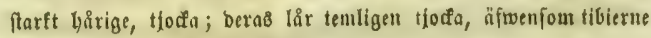
od) tarjerme, quwilfa febnare äro bågböjot bafåtrigtabe, twålebabe, atterfta leden liten ody conift. De D̈frige fötternes lår unber: til wib bafen lubna. Tarjernę fporrar futå. Taralederne näs fan fom 408 flägtet Coononymplia.

23ingarue runbabe, helgräbobde. De Gatres inbre fant wib anal. görnet föga utffuren. De fräures bifffält balföppet.

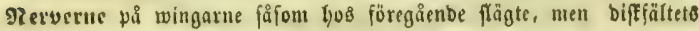
twernerv pó framuingarne alltib enfel, emellan $4-5$ wingnero ben, närmare ben febnare, afbruten, bloft fortatt genom en uppiwillning, och emellan $5-6$ wingnerben fnebt bightöfo. 8-9 uerverne på frambingarne wio bafen filba frân fuxarall. bra genom ett tre gånger ftörre mellanrum äl $7-8$ vingner= werne. Brrenen till bafwingarneb förfta nerv antşob genom en nåftลn quลbrangulär, trubbig ułpfwållning.

Snom Sfanbinabicn förefommer en enda art:

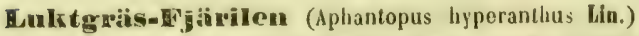

Alis integris, fuscis, subtus anticis ocollis $1-3$, posticis 5 I. Jantum punctis albis. L. al. exp. $35-38$ millisu.

23ingarne lelbräbonbe, bruna unbertifl med $1-3$ ocefler bå frantwingarme od) 5 på Gafivingarme elles blott burita punfter. $\&$. \&. wingip. $1 \frac{\gamma}{16}-1 \% 16$.

syn. Papilio hyperanthus Lin. F. Sv. 2731043. Vabr. E. S. III. 1. 216 . Ilipparchia Oclss. Schum. I. 1. 
225. IV. 21. 28. Erebia Malm. Aop. Sv. 81. 8. Satyrus Zett. I!:s. Lapp. 903. 8. Godart of Duponch. Lopid. I. 170. pI. 7. I. 3. Epinephele II-S. Toxt I. p. 81. Papilio polymeda l'úbro. Pap, t. 3९, f. 172. 173. T'ext. p. 28. n. 28.

Bar. a: BBtrgarne ofwan mòtoruna, merenbels ofläcfabe; un= bertill glänjanbe gulgrå; be främre meb $1-3$ af gula ringar omgifue oceller; be bafre med 5 på famma fätt prbbobe oceller (P. hyperanthus Auct.)

Bax. b. Wingarne ofwan gråbruna, ofläfabe, unbertill glänjanbe gulgrå, be främıre med 2, be bafre med 5 oceller. (P. vidua Auct.)

War. c. Wingarme på båba floor gråbruna, unbertill be främre med 2, be bufte neb 5 buita punfter. (P. arele 0ehs. 1. 1. 228.)

Bar. d. Rif forregånbe, men har ofwan på baftwingarne wib analhörnet twenne Gwita punfter.

Fin̈rilen, fom förefommer unber alla fommarmanaberne, är alls

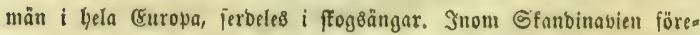

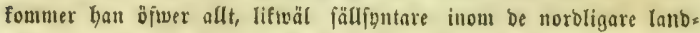

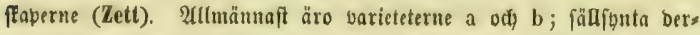

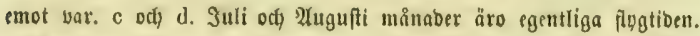

Artbeffr. 2(ntennerne fwartbruna meb hwitagtiga ringar ofi) ljuôbruna fpetiar. Balperne lunitagtige, i unore fanten fwarts agtige od farft lubna genom langa, bruna ods fwarta hår. 2nftgtet ods melanfroppen ofwaıl mörfbruna, hårige. 2(6bomen ofwan mörffrun, unbertill gulfrun. Bröjtet likßs6runt, hưrigt.

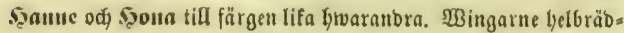
babe, ofwan fwart= eller gråbruna meb eller utan fwarta punf. ter motjwarande unbre fibans oceller. Franfarne lywita. Un= bertill är grunbfärgen lfujare brunagtigts eller gulagtigtcgrå, tillfölie af fort tilltridt lubb. Frantwingarme hafwa mot ut= fanten $1-3$ oceller, bwarbera med lywit pupill ord omgifne of en gul ring. De Gafre hafwa fem bylifa, af bwilfa 2:ne fitta

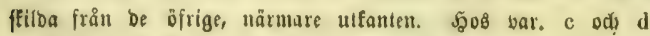
äro ocellerne erfatte genom byita punfter. 
Sarven är finluben, huitgrå méb en brun, fmal linea längs rhggen, fyvilfen på be fräntre frgmenterne àr aftruten od otb̧o. lig. Den lefwer på Poa aunua, Milium effusum m. กิ. anbra gräeflag, od är i rörelife blott om aftonen od natten. Dfiaft öfmernvintrar han od) ionryuppas föllanbe wår. \$upau, jom år fort od, lfusbrun med ntirfare ftref, lar länga winģfibor od fort abbomeñ.

\section{Sligtet: Eipinephele. (Hüв, $H_{-S}$.)}

(llipparchia Farr. Ocus. Salgıus LATr. BolSd. Zett. Erabia Dalm.)

Antennæ longioros, extrorsum hand crarsiores, Clava indistincta.

Palpi longitudinem capitis superantes, subadscendentes, apicibus subnutantibus, compressi, triarliculati. Articulus medius reliquos conjunctos longituslino superans, ad basin basali fero yracilior, in modio dilatatus. exinde subito attenuatus, apice subacuminato; ultimus cylindricus, gracilis, apicn acuminato, basali longitudino regualis. Artirulus medius ut otiam basalis infra pilis longissimis, divaricatis denso instructus; ultimus pilis subdistantibus, in pilositate reliquorum non latens.

\section{Oeull nudi.}

Pedes antici breves, gracillimi, in pilositate pectoris hand occulti, nudi; fomora, libiæe et larsi æq̣ı graciles sunt; tırsi uniarticulati. Fonora reliquorum femine infra nuda; articuli tarsorum ut in genore præcodente, sed articulus quintus quartum longiludino haud superat, Calcaria libiarum validil. Alæ rotundatw, dentatie, prasertim posticx. flargo interior posticaruin ad angulum ani excisus. Colluta discoidalis anticarum subaperta.

Costa ilarum ut in genero precedente, sed costa transversa anticarum inter costam 5 ot 6 exacto arcuata. Interval- 
lum basalo inter costas alarum antirarum 8 of 9 vix duplo majus, quam inter covam 7 ol 8. Ramulus coste prima poslicarum tunidiate rotundata indictus.

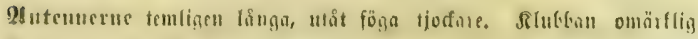

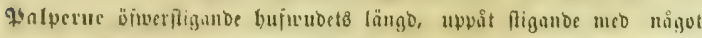
lanonde inetjar, fammantruste, trelebabe: mederfta leden längre

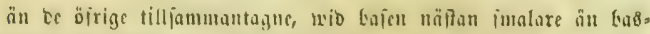
leden, bå mibten bucb, bereiter buftigt affunalnande, ipetfifg; fifta

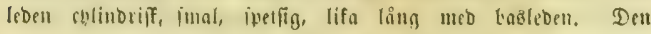

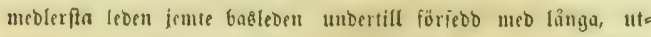
iverribe, täsn thir: ben fifta, med nigot frin burarambra ffilon bair, late bold $i$ be öfrigas birrighet.

Ëponen nafna.

Töterme : de främre forta, ganffa |mula, föga bolda $i$ fröftets bå riglet, nafua; lör, tifier ody tarfer lita fmala; turferne enter

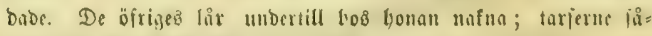
fom fyos föregäende flägle, men femte leden fnappt längle, än Den fierbe. Tibiernes fporrar ftacfa.

23innarur runbabe, tandade, ifunnerbei be fafre. Bafwingarnes inbre fant wio onalbörnet utfaren. Fanumingarmes biffält Galfīppet.

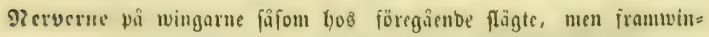

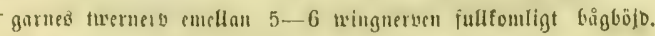
8-9 nerverme pi frambinģarne wio bajen fitda från limaraho Dra genom ots fnappt bubbelt ftörce mellantum än $7-8$ wiแgs nerverne. (S)enen till bafwingarneß förfta nerb anthob genom en rumbab upyimäluning

2fi betta flägte fimnes lifalcbes blott ent ant inom wå fyalfös gränjor:

Shatrergrais-Ryjabilen (Epinophelo janira Lin.)

Alis dentatis, fuscis, anticis teminæe macula flava, in utroque sexu ocyllo; posticis sublus fusco-grisois, postorius pallidioribus, punctis $1-3$ luscis, L. al. exp. $45-52$ millim. 
WBingurne tanbabe, be fränte hos fionan meb en gul fläd, hos Gaba fönen neeb ell ocell; De bafre unbertifl brungra, utat lfujare meb 1-3 bruna punfter. \&. ค. wingip. $1 \frac{18 / 16}{16}-2 \frac{1}{8}$ t.

Syn. Papilio janira Lin. F. Sv. 276. 1053. mas. P. jurlina Liı. 1:. Sv, 276, 10322, fem. P. janira Vabr. E. S. III. 1. 24. 572. P. jurlina Hibu Pap. t. 36. f. 161. 162. Ilipparehia jonira techs. Sichno 1. 1. 218. Erchoia llalm. Pilp. Sv. 83. 13. Satyrius Zett. It:- Lapp. 904. 14. Ciodart of Duponch. Leppid. I. 151. pl. 7. serxi. I. 1. Epinephele II-S.Toxt I. p. 83.

Qtum. Pap. jurtina llïbn. І. с. 1. 36. f. 160. tillbör, iåfou göri. lielf i terten ujwlbier, Pap. eudora Filhr, bivilfen ife förcfommer $i$ Siverige eller Norige.

Bar. b. Bafwingarne ofwan od) unbertill buyitgula, bå febnare făllet med twenne mörfa punfter, mas. ( 22. 个. 104. 105.)

3ar. c. WBingarne bytritgula; be fränte ofwan med en ocell och ber bafom 2-3 röbgula, aplinga flä̊far, unbertill $i$ biften röbguta.

Syn. Papilio brigilla Ijugnh. Act. Holar. 1799. p. 147. t. II. 11. 3. I. 6. 7. (ent. originalsedemular, jom för=

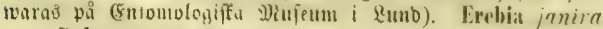
var. Dalim. P. Sv, 83, 13 obs.

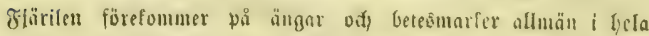

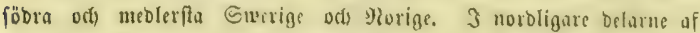
wår balfö blifwer ban fälij̧ntaxe (3etr.). Sguru lögt mot nurden

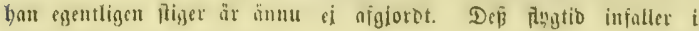
Suli o(t) 2ugufti mainaber.

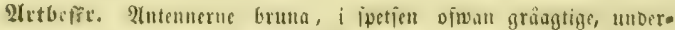
till fëtunse $i$ gult. Walpetne grâbrum, umbertill luona, meto gria od) fivarla lair. 2(nitgtet ody mellanfroppen parft bewurna med mörfbruna fars. 2fooumen ofwan mörfbrun, undertill gulagtig.

5anue : wingarue runbioe, be bafre i ufanten ftarfare tau: Dabe. Eranjarme liufa. 2Ha wingarme ofwalt mörfinum med

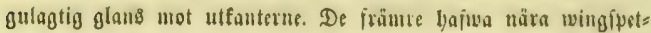


fen en frart, med bivit pupla forfero ocell, gurilfen omgifwes of ell röbgul ring. Itnbertifl ăro be 1 bela biffen odragula, $i$ framfanten od, utfanten brungsigt gri, faunt propobe meb occll, motimaranbe ofre fibans. Baftringarne äro unbertil frin ba. fell till mibten möıft brungrå, berefter ljufare meb $1-3$ mörfruna punfter, gnarbera omgiōne med en gulagtig ring. (P. janira Lin. I. c.)

Syounu lifnar till grunbäargen hannen, men bar i biften på frumisingarno birefiba en ftörre cller minbre rötgul, af grunb färgen belad fäuf, ody fiörre ocell, lywilfen ofra $i$ bufre fanten är åfölio af en minore. Bufuringarne bnfiua unbertill ett liufare banb, Gwilfet uppfommer berigenom aft färgen uämare utfanten är mö:fare, näpan lifa möıf lom wio bafen, 3 detra band finnas mer dler minbre tybliga punfer lifnaube Gannens. $P$. (jurlina lin. I. c.)

Qaryen är fintjårig, grön med hisit floolinea ods lefuer på åıffilliga gräßarter. (Efter alt bufiwa ofwerwintrat blir ban fölfanbe wår, i Mal eller Guni, papn, furiffen är gröngul med jrartbruma firef på twingffidorne od twenne fpetjar i bufwubet.

\section{Slägtet: Santyrus. (Latr.) Boisd. H一S.}

\section{(llipparchia Fabr. Ocns. Erebia Dalss.)}

Antennx longiores, clavate. Clara olongato-ovata, doterminata, compressa, apice rotundata.

Palpi longitudinem capitis non stperantes, compressi, subadscondentes, apicibus nutantihus, triarticulati. Articulus medius lungissimu", longitudine reliquos conjunctos lonģo suporans, subtumidus (ad basin nempe basali latitudine requanc, sed in medio tatior, fero inflatus, ot apicem versus allenuatus); ultimus non apice ipsa medii, sod toieri ejus antico propius insortus, roliquis gracilior, basali nonihil brovior, ox- 
acte oblongo-ovatus. Articulus medius et basalis infra pilis densis instructus; nltimus in pilositate oorum foro absconditus.

\section{Oculi nudi.}

Pedes aulici hreves, gracilos, maris pilosissimi, feminæ pilis parcius ciliatis; tarsi u:iarticulati. Femora roliguorum infra pilosa; tibia tarnique dentibus sat üstinetis muniti; calcaria tibiarum valida; articulus tarsorum primus tribus seguenlihus conjunctis longiludino xquali, secundus tertio, hie tamen quarto, quinto foro wequanti, longior.

Alæ anticx acuminatx, intogra; posticx rotundati, dontatx, margine interiore inlegro, ad anģulum ani rotundato.

Costa: alarum ut in genere precedente, sed costa anlicarum Iransversa intor costam 4 et 5 in medio sxpissime interrupta, tumidiato tantum connoxa. Intervallum basalo inter costas alarum anticarum \& et 9 xque magnum ac inter costam 7 ot 8 . Ramulus costæ posticarum piimæ tumiditate arcuata, acuminata, indictus.

Shteuncrue temligen långa, föriebbe meb flubba. Denna är ap̧ångt: äggformig, befrümb, fammantrhdt, $\{$ fpetien rumbio.

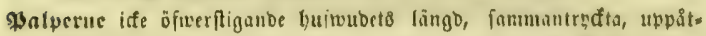
fliganbe meb lutande fpetiar, trelebabe: mebleriza liben ganfa lång, $i$ längb twiba of wergånenbe de öfrige lillfammantagne, nh้s got froullu (wid bafen nemligen lifa brco med basleben, men på mibten brebare, náftan upptlåft, odi) mot fpetien afimals nanbe); ben fifta ife infogab $i$ fieline ipetfen of ben mederfis, utan uämare benncs fränze fant, fmalare ä be ofrige, fortare

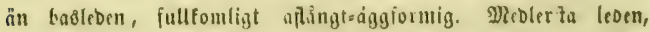
iente basteden, unbertill förjedo med tärthende băr; Den fifta nâftan bold $i$ be öfrigas gårighet.

\section{shouen nafna.}

Fottezue : De främre forta, fmala, hos bannen ganffa bårige, hoo bonan förfebde mid glefare fantbår; tarjerne enlebabe. De ôf rigas lår unbertill lyårige; tibier och tarfer utruftube med gans 
ffa thbliga tänber; tifiernes fporrar flarfa; tarfernes förifta leb

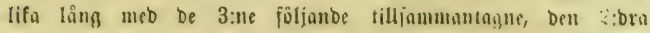
längre än ben 3:ble odh bema längre än ben 4:be, buxilfen ĩ näpan lifa meb ben 5 :te.

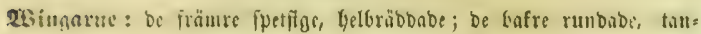
babe, i inbre fantell bela ody wib analtöntet runbabe.

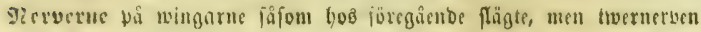

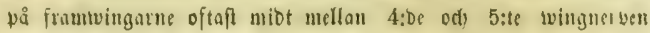
aftruten, blotf faumonfunten genom en upyfwälthing. Framo whingarmes 7,8 od) 9 nerver wid linfen ffilda genou ett lifu flort

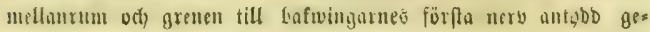
nom en fröjo odi fpetfï uppitwällning.

Drfia betta fägte reprejenterno invu Sfanbinavien af cn enba art:

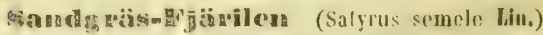

Alis dantatis, luseis fascia indetermenati uaculari fulva; antici utrinçue occellis duobu, posticis supra unico. L. al. exp. $52-55$ millim.

Wingarme tambabe, bruna meb ett af flärfar leftåendi, gult

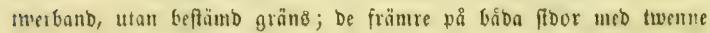
osellex, be bafre ofwin med en. 2. e. wingip. $2 \frac{1}{8}-2 \frac{1}{4}$.

Syn. Papilio semele Lin. F, Sv. 276. 10bi. Nabr. E. S. III. 1. 232. $72 \%$. Iliiln. P'ap. t. 31. 1. 143. 144. Toxi. p. 25. 15. Ilipparehia 0 chs. Schm. I. I. p. 197. IV. 20. 6. Erebia Malm. P'ap. Sv. 79. 4. Satyrus Zett. lus. Lapp. 901. 2. Goul. ot Dup. Lepid. 1. 139. pl, 7. tert. T. 1. II-S. Text 1. p. 76. t. 40. I. 182 var.

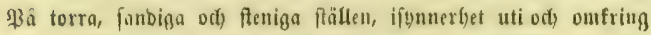
luanfogax, är benna art ganffa allmän i jöbra ody mederfta Swes rige, atminfone änba till Stodbolm. I Siorige förefommer ben

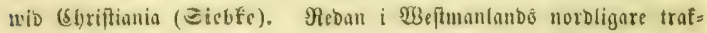
ter Görjar ben blifwa fälligm (Frectid)i.) od̆ aftager fedan $i$ mảngo ia alt ben inom 2eappmarferne är gumfera rar (3ett.). Dép Flugtio

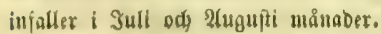


21tbeffr. SIntennerne bruna. \$alperne gråbruma, unbertil

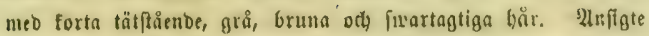
o(h) Glesia brungrå, bårige. Methanfroppen brunluben, med mörf linea längs nibten. 2lbbomen ofran brun, unbertill grå= agtig. Bröftet mórfgrått, ludet. Fötterne bruna, terfnabe meb fwart od) grått.

Sanuc: Bafurimgarne tanbabe $i$ gltre fonten. Framuins garne naggot tillipetfade. Srunbfärgen ofwan fmutfigt fiwarts brun, meb ett ofragult genom be mörfare nerverne i pera fläfar Deladt twerGand nära utfanterne, lywilfet inåt utan beftänd

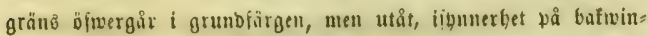
garne, begränfå af en mörfare linea. Stunbom är betta band mera otpbligt ori ofwerbraget med brunt. 3 Gandet baima frommingarne twenne wibt froin fwaranbra filba oceller, of hrvilfa ben främre är föriedo med grwit puvill, od bafırinjarne närmare analböruet en minbre, äjwen med lusit puxill föriebo, yndifen lifwäl flunbou är otholig. Unbertill äro iramsingarme wio bajen röbgula, mot utfanten ofragula, i jramfanten of roingipetjen brungrâ, marmorerabe med fwart, brunt ord buritt, ory $i$ utfanten bruna meb en på fiera fällen aforuten juart linca. Deellerne äro fåfom på öfre fibau, men fumbum fimuro ben bafre blott antyod, eller fafung ben helt od billet. Baf= reingarme äro marmorerabe of fwart, brunt od busitt, bof löper ofwer mibten ett metenbele renare hwitt, wågigt banb. WSin= garneß franfar äro lywita med frattagtiga eller bruma flä̊ar.

Syounn lifnar Gannen till färgen.

\section{Slägtet : Chiomobas. Boss, II-S.}

(Salyrus Lath. Zett. Hipparchia Fabir. Ochs. Erehia Das.,.) Antenne longioro:, extrorsum sensim crasioros, clavam clongatam, gracilem, indeterminatam constituentes.

Palpi longiludinem capitis superantes, subadscendentis, Iriarticulati, Articulus medius longissimus, roliquis conjunclis lon- 
gior, apicem versus sensim attenuatus, pilis brovibus divaricatis infra instruclus; ultimus minutissimus, basali brevior, ovatus, apico rolundalo, ad basin latitudine modio fere xquali:, in pilositate reliquorum vix absconditus.

\section{Oeuli nudi.}

Pedes autici brevos pilis longis divaricatis; fomora nonnihil incrassala, somper libiis tarsisque conjunctis broviora; tarsi tibiis aut longiores aut illis xqualos, uniariculati, acuminali. Fomora reliquorum infra hirla; tibix ut in genere praccdente, ut otiam articuli tarorum; articulus quintus tamen tertio cum quarto longitudino foro æqualis.

Ala integre, posticx vix Jontala. Cellula discoidalis dimidiam parlem alæe somper longo superat; anticarum rlausa.?

Costo alarum ut in genero pracedente, sed costa tantum decima anticarum ad basin inflata. Costa transvorsa anticarum ot inter costam 4 ot 5 et inter 5 et 6 arcuata ut literam S fero mentiet; ad 5 normalis. Ramulus eosto prima posticarum brevis, fere rectus, apice abrupto obtuso.

2lutcuncrue temligen långa, usât fmåningom tjỡare, bilbanbe en lång, funal od) obeftämb flubba.

\$alperne öfwergiende Gufwubeţ längb, uppåtpiganbe, trelebabe: neblerfta teben ganfa lång, längre än be öfrige tiufamman. tagne, mot fpetfen fmoiningont affmulnande, unbertill förjebb meb forta, ulfperrabe bi̊r; ben fifta ganffa liten, fortare än baŝles Den, äggtormig, I fpetfen runbab, wio bajen näftan lifa breb fom bell meblerfa, fuappt dolo $i$ be öfrigas bårigbet.

Sboucn nafno.

Tớterue: be frånre forta, ned lånja utperrabe bår; låren något forfa, altib fortace än tibier odj tarier tiljammantagna; tar. ferne antingen längre äı, efler lifa långa meb tibierne, enlebabe, fpetfiga. De öfrigas lår unbertill lubna; tibierne fajom bos fỏregående flägte, åfwenโå tarfernes leber; ben femte är lifmäl näftan lifalång jom ben 4:be ody 3:ble tiufammantagne. 


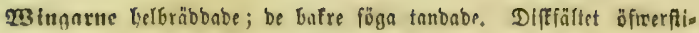
ger alltib rriba wingens balfwa bel; be fränres flutet.

Meryerue tå ningarme fåfoum loo föregâenbe flägte, men blott 10:be nerben på framwingarne wib bafen uppblåft. De fräntres swer: nerb bölo både emellan 4 od 5 od emellan 5 od 6 nerberne, fî att ben lifnar boffafioen $S$; wid 5 :te nerven normal. Sres nen tifl förfta nerven på bafwingarme fort, näftan rät, i fpets fen twert trubbig:

Dib unbantag of en enoa art, fom fimmes på 2riperne $i$ jobbra (Europa, tillfior bettn flägte norra odk) norbligafte belarne af curopa, Ifien od) 2(merifa, ber be leima i fleltrafternę ffogar od̆ färr, od) upveftiga tilloçumed änba tifl inögränfen. Sfanbinabicn räfnar tilf fin Jauna treme arter, af hreilfa ingen förefommer $i$ föbra och meblerfta Siverige ợ fnappt nảgon fliger Getubligt nebon Dowre i Norige.

\section{q. Gulbruna Gräs- Tärilen (Chionobas norna Thlog.)}

Alis subdentatis, fusco-testaceis, fascia dilutiore lata, anticis utrinque plerumguo ocellatis, posticis sxpe ocello unico, infra cinereo luscoque marmoritis, lascia dentata saturatiori. L. al. exp. 45-55 millim.

Wingarne fmastanbate, frungula, meb ett breot linfare banb; be främre på bảba fitoor merendels med oceller; be bafre ofta med en enba, unbertill marmorerabe meb grått od) brunt, famt forrfebde med ett mörfare tanbabt banb. \&. c. mingip. $1 \frac{13 / 10}{2}-2 \frac{1}{4}$ t. Bar. a. $\left\{\begin{array}{l}\text { Alis anticis ulrinque ocellis } 3 \text {, posticis supra } 1-2, \\ \text { infra punclo minuto. } \\ \text { gramwingurne på biba fibor meb } 3 \text { ocellet, de fafre } \\ \text { ofman meb } 1-2, \text { unbertill meb en liten punft. }\end{array}\right.$ Słn. Papilio norna Thbg. Diss. Ins. Sv. 2. p. 36. var. a. lliibu. Pap, t. 152. f, 765. 766. Satyrns Zett. Ins, Lapp. P. 901. 4. Yar. c. Chiomobas Boisd. Icon. pI. 36. r. 4-6. Satyrus Godart ot Dupouch. Lepid. I. 205. pl. 31. f. 4-5, 
Bar. b. Framningarme tå bion fibor med 3 oceller; be bafre utan oceller.

Syn. Satyrus norna Zett, I. c. var. d.

Bar. c. $\begin{gathered}\text { Alis anticis utrinque ocollis 2, posticis supra unico. } \\ \text { Framuingarne pia bibar fiosr med } 2 \text { oceller; bafwine } \\ \text { garne med en. }\end{gathered}$

Syn. Papilio norna llïbn. Pap. t. 152. 1. 763. 764. Ilipparchia Ochs. Schim. I. 1. 201. 17. Erebia Dalm. Pap. Sv. 80, 6. Satyrus Lett. I. c. var. a. Chionobas II-S. p. 69 .

Bar. d. $\begin{aligned} & \text { Alis anticis utrinqun orellis } 2 \text {; posticis inocellatis. } \\ & \text { Eramuringurne pai boiba floor meb } 2 \text { oceller; be Gafre } \\ & \text { utan ocell. }\end{aligned}$

Syn. Papillo norna Thbg. I. c. var. b. Satyrus Zett. I. c. var. b.

Alis anticis utrinquo ocollo unico; posticis ocello

3ar. o. $\{$ aut unico att nullo,

frrammingarne pi̊ båda floor meb en ocell; be bafre antingen neb en eller ingen.

Syn. Papilio hilda Quensel. Act. Holm. 1791. 272. 1. 9. I. 7. ¿. Satyrus norna Zett. 1. c. Var. e. (an Chionobas ocno II-S. T'ext. p. 71. tab. 27. 123. 124?)

Obs. Chinoh. oeno Boist. lcon. pl. 39. f. 4-6 a. Ch. Oenone II-S. I. c. differo videtur. Vide infra syn. ad $\mathrm{Ch}$. bore.

Bar. 1. $\left\{\begin{array}{l}\text { Alis supra inocollatis; auticis intordum infra ocello } \\ \text { unico, absoloto. } \\ \text { WBingarne ofman utan ocefler; be främre unberpounom } \\ \text { unbertill med ell, otbolig ocell. }\end{array}\right.$ Syn. Papilio norna. Thbg. 1. c. var. g. Satyrus Zett. I. r. var. I.

Denna $i$ ampenbe till frorlefen od ocellernes antal mąet was rieranbe fiäril, förefommer $i$ fả̆t od) lägre belägna ăagar uti \&appf fă

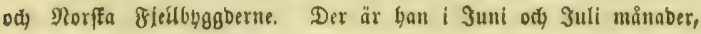




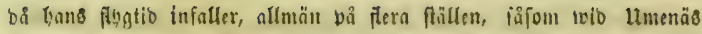

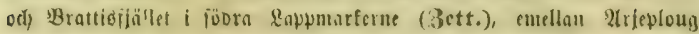

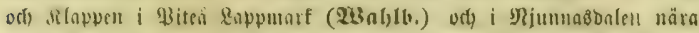
Quiffiof i \&ulci \&appmarf, men ifbunctlet webnom St. Diwe,

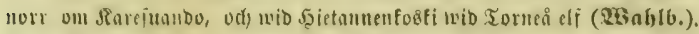

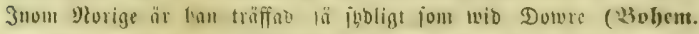

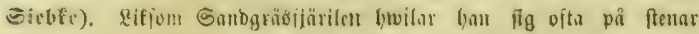
o(i) träbftammar:

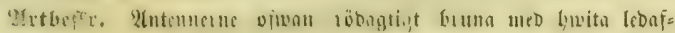

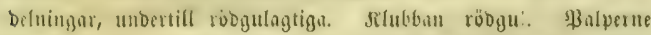

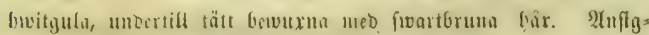
tet ludet of ficurtbunt. Nheflanfroppen fetwuren med längre,

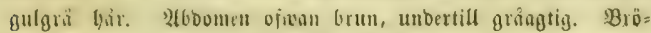
fett mörft Grun=lıbet. Fötterne guloruna.

GBingarne ofwan irin toten utofiwer mibten gulbruna med ett brebt röbgult, mürfare cller blefare banb läng utfanterne, bwilfa

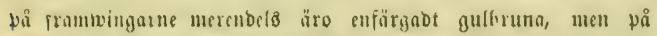
fufivingane vita prnobo mco röbgula därfar. Framuvingarne unbertill bleft röbgula med gulbrun anftrufning i bifent eller bruns

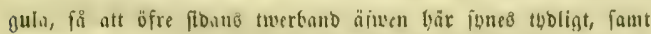

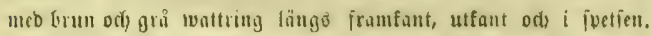
Bifningarne undertill marmorernbe med frunt ods bwitt, meb

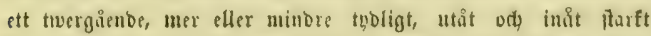
taubnot, mörfare bant. Dả Detta är tyoligt fynę pă hwatbera

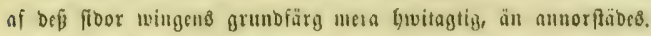

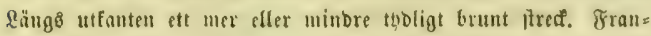
jarme lywitgula med brunagtiga häfar. De mic on bwit punft fôricbo occllerne vaticra till antal, fafon ofunföre blifwit wifaot.

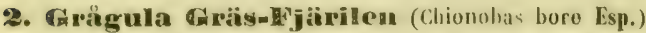

Alis ix subdentatis, griseo-ochracuis, anticis sublus in diseo fusco irroratis, posticis subfus griseo luscoq.eo marmoralis, faseia transwersa saturatioro, plus minus distincta. L. al. oxp. 45-b0 millim. 
Mingarne fnappt fmåtanbabe, gråguln; be frânure unbertill : biffen meb brana atomer: De bafre lutbertill marntorerabe neb grått od brunt, med elt mörfare, ner eller minbre tybligt twerband $\mathfrak{~}$. c. wingfp. $1^{13 / 1 / 6}-21 / 16$ t.

Syn. Papilio borc Esp. Schm. 1. T. 100, cont. 55. f. 1. p. 37. llïbu. Рap, 1, 29, f. 134-136. Texi. 24. 13. Ilipparchia Ochs. Schim. 1. 1. 205. 19. (oxcl, syn. Thiunb.) Erebia llalm. Pap. Sv. 80. 7. (excl. syn. Thunb.) Satyrus Zett. Ins. Lapp. 902. 7 Dup. Lepid. Suppl. I. 209. (oxcl. syn. Thunb.) pl. 32. f. 1-2. Chionobas Boisd. Icon. p. 193. 1. 37. f. 1-3 (excl. sỵn. Thunb.) II-S. Text. i.p. 70. pl. 26. f. 119-122. Papil o norna Quens. Acl. Holm. 1791. 274. t. 10. 1. 12. Pap. formunalus Nabr. E. S. III. 1. 214. 670. Chionsbas Oeno Doistl. Icon. pag. 195. pl. 39. f. 4-6. Satyrus oeno Dup. Lepisl. Suppl. I. 307. pl. 49. 1-3. Chionobas taygete II-S. tab. 24. \&. 112-115.?

obs. Ch. oeno Iboisd, corto est eadem spocies ac Ch. bore Esp., colore obscurioro in pagina inforioro alarum posticarum tantum diversa, quod nullo modo speciem destinctam, sed variclatem tantum designal, ut e speciminis numerosis, eodom tompore of loco collectis of a me collatis, ot ex analogia congenerum salis apparot. Chionobas also Boist. Icon. p. 197. pl. 40. f. 1. 2. nee multum abludero videtur, sed a mo non visum ad spociem eardem eum roforro nolui. Ch. oeno II-S. I. e., ocello in apice alarum ánticarum a specie ejtrdom nominis Boisduvali sino dubio diversus varietati minori $\mathrm{Ch}$. norna ost simillimus, ot ad illam speciem dubius relatus.

Denna art är en af Sfanbinnviens fäajuntafte fiärilar od bits tills endaft funnen på berģsplateaun af St. Dime emeilan 68-69 grabenв volgojjo, i Torneå Eapumarf nära Torneå elf, ber ßrof.

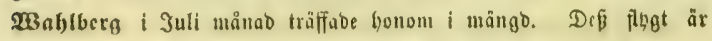
sung od owicy få att man utan fwåriglyet fan bemågtiga fig bowarie exemplar, fom man fair fe.

Irtbefr. 2tntennerne Guitgra med brunagtiga Icbafbelningar. Rlubban röbgul, ofwan brunffuggab, i gtterfta jpetien brunagtig. 
Balperne bivitgrå, unbertia betwuxna meb fwartbruna hår. 21nfigte od fulloror grålubne. MRellanfroppen gulbrun, luben. 2k6. bomen ofwan furart, simerpubrab med gulfrunt od lewuren macb glefa går; undertill left gulbrun. Bröftet fivartbiuns, lu= bet. Fötterne fwitgrå meb bruna atomer. Råren unbertill lubo af täta, fwartoruna biår.

Sqaure: wingarnc ofwan grågula, mer eller minore ftòs tanbe $i$ Grunt. Framivingarme ofwan från roten länģ fram: fanten imalt gråagtige mo bruna afomer, $i$ ivingipetien od ut tanten äfwenlsbes meo bruna atomer; läng8 ben jebuate fynas ftundom äiwen olpbliga rōbgula flăafar. Bafuingarne frăn ba= fen utöfwer mibten monrfare brunagtige, förorfafabt af unbre fibans mörfare färg, fom ber fpnes igenom; längs utfanten ett

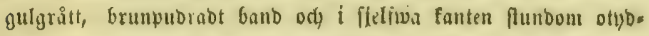
liga, brumagtige, Galfmånformiga fläffar. Framuingarme unbers till gulgrů, längs framfanten od) twert öfwer wingipetīen grås agtiga meb bruna orh jwartagtiga atonter orh junffrect; jäbana finnae äfwen $i$ bif̌̃en; wingbajen branagtig. Bafwingarne limitgrå, öfwer bela gtan murmorerabe med froart odj brunt, . Gwilfet är tâtaft mot bafen; öfwer wingarmes biff går ett af fyopabe jwarta ody bruna atomer bilbabt, ftundom med ofriga tefuingen fammanfiytanbe ody berigentom otobligt, Gand, fom utåt od init mer cller minore begränfas a f fwart. Eranjarne buvits grå neb bruna fläđar twio nerserneb utlop».

Syoun lifnar bannen, men är $i$ alluänbet fótre, ljujaro gufgrå ofman ody Gar mera afrunbabe twingar. Stunbou fine na8 på bafuingarnce öfre fiba längo utfanterne fpar till bnvitgula fläđåar.

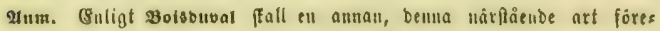
fomuna wib Norb:Bay. Eguru betta uamn af utläiofia forfattare ofta, fffom mau fan fiuna, tages coflectiot för afla norbligafle orter inom (Eus roba, är bet bof en mojlighet, att famma art fiumes i gicrfa fimmarfen, Daglact ben hittills unbgitt be refante fwenfe Raturforffores Mbumitfam Get, fum befoft bedfa trafter. Då ben faleber isfe äun fan med fáferget 


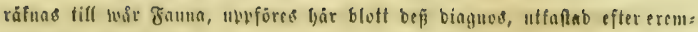
plar fraํ (d)ỏnland:

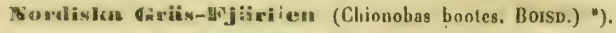

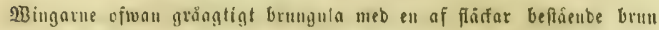

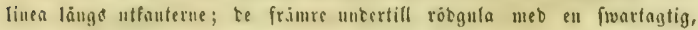
winflab limea twert ofwer tiffen; be lnafre unbertill marmorerabe meb grifte cd brunt, med ett morfare fmalt twerbanb ofwer biffel, wdi en mer eller minbre tyolih, af fläfar befående, brunngtig linea länģ utfanten.

Syn, Cmoxomas booles. BoIsd, Icon. p. 191. pl. 37. f. 4. b. 6. Hippargiia Theitsch, X, 1. 32.

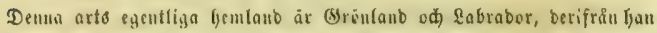
eges $i$ Efantinavifa fanlingar. Enligh soisbuval fall ben äjuen föres

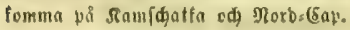

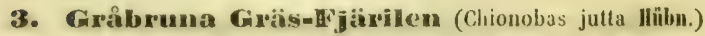

Alis subdentatis griseo-luscis serio macularum fulvarum, antici: utrinque ocellis 1-4, posticis 1-2; his infra cinerascentibus fusco itroratis, fascia obscuriore transvorsa, plus minus distincta. L. al. exp' $50-58$ millim.

Wingarne juåtandabe gråbruma, meb elt rab röbgula Thăfar; be främre ขå båba fiour med $1-4$, de bafre med $1-2$ oceller; be febnare unbertill affegria, brmmamorerabe meb ett mörfate, mer eller minbre thbligt twertunt. R. ๔. wingit. $2 \frac{1}{16}-3 \frac{3}{8}$ t.

Syn. Papilie julla llübn. Pap. t. 120, f. 614.615. Ilipparchia norna iar. Mchs. Schum. I. 1. 202. Satyrus julla Iett. Ins. Japp. 902. ";. Godart el Duponeh. Lepid. Suppl. I. 250. p1. 40. I. 3-5. Chionobas Hoisd. Icon. 187 1. 38. I. 1-4. II-S. Text I 68. pl. 25. I. 116-118. (Chionobas balder IBoist. Icon. 189, 1. 39, \. 1-3. II-S. 68. 1. 79. 1. 384-386. Satyrus Zett. 1. c. 902. 6. Godart et Dupouch. I.ep. Suppl. I. 310, pl. 49, Г. 4. 5. varielas hathd dotincta, secundum oxomplaria nummorosa, a me collata).

-) Spreies lantum ut incola troenlandico nobis eognita; nee in Lapponia, nee in linmatkia (ar an Cap hord) adhue reperta, quantum mili inotuit. 


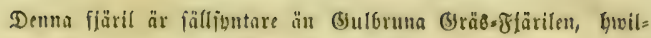
fen ben närmą? lifnat ods funars barietet ben of älore författate

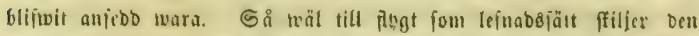

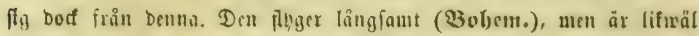

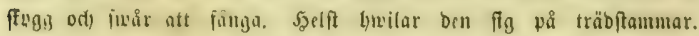
Den f:Dligafie orten inom Smerige, bet ben blimit fumnen är Dre

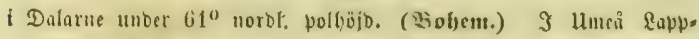
marf funts den mera talıift närn encfele, uti ett färr emellan all= mänแa wägen odh Sgelleforß (Bitt. Dablb. T3ablb.). I Norige är ben funnen fmoliguft wid Golöer (Sicbét). Deß̉ flugtio injaller i Suni od) Suli månaber.

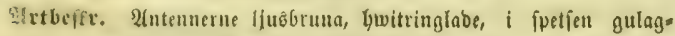
tiga. \$alperne mörforma, unbertill fwarthårige. 2Anftgle ody 1489 1.̈ld fwartbruna, lubna. 2t6oonen oiman mörforun, un=

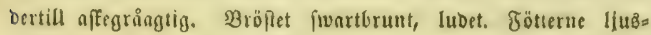
Gruna.

Soatuc: Wingarne ofwan gråbruna; be främre nảgot tillfpetiade $i$ främre ljörnet, ofwan meb $1-4$ runbabe, fiwarta flärfar $i$ en rab anbra röbgula; ben mederfta futnoom liten, flunbou vffia alla vţ̧bliga. Bafwingarne ofwan meb en rab pilformiga eller ovala, fundom otbbliga röbgula flüđar längs utfanten, af Grvilfa 1-2 nära analförnet hajwa brwarbera on

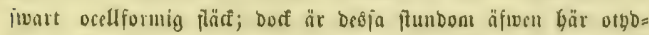
liga. Frammingarme แnoertia läng framfnnten od) unert ö fwer wingiperjen affegrá med imartagsiga eller bruna ftreff od ntomer; i biffen bleft fruma mid fwarta, af gult omgifma, ocell. formiga fläfar, belägı närumare uffanten od) motjwarande öfre ftoans, bof flunbom $i$ antul minbre ăn på bemua. Bafningatne affegrå marmorerade med fina bruma pller fwartagliga ferect; öfwer biffen ett mörfare talloabt Ganb, flunbom (nät grunbfärs gen är mörfare ån slię) omärfligt; mot utfanten 1 -.-2 fivarta, runba ocellifa fläfar, motjwaranbe jfre fiban8, bivardera otyb= ligt omgijne af gult. Ofta är äfwent bär antalet ell minbre ân på ôfre fiban. 
Syonan ftillicr fị ţ till färgen frân bannen blutt beri, att öfre fỉdan är något lífuare brungrå, odh bafwingarnç unore fiba plunbom mörfare, få att twerbandet blir orbabligt.

21 uu. Den fafou färffitb art anfebra varieten: Chionobas balder, fliję fig frâu beu af Boidbuval antagua normaln formen fơr Chionobas julla blott genom olifa antal af ocellerie. Deu förn far fri framuins garne 3 oceller sfman od 2 unbertill, famt pả biba fitone af baftuingurne blett en ocefl. Den fulle od variera mera tifl antalet af frammingarues oceller, men alltio pi bafivingarues jire fion hafwa tweune, men på unbre

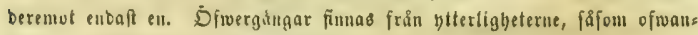
före $i$ artbeferifningeu blifwit twifabt.

\section{Slägtet: Euebra. (DALM.) BoISD, II-S.}

(Satyrus LATR. ZETT. Hipparchia FABR. OCuS.)

Autemne Jongiores, exlrorsum crassio es claram crassam, oblongam, sat determinatam constituentes.

Palpi longitudinem capitis non suporantes, subadscondontes apicibus nutantibus, pilosi, tiarticulati, quoad forma articulorum pro specie bariantes (articulus medius ant conformis, aut in medio latior; ultimus brevis I. Iongior, aut ovalus, aut conicus, aut cylindricus); ultimus tamen somper villosus a reliquis vix distinguondus.

Oculi nudi.

Pelles atutici brevissimi, in pilosilato poctoris plano occulti, tantum foro uno latero pilis longissimis, divaricatis, in maro densius quan in fomina, instrucli, nonihil incrassati; fomora maris aque longa ae tarsi cum libiis; tarsi brevissimi, conici I. ovati; fomora tamen fominx tarsis cum tibiis longiora; tarsi longiorus, gracilos. Tarsi in ntroque soxu uniarticulati. Podos roliqui omnino ut in gonoro procodonto. 
Ala rotundate, jutogra, posticx rarius subdentafe. Cellula discoidalis dimidiam parten alse vix superat.

Costa alarum ut in genere precodonte, sed rosta anticarum transersa inter costam 4 et 5 recia, ad hanc plorumque ovanoseons, of inter 5 ot 6 iterum recla l. parum arcuala; costa ipsa ad costam ala 5 semper geniculata. Ramulus coste primx poslicarum, bresis, curvalus, apice plerumque acuminato.

Uuteuncruc temligen långa, utảt tjocfure, bilbanbe en tlof, af= lång, temligen beftämb llubba.

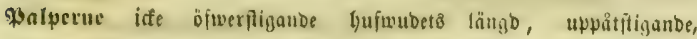
urcb lutande fpetiar, bảrige, treledabe, i anjeende till leoernes form f,os ipecies variernnce (meblerfta leben antingen lifiormig sller pai mibten brebure; ben fifta fort cller längre, antingen

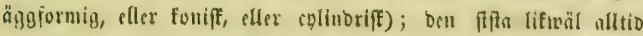
bårig, Enappt fitilibar från be dofrige.

Şoucu natna.

Füterue: De främre ganféa forti, allbeles boloa $i$ bröftets barige bet, näftan Glott wå ena floan fürf́cobe ned lânga, utiperrade Ljår, bos bannen tätnxe än bos bonan, migot tilltiofnabe; yan= nenछ lår lita långa med taries ocb tibier; tarierne gunfa forta, foniffa cllex ăggformiga; Gonan lax beremor längre ân tarịer odi) tibier, tarferme längre od imala. Tarjerne enledabe. De oีfrige fötterne fâjom tyos föregående flägte.

x३ingarac runbabc, bela, be tafre jällan funtnubabe. Difffältet ofwerftiger fnapt wingens Galiwa längb.

शerverue på wingarne fåjom yos föregåenbe flägte, men be främs reßs twernerv pmellan 4 od) 5 wingnerwen räl, wid benn mer: enbelह förfwimnande, od) cmellan 5 od) 6 återigen rit eller fögn böld; bela twermerven wio 5 umingusven alltio winflab. (S)renen till förfta uerven på bafmingarne fort, fiöf, mercubelo tillipetfab.

De till Desta flägte hürande arfer, fürefomma meb unbantag af en enba, enoaft i nown trafterne of Giserige od Norige. En del 
af bem figa boc äiuen ned $i$ be meblerfh belarne af lanbet. Dele ffogar, bele tînt aro beras wiftelieurt od de fürefomma blott $i$ en generation om året.

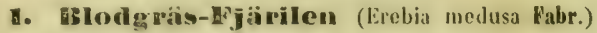

Alis integri hrunneo-fuscis concoloribus, ocollis subquatuor in fascia maculari rufa. L. al. exp. 40-44 millim.

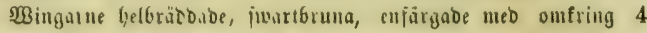

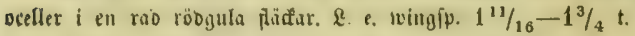

Syn. Papillo medusa Vahr. E. S. III. 1. 235.734. Hühı. Pap. 1. 43. f. 103. 104. Tost. 34. 47. Hipparchia Ochs. Schum. 1. I. 273. IV. 22. Satyrus Zett. Ins. Lapp. 903. 9. Gollart et Bupouch. Lepid. II. 110. pl. 15. fo 5. 6. *). Erebia Dalm. P’up. Sv. 81. 9. II-S. Toxi. I. 66.

פov. $\left\{\begin{array}{c}\text { Alarum fascia maculari fulva nulla, ocollis } \\ \text { præsertim in alis posticis chsolelis. }\end{array}\right.$ WBingarne utan röogula flücarar, ocellerne ifiln= nerbet på Gafuingarne ouärfliga.

Satyrus medusa Zeit. I. c. var. b.

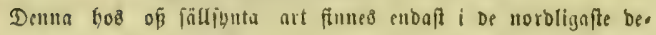
larne af Eappmarferna od äjwen ber temligen fällynt. WBio Rares juando i Tornsi \&appmarf är ben funnen af \$rof. Setterfitcot,

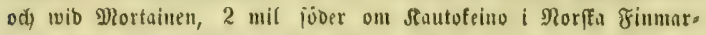
fen unber $69^{\circ}$ volfüjb, fauns ben på en äng temligen talrift of

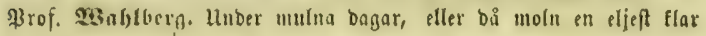
bag bortifummer folen, dölier ben fig $i$ gräfot. Figgtiden intrâffar i Juni månab.

Artbefs:. 2(ntennesme opiwan fwartbruna, unbertill huvitagtigs. selubtun ofwan fmurlorun, i fpetjen röbgul, unbertill gnvitagtig.

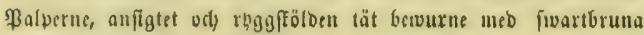

-) Marula rufa alarum sulicarum sunt in speciniubus, a mo collatis, exacte rotundala, wec orato-nblonge ut in figuris Godarti el Duponcheli, preterea sunt etian ocelli duo in apice anticarum reliquis majores, nec is æquales ut in fipuris memoratis. 
bår. 26bomen ofiwan fwart meb fwartbruna glefa bån, unbers till ljụare mörfbrun. BBoöftet fwartbruแt, luot. Fötterne gmitguln.

Şanแe: WBingarne ofwan enfärgabt fwartbrunn; be früntre meb 2-4 (bögft fällu 5) fwarta ocellex, gyrarbera belägne i

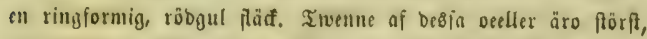
lywaranbra närftảenbe o(l) belägna wio wingiperfen, fant flunbon bwarbera förjedo nted buit pupill. Bafuingarme bafwa $1-4$ blinba oceller, lifa frambingarnes. Unbertill äro alla mingarme lifalebes fwartbruna; De främre äro lifură $i$ biffelt röbagtigt anlupna, i twingiperfen meb twenne fiörse, alltib liwitpupillerabe oceller, motfurande ofre floans ods bafom beşa anbra minore, till antulet warieranbe, men alltio belägne $i$ röbgula fläctar, få fom på öfre fỉan. Bufwingarne haj̄wa läng̊ utfanten $1-4$ fmörre fwarta oceller, bwarbera omgifne af en röbgul, fmal ring, od) någre förfebde med guvit pupill. Eranfarme oftwall o(h) un= bertill funartboma.

Seonan filiper fig till färgen frin lyumen beti, att be robo= gula ringarne omfring ocellerne äı på uringarnes bion fioor blefare, famt bafwingame unbertill, oftare än bos bannen, ftrar framom ocellerme ody $i$ utfanten minore mürffruma, än wio kas fen, Gwarigestom ett liujare bano bilbar, intagande bufre treb. febelen of wingen, beri odja vcellerme äro belügne.

- Earven, fom lefwer pa Panicum sanguinale*) Geffrifuco

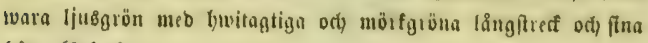
ฤดำ. (0chs.)

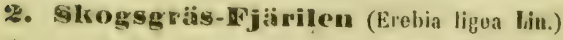

Alis subdontalis, nigro-brunnei-, fascia rufo-ferruginea; anticis utrinquo ocellis 3 -4; posticis ocollis 3 ot inlra plerumqquo maculis albis. L. al. oxp. 43-47 millim.

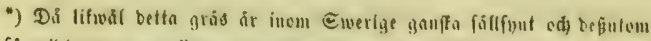

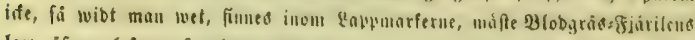
larb áfuen lefiea of anbra gràzacter. 
Wingarne fmitantode, funartbruna med elt roftrobt band; be

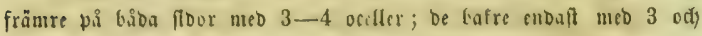

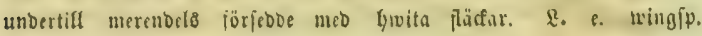
$13 / 4-115 / 16$ t.

Syn. Papilio liqen Lin. li. S. 275. 10:50. Palur. E. S. III. 1. 234 . 732. Mübn. Pap. 1. 49. 1. 225. 226. 227. llipparehia Odis. Schom. I. I. 283. IV. 23. Truitseh. X. 1. 52. Satyrus Zett. Ins. Lapp. 903. 10. Ciodart et Diponch. I.epist. II. 96. p). 13. 1. 1. 2. Erebia Dalm. Pap. Sv. 81. 10. H-S. Text. I. 63.

Bat. a. $\left\{\begin{array}{l}\text { Alis posticis infra obscuro-hrunneis unicoloribus, ocel- } \\ \text { lis } 3-4 \text { et striga alla brevi. } \\ \text { Bufuringarme unbertill mösferuma, enfärgabe, meb } 3-4 \\ \text { oceller ods ett fort, buritt fred. }\end{array}\right.$

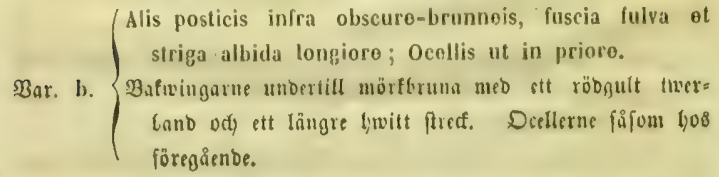
War. c. Alis posticis infra obscure-brunneis, ad basin pallidiWar. c. BBafwingarne unbertill mörfbruna, mib bajen blefare, ted? nabe med ett hywitt twerband ; ocellerne omårlliga.

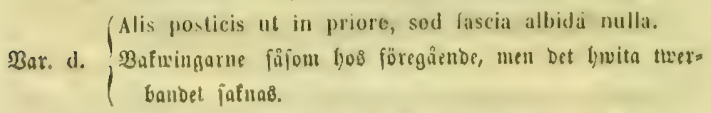

Fुärilen förefoumer $i$ รuli vdy 2lugufti mannaber uti ffogarne

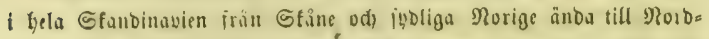
(5av. Doof är lyan fillan funt $i$ Gfaine, ment beremot $i$ mederfia Smerige ganfa allmän, fajom $i$ D̈itergö̀bland tvio (Suјum

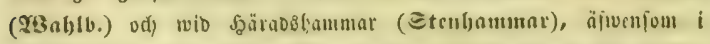

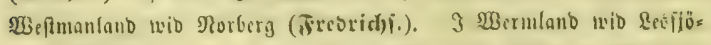

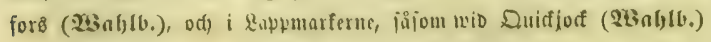
od) omfring Tomeå (3ett.) är lyan ganffa talrif. I Norige fins

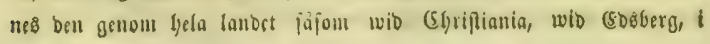




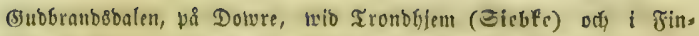

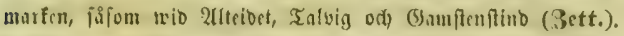

Artbefe. 2(ntemnerme ofwaแ lịtêbruna, unbertill gulagtigt grå. Balperne gulagtigt grå, undertill fmartfiarige. Sroppen fwart. Nogofölden fwartorunt luben. Fötterne mörfbruna, på främre fiban gulagtigt grå.

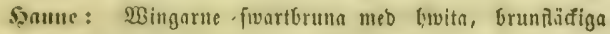
franfar. De främre prebón ofwan, nüra uffanten, meb ett Grebt, af nerberne telabt, roftröbt Gand, lywart $3-4$ oceller med cller utan gyrita fupiller funns. De emenne of besfa oceller äro buraranbra närftionbe, belägna $i$ wingipetien od) oítaft nära bet roftröbis banbets inbre faut; be twenne öfrige, af fusilfa

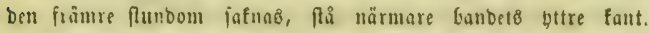

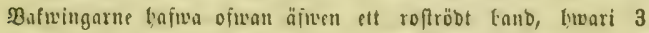
oceller meb tyrita pupiller finnas; bof äro flumbom tmenne af be\&fa otşbliga. Frammingarne äro undettill liujare juartbruna,

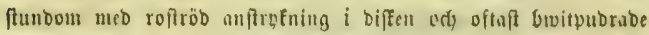
i främre fanten, midt för bet roftröba tmerbanbet. Detta bar occllerne faijom the offe piban. Baftringarme variera fäjom ofwanfôre Glıf̧it nänubt.

Soun lifnar lannen, ment ầ liufare till färgen, ody mera yarieranbe till bafwingarnes unbre fiba.

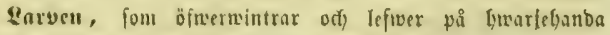

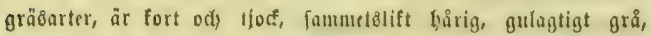

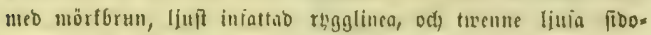
linier, famt furarta luftbil. Fömanolingen fer i Dal.

\$uppan, fom ligger fri pai forben eller bland gräerötter, fyar furartbruna tedningar od farf punftcring.

\section{Gulringlade Gris-Ejärilen (Ertbia embla Thbg.)}

Alis subdentatis, brunne-luscis, anticis supra ocellis 3 -4 nigris, rulo circumcinctis, inlra 2-4; posticis supra ocollis 3- 4, ruto-circumeinclis, infra maculis duobus albis, punctisque 2-3 nigris, obsoletis, ad marginom cineroo-adspersum. L. al. exp. 50-55 millim. 
গुSingarue fmåtanbabe, mörffruna, be främre ofiman mę $3-4$ fwartn oceller, oumgine af töbs, unbertill 2-4; be bafre ofran meb 3-4 oceller, ongifue af robt, unbertill meb 2 brita fäcfar, or wib bell meb affegrnitt vubrabe utfanten $2-3$ fwarta, othbliga punf: ter. \&. f. mingip. $21 / 16-21 / 4$ t.

Syn. Papilio embla Thumb. Diss. Iıs. Sv. 2. 38. 1. 8. 8. (fig. incorr.) Quens. Act. Holm. 1791. 1. I. 9. ศ. 1. 2. (ligg. incorr.) Ilipparchia Ochs. Schm. I. I. 287. IV. 23. Treitsch. X, 1. 54. (oxcl. syn. Auclor, Pap. grielix ot disa). Eruhia Ialm. Pap. Sv. 82. 12, II-S Toxt I. 61. Satyrus Zett. ItIs. Lapp. 904. 12. Papilio ethus Ralir, E. S. III. 1. 217. 680. Pap. dioxippe Ilübn. Pap. 1. 105. 1. 538. 539. Satyrus Godart ot Duponch. Suppl. 1. p. 230. pl. 36. f. 1. 2.

Denna, få mibt man bittills fänner, för Swerige egenbonlliga art båller fig på lägrc belägna trafter $i$ be norbliga lanbffapernes nebre frogeregion, men iffe uti egentliga färs. Flghtiben infoller i కuni od) Jutli månater. Den fodligafte provins, ghwareft arten föres fonumer, lär wara Dalarne, ber bell of Sucufel blef funnen wib Gerna ody Elfibalen. $\Im$ Eappmarferne àr ben träffab på fôljande

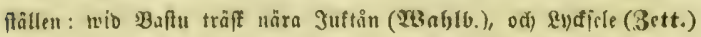

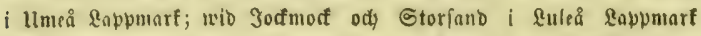

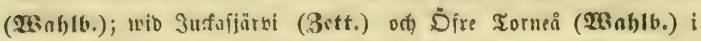

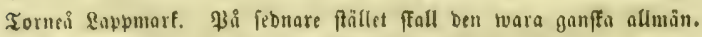
Den fall ärnen una junnen twid Sarefuando of Grape. Dita Gyilar bell flg på tröbplanmarne.

Ifterffe. *) 2(ntennerne ofman bruna med hasita ringar, unber: till frwitagtiga meb fruna ringar. Rlubban röbgul meb brun=

-) Descr. Antente supra dilute brunnex annulis alhis, infra alhidas annulis hrunneis. Clara fulra apice brunaeo. Palpi, fascies et thorax dense brumeo-hirsuti. Ahdomen supra obsrurius, infra dilutius fuscum pilis brevibus obsitum. Pectus fusco-hirsulum, Pedes flavidi.

Mas. Alxe supra brunuen-fusex, omnes prope maryinem exteriorem circulis 3-4, invicem plus minus separatis, rufo-fulvis, in quibus ocelli niggri, alarum anticarum maximi, stepissime albo-pupiliati, arsunt; ocellus 


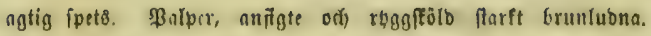
2160omen ofman môrfare, unbertilf Ifufare brum, forthairig. Bgöftet mơlftrunt fubet, Eötterne röbgulagtige.

sonute: WBingarme of wan mörfbruna, hw. rbera meb 3-4, nära utfanten belägne, röbßgula ringar, Gmari finmab fmarta, funbom p\& frammingarne furitpupillerabe oceller, of fwilfa be

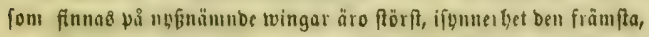
fom har bubbel fupill. Framuringarne äro unbertill lifalebeø mörforuna meo 2-4 oceller, af fammin form ody färg, fom öfre fibans; wingipetjarme pubrabe meb affegraitt. Bgafwingarne un= bertill mörfare bruns än jranuxingarne, i utfanten gri̊pubrabe oda mé 2-3 otnbliga firnita punfter, motimaranbe lifa många af oire fibans oceller; offwer biffen går clt otgoligt ođ matt mågigt, brunt frred, fafom bnvilfet en fãd funnes $i$ wingens främre fant od) ent annan i biffen. Franfarne äro brunagtigt grน̊ med bruna päđơr.

Sounat ftilfer fig från banmen beri, att grunbfärgen àr lifuare brun ach Eaftwingarne pil unbre floan länģ utfonten mera gråpubrabe. Stundom, cl)uru fälan, bafưa äiwen fram. uringarne vå unbre fiban on femte, liten ody otģblig osell nåra infonten od) lafuingarne på famua fiba en topblig röbgul ring omfrimg en eller amuan of be fwarta punfterne.

primus anticarum reliquis major, interdum pupillis duobus allis præditus. Alæ antica infra brunnen-fusce ocellis $2-4, n t$ in pagina superioro coloratis formatisque, et apicibus cinereo-adspersis. Alw posticw infra obscure brunneo-fusec margine exteriore cinereo-adsperso, punclisque 2-3 nigris, ocellis pagina superioris respondentihus; in disen striga obsoleta, undulata brunnea el pone illam macula alba ad marginem anticum et alia in medio. Fimbria brunneo-prisescens maculis fuscis.

Femina cotore dilutioro et margine exteriore alarum postiearum infro densius alque latius cinereo-adsperso a mare differl. Ala antica in pagina inferiore ocellum quintum minulum el ahsoletum prope marpinem interiorem, el postico in pagina eadem circulum rufo-fulrum obsoletum circum punctun unum l, altrum nigrum interdum, quamsis rarissime, gerunt. 
4. Svartstreckarle Gris-Ejirilen (Erchia disa Thbg)

Alis subdentatis brunneo-fuscis, antici: ulringue ocellis $\mathbf{b}-\mathbf{b}$, rufo circumeinctis; posticis supra unicoloribus, infra griseocinernis faseia media lata, donfata strigaque arcuatil fuscis. $L$. al. exp. 50-53 millim.

Wingurne jmåtumbabe, mörf6runa; be frümre Đå Gába flobor med 4 oceller, omgifme af röbt; be bufre ofwan enfärgabe, unbertill mörft affegrå med eft brebt, tumbabt frumt bano odt elt böfot firce of jamma färg. \&. e. mingโp. $2 \frac{3}{16}-2 \frac{3}{16}$ t.

Syn. Papilio disa Thunb. Diss, Ins. Sı, 2. 37. Satyrus Zatt. Ins, Lapp. 904. 13, Erebia H-S. Text. 1. 6 1. Pap. griela Valor. E. S. III. 1. 236. 23\%. Ilinbu. Pap. t. 49. f. 228. 229. Pap. steno llïbu. 1. 109. f. :561. 562. Pap. gefion quensel. Act. Ilolm. 1791. 270. 1. 9. f. 5. 6. (fiz. incorr.) Erebia embla var. b. Dalm. Pap. Sr. 82, 12. Erebia embla Boisd. Icon. 162. p. 8. t. 33. 1. 1-3. Satyrus fiodart et Buponch. L.epid. Suppl. I. 232. pl. 36, r. 3. 4.

Denna art tilltion be ftora färren ody mprome uti Streriges

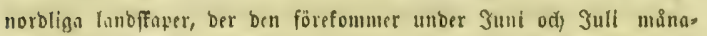
ber. 3 Umeâ (anpmarf är ben funnen alumin emellan Sorfele odi Mroffenoine (Zis nblb.) ody wid Wojmfiön (Brtt.), famt i Torneå

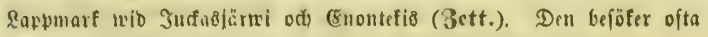
blonmorne af Rubus chamamorus.

Artucitr. *). 2Intennerne ofwan bruna meb hywita ringar, un= bertil gulagtiga, âfwenfom bela Elutban. Balper, anfigte ods

-) Descr. Antenne supra dilute brunnex onnulis athis, infra flavidx. Clasa tota flarn. Palpi, fascies el thorax dense brunneo-lirsuti. Abdomen supra obseurius, intra dilutius fuscum. Peetus fusco-hirsutum. Pedes flavo-albidi.

Mas. Ala supra brunneo-fusen, subnitentes: posticx unicolores, sed antica in margine anteriore et in apice cinereo adspersa et prope marginem exteriorem maculis $4-b$ nipris, rolundatis, unaquaque circulo lato, rufu, cum proximo suo conjuncto, circumcincta, Harum macularum antice sunt approximatx; omnes tamen pupillis carent. Ala antice ut in 


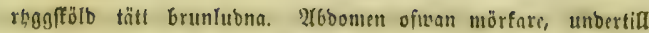

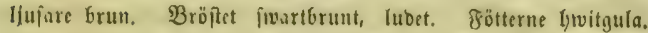

Saanue: $\mathfrak{B}$ Bingarne ofwan mürf6runa, naggot glänfande; be Lafre enfïrgabe, ment be fräure länge iramfanten odi) $i$ ipetfen Hubrabe mid gaåt, famt nära utfanten prubbe meb 4-5 jwarta fläfar, Gywarbera omgliwen of en röbgul, breb ring, fom fam.

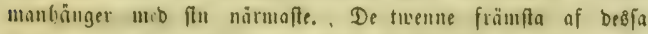
flisfor äro fumar:ubra närftâenbe, men afla utan pupiller. Uns bertill lyafma frammingarne fammta grunbfärg ods tefning fom pio offe fiban, men äro fnebt birer wingipetfen od) längs ut= fanten gripuorabe jant probobe med minore oceller. Bafwin= garne äro på fommua floa mörft affegrå, elfer på brum botten tätt grioubribe, o(t) bafiwa ofiver biffen ett brunt utåt od) inåt mörft begränjabt, tanbabt tand, faut mibt emcllan betta od IIffanten ett wågigt trmergående freef of famma färg. Eranfarne äro ฟå bâbn fibor fmutfigt turita med bruna fläctar.

Sounan frilier fịg från hannen genom liufare srun fârg ofwan od mobun, famt lfuвిgufare ringar omfring ocellerne på framtwingarne.

\section{Fjellgrais-Fjärilen (Erobia manto Pabr.)}

Alis intogris nigro-fuscis, anticis supra fascia maculari ferruginon plus miuus distincta, utrinque punctis 2-4 nizris; poslicis suprat aut unicoloribus, aut punctis $1-3$ nigris, infra cinerascentibus fascia transversa, dentata saturatiori in disco. L. al. oxp. $43-49$ millim.

pagina superiore, ita in inferiore coloralx, in apice tamen et in margine exferiore cineren adspersa, it ocellos minores gerunt. Ala posticw in pagina inferiore sunt frisco-rinerex 1 . in colore biunnen-fuseo dense rinereo-adsperse, ef in disco fasciam latam, extra et ilatra obscure maryinatam et dentatam, fuscam ot in medio inter haue el marginem exteriorem strigan, Iranswersam arcuatam undulalauque ejustem coloris gerunt. Fimbria utrinque sordide alba, fusco-maculata,

Femina a mare colore utrinque dilutinre ef ocellis alarum anticarum circulis flavidioribus circumcinctis tantum differt. 
WBingarne byelteräbbabe, jwartbruna, be främre ofwan meb ett af flärtor beitåenbe, mer eller minbre tmoligt, roffärgabt banb od) på Gåba foor 2-4 inarta punfter; be frafre ofwan antingen enjürgabe, eller meb $1-3$ jiwarta punfter; unbertill gragatige meb ett mörfate, tanbabt twertanb ofwer biffen. \&. e. wingip. $1 \frac{3}{10}-2$ t.

Syn. Papillo manto Kabr. E. S. III. 1. 231. 722. llibu. Рap. ใ. 45. f. 207. 208. t. 101. 1. 512. 513. 514. Ilipparchia Oehs. Schm. 1. 1. 296. IV. 23. Erebia Dalm, Pap. Sv. 82. 11. H-S. Text. 1. 59. Satyrus Zett. Ins. Lapp. 903. 11. God, et Dup. Lepid. II. 12\%. pl. 17. f. 7. 8. Pap. la ppona Thumb. Diss. Ins. Sv. 2. 37. f. 66. Pap. erina Nabr. E. S. 1II. 237. 739.

Demns art, fom albrig träffas i jubalpinffa trafter, år allnän i beta Norige od inom Suverige från ody meb norra Dalarme änba

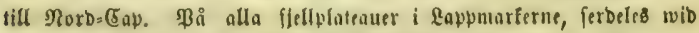

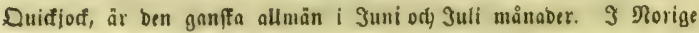
går ben änba néb till (s) riftiania.

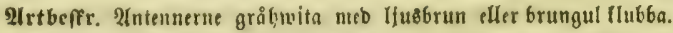
Balperne lifu\&bruna, lubna. Bröft od mellanrsigg framtill Ifu\&s brunt, men för öfrigt mörfbruut lubne. 216oonen ofman mörfs brun, unbertill gulagtig, eller hrwitgrå, fortbårig. Fötterne mer eller minbre gråagtige.

Syaume: Wingarne Gelbräbbabe, of wan fwartbruna, glän= fanbe, fiunbom, i ipnnerbet hos fåbana exemplar, fom flugit en lăngre tio, ljufare bruma. De främre hafwa mot litfanten ett rofifärgabt, mer eller minbre tholigt, brcot banb, beri wanligen 4, mera fällan blott 2, fwarta meb gulagtiga ringar omgifne, pupiller, fafnanbe ocellifa punfter, finnas, broaraf be twenne

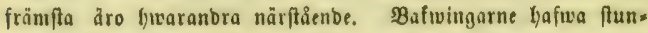
Dom alot utfanten 2-3 fwarta puntter, brvilfa bwarbera bỏgft fällan är ongifne of en rofffärgab rilig. Unbertill äro frams roingarne $i$ Gela biffen roffärgade, i främre od zttre fanten blångtigt grå, längs inove fanten brunagtiga, od bajwa fmarta punfter motfivaranbe öre floans. WBingurnes franjar äro buvits agtiga. Bafningurne äro unbertill wid bajen flartt grå 
Iubna, men till färgtedึingen för ôfrigt på fôljanbe fätt buries ranbe:

1. Bufruingarne blångtigtraftegrå meb ett af tweenne taggiga, mer eller minbre tybliga, bruna linier inneflutet mörfare ballo öfwer biften, ody för öfrigt öpwerftroboa meb brunage tiga atomer.

2. §if föregåenbe, men har trib bttre fanten $1-3$ imarta punfter.

3. Bafrwingarne enfärgabt grâlruma med ets of tmemne tagsiga, bruna linier inneflutet mörfure taerbenb öfwer bifen, od wio roten hrwitagtiga.

4. Sif "uärmaft föregående, men ueb blott on tuggig, fuubon omärflig brun linen öjuer biffen ods $1-3$ frarta punfter nära utfanten.

Sconan lifnar till färgen bannen, utom beri, aft bafwin. garnes unbre fton är mera gulgrå. Dod är bon till besia mingarb tedning unberfafad faminia sariationer fom bannen.

\section{Trib. Nymphalides. BoIsD.}

Pedes tantum qualuor postici gressorii, antici 2 spurii, broves, dobilos, sxpius pilosi.

Cellula discoidalis alarum posticarum plane aperla I. costa transversa subspuria, tenui, ant abrupta aut continua clausa. Cellula anticarum aut costa abrupta somiclausa, aut costa tonuissima, spuria, plane clausa.

Margo anticus alarum anticarum costis 2 ot ramulis duobus torliæ, I. costis 3 el ramulo uno quartie, nompe ramulis duobus I. tantum uno coslæ alarum septime of costis 8-9, 1. 8-10, exdentitur.

Costa alarum posticarum semper $9 ; 1$ o Irunco communi cum 2-4 ogredions, ramulm breviorom I. longiorem, reclum 1. arcuatum in lobum basalem emiltens; 8 -9 o basi egre- 
dienirs; anticarum aut 9 aut 10 (7 tri- 1. quadri-ramosa) ad basin semper invicem requales; 1 o basi egrediens.

Ale postice pro receptiono abdominis canaliculatæ.

Larra cylindrica, ant ramoso-spinosa, aut tuberculis carnosis, spinas montientibus, obsita.

Pupa turberculala, apico anali susponsa.

Fotterne: blott be fscra fafre åro gångfötter; främfia traret forta, rubimentära, oftaft hårige.

Diffältet på bafningarne alloeles ïpwet eller of tillfutet af en falf, fin, antingen afbruten, aller continuerling tmernerb. De främres biffält antingen baliffuct genom ell afbrulen twemerb, eller of fullfomígt flutct misbelfs ent fin, falft nerb.

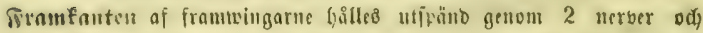
2 gressar af ent treble sller genom tre nerber ods en gren af ell fierbe, nentigen genom en eller turi grenar af 7:be wings uerven od genom 8: be od) 9:be eller 8:be-10:de nerverme.

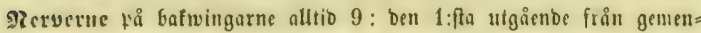
fam flam mid bell 2:bra-4:be od) utịnubunde en längre eller fortare, rät eller fröft gren ât ixingen rotlob; 8-9 ut: gi̊enbe från bajen. De främres antingen 9 eller 10 (7:be tres eller furgreniß), wib bafen finsemellan Jifiormige; ben 1 ; fta från bafen af wingen.

33afivingarue för abbomenв แpptaganbe Gilbanbe en ränna mebelift inbre fanteno nebbölning.

Earven chlinbrift, antingen med areniga taggar, eller förfebo meb föttiga utwerser, fom lifna taggar.

\$upau fantig, fafträngande meb analtaggen.

Störita belen af hitforanoe arter fyafiwa en flubbranbe och tems ligen fnabb flugt, fant lefwa fajom larber pii lägre Dicotblebo= ner. Şögft få angripa träbens lọ̈. Färgerne yos fiärilarme äro Gufwubjafligen fwart od röbgult, fälian fuwitt på twingarneß Dire flob. 
Ormpens till múr Fruma föranbe genera funna på iöljanbe fătt frilthô fråı lowaranora:

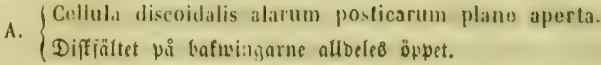

1. $\left\{\begin{array}{l}\text { Costx alarum anticarum } 10 \\ \text { Nerverne på framuingarme } 10\end{array}\right\}$ Limenitis.

2. $\left\{\begin{array}{l}\text { Costx alarum anticarum 9. } \\ \text { Netuerne på frammingarme 9. }\end{array}\right\}$ Nelitaa.

13. Collula discoidalis alarum posticarum costa spuria clausa.

Diffiätet ya bofuringasne flutet genom en falft twernero.

1. $\left\{\begin{array}{c}\text { Costæ alarum anticarum } 9-10 ; \text { costa } \\ \text { transversa sat distincta, subcrassa. } \\ \text { Nerverne på framiningarne } 9-10 ; \text { tuer }= \\ \text { nerven ganfia tublig, temiligen farf. }\end{array}\right\}$

Argynnis.

2. Costæ alarum anticarum 10 ; costa trans-

Perverne ఎå frambingarne 10 ; twerner. Vanessa. ven mer cller minde iörfivimande, fin.

Slägtet : Iimenitis. Fabr. Ochs. H-S.

Antenna longiores, rigidie, oxtrorsum crassiores. Clava gracilis, oblonga, cylindrica, indeterminata, a scapo non distinguenda.

Palpi broves, longiludinom capitis vix superantes, supra ot infra brovius pilosi, ad lalora squamali, apicibus divergentibus, triarticulati. Arliculus modius longizsimus, compressus, lam tus articulus; ultimus haud anguslior, brovi-simus (prino brevior), conoidous apico obtuso.

\section{0euli nudi.}

Peles anlici gracilos, femina longioros nud, maris pilis brovibus cilitiati, tar:ibus inartienatis. Fomora coleromm, presortim posticorum, infra prope basin brovo hirsula; libja 
dentibus parvis of ad apicom spinis duabus brovibus instructe; articulus tarsorum primus tortio of quarto conjunetis longitudise æqualis.

Ala postica dentalx. Collula discoidalis alarum posticarum plane aporla, anticarum costa spuria clausa.

Costæ alarum posticarum 9: 1- \& o trunco communi suporiori (prima ramulum longiorem, valde rofloxum*) in lobum basalem omittens), 5-7 e trunco communi inferiori, 8-9 - basi ogredientos. Collulæ discoidalis oxilus intor costam 4 et 5 , vostigium costa transversæ non ostendons. Costæ alarum anticarum to ut in genero Vanessa, costa tamen octava cum septima, triramosa, ox oodom loco anguli cellula egreditur. Collula ipsa intor costam 4 of 5 costa spuria tenuissima clausa.

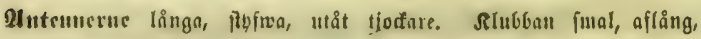
çlinoriff, obeftämb, från ffaftet iffe urftilibar.

\$alperne forta, funppt öfwergående bufwubets längo, ofwatt odg uns bertill forthårige, på fiborne fiällige, meb bivergerande juetfar, trelebabe: meblerfa leden länglt, fammantrustit, breb; fifta leden föga imalare, ganffa fort (fortnre än ben förffa) fonift meb trubbig fpetis.

Souneu nafna.

Fötterue : be främre fmala, yos honan längre od unfua, yos byats nen cilierabe uned forta bair, tarferme olebabe. De ofriges lår, ifthnerbet be bafres, unbertill nära Gafen fortbårige; tibierne föriebde mid forta tänber od, therule forta fporrar i fpetfen; tar: fernes förfia led lifa lång med ben 3:oje odj 4:be tilliammantagna.

23ingarue: be Gatre tanbabe. Difftältst på batwingarne allbeles oppet, på framuvingarme flutet mebelft on falf twernerb.

Rerveruc på bafwingarne $9: 1-4$ utgående jrån öfre nervftain: men (ben förîta ufändande åt rotloben en mydet bafiatböio, längre gren), 5-7 från nebre netuftammen, $8-9$ från เพing:

-) Arcualus, apice marpinem exteriorem spertans. 


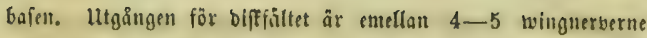
od) wifar of fpâr till twernerb. Framuningarnes nerber 10 fåfom huos yenus Vanessa, men ben 8:be utgår från famma ftâlle i yỏrnet af bifffältet fom ben 7:be, bwifen är tregrenig. Stelfwa bifffältet är flutet af en ganffa fin, falfe, twernerb.

å

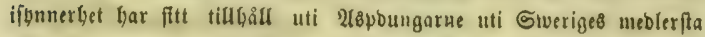
od föbra provinfer:

\section{Asp-Tärilem (Limenitis populi Liu.)}

Alis dentatis, supra nigro-luscis, anticis alto maculatis, posticis albo fascialis; infra luteis alho maculatis fasciatinque, marginihus coerulescontibus. L. al. exp. $75-85$ millim.

Wingarne fmůtandabe, ofwan fwartbruna, be främre bueitîäctige, be bafre med ett bisitt twerbano; unortill röbgula meb busita fäafar och banb, äfivenfom blåagtiga utfanter. \&. e. wingip. $3 \frac{1}{16}-3 \frac{4}{8}$ t.

Syn. Pap. Populi lin. F. Sv. 27\%. 1055. Vabr, E. S. III. 1. 111. 343. Ilübur. Pap. t. 23. f. 108,-110. Limenitis Ochs. Schm. I. 1. 145. IV. 18. Dalm. Pip. Sv. 64. 1. Nymphalis God. pt Dup. Lepid. I. 112. pl. 6. sucund. f. 1. 2. Limenitis II-S. I. p. 43.

Denna wadira art, ell af be förfta till wair Fauna böranbe

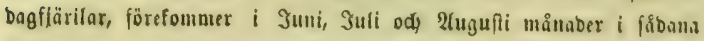

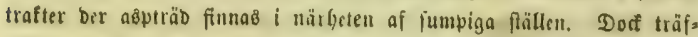

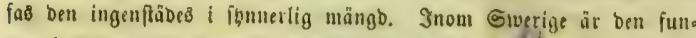

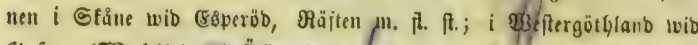
(B)

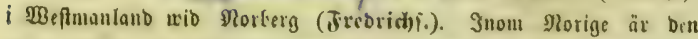
blott träffab i be pobligafte orterme t. ex. wio (5Griftimia, Tönsberg,

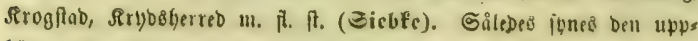
börn outer, 60 eller 61 grab. norbl. polgölo.

21rtbeffr. 2(ntemuerne juratbruma i fpetien röbgulgtiga. ßal. perne ljwitgrå, längg öfre ợ unbre fiban mörforum, D̈gos

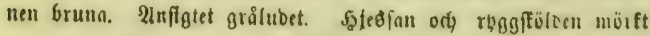
Grunlubne; äjwenfå abbomen ofivan, men unoertif är ben grå. 
agtig. Broffet graitt. Lâren huwitagtige. Tibierne od tarferne Yjusbruna.

Syaute: SBingarne ofwan fwarttruna meb obetublig glans.

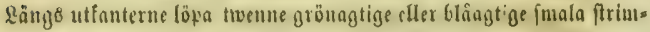

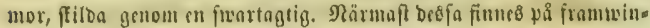
garte en rad otgbliga, men på bafwingarne farfare marferabe od) ftörre ftwarta flisfar, i builfa på be febunare ftr̊ röbgula halfmånar, jom wänba fperfane mot utfanten. Disbligt röb.

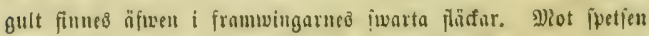

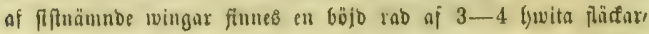
od) berpå fölier twerb öfwer twingen ell flingrande rab af 6 anbra förre od) minbre flaffar, builfa antingen äro rent hywita, eller of mex eller minbre brumpubrabe. $\Im$ biffältet foues en

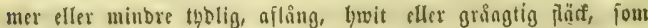
lifuäl frunbom beit od yallet jafnas. D̈ fiwet Gaftwingarne går ett twertano of 7 buita pärfar, ffilba fran lywarando genom be

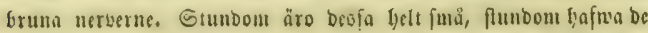
anfonlig frorlef. Undertill äro nlln teingarme söbguln; be fränure lifwäl $i$ inore bäliten (närmaft infanten) fwatagtige. Hufauterne äro jurarta mé twerume Glăagtigt gröna bnub. De fivita päcs farne byafua pai framtwingarne famma läge fom bå öfre fiban, men äro ftorre odl) funbom ftuggabe med blå fürg. WBingroten

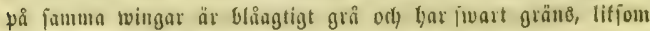
ben lywita Diffläcell. Baftwingarnes rot vech inbre fant är brebl blågrå och en fläđ of jumma färs finnes $i$ diffiältet och) nära

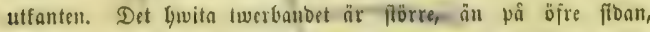

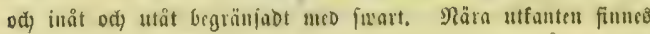
en rab pwarta fläđar.

Souna lifuar bannen, men bar oftaft bet lyvita tuerbanbet på bafwingarnes of re fiba brebare.

Earven, fon lejwer af blaben pâ P'opulus tremula, har bârba

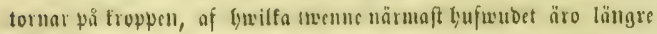

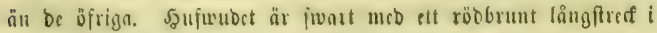
mibten. 4, 6, 8 pdi) 9 froptosingarne äı röbbrula, be ojriga 
gröngula meb brun od frart fuggning. 5 odg 6 fropposins

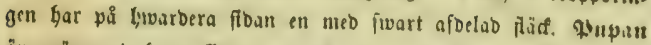
âr grön meb frun faggning od) fivarta punfter. Baton bröft= regionen finues en bilformig, brungul utwert.

\section{Slägtet: Nielitaea. $F_{A B n}$. et Aucr.}

(Argyunis LATR.)

Antenux long:oros, graciles. Clava subturbinata l. pyriformis apice rotundate, in speciminibus exsiccatis excavata.

Palpl capite plerumque longiores, adscendentes apicibus divergentibus, triarticulati. Articulus medius compressus, maximus, hirsulissimus; articulus ullimus basali longiludino æqualis, gracilis, brevius ac adpresse hirsutus.

\section{Oculi nudi.}

Peles autici tenues, ferninæ longiores nudiusculi, maris pilosi, farsibus biarticulatis; femora colerorum infra parco et brove pilosa; tibix dentilus rigidis instructx of ad apicom spinis duabus longis, subvalidis armatx; tarsi כ̋-articulati : arliculus primus longissimus, socundo ot tortio, oliam sepe quarto conjunctis longiludino æquans; secundus et terlius invicem requales, quartus brevissimus omnium, quintus tortio quartoque conjunctis requalis unguibus 4 minoribus armatus.

Alæ subdenticulatx. Collula posticarum plano aperta, anticarum plerumquo semiclausa.

Costæ alarum poslicarum 9: 1-4 o trunco communi superioro (costa prima ramulum aut rectum aut refloxum in lobum basalem emiltons) 5-7 o trunco inferiori, 8-9 o basi egredientos. Collulæ oxitus intor costam 4 et 5 . Costa alarum antiearum 3: 1:ma o basi, 9-4 o trunco communi in- 
teriore, 5 e costa Iransversa, 6-8 e trunco suporiori (6 ef 7 e amgulo collule, bee quadriramosis) of 9 o basi egredientes. Costa transersa, collulam discoidalom claudons, inter coslam 4 et 3 pleramguo alraptia, rarius conlinua tenuissima, vario tamon modo formala*).

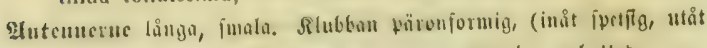
Gred) med afrumbad fuete, bos torfabe excmplar urliolfab.

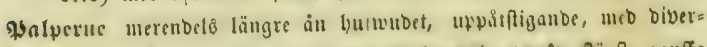

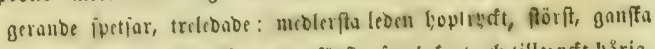

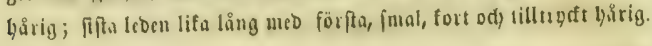
હ̈вouen nafua.

Fotferuc : be frömue fmala, bos fyonan lângre o(c) näftan nafna,

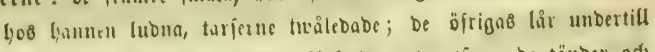
iparjamt forbariza; tibienc föricobe med utjperrabe tänber od i ipetjen temäpnabe med 2:ne linga, temligen fartis fforrar; tarjerne femlebabe: 1:fla leben linglt, lifa lång meb 2:bra ora 3:Dje, ofta äjwen nub 4:De tillinmmantagne; 2:Dra ods 3:ole fingemeflan lifi, 4:De fortaft, $5:$ te lif.a lång med 3:ble od, 4:De silliammantagne ods benäpnad med 4:ra minore flor.

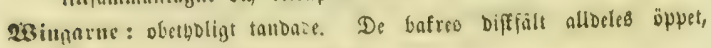
be fränires metendolo balfflutet.

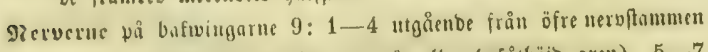
(1:ja fändonbe åt rotloben en rât ellex batâtböjo gren), $5-7$

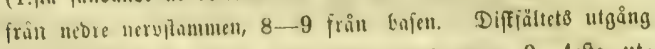
iir encllan $4-5$ nerben. Frantuingarmes nerver $9: 1:$ f̂a, ute gaimbe från bajen, 2-4 frîn nebre netufanmen, 5 te frôn tuernctben, 6--8 frän öfre mervfhumuen (6 od) 7 från böruet ai Diffiältet, Den jebnare furgtenig), 9:be frå bnfen. Tiverner. vill, fou tillfuer biffiältet, är murenbels aforuten emellan 4:De od, 5:te nerven, fällan contimuerlig od ganffa fin, men få olifa jütt formà *).

-) Tabula formain cosle irausreves alarum anticarum exhibens:

A. Cellula discoidalis olarum auticarum clausa; costa transversa inter costam 4 ot 5 continua elsi tcuuissima . . . . . M. maturna. 
De fefta tiff betta flägte ljöranbe arter förcbraga öpwna ängar, ifunnerbet jåbane, fom äro af mera frolänbt beffaffenter. Răgra fả träfo fas på torrare lofaler och endapî en I ffogarne. De förefonuma blotk i ell generation om året od bafwa temligen juabb aligt. Surben âr befläbo mè töttiga, fornlifa utwexter ở) Jeftwer på lägre werter, făllan på träb; alltio $i$ fôrre eller minbre fäแffup.

\section{Boke-Nätfärilen (Holitæa maturna Lin.)}

Alis supra fuscis maculis flavis of fulis, propro marginem exteriorem fasciam formantibus; anticis infra fulvis maculis albis evanescentibus; posticis infra fasciis tribus flavido-allhis, modia linea nigra longitudinali divisa. L. al. exp. 45-50 millim.

WBingarne ofiwall möstoruma meb liueggula od röbgula fätar, Gwilfa nära utfanten filda ett twertand; De främre unbertill röb. gula med lywita föriminnande fiäfar; De bafre undertill med trenne Guvitgula twerband, bet medcrfta belabt mebelfî en lang? gående fwart linea. \&. e. svingiv. $1^{13 / 16}-2 \frac{1}{16}$ t.

Syn. Papilio maturna Lin. F. Sv. 280, 1062. Ochs. Schm. 1. 1. 18. IV. 13. Melitiea ma/urna Ualm. Pap. Sv. 74. 1. Boisd, lcon. p. 110. 1). 23. I. 3. 4. Zett. Ins. Lapp. s09. 1. Godlart of Buponeh. Lopid. Suppl. I. 135. pl. 22. 1. 1-3. II-S. ToxI. 1. 20. 1. 29. f. 132. Pap. cynthia llübn. Pap. t. 1. f. 1.2. ot mysia Tuxt. p. 5. n. 1 .

Obs. M. maturna i Suecia inferiore (Scania et Btelingia) non in Lapponia occurrit, c,ua de calla Gizura llulsneri $598-601$ ad eam relerri non possunt,
ut voluerunt Auctores.

B. Celluls discoidalis alarum anticarum semiclausa: costa transersa (plerumque inter costam \& et 5) abrupla.

1. Costa transversa inter costam 5 el 6 fortiter arcuata M. iduna.

2. Costa transversa inter costam 5 el 6 acute angulata M. artemis.

3. Costa transversa inter costam b et 6 recta 1. M. delia $_{\text {M. alhalia }}$ modice arcuata ......... $\left\{\begin{array}{l}\text { M. alhalia } \\ \text { M. diclynna }\end{array}\right.$ 
Denna ftäril är en of twåra fälligntare o(t) tillyör få wibt bito tills meb füfertyet blifwit utrönt endaft Sweriges fobligafte provinjer,

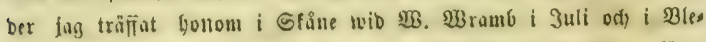

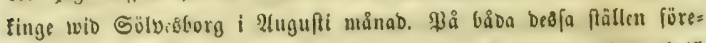
fommer lyan $i$ Gofeftog. Dita builar Gan fị bå botarues blab, belft be bufturtabes. WBio \&und ä lyan af \$rof. Betteriftcot fummen $i$

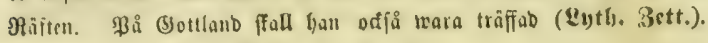
$\Im$ Norige finneß ban iffe, få wibt tag wet.

Srtbefrs. 2ntennerne fruma, unberill röbgulagtiga, ofwan fint busitringlabe. Stubban med gul fpetô. Balperne ofwan fwart= Gruna, unbertill quitgult lubue. NRงgefföldon, äfuenjom aboo.

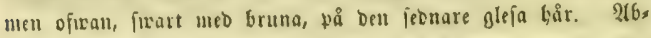

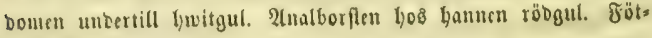
serne töbgulagtige.

Sลanue: WBingarne oftwan mörft 5runa, näf.an fwarta; De främre med ett bredt tiserfuno af $3:$ ne raber olifitora, lius: gula od röbgula fläđar nära utfanten od) anbra täcfar af famma fäıв fpribda $i$ diffen. De flärfar fom bilba medlerfta raden $i$

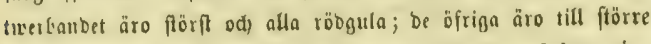
eller minore antal lifusgula. Gạjom fortfăttning af framwin= garnes twerbano gair ett bylift öfixer baftringarne ede beflir af

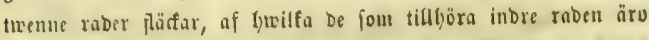

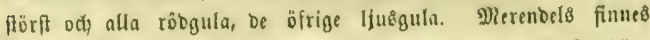
immantiôr betta twerband antyoning till en trebje rad. I bifen

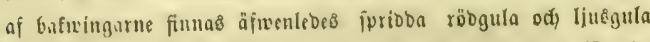

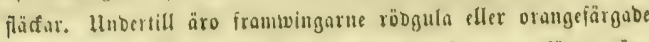
med fwart terfning $i$ biffen od) twerftrect of famma färg; nära

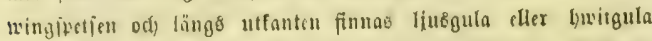
Näcfur, af lywilfa te feonare inat begränjä of fwart. Bafivins

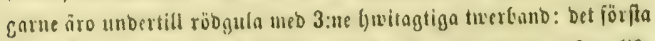
närmaft roten aforutet od Geftående af $3-5$ med fwart omgifne olifformige flücfar; bet 2:bra ofiver mibten, inåt of utåt fwart beo gränfubt ở ti̊ lângoen belabt genom en aftruten fwart linea; bet 3:Dle nära utfanten, befrienbe af Galfmånjormiga, froart bee 


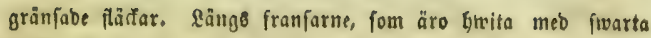
flūđar, löper på ningarnes unbre fiba en fmart linea.

Soman äx pörre än bannen ody har manligen twertanoet på wingarmes öfre fiba brebare än benne.

Earveu, fon har pî froppen föttiga utterter, lifuande tornar, är frrart mé en Dubbel rab gula punfter längo raggen od) en annan enfel lïngs bwarbera fiban. 5ूan lefwer fö̀cträ.

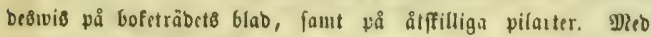

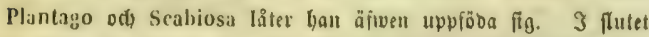
af Dhaj eller börfan af Juni blir ban pupa, feban lyan öfwers nintrat. Denna år grönagtigt husit med fwarta punfter od fareff famt bar på nbominalregionen 7 raber gula, fnappfors miga taggar:

\section{Eappska Näfjäirien (Molitæa iduna Dalm.)}

Alis supra nigro fuscis, fasciis maculariis albis ot obscuro fulvis; anticls infra ut in parte superiore coloratis, posticis fasciis tribus albis maculariis nigro-marginatis, omnibus impunctatis, L. al. exp. 35-40 millim.

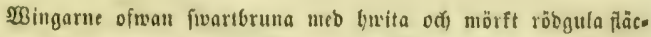
far, fom bilda twerband; be främre unbertill lifa öfre fidan, be bafre med 3:ne twerbano of buvita, fwartfegrämfude fläđar, och fafna fwarta punfter. \&. e. wingiv. $1 \frac{7}{16}-1 \frac{6}{8}$ t.

Syn. Melitzea iduna Dalm. Pap. Sv. 75. 2. Mett. Ins. Lapp. 900. 2. Godart el Mrponch. Lepicl. Suppl. I. 300. pl. 48. F. 3. 4. II-S. Text. I. p. 21. Pap. malurna Hï̈sแ. t. 117, f. 598, 599, 600, 601. Text, p. 5.

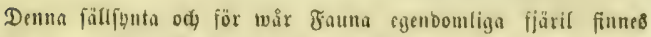
enbaft i norbligare belux af Errenffo sappmarforme, ber ben $i$ när.

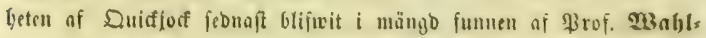

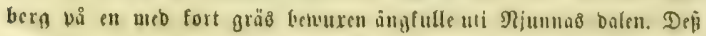
rimgtio infoller $i$ medio (onfring ben 18:De efler 20:be) af Suli แล̊กเดb, 
Qrtbeffr. *). 2(ntennerne ofwan inarta meb Greita lebafbelnin. gar; unberfill röbgulagtige. Slubban fwart mot fpetfen rōbgul. Balverne röbgula, unbertill meb fwarta od̆ röbgula Går. Srops

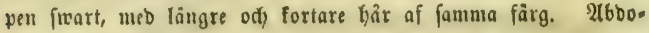
men unbertill wib anus röbgul. Fỏtterme röbgula.

Syaute: Wingarme ofwan froarta, meb fhra af lärfar bes ftående tmerbanb, af Gwilfa hwarannat ät röbgult od hwaran= nat lysist, bod fi att ett röbgult àr uármnft uffanten. (5neflan bet inbre bwita twerfanbet od biffältet pi frambingarne fins nes en buwit, flor fläf, fom belas genom nerverna; mellan bifts fältet od) inbre fonten finnes̊ cn annan af famma färg; i fieliwa

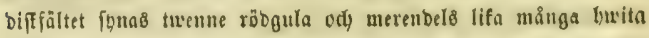

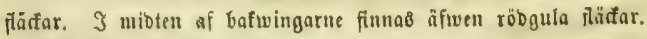
Framivingarne, fom wid bafen äro fwarta, bafwa unbertiál

-) Descr. Antennm supra nigrm arliculis albis; infra fulvida, Clava nigra, versus apicem fulva. Palpi fulvi, infra nigro fulvoque barbati. Corpus nigrum, pilis longhiorihus el brevioribus ejusdem coloris obsitum. Abdomen infra versus anum fulsum. Pedes fulvi.

Has. Alæ supra nigræ I. nigro-fascæ; fasciæ quatuor maculares, alternation fulvo et alba; fascia fulva tamen anargini proxima. Inter fasciam inlernam alarum anticarum albam et cellulam discoidalen est macula magna albs, costis transeuntibus nigris divisa; inter cellulan ef marginem interiorem macula altera ejusdem coloris; in cellula ipsa maculo dur fulsw et plerumque tolidem albo obserrantur. In medio alarum posticarum maculw fulvæ etiam adsunt. Alæ anticœ, que ad basin sunt nigræ, fasciam latam alham per medium et fascias dus fulvas tenuiores, nigro-marginatas, atera alba interjecto, infra rersus marginem gerunt; cellula ipsa maculas ut in parte superiore habet. Also postice ount fulso maculis albis 3-4 irregularibus, nigro marginalis, ad basin, fuscia alba maculari ejusmodi nigro-marginata (rarius linea longiludinali subtilissima, subfusea divisa) in medio et altera, lunata ejusdem coloris ad marginem. Costo alarum omnium suni in parto inferiore nigro. Fimbrio fusco.

Femina, qum plerumque est major, a mare maculis albis is parte superiore alarum minoribus distinguitur.

Hab. rarius in Lapponia; ad Quickjock mense Julio, 
öfrer mibten ett brobt, fywitt twerbanb od, närmare ulfanten turenne anbra fmalare, röbgula ode finartfuntabe, mellant huiffa

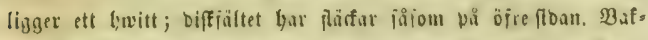

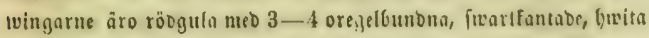

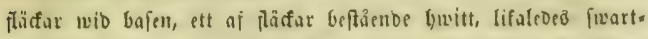
fantadt Gand (fällan längs mibten belind medelft en fin bruns nôtig (inen) öjuer mioten of et ett amuat af famma färg trio uts

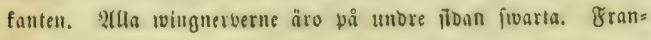
farne bruna.

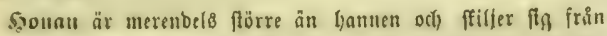
yonom beri, att ofre fibans lavits flärfar äro minbre.

\section{Arenpris-Natfiarilen (Nelitia artomis $\mathbf{W}$. V.)}

Alis supra fuscis maculis flavis fulvisquo; posticis infra fasciis Iribus maculariis albido-favis, utrinque in fascia fulva serie punctorum nigrorum, subtus futoo-marginatorum. L. al. oxp. $31-45$ willim.

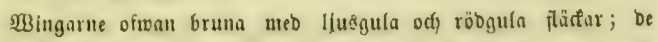
bafre unbertill med 3:ne af Riffar beftiende linitgula tmerband, od på bỉba fibor med en rab jirarta, undertill gulfantube punfter uti ett röbgult tmerbano, \&. e. wingip. $1 \frac{1}{4}-1^{19 / 16}$ t.

Syn. Pap. arlemis W. V. 322 . Vabr. E. S. III. 1. 255. 790. llübn. Pap. t. 1. \&. 4-6. t. 129. f. 653. Text. p. 6. 4. militea 0chs. Schm. I. 1. 24. IV. 13. Godart et Duponeh. I.epid. I. 71. pl. 4. seserml. f. 3. pl. 4. tert. f. 3. Dalm. Pap. Sv, 75. 3. Lett. Ias, Lapp. 900. 3. II-S. Tuxt. 1. 22. Pap. malutina Thumb. Diss. Acad. Vol. III. 45. Pap. maturna Esp. Schiı. I. t. 16. r. 2. p. 209.

2Trten förefonmer fporabift inom Emerige änba upp i Dalarne,

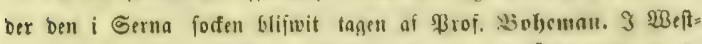
mantund är ben ef fälfibnt omfring Norberg; i D̈pergüllyano är

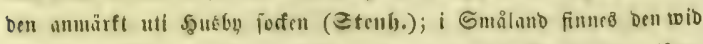

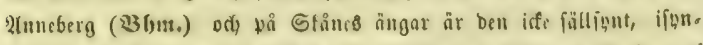

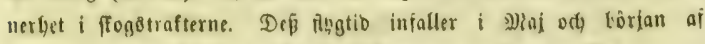

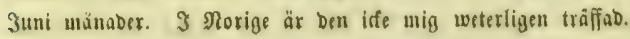


Srtbeffr. 2(ntenuerme fruma meb Emita ringar. Rlubban uns berilll röbgul; fuctẹn ofran af famma färg. Balperne rổ= gult båriga. Nugsfölben of abbumen ofwan med röbgulagtiga

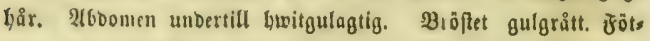
terne rŏbguingtiga.

Scaume odh houn: WBingarne ofwan röbs ellex fiwats bruna med mingga röbgula ợ ljuaggula, tärftåenbe, olifformiga flädor; be bufre meb trenne af pänfar bilbabe tmerbanb nảra utfanten, af luwilfa bet inbre och butte hos bannen, men blott bet feonare hos honan är liwitgult; bet febuare berjente alltio bilbabt af trefantiga, inåt naigot afrumbabe fläfar; bet meblerita od) brebafte är beremot hos båda fönen röbgult ody utgöres af fläcfar, fom ftillas från bmaranbra blott mebelf be mörfa ner, berne ody lafwa bwarbera $i$ mibten en fmart punft. Bå unore floan äro wingarne röbgula; be fränure med talrifa Ifuళ̧gula, ferbeles mot wingfperfen tätpåenoe flĩfar; be bufre hafura trenne liusgula trerband, af lwwilfa bet förfta (nib roten) uts görcs af 3-5 olifftora, irart onglfna flüfin; bet anbra, fom går öfmer biffen, Delas genom mingnerverne $i$ olifformiga fiącs

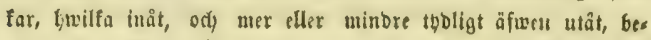
gränfas af fwart, ody bet treble, närmaft utfanten, bildas af trefantiga, inåt ajrunbabe fläfar. Mellan 2:bra ody 3:Dle bans bet finnes en twergående rnb af fwarta punfter, fom motirara öfre flouns od) omgifuras lywarbera of en fôrre lifusgut frets.

Qarven, fom är furart meb ett af [må hyita punfter bes

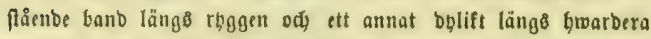
fiban, har föltiga tornar, af bwilfa 6 fla $i$ twerraber på be 2:ne forfla froppringarne, 4 fi̊ $i$ forfant på fifta, ơ 7 i terrraber på lywarbera af be iffrige; ben mederfta af besfa feo. nare tornar fair no̊got framom be s̈friga. Näft గifa froppss ringen bar ännu en torne, fom fair mibt emot ben mederfa od) frrar bafom benne. Stroppen är unbertill grå meo röba fulbafar. Längben $1 \frac{1}{4}$ t. Larben, fom trăffa på Scabiosa 


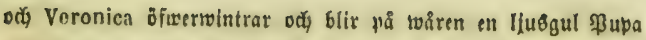
med fmarta punfter of́ ftrect.

\section{IIökblomster-Wärjäirilen (Nolitea dolia W. V.)}

Alis supra fulvo nigroque reticulalis; posticis subtus fasciis tribus favo-albidis, nigro maculatis marginatisque, of duabus fulvis; posterioro nigro punctata. L. al. exp. 40-44 millim.

WBingarne of wan nåtformigt röbgula od fimarta; be bafre uns

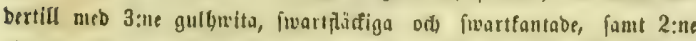
röbgula tuerbanto, of tyrilfa feonure bet baferfa är fiwartłuntterabt. 2. c. wingip. $15 / 8-13 / 16$ t.

Sjn. Pap. delia IV. V. 179. Hülon. Pap. 1. 2. f. 7. 8. TexI. 6. 5. Malir. E. S. III. 1. 2:31. 779. (var. Cinxix). Militzen Ialm. Pan. Sv. 76. 4. Zett. Ins. Iapp. 900. 4. Pap. cinxia De Geer I. 1. 141. 1. 1. . . 13-18. Ochs. Schin. I. 1. 27. IV. 13. God. ot Dup. I.epid. 1. 73. pl. 4. quart. f. 1. II-S. Test, I. S5, t. 56. I. 269. var, Pap. pilosellae Esp. Schm. I. 1. 25. suppl. 1. 1 2. p. 3.2. M. phabe Gosl. Lepid. I. pl. 4. quint. f. 3. (Pap. cinxia Lin. F. Sv. 280, 1063 . non cerle ad hane speciem appertinet, quamquam loc milii verisimile videtur).

Bar. b. Alis supra fulvis maculis nigris basalibus marginalilusque; posticis parto interioro fulvis nigro maculatis, parle posteriore flaro-albidis macula nigra inter costam 2 et 3 , fascia tenuissima fulva, nigro-maculatit, transversa, media, et sorio nigra linearum punclorumque ad marginem.

Wingarme ofwan röbgula med furarta fläđar trib tafen od) i utfanten; be Gafre unbertill till illb:e bälften röbgula

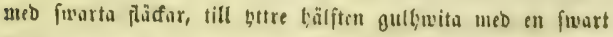
สhä ex emellan 2-3 tringnerben, ett imalt robgult, ftwarts fläfigt tererband oimer niden och en rab fivarta freff od puntter läng utfanten.

Syn. Felitaa fulla Dalm. Pap. Sv. 77. 8. socundum specimon originarium in Museo Pajhullii adsorvalum. Pap. fulla Quens. Act. Lolm. 1791. 279. a. 1. 
Eiärilen förefommer på torra äng8bacfar od betesfălt i föbra o(4) meblerfta Grerige, ber ben tillbörex be allmånnare arterne. 3

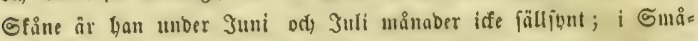

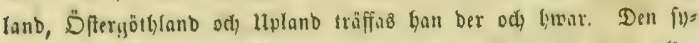

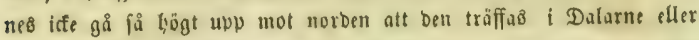

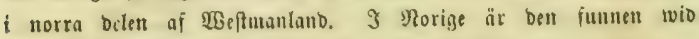
(chriftania (Gisbfe). Bar. b. är męfet iällfunt.

Irtbeffr. 2(ntemnerne bruna, fywitringlabe. SRlubfan ofitan brun, $i$ fränxe fanten och $i$ ipesfen röbgul, unbertill röbgul ods hivite agtig. Balperne brvita, $i$ fpeten röbgula, unbertill meo gri ody bruna bår. Şuimut, rlyggffölo od abdomen ofman fiwarta med röbgul flobb, fon på be tweme förftuämube froppsbelarne à längft. 2(6bomen unbertill finvitagrig. Bröftet grålubet. Eösterne röbgula. Sgannenछ analborfte röbgul.

5aame: WBingarne ofivan röbgula med firarta tvingnerver od) talrifa fmarta, af llâfar beltånde twerbanb, fả att ben jwarta färgen lift ett nät intager rringarnes bela pta. Rüngs

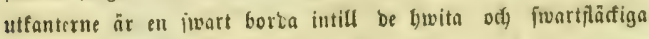
franfarme. Bafningarne bajua uti anbra raben (irån utfonten räfnabt) af be röbgula läafarne fwarta punfter, fon lifna ocel= ler. Ünoertill äro franitvingarne röbgula meb uågra fî fwarta

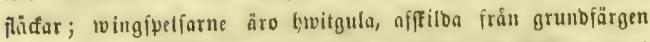
genom en fwart mågformig linea, funt hafwa innanfor benna i främre fanten en fläc af famma färg; längs utfanten går en rab fwarta, fmå punfter. Safwingarne hafma unbertill 3:ne b)itgula, fmartbegränfabe och fwartpunfterabe, trågige twerband, famt 2:ne anbra röbgula; neml. ett breitgult med fwarta punfter wib bajen, bernäf ett röbgult; åter ett lnvigult öfwer mibten, lywilfet långe̊åt år tecfnoot meb fwats päfar ; närmaft Detta ett röbgult, jom $i$ miden lyar en rab juarta punfter, מotfwarande ofre fibans ; fatligen wio utfanten ett fowitgult, fom befair af fammanţängande, halimänformige flärfar méb hwarbera en pwort punts. Ghalbürnet är bmitgult. 
Sounut Ylfnat bannell, men har ben fwarta färgtefullingen på mingarnes öfre fiba merenbels gröfre ode mera fammantly. tanbe, flunbom fi fammanflytumbe, att grunbfärgen fbnes fwart.

Sarven, Gnilfen lyar tornar, fåfom föregåmbe arl8, är fwart meb blågnita, af punfter leftånde ringat oufrinß lebs

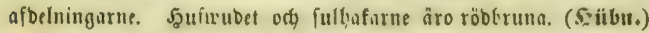
Den lejwer vå llierarcium pilosella, Vorosica od. Plantago, famt öfwernintrar. Pupan bar vrangegula taggar od punfter.

\section{Crodblads-Ratfjäilen (Molita atlalia Esp.)}

Alis supra nigro-fuscis fasciis maculisquo fulvis; pusticis sublus fasciis tribus flavis I. flavo-allidis, impunctalis, fasciisqguo tribus fulvis; posterioro arcuhus nigris. L. al. oxp. $37-40$ millim.

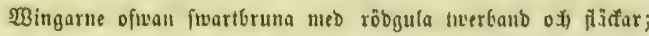
be bafre unbertill med 3:ne liıళgula cller butitagtige, oflärade twer.

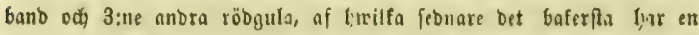
rab furarta bågar. \&. e. rwingip. $1 \frac{9}{16}-1 \frac{5}{8}$ t.

Ș̣n. Pap. athatia Esp. Schm, 1. 1. 47. cont. 23. 1. 1. a. b p. 377. P. malurna Esp. t. 30. Supł!. 6. 1. 2. p. 325. var. Nabr. E. S. III. I. 6'54. 787. Ilübn. Pap. 1. 4. 17. 18. Pap. alhalia Freyer Beitr. 11. 9. p. 4. 1. 49. Melitea Ochs. Schom. I. 1. 44. IV. 14. et 108. Treitsch. X. 1. 7. Ialm. Pap. Sv. 76. 6. Zett. Ins. Lapp. 900. 5. Godart et Daponch. Lepid. I. p. 78. pl. 4 quart. f. 2. pl. 4 tert. f. 6. II-S. Text, I. p. 23.

Bar, b. Alis posticis subtus faseiis nigro inductis; forma minor. I. al. expl. $32-35$ millim.

Bufreingarnes therband unbertill nuggot flötanbe $i$ firart; minbre form. \&. e. mingip. $1 \frac{B / 16}{16}-1 \frac{7 / 16}{\text { t. }}$

Sy̆. Pap. parthenie Borkh, Schm. I. "i3. ".. II. 194. 5. Helliza Italm. Pap. Sx. 77. 9. Ochs. Schm. I. 1. 48. God. ot Dup. Lepid. II. pl. 9, f. 7. 8. II-S. t. 30. 1. 136. 137? P. athalia llibu. I'ap. I. 4. 19. 20. 12. apheca llïbn. 1. 147. 1. 783. 739. M. alhalia II-S. t. 57. f. 270-27\%. Yett. Ins. Lapp. 900. 5. var, b. 
Bar. c. Alis posticis infra basi nigro-maculatis, fascia modia lineaque marginali flavis.

Bufiringarne unbertill wio bajen fwartianatiga mé ett gult twerbanb öfwer mibten od) en linea af fumma färg längo utfanten.

Svn. Melitea her/ha Dalm. Pap. Sv. 77. 7. Zntt. Ins, Lapp. 900. 6. Papilio fuerestl. Act. Iloltm. 1791. 280.8 . 1. 10. f. 9. 10. (secundum specimen originarium in Museo Schonhorsi adservatum).

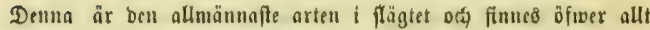

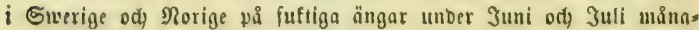
ber. Bar. b., fom af flera författare anję fäfom en egen art, träfs fas, eburu fparfiumt, både i fötra od) norra Siwerige tillfamman med ben wanliga formen. Bar. c. är beremot ganffa fällunt, men bar

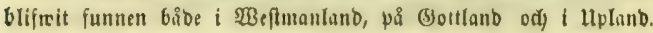

21rtbeffr. 2Intennerne oftwan mörforuna, unbertill buitagtiga eller röbgulagtigu, ofraft otboligt guritringlabe. Bulperne breit=

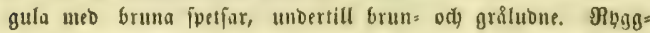
földen med mörfbrun lubb. 2(6oonen ofman fisart meb firarta bår, unoertill huvitgulagtig. Bröftet grått. Fötterne röbgula. J̧annens analborf̣te röbgul.

Till fäigtef̛ningen $p a ̊$ tringurnes öfre fiba varierar benna fläril betyoligr, få att finappt twenne exemplar af hunbrabe ảro Luwaranora fullfomligt lifa. Wingarne äro ofwan ljufare eller mörfare furartbruma med flera eller färre röbgula fläfar oda i

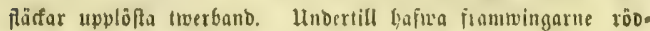
gull fäıg, belo̊ med tublig, bels meb otbolig fmart tecfuing, bes

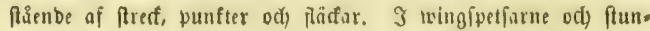

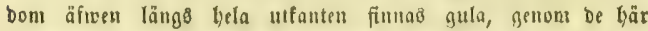
furarta nerberne ftilba lliffar, af hyilfa be fom följa fanten äro fegelformiga oḑ inåt fwartbegränjabe; längs tutterfa fanten löpa 2:ne furarta linier, af fwilfa ben inore är trågionig od mer eller minbre thblig. Bafiningarnes tubre fion, font år minft unberfaftad föränbritgar, upplages af 3:me ljuşgula od 
lifa många röbgula twertianb, fircilfa alla fafna fwarta punfter

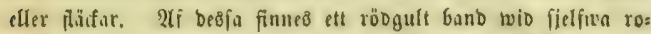
ten, bernäh stt lfusgult eller lymitagtigt, beftäenbe of $3-4$ meb

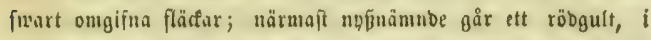
hırilfet funn on lywituglig, fwart begränjab flä ; berefter öfwer biffen åter ett ljuళిgult eller bwitagtigt, wågigt, inåt od) utåt begränfabt meb (mart od) oftapt genom en fwart länge̊gående linea belabt $i$ tuvenne olifa Gäliter; bernáft ett röbgult, $i$ Guril. fet fimnas en rab fwarta halibigar, och flutligen att ljuegult af inåt afrunbabe, fmart begränfabe fläctur, öfner bwilfa, nảrmaft utfanien, löpa twenne parallela fwarta linier. Eranfarne äro hnitagtigt gula med fwarta flädar.

23ar. b. är betbligt minore med fmalare odis mera långs fläfta framuingar, men föröfrigt till färgtcfuningen alloelcs lif föregående, utom beri, att unbre fibanỏ röbguln tmerbanto åro mörfare, öfweroragne meb fwart, få att be ftöta $i$ brunt.

3ax. c. WBingarne ofwan mörforuna med ett af ftồre röbgula fäcfar beffåcnbe tuerbano nära utfanten od flunbom ned en od) annan röbgul flä $i$ biffen. Framuingarne unber. till röbgula mé twenne olifilora fwarta fläfar $i$ biffialtet (fiuns bou besutom meb en eller amnan minbre), famt thert öfwer

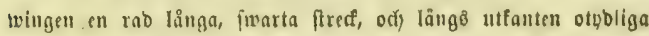
thiangelformiga fläfar of jamma färg. SBafıingarne unbertill röbgula med fhra jwarta fläcfar wib bafert; of wer mibsen ett twerband af liuft offragula flüfar, glvilfa på inbre od bitre fiban bronränjas of ett brebare fwart firef́; bafom betsa banb är twingen fivartbruแ med en rab halfmånformiga, tö̌tgula flăfar od) $i$ utfanten en rörgul linea. Sofwingarne aro unbeifapabe föränbringar till unbre floans färglefning. Cå är ฤ̧08 nåsra exemplar besiła wingas bttre bälft frwitgul nico en matt od oft)blig röbgul frimma odi) längs utfanten fimmes anthoning till ett fwartagtigt twerftred.

garven till ben egentiga M. athalia Esp. är jwart med twenue raber lywita punfter på qwarie fropposing faut tornat 
fåiou hos föregaienbe. Sarben till warleteten b fall hafwa be liwita punfterne fuapt märfbara ody enl rab blefgula päcfar

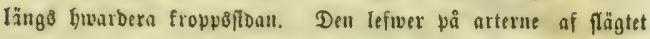
l'lanlago od Valeriana famt öfuerwintrax. \$upau är till förra formen gråbruแ med fwart teđ̛ning yå twingfitoorna od fwatta fant röba junftraber ya abbominalregionen, men tilf den febnare formen ffall ben wara affegrå med 2:me raber roffärs gabe punfter pio abbominalregionen. (Od)i.)

\section{Kohvete-Wïtfjärilen (Molitæa dictynna Esp.).}

Alis supra nigris fulvo maculatis; posticis infra fasciis tribus allidis, impunctalis, of tolidem fulvo-brunnois, postoriore arcubus nigris, maculis llavis punctisque nigris adjacentibus. L. al. exp. $34-37$ millim.

DSingarue ofwan fiwarta med röogula flücar; be bafte unber. till med 3:ne fyritagtiga twerband utan fwarta punfter, ods lifa mångn anbra röblruma, af fywilfa jebnare bet baferfta yar en rab fwarta balfGågar, intill bwilfa ligga gula fläfar od fwarta puntter. l. c. wing 1 p. $1 \frac{3}{8}-1 \frac{1}{16}$ t.

Sy̆. Pap. dictynna Esp. Schm. 1. t. 58. suppl. 24. f. 2. a. b. t. 91 , cont. 41 . f. 1. p. 180 . Kabi, E. S. III. 1. 253. 785. Melitxa 0ehs. Schm. I. 1. 12. IV. 14. Dalm. Pap. Sv. 76. 5. God. ol Dup. Lepid. I. 80 pl. 4. f. 3. pl. 4 quint. f. 4. II-S. Text. I. 24. Pap. corithalia. lliibn. t. 3. f. 15. 16. Texi. 7. 10.

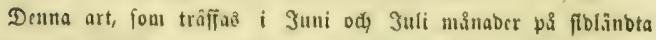
fogbangar, tillför endaft föbra Emerige ody Notige. 3 Sfalue är ben p.? flera ftällen allmän, fajom wid Trolle Rjunglh, (58̊phult odh

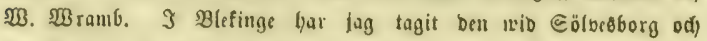

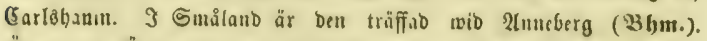

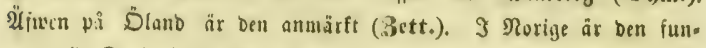
nen $n$ io Sanbefiorb (Bicbfe).

Urtbeffr. 2Intennerne mörfbruna od britringlabe. Slubban of man brun, ofta $i$ bafre funten lywit, unbertill och $i$ fpetfen röbs

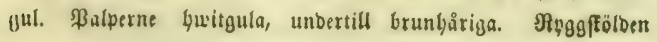


röborunt Gåig. ఇ(6bomen ofwan fwart meb forta bår af

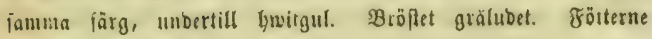
röbgula.

Saaute od boun: Willgarme ofiwan fwarta meb flexe eller färre röbgula, minbre fläfar; be Gafe hụiwa fällan mer ăแ 3:ne raber fådane flüfar. Unbertill ăro be främre mürft rö̀. gula ucb fivarta flisfar; längs urfanten löpa twenne fwarta farect, ai liwilfa bet inbre år mer eller minbre wågigt od utgör 乌)ttre gränien för en rab trefantiga limbgula, inåt lifalebes fwart

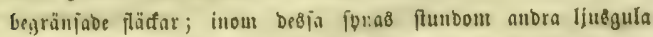
Flüfar, fom for riwinna mot wingens inbre fant. Bafwingarne Lafua på unore floan $3: n e$ linsogula eller juarare lywitagtiga od tifa många brungula fanb, af lymilta be forra fafna fiwarta puntıer. Desia band yafiwa föllambe orbning: närmaft bajen ett bumgult, $i$ brvilfet twid fielima rotloben finnes en buitagtig fläc ; berefter ett bywitagtigt, fom beftůr a olifftora fuartbegräns jabe fläfor ; fron siter ett brungult med en bueit, af flwart oms

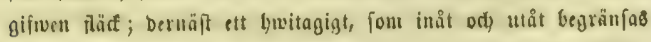
of en fwart linea, ory belas genom ett fivart langeggående, afbrus set fircof $i$ tweme büliter, aj hyilfa ben inbre, jom är minft, pfta bar gulagtigare ninfruffning än ben butre; nårntuft betta nicr ett brungult, jom bar en rab fwarta lyalibingat, guarbera omflutande en lfuลgul faăf iemte en fwart punft; gtterít finnes

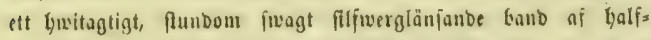

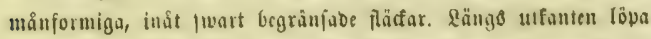
twenne parallela firarta linier, gwiltas mellanum är röbgult. Rerverne ăro på bufwingatues undre floa jwarta. Franfarne Lusita med jwarta fläđar.

थ̈fiwen benna fiăril är unberfaftad sariationer, hocf ef till ben grab fom forregiende. Fliffarne på ivingasnes öfie floa äro

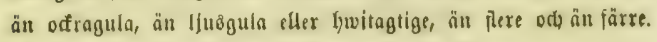
Stumbom äro bafuingarme nåfan enfärgabt fwarta.

Larven är röbagtigt violett med lfusblå punfter ods 3 :ne

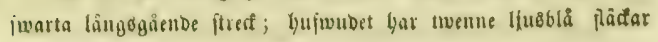


ody tornarne, foum bafwa famma beffaffentyet, fom hoo närmaft föregåenoe, äro $i$ fpetiarne firarta. Den lefwer på Nelampyrum nemorosum od Valeriana officinalis.

\section{Slägtet: Argynnis. FABr. et Adcr.}

Antemm longiorns, gracilos. Clava brevis, ovalis I. suborbiculata, in speciminibus oxsiccatis excavata, compressa.

Palpi capite longiores, adscendentes, apicibus divergentihus, triarticulati. Articulus medius maximus, tumidus, infra hirsutissimus, ad latera foro nudus; ultimus minutissimus (basali brevior), compressissimus, nudus apico acuminato.

\section{Dcull nudi.}

Pedes antici breves, feminæ minus, maris tamen magis pilosi, tarsibus inarticulalis; cotori ut in gonore præcodento, armati of instructi; femora infra aut nuda aut pilosa; articuli tarsorum ut in genere præcodente, rarius ut in sequenle.

Alæ subdentatæ. Collula posticarum costa spuria tenuissima, anticarum sat distincta, clausa.

Costæ alarum posticarum $9:$ i 4 o trunco communi superiori (prima ranulum refexum I. rectum in lobum emittons), 5-7 o trunco inferioro, 8-9 o basi egredientes. Cellula discoidalis inter costam 4 at 5 costa tenuissima Iransversa clausa. Cosiæ alarum anticarum 9-10: 1 o hasi, 2-4 o trunco communi inleriori, 5 o costa transversa cellulx, 6-8 o trunco communi superiori (7 quadriamosa), 9 o basi egredientos; sunt tamon costæ alirum 10, costa 6-9 - trunco suporiori ( 7 triramosa) et 10 o basi egrodiuntur. Cellula discoidalis costa transversa intor costan $4-5$ sat distincta clausa.

2ntsuncrue långa, fina. Slubban fort, oval cller något runbab, 5̧0 torfade eremiplar urljolfad odj jaumantrbet. 
Palperue längre ån bufwubet, uppåtfliganbe, mé bibergeranbe fpets far, treledabe: meblerfta leben ftörti, uppolaft, nebtill ganffa lus Den, på fiborne uäftan 6ar; fifta leben gauffa liten (fortare ẩn basleoen), ganffa fammantrydut, nafen ody tilipetiab.

\section{S̈goucn nafna.}

Fötterue: be fräuire forta, hos Gonan glefare, yos Gannen längre lubne, meto olebabe tarfer; be ôfriga fåfom Gos föregåenbe flägte bewäpnabe; li̊ren antingen nafna eflet lubna; tarjernes leber jü.

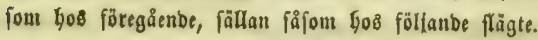

MBitgaruc fmåtanbabe. De batres biffält flutet genom en fin, falft twernerb, be fräntres genom en temligen tyblig.

Rerverte på Gafwingarne $9: 1-4$ utgåenbe frân ọ̈̂̉e neruftam. men (ben 1:fĩa utjändonbe åt rotloben eн Gafåtbỏjo eller rät gren), 5-7 från nebre neruftammen, 8-9 från bajen. Dift: fältet flutet genom en fin twernerv emellan 4-5 ivinguerwerme. Franıwingarneతి nerver 9-10: 1 utgåenbe från bafen, 2-4

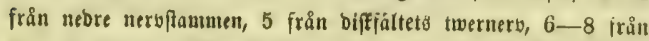
ofre nervftammen (7:be forgrenig), 9 från bajen; nen äro ner. verne 10, få utgå 6:te-9:be nerberne från öfre nexuftammen (7 tregrenig) od ben 10:be från bajen. Difffältet flutet genour en ganfea tọblig twernerb emellan 4-5 toingnerwerne.

Detta artrifa flägte bat rerprefentanter båbe $i$ Sfanbinaviens fobligafte ody norbligafte belar. Några arter utbreba fig ofwer ljela Galfön, anbra äro beremot inffräntta till twisfa prosinjer. Gomliga älffa fogarne med beฮิfas torra marfer, anbra beremrot äıgarne od

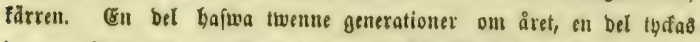
beremot blott ega en. Larven, fom är befläbd nteb bårba, greniga, i fer raber fiăllo tornar, bland hivilfa merenbels twenne ftörre od, lăngre finnas̊ på förfta froppsoringen, lefwer på lägre bicorblibona

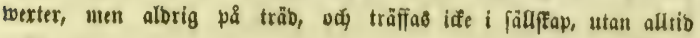
enorig. 


\section{Subdiv. Âtminstone medlersta fotparets lär un- dertill tiitt ludue*).}

1) Framvingarnes nerver 9.

a) Fürsta nervens pá bakvingarne gren rät.

\section{Gvartringlade Perlemorfjarilen}

\section{(Argynnis aphirapo Hübu.)}

Alis supra fulvis niyro maculatis; posticis infra fulvis fasciis favo-albidis, interdun argonteis, macula media fascie discoidalis linea niłra transversa divisa, serieque marginali punctorium ocellarium.

L. al. exp. $37-40$ millim.

W3ingarme ofwan röbgula med fwarta fläfar; be fafre unbertia röbgula med lyritgula, fantom perlsmorfärgabe twerband, af livilfa Det medlerftą mederita fläa år på tweren belab genom ett fwart ftred; ; nära utfanten en rab occll:lifa punfter. \&. \&. wimgip. $1 \% \frac{16}{16} / 8$ t.

Słn. Pap. aphirape llibu. Рap. t. 5.. โ. 23-25. t. 163. 1. 811. Argymuis Ochs. Schm. I. 1. 52. IV. 14 \& 109. Treitsch. X. 1.8. Dalm. P'ap. Sv, 69.6. Zett.

-) Tabula subdirisionum hujus generis:

A. Femora pedun saltem inter-mediorum infra dense birsuta:

1. Costœ alarum anticarum 9 :

a) Ramulus cosla primo ala-) Aphirape. Selene. Freja. Polaris. rum poslicarum rectus: $\{$ Pales, Frigga. Thore.

b) Ramulus cosin primn ala-) rum posticarum arcuatus
reflexus:

2. Coso alarum anticarum 10; Ino. ramulus coslø primø alarum
posticarum rectus: $\left\{\begin{array}{l}\text { Ino. } \\ \text { Lathonio. }\end{array}\right.$

B. Femora pedum iofra nuda; costo
alarum 10; ramulus costo prime
alarum postiearum reflexug: $\left\{\begin{array}{l}\text { Nibbe. } \\ \text { Adippe. } \\ \text { Aglaja. } \\ \text { Paphia. }\end{array}\right.$ 
Ins. Lapp. 895. 6. Godl. ot Dup, Lepid. II. pl. 9. f. 3. 4. II-S. Text. 1. 34. Pap. thomyris llerbst. Schm. t. 270 . 1. 6. 7 .

Bar. b. $\left\{\begin{array}{l}\text { Fascia media alarum posticarum ut etiam maculis ba- } \\ \text { seos infra argentuis. }\end{array}\right.$

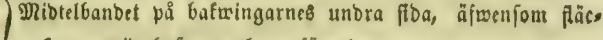
farne wib bafen perlemorfärgabe.

Syn. Pap. ossianus llerbst. Schm. 1. 270. f. 4. 5. Ilübu. t. 146. f. 734. 73\%. Argymis fiod, of Dup. Lepid. Suppl. 1. 129. pl. XX. 1. 5. 6. Boisl. Icon. 94. pl. 19. f. 1-3. $\|-\mathrm{S}$. Text. 1. 34. t. 67. . 322. 323.

Mrten tillyör egentligen męrtrafterna $t$ norra belarne af wår G.lfë, ber båba barieteterme träffas till famman med lywaranbra ody äro temligen allmünna. Dơ forrefommer fiürifen ni̊gon gång âfmen i mederfa Swerige. Ga har ben blifuet funnen wid Porberg $i$ Beft.

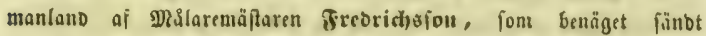
crcmplar till påfeende. $\Im$ norben är ben träffad wio \&ufịele or

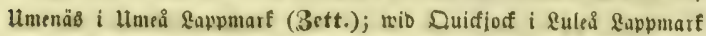

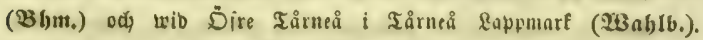

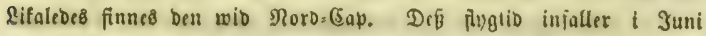
ody Suli mainaber. Utom Sfanbinavien finnes ben i alla norbliga tän.

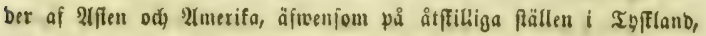

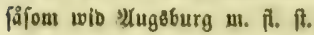

Artbefer. 2(ntennerne ljuฮ์bruna, ojwan ated brcba finita ringar, unbertill röbgula, Slubban brun $i$ fpetfen od) unbertill róbgul. \$alperne rỏbgula, undertill brunbåriga. SPbBgftöben med röb. gulagtigt bruna båx. 2tbdouten ofman firart, fortbårig, un. bertill röbgul. Bröftet brungrått. Fötterne röbgula, meb Grun. agtiga lår.

Şautue: Mingarne ofwan röbgula, wib bajen mer eller minbre bruma, neb fwarta fred, twerlinier ofly flüar, be jeb. nare nâra utfanterne runbabe. Liffanterne, fom äro fwarta, átfôljas af en rab jurarta trianglar, fom buabera innefluta en

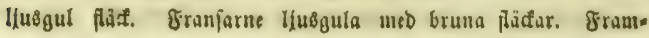


wingarne unbertif röbgula, men Yăngs utfanten faint $i$ wing.

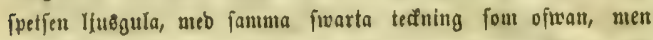
ben är laăr finare od utfantens trianglar fammanbänga la̛e meb fwaranbra. Bafwingarne brungula meb $3-4$ olteftora,

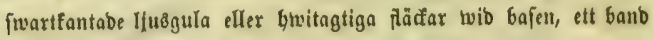
af bylifa pläfar p̈fwer biffen ody en rab trefantiga altio perlermor. färgabe, inåt od utåt fwartbegrånjabe fläcfar läng ut utfanten. Mibtelbanbet, fom är furartbegränfabt, yar ben frora mibteliäđđen, hıเvilfen merenbels är perlentorfärgao, belab genom en fneb. gåenbe fwart linea. (Emellan famma banb od) utfanten finnes en rab af Iluşgula eller fiwitagtiga, ocell=lifa punfter omgifne meb en

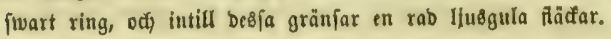

Sgonan lifuar fyannen, neen Giar grundfärgen oftwan mexa Dởragul ợ utfanterne lịiare.

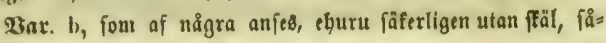
fom egen art, ftiller fig från nu beffrifua beri, att bet furarta wib uningfafen ofwan är minbre utbrebt; fäđarne och mibtelGanbet på Gafwingurnes unbre foba perlemorglänfanbe ợ att

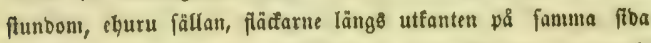
af Gafwingarne äro ef triangulära, utan fmarare rectangelformiga.

Qarusu fort od thod, rjuft filfwergrå meb ganffa forta tornar od utan halstornax. Fुufwubet brungrått. Wib byarie torne finnes på raggen en fwart ftrcá, od intwib benna en liu= fare linea. Nebanför luftyåten fynes en ywit floolinea. Wus

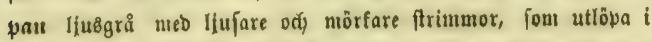

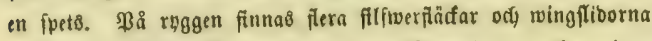
äro flatft inböfoa. (Freber.) \&arben of fwersintrar od unbers gå fin metamorplyos i Maá månab föllanbe året.

\section{Brunfackiga Perlemorfjarilen}

(Argynnis solone Nabr.).

Alis supra fulvis nigro maculatis; posticis infra flavis brunneo maculatis, fasciis brunneo-fulvis of flavis, his plus minus 
argentois, macula intermedia fasciæ mediæ argentea, indivisa, serioçue marginali punctorum nigrorum cœecs. L. al. exp. 40 - 43 millim.

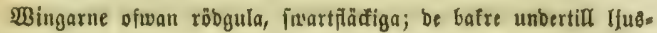

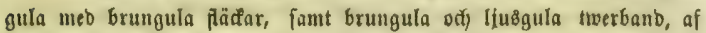
quvilfa be feonare äro mer efler minbre flfwerglänjanbe; mibtelban=

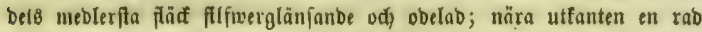
fwarta enfärgabe punfter. ฉ. e. twingip. $1 \frac{5}{8}-1 \frac{3}{4}$ t.

Syn. Pap. selene Fabr. E. S. III. 1. 147. 451. Mübn. Pap. 1. 5. f. 26. 27. ot figg. 732. 736. 738. Argymnis Ochs. Schm. I. 1. 55. IV. 14. 109. Treitsch. X. 1. 8. Dalm. Pap. Sv. 69. 7. Zett. Ins. Lapp. 896. 7. Godart ot Duponch. Lepid. 1. 64. pl. 4. tert. f. 4. II-S. t. 33. f. 150. 151. t. 34. f. 152. 153. (var.).

Var. b. Alis supra fulvis macula rotundala costali propo basin limboque exteriori nigris; hic serie punctorum fulvorum; anticis infra fulvis macula ocellari strigaque brovi ad marginem anticum nigris; posticis infra flavis costis punctoque basali nigris, maculis brunneis in parto posteriori.

\$3ingarme ofwan röbgula neb en rumbab fwart fantiäđ nåta Gajen; hattre fanten ặ çammı fârg, men berjente röbgult fläđ̛̆ंg; be fräntre unbertill röbgula meb en fwart

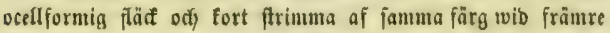
fanten; be bafre unbertifl blefgula med fwarta nerver od en bylif păå twio bajen, famt bruna fläđar $i$ twingarnes buttre byălft.

Syn. Arg, selene var. b. Zett. I. c.

Var, c. Alis supra fere ut in priore; anticis posticisque infra serie macularum brunnearum, his flayo alboque lineatis, costis punetoque nigris.

Wingarne ฟå öfre flban näftan fon hos föregåenbe; un: bertill meb en rab fruna flädar; be bafre beputtom linierabe mes bwitt od blefgult, faut forfebbe meb en jwart puntł odi nerber of famma färg.

Sỵn. Arg. selene var, c. Zett. I, c. 
Var. d. Alis supra nigro-fuscis maculis fulvis, ad marginom posteriorem seriatim disposilis; anticis supra disco intoriori macula fulva, rotundata, costali, propo basin nigra; omnibus infra ut in var. c pictis, sed maculis brunneis longiludinalibus, venas cingentibus.

Bingarne ofman fwurtbruma med röbgula fläđar, fom Filoa en turerrad nära utfanten; be fränre ofwan meb en runbab rôbgul fäa wio infanten, f̧wilfen närmare bajen ä frrart; alla wingarne unbertill färgabe fåfon hos bar. c,

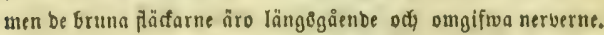

Syn. Arg selene var. d. Zett. I. c. Pap. rinaldus llerbst. t. 271. f. 1. 2. 4.

Var. e. Alis supra nigro-fuscis maculis rarioribus navis; anticis infra fulvo nigroquo maculatis; posticis infra fulvo fuscoque variegatis, fasciis 2 serieque punctorum marginalium flavis.

Wुingarne ofiwan fwartbruna meb färre röbgula fläfơ ; be främre unbertilf fwart od röbgult fläđige; be bafre på famma fiba marmorerabe med röbgult od brunt od ega 2 röbgula smerbanio faut $\mid$ utfanten fläcfar af famma färg.

Syn. Arg. selene var. o. Zett. I. c. Pap. marphisa llerbst. เ. 270 . 1. 8.9.

Var. f. Alis ut in priore, sed posticis supra nigro-fuscis unicoloribus.

BBingarne fåfom hos foregåenbe, men be bafre ofwan enfärgabt jwartbruna.

Syn. Arg. selene var. I. Zett. I. c.

Var. g. Alis supra sericeo-brunneis, unicoloribus; posticis maculis 3-4 minutis fulvis ad angulum ani ; infra omnibus ut in var. 0.

Wingarne ofwan flifesbruna, enfärgabe; be bafre meb

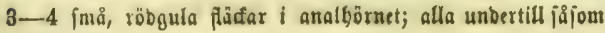
hos yar. $\theta$.

Syn. Arg, selene var. g. Zett. I. c. 
Denna fläril, ell af be allmännafte inont flägtet, träffas (ito minftone $i$ S. Siverige i twenuc generntioner om äret - pil alla fanta ängar öfwer allt på wå̉ Galfö, be norbligafte trafterne ef unban= tagne. Från Tromso bar ben blifwit bemföro of 2101. Eiljebora i

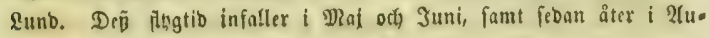
gufti. Gebnare generationen är ef fi̊ talrif fom förra. Baricteterme,

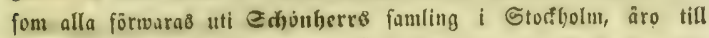
förfta belen från Sappland od) D̈ftertiotten. Doof förefomuer, en eller annan af bem äfwen $i$ föbra ody mederfita Swerige. De äro lifwäl alla fällphnta.

Artbeffr. 2ntennerme 6runa, otb̧bligt biritringlabe, Rlubban brun, i fpetfen röbgul. Balperne på floorne britagtiga, ofwan od) unbersill röbgulagtiga, lubna. Rnggffötoen luben af röD= gult. 2tboonen ofman fwart meb röbgula piffellasir, unbertill ḩwitagtig. Bröftet brungrått, lubet. Fōtterme lłu\&bruma grå. pubrabe.

Syanue od, boun lifna biwatanbra, ment bariera betsoligt till färgtef́ningen. Den wanliga formen har wingarme of wan

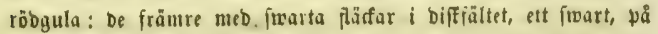
flera fisullen aforutet twerband ofwer mibten frin framfanten till infanten, od en rab runba, fwarta pläfar närmare utfan. ten, Gwilfen âr fwart med flöre eller minbre, runbabe eller tretantige röbgula fläđar; De Gafre, fom wio bajen äro fwarta imed en röbgul ring, omgifmanbe en fwart runbad flä bafwa ojuer mibten ett jwart, afbrutet tuerband, bernäp-en

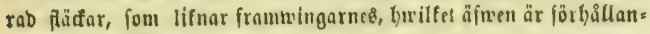
bet mis utfanten. Linbertill äro frammingarne blefare röbgula neeb finare (mart tefning od) gulagtige witugfuesfar, lywareft finnaอి nůgra röboruta flücfar. Baftwingarne äro ljue̊gula meb ett af 3-4 aplingt:runbabe, hiwitgula cller lifuegula, mer eller minbre fllfwerfärgabe fläfur beftůnbe banb twid bafen, ett annat bhlift af 9 oliffora, uerendels fmart begrinnfabe, Aäcfar öfwer biffen, ody ett treble af 7 , inåt juarthegränjabe, trefuntige fläcs far I uffanten, hwilfa oftafł alla s̈ro falimerglänfanbe. EMuélan 
bafens och bifens lifuęgula twerbanb går ett roftbrunt banb, i Giwars mibt finnes en ftörre, runbab, frwart fäđ, ơ eneflan bis ffens od utfontens twerbanb finnas $2-3$ ftora, obegränfabe xảbbruna fläfơr, äfwenfon en rab frwarta, enfärgabe puntter,

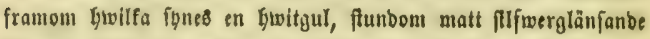
flamma, fom på mibten är affruten. Mibtelfanbets meblerfta

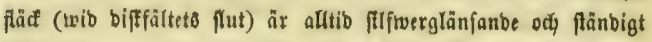
obelab (utan thblig fwart fuebftrect). Franjarne äro Yjuฮgula meb bruna fẳăar.

Baten beftrifwes fwart meb morfgrd frimmor, och rofts gula, gleft fwartlyåriga tornar, af liwilfa be twå förfta äro rigtabe utåt. ß̊̊ förfta fropperingen funnas twenne buita punfter på lytwarte fiba. Dep uäringsiplantor äro arterne of fläglet Viola. अupan är gulbrun, meb fwarta, fläđơ od taggar. (Treitssh.)

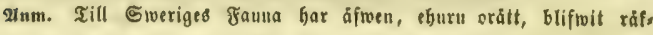
nab en art, fom fórefommer $i$ simlanb od aunorftábes, neml.:

\section{Argymis amothusia (FABn.)}

Alis fulvis nigro-maculatis; posticis infra variegatis, fasciis duabus dentatis, altera flava, altera rosea, punctis 5-6 posticis ocellatis, et uno busali nigris.

2Ringarne röbgula, fwartfảafiga; be bafte unbertill marmorerabe meb twenue taubabe twerbaub, bet ena gult, bet anbra rofenfärgabr, faunt 5-6 froarta, ocellformiga bunfter nára utfanten en bylif wib bafeu.

Syn. Pap. amathusia Fabr. E. S. III. 1. 255. 791. ARGYNNis OCIS. 1. 1. 75. DalM. Pap. St. 71. 9. ZETT. lns. Lapp. 897. 9. Gob. et Dup. Lepid. II. 65. pl. 8. f. 5. 6. H-S. Text. 1. 35. Honn. Pap. Text. II. 14. Pap. diana Hobn, t. 10. f. 51-54. PAP, tithania HaBN. t. 9. f. 47, 48.

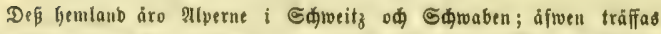

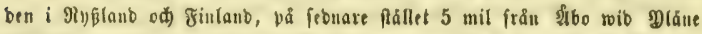
Mugåro, men bar åun iafe filtills blifwet funnen uti Groerige eller Morige.

\section{Frejas Perlemorfjäiril (Argynnis ireja Thbg.)}

Alis supra fulvis basi maculisque nigris; posticis infra forruginois albo fuscoque variis, fascia transrorsa inedia lunulisque 
marginalibus flavo-albidis, puncto discoidali nigro, albo circumcincto, seriequo postica punctorum nigrorum. L. al. exp. $35-10$ millim.

Wingarne ofwan röbgula meb fwarta flăthat och bas?; be bafre unbertill röbbruna, marmorerabe med britt och frunt, meb ett bivits gult, tinergående miblelbanto od) i utfanten fläđar af famma färg, fant i biffältet on fwart, af howitt omgifwen punft, äfwenfom en rab fwarta punfter öfwer wingens bafre bälft. \&. e. Ivingfp. $17 / 16-1 \% / 8$ t.

Syn. Pap. freja Thunb. Diss. vol. III. p. 49. t. 5 , f. 14. Quens. Act. Holm. 1791. p. 276, 6. t. 10. f. 5. 6. Ilïbn. Pap. t. 10. f. 54. 55. t. 11. f. 56. t. 154. f. 771. 772. Argymis 0chs. Schm. I. 1. 78. iv. 15. Dalm. Pap. Sv. 71. 10. Zett. Ins. Lapp. 897. 10. God. et Dup. Lopid. Suppl. I. 122. pl. 19, f. 6. 7 . Boist. Icon. p. 102. pl. 19. f. 4. 5. II-S. Text. I. 36.

Derma art tillyör egentligen fisllylatauerne i Rappmarferne, ber ben ifbunerbet finnes talrif omfring Quiđfod, men ben nebftiger äfo wen på låglanbet, fåfom $\mathrm{i}$ Fimmarferne. Uti Nortbotten är ben

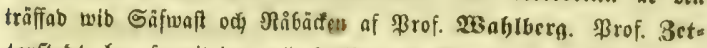
teritebt, har funnit ben wib Rndfele ody Stenfele $i$ Uncå \&apputart. \$rof. Bobemen bar beurfört ben från Dowre. Flygtiden infaller i Suni ody Suli månaber.

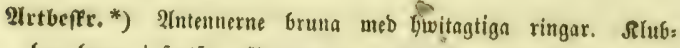
Gan brun, $t$ fpetīen röbgulagtig. \$alperna gulagtiga, unbertill

") Descr. Antenns fusco annulis albidis. Clava fusca, apice fulrido. Palpi fulvi, infra fulvo pilosi apice fuscescente. Caput et thorax fulrohirsutissimus. Abdomen supra nigrum, breve fulro-pilosum, infra flavoalbidum 1. fulvidum. Peclus hirsulum, fulrum. Pedes dilute fulvi.

Mas. Alo supra fulvw, ad basin nigræ, præsertim postica. Macula nigrw ordinariæ rudiores quam in speciebus præcedentibus. Alæ anticœ infra ochracex, ita maculato in disco ut in parte superiore, spicibus tamen flavidis et lineolis Iongitudinalibus ejusdem coloris in margine intra costa8. Ala postice infra ferruginem maculis 3-4 flavo-albidis basalibus, et inacula altera in cellula discoidali puacto minuto aigro instructa. Per 
röbgult Kårign, $i$ ipetien brunagtiga. Şufwub od rhgaftölb flurft lubna af röbgutt. It (coonen ofisun fmart, forthârig af rỏbgult; undertill lywitgulngtio eller foòtande 1 töbgult. Bröftet röbgult lubet. รötterne ljuf röbgula.

Sranue: Wingarne ofwan röbgula, wid Gafen frrarta, lywilfet lismnerbet ầ förbållanbet pii be bafrs, ber bet fiwarta fträder fig stôfwer nibten. De wanliga fiwarła tedningar äro gröfre ăl hos föregienbe arter. Inbertill äro frammingarne ofragula meb famma fmarta teffning $i$ biffen fom på ö fre fiban, men wingfperfarne äro ljuegula od) längo utfanten fi̊ cmellan nerverne en rab l|usgula längsftref. De Gafre äro unbertifl rôbfruna med 2-3 hrvita flütar wio bafen ord $i$ biffältet en nnnan, minore, af fanmma färg, 1 mibten förfido med en fwart punft. Śfwer mibten går ett hnitgult, meb röbbrant ber od̆ Givar of merpubrabt, af olifformiga läffar bilbabt merbanb, fom utît ody inait begränfas meo en rad fmartbruna, pilformiga fläfar, byriffa mānó fuetiarne mot buaranbra. Detta Ganbo medierfta ilăd (mio biffiältets glut) är på tweren belab genont en fwart frrect. Emellan farima banto od) utfanten funce en huritagtig, i gult fötande ftrimma, bafom huvilfen funneళ en rab fwarta, fumbon omärfliga punfter. Qäng utfanten går en

medium Iascia flave-albida, line inde brunnen adspersa, e maculis difforunibus compositn, utrinque agittis nigro-fuscis, apiripus sibi invicem spectantibus, marginata, se extendit. Macula madia (ad apicem cellulo discoidalis) hujus fascico lisea transsersa nigra diviso. Inter fascian jam memoratam et masginem exteriorem altera est fascia indeterninata medio ioterrupta, albida, in flavedinem vertens, post quan series punctorum nigrorum, plus minus distinctorum, observatur. In ipsa margitue macula flaru-abida, ovala I. trigonæ, intus angulis fuscis marginato, adsunt. Fiumbrim flavide, fusco-maculatæ.

Femina mari est similis, sed maculus flaras supra in margine geret.

Hab. in Lapponia, ad Quickjock Irequens; ad Safrast et Rảbacken, Lycksele et Stensele, Nuonioniska, Karcsuando, et Juchasjarvi rarius. 
rab binitgula, inåt meb brunn winflar begränfabe, ovala eller

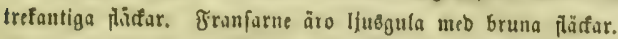
flăd๋ar.

Syouan lifnar bannen, men bar ofuran $i$ utfanten liasgula

\section{Nordislka Perlemorfjäilen}

- (Argynnis pólaris Dolsd.).

Alis supra fulvis maculis nigris; posticis infra fusco-ferrugineis maculis fasciisque transversis ot lunutis T-formibus marginalibus ut etiam puncto discoidali lacteis, soriegue postica punctorum nigrorum. L. al. exp. 40-45 millim.

WSingarme ofwan rōrgula meb fwarta fläfar; be bafre unbertill

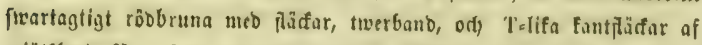

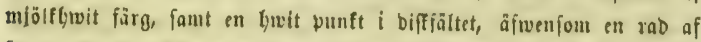
fwarta punfter ôfiver wingarnes bafre hălft. $13 / 10$ t.

Syn. Arg. polaris Boisd. Ind. meth. I, 15. Icon, 104. pl. 20. 1. 1. 2. God. ot Dup. Lepid. Suppl. I. 125. pl. 20. f. 1-3. Zett. Ins. Lapp. 89\%. 11. H-S. Text. I. 32.

Denna art, fom mḩdit lifnar föregaienbe, träffas rib famma

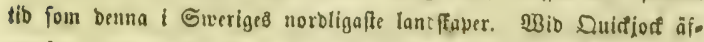
twenfon i Finmatfen är ben tagen af $\mathfrak{B r o f}$. 3 ahlber ${ }^{*}$ ).

Irtbefr. 2ntenter, palper, qufwoto, reggffölb ody abbomen lifna till färgen famma partier loos föregiende art. Fötterne od bröptet äro brunagtiga, bet febnare furft lubet.

Syameno wingar ofiran lifut röbgula, wio bafen af framwingarne fỏga brunagtiga; houns beremot môrft röbgula, rnib bafen fiwarragtiga. Fỏr ôfrigt ftililes arten från föregående genom föllanbe făıneteđen, bämtabe af bufwingarneछิ unbre fiba:

-) Ilab. in terris Scandinaricis maxime borealibus cum pracedento, at ad Quickjock ot in Finmarkia. 


\section{Arg. polaris.}

Bafmingarnies unbre foba fwartag. tigt röbbrun från bafen wiba stöfwer mibten.

Mib bajen af bafwittgarne区8 unbre fiba 3-4 imtïre miolfhbita fีăđåar.

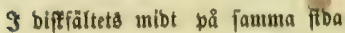
af batwingarme finnes en fowit

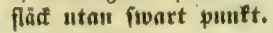

D̈iwer biffen ett twerbanb af mitolffonita, med frunt ber ods bwar öfimerpubrabe fläfar, htwils fet inåt od utåt Segränía $\mathrm{cn}=$ Daft af ben fmartagtigt robs= brutıa grunbfärgen.

Samma Ganbo mibtelfläå belab på tweren of elt fill, brü ftreff.

গुib flutet of ben sruna grunbs färgen går twers ôfwer wingen

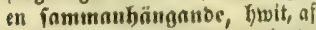

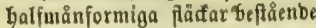
frimıı, intill Grvilfen ben fwarta punfrraben befinner fig.

3 utfanten fintres en rab af mlölts Gwita flăđar, fou bafwa liffbet nted boffinfiven $T$ od int begräufas af ben fwartagtigt rö๖bruแล gruแbfärgen, fom bär intager utfanter ody frams om fläcorne bildar allora tres fantiga flảifar *).

\section{Arg. freja.}

Bafiningarnesి unbre fiba röbbrun utöfwer mibten.

Wib bajen af Gafwingarme区 unbre fida 3-4 fiöre bwitgula ศึีäår.

3 biffältete mibt på fanuma fiba of baftwingarne finnes en hiwit=

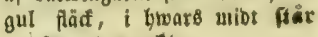
en fwart pustt.

Dfwer biften ett twerbanb af hivit. gula meb röborunt ber od brar

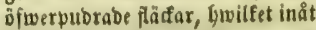
od) utåt begränfas of en rab fตartbruแa, pilformiga fläcs far, fom wảnba petfarne mot Gwaranora.

Samma banbชి mibtelfăcã belab på - tweren af en grof, fourt fredo

Wुib flutet aỉ ben rōbbruna grunb. färgen går twers öfwer wingen en afbruteu fowitgulagtig ftrim: ma, från hwilfen punftraben befinnes frilo genom orunbs

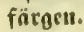

\$ utfanten finres en rab af bwits agtiga đảçar, fom åro till for men ovala ellex trefantiga, od inåt begräujos af fwarta vinflar, bwilfa tboligen ftilta fị från ben rỏbbruna grunb. färgen.

-) Descr. Antenno, palpi, caput, thorax et abdomen ut in priorc. Pedes el pectus fusco-forruginci; Loe dense hirsutum. 


\title{
5. Gulntickiga Perlemorfjárilen
}

\author{
(Argynnis pales W. V.).
}

Alis supra fulvis nigro maculatis; posticis infra rufo-brunneis maculis basalibus fasciaque media maculari flavis, of maculis marginalibus, macula obliçuo trigona fascix medix punctoque discoidali minuto argenteis, ot macula flava intra-marginali, supra seriem punctorum fuscorum adscondento. L. al. exp. $35-48$ millim.

Maris alse sunt in parte superiore dilute fulræ, anticoe ad basin haud fuscescentes; feminœ tamen obscure fulva, ad basin nigrescentes. Proterea hac species parto alsrum posticarum inferiore a priore, ut sequitur, facillime dignoscitur :

\section{Arg, polaris.}

Alis posticis infra fusco-ferrugineis a basi longe supra medium.

Ad basin 3-4 maculis minoribus ef lacteis.

In medio cellula discoidalis macula alba impunctata.

Per medium fascia transversa maculata laclea, hine inde brunneo adspersa, utrinque fundo fuscoferrugineo marginata.

Macula media fasciæ inemoratæ linea tenuissima, fusca, transrerse divisa, Ad finem lundi fusco-ferruginei striga transversa lactea, a maculis lunatis composita, continua, ad quam series punclorum nigrorum adest.

Ad marginem exteriorem inaculis lacteis, litteram T. mentientibus, intus fundo fusco-ferrugineo, angulos formante, marginatis.
Arg. freja.

Alis postcis infra ferrugineis longe supra medium.

Ad basin 3-4 maculis majoribus et flaro-albidis.

lo medio celluloo discoidalis macula flavo-slbida puncto nigro.

Per medium fascia transversa maculata flavo-albida, hine inde brunneo adspersa, utrinque sagittis nigro-fuscis, apicibus sibi invicem vertentibus, narginota.

Macula media fascio memorata livea rudi, nigra, transverse divisa.

Ad finem fundi ferruginei striga transversa interrupta, flacida, a qua series punctorum nigrorum coloro fundi sejuogitur.

Ad marginem exteriorem maculis oratis I. trigonis, flavo-albidis, intus angulis fuscis marginatis. 
WBingarne ofisan robogula, freatfliatign; be bafre unbertill rŏb.

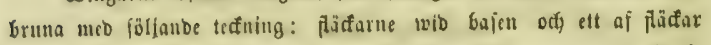

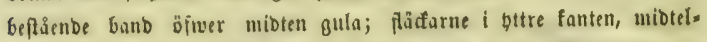
banbets fuedt triangutära fläc od en liten punft $t$ biffältet flfiwer. gynita; wib utfantent en gul flüf, foum uppltiger offwer en rab bruna puntter. \&. e. twingip. $1 \frac{7}{16}-1 \frac{15 / 16}{\text { t. }}$.

Syn. Pap. pales W. V. p. 177. Vabr. E. S. III. 1. 257. 797. llibn. Pap. t. 7. †. 34. 35. t. 121. f. 617. 618. Argynnis Ochs. Schim. I. 1. 63. IV. 15. \& 110. Treitsch. X, 1. 11. Dalm. Pap. Sv. 72. 11. Zett. Ins. Lapp. 897. 12. God. of Dup. Lepid. II. 68. pl. 9. I. 1.2. Pap. pales Thunb. Diss. vol. III. 48, cum varr.

Var, b. Alis latioribus rolundatis; posticis infra ut in prioro, sod macula intramarginali tlava ad soriom punctorum fuscorum adsiendento.

Wingarne brebare, afrunbabe; be Gafre unbertial fåfom Gos föregiende, wen med en gul flä 1 utfanten, fom ens Daft upplfiger till en rab bruna puritier.

Syn. Pap. arsilache Esp. Schm, I. t. 56. cont. 6. f. 45. llibu. Pap. 1. 7. 1. 36. 37. Aryyunis Treitsch. Schm. X. 1. 12. II-S. Toxt 1. 36. 1. 55. 1. 259-262. Arg. carichlea God. ot Dup. Lepid. Suppl, I. $344 . \mathrm{pl}$. 48. ค. 1. 2. (oxcl. synon.)? Arg. pales var. Dalm. et Zett. I. c.

Obs. Varr. Isis ot Napoea llïbu. in Scandinavia non occurrunt, qrantum novimus.

Denna art tillyör egentligen fogafärren od bergetojoerne $i$

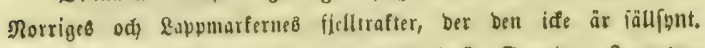
Mera fällan träffas ben $i$ jöbra od) medlerfta Giverige. S norben förefomma varieteterne tilljamman med hmaranbra, men $i$ fooligare provinfer of mår balfö träffas enbaft var. b. Denne feonare är funnen wio 2Inneberg (3̧)ın.) od Şamneda (3ett.) i Gmåland;

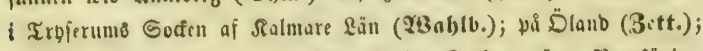
wio egendomen Ringftud $i \mathfrak{B}$. Enelu Soden nira Rorrföping

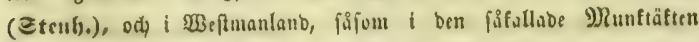

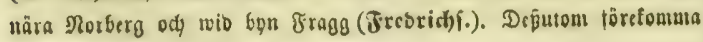


Gåba varieteterne i Gerna Goden m. fl. ft. i Dalarne (Bh)m.); $i$

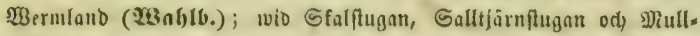

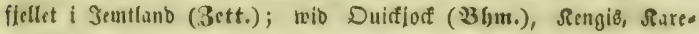
fundo od) Eydjele (3ett.) i Rappmarferne, fant i D̈perbalen, (3)

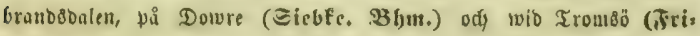

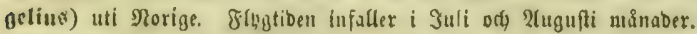

Artbeffr. Ansemuerne oiwan gulbruna, unbertill byitagtiga. Rlubban brun, i fpelín röbgul. Balverne gulagtiga, ofran ods neban röbgula, lubna. Rpggfä́bent röbgult fuben. 2tobomen ofman mco röbgula, forta, gleja laar, unbertila gulagtio meb tãtare lubob. Bröftet röbgult, luobet. Fötterne röbgula.

Sanme: $\mathfrak{B}$ Bingarne ofman rỏbgula med fwart, af röbgult bifnerpubrab bas, ods famma fwartu tedningar fom bos fant= flägtingarne; lifuäl äro be bär betubligt finare, få att bet öfIner biffen gaindne, mågiga ort) på flera ftällen aforutna twer. bandet åt fmalare, ifynuerbet pá Gafuingarne. Unbertill ảro be robgula wingarne iörfedoa med mįcet finare, flunbom fnappt märfGar fwart tefning; wingfpetfarne l’huถgula med en eller fiere röboruna, utåt något greniga flüfar. Baftringarne, fimilfa $i$ främse förnet äro farpt minflade, bafma på unbre fiban röboruแ grunbjärg. Nårmaf̧ bajen finnas $3-4$ aplingt-runs

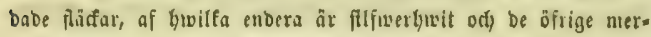
enbels gula. $\Im$ bef berpafülfande twerbanbet af grunofärgen finneß $i$ bifffäItıt en itten, funbom omärflig, filînerlywit punft. D̈fmer mibten går ett ai olifformiga pläfur fammanjatt twer. banb, hnwilfet är gult; ben breba, fucbt triangelformilya lasa,

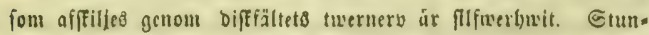
Dom äro odja ben gुtterfte ody innerfte af besfa plidar filfurer=

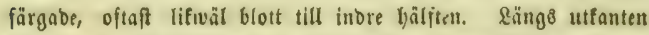
finnas 7 fiffuerfärgabe, inåt brunfantabe, trubbiga hisfar ods cmellan besfa jant mibtelbanbet finnes en rab fruma punfter, ftunbom omgifne fuarabera med en gul ring o(t) förfeobe meb pupill, od :nom besija funes en flifwerbwit, pá mibten aforuten

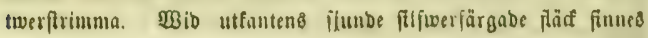




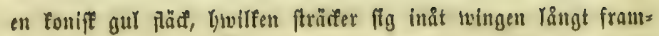
on bell fruna punftraben, fa att bell ena bitföranbe punften

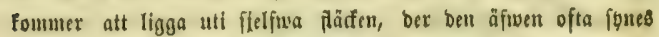
otb̧blig. Franjarne äro gula meb bruna läçar.

Soura lifuar bannen, neen bar byftrare grunbfärg od, ben fmarta tecfningen på ivingarnes unbre fiba gröfre.

23ar. b. Sifnar föregåenbe, men har brebare ody mera af= rumbabe twingar famt är fiürre. Dé̉ jwarta tef́ning ofwan

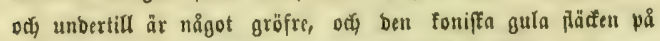

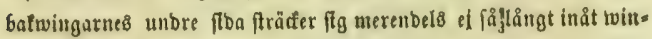
gen, fon bos föreguenbe, utan flutar oftuft innan ben binner ben mörfbruna punftraben. Rifwäl ăro besija furafieter of conftanta, utan öfwergångar finnąి, få att man ofta befimuer fị $i$ willtå. bighet till Gwilfenbera ett exfyallet exemplar ftall föras, Gwax= före man el fan med asftilliga författare anfe benna warietet for egen art.

\section{Snrven*) - - - - -}

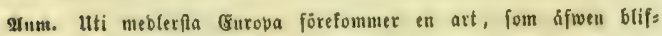

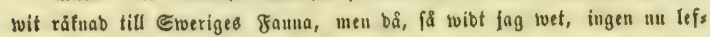
wanbe fwenf (ร) ntumolog jar fị någon local inom wånt lanb befant, ber

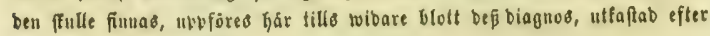
exemplar från Tyftano:

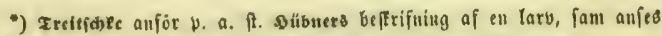

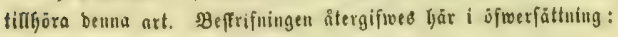

"Den farns af Ediffermlitler $i$ ofra Difterrife vå blaben af Viola montana. Dȩ́ lufwub war hierfformigt, utan märfbata tornar, fortgirigt

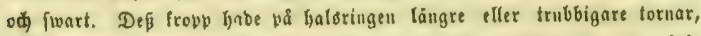

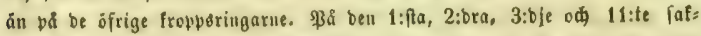
nabes fibutornarne. Dell war glänfanbe fammetofwart, blott ryggtoriarne ftodo alla på citrongula fláctar od woro áfwen tifl beras mibt af fauma

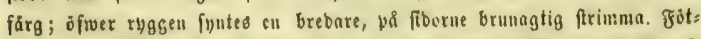

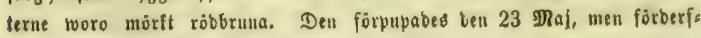
wabes meban ben dunu war mjut." 


\section{Araynule dia LiN,")}

Alis supra fulvis, nigro maculatis; posticis infra fusco-purpureis, basi flavo argenteoque maculatis fascia obsoleta maculisque marginalibus argenteis, puncto flavo, nipro circumcincto in diseo, serieque postica punctom rum fuscorum subocellatorum.

MBingarue ofwas röbqula, fmartfáciga: be bafre unberfifl brunt bur, burfärgabe, meb gula od filfmerfärgabe flả far wib bafelt, eft of bolight fulf:

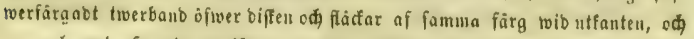
en gul meb fwart ongifmen bunft $i$ biffen, famt nára utfanten en rab bruna, till en bel ocellstifa punfter.

Syn. Pap. dia LIN. S. N. II. 725, 207. FAnR, E, S. III. 1. 255, 792. Argrnnis OGhs, Schim. I. 4, 61. God, el Dop. Lepid. 1. p. 66. pl. \& secund. f. 1. pl. 4. quint. f. 1. DALv. Pap. Sv. 72. 12. Zetr. Ios. Lapp. 898. 13. H-S. Text. 1. 32.

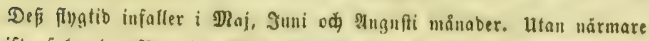

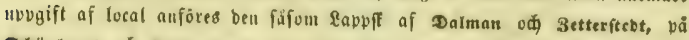

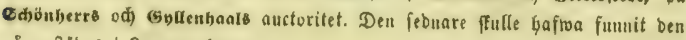
någonfẩbe i Raupmarlerne.

\section{Nriggas Perlemorfjiril (Argynnis Irigga Thbg.)}

Alis supra fulvis basi maculisque distinctioribus nigris; posticis infra basi brunneis, postice testacoo-purpurascentibus : fascia modia macularum lulvarum albarumquo, sorie punctorum fuscorum, linoa maculari submarginali ojusdom coloris, mar-

-) Intra limites Scandinarim non occurrit quantum milii inotuit. A peregrinatoribus hujus sovi in Lapponia non reperts. In collectionibus Entomologorum Suecoram et in Museo Academia Scinent. Reg. Holm. specimina quidem plura exstant, indicatum tamen non est si suecana nec ne. - Angynis carichlea IIsRest - (0chs. Sichm. IV. 114. DALa. P. S. 72. 13. Zert. Ins. Lapp. 898. 14. Ang. arclica Zert. I. c. 899. 18. Arg. Boisduvali Soms. Borso. Icon. t. 20. f. ö. 6.) in Lapponia Scandinavica non est reperta. A Dom. Quenser tantum collects, ut videtur in Lapp. Rossica J. in Finlandia. Nec in Finmarkia Norvegio occurrit. Specimen, e Groenlandia ortum, a Celeberr. ZeTtensteot descriplum et a mo in museo ejus ditissimo collatum, est Ang. carichlea ef cum Ang. Boisduvali sat bene convevit, quare hoe nomen in symonymia citare non Lositavi. 
gineque immaculato; macula ovata discoidali fasciæ linea nigra obliqua subdivisa. L. al. exp. 40-43 millim.

Wingarme ofwan röbgula med fwart fas od fwarta, förre

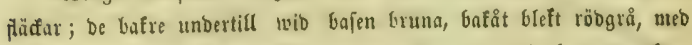

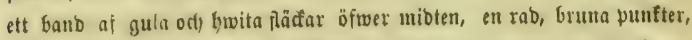
od) närmare utfanten en af fläfar beftåenbe linin meb fauma färg; fielfwa utfanten ofläafab od) meblerfta fläcen i miotelbanbet äggfor. mig, belab genom ell jucb fwart ftreff. \&. e. wingip. $1 \frac{5}{8}-1 \frac{3}{4}$ t.

Syn. Pap. Prigga Thby. Diss. vol. III. 47. llïbn. Pap. t. 9. ศ. 49. 50. Irgyunis 0ehs. Schm, 1. 1. 74. IV. 15. Dalm. Pap, Sv. 73. 14. Kett. Ins. Lapp. 898, 15. God. et Dup. Lepid. Suppl. I. 120. pl. 19. 1. 3-5. Boist. Icon. 100.4 . pl. 19, f. 6. 7. II-S. T'ext. I. 32.

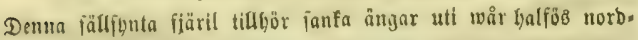

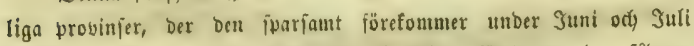
månaber. Utan att fign foọt upy på fiellen, förebrager ben făbana ftälen ber wibet ody allora pilfuffar wexa. Lti Dre Soden $i$ Da. larne är ben funnen af \$rof. \$3obeman, hyvifen äfwen träffat ben twio Quifjoct $i$ Ruleå sappmurf. WBio D̈fre Torneå är ben funnen

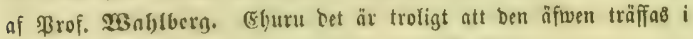
Rorffa Sinunarfen, fänner jag ef betta med jäferfyet.

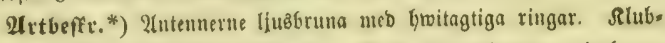
Gan brum, i ipetfen gulagtig. \$alperne gulagtige meb bruna

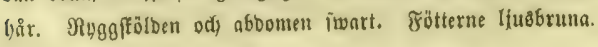

-) Descr. Antenne fuser albido annulalo. Clava fusca apice fulrido. Palpi fulvidi pilis fuscis. Thorax ut et abdomen fuscus. Pedes dilute fusci.

Mas. Ala supra lulre, ad basin nigro-fusew, maculis nigris ut in congeneribus; prope marpinesn exteriorcm immaculatum adest linea transvera nigra. Ala anticse infra pallide fulsw, ita maculata ut in parte superiore, sed upicibus ferrugineo-maculatis. Als posticx a basi usque ad medium fersuginex macula alba ad basin fasciaque macularia, arcuata, in medio. Macula fascia, margini antici proxima, alba, cum macula basali fere connexa, quo fit ul area triangularis ferruginea includatur; ina. cula discoidalis fascite (ad opicem arcole locata) ovata, alba, linea transversa nigra oblique divisa; inaculo onnes relique fasciæ bujus fulvæ, 


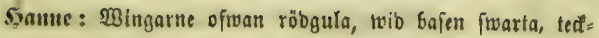
nabe med fwarta fääfac fåjom lyos famflàgtingarne; nära uts fanten, brwilfen för öfrigt är ofläđab, finnes en faumanbăn. ganbe fiwart twerlinia. Framtvingarme unbertill bleft röbgula meb famma fwarta tedoning jom på öfre fiban, men meb bruns Tlästiga fpetīar. Bafwingarue från baịen, utổiwer mibten, röb. Gruna meb en lywit päấ wio bafen ody ett af flădar beftåenbe, bågbölbt banb öjwer mibten. Tiverbanbeta, wib framfanten the

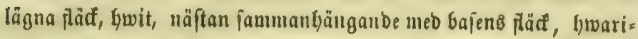
genom ett triangelformigt, röbbrunt fält inneflutę ; troerbanbets

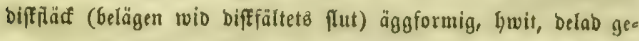
nom en twergåenbe, fHeb, jwartagtig linia; twerbandets ofriga fläcfar röbgula, merendels of fwerpubrabe med mörfare färg. $\Im$ biffẫltet finnes ofta en îwart punft, fom onıgifmes af en sul cirfel. Bafningarmes yttre bălft bleft röbgrå med en bwitây.

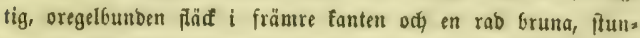
bons ocellormiga punfter. Mära bttre fanten finnes en tmer. gåenbe, mörffrun linia, iamman[att af aflånga flāđar. B)ttre fanten olädab (utan de Iwanliga lymita fläfarne). Franjarne hwitagtiga meb bruna fiäçar.

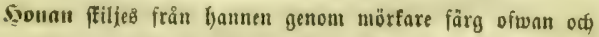
renare twerband på bafningarnes unbre fiba.

colore obscuro plerumque adspersæ. In areola discoidali punctum niprum, flave circumeinctum, sxpe adest. Pars posterior alarum posticarum est testaceo-purpurascens inacula irregulari albida ad marginem anticum, serieque punctorum fuscorum, interdum subocellatorum. Prope marginetn exteriorem est linea fusca, transversa, ex maculis oblongs composita. Margo exterior immaculatus (absque maculis ordinariis albis). Fimbrim albo, uaculis fuscis.

Femina a inare dilfert colore supra obscuriore et fascia puriore in parte inferiore alarum posticarum.

Hab. ad Ore Dalecarliæ; ad Quickjock et Ofre Torneå Lapponiæ mensibus Junio et Julio, rarius. 


\section{\%. Grikantade Perlemorfjärilen}

\section{(Argynnis thoro llülon.)}

Alis supra fulvis basi maculisque majoribus nigris; posticis intra basi fulvo-fistacois fascia maculari modia flava, ad oxtimum ferragineis màculis fusco-forrugineis subocellatis maculisque marginalibus roseo-albescontibus; macula oblonga discoidali fasciæ linea obscuriore transversa tenui in maculas duas inæquales divisa. L. al. exp. 45-50 millim.

Wingarme ofwan röbgula meb fwart ba8 ofi fiotre fwarka

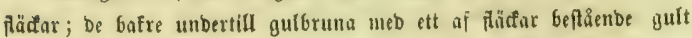

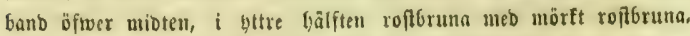

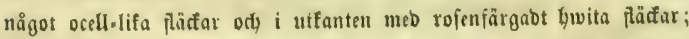
medlerfta fläden $i$ mibtelbanbet aflång od) belab genom en mörf od) fin twerlinea $i$ twenne olifftora fläfar. \&. e. twingip. $1 \frac{18 / 16}{16}$ t.

Syn. Pap. thore llülun. Pap. t. 111. f. 571-573. Irgynis 0chs, Schm. I. 1. 71. anm. IV. 15 et 111. Treitsch. X. 1. 14. Dalm. Pap. Sv, 74, 16. Zett. Ins. Lapp. 899. 17. Boist. Jeon. 96. pl. 20. 1. 3. 4. God. ot Dup. Lepid. Suppl. I. 118. pl. 19. I. 1. 2. II-S. T'อx।. І. 31. t. 30. ศ. 138. 139.

Demna är en af Sfanbinaviens fällyntare fiärilarter ợ tiflyör

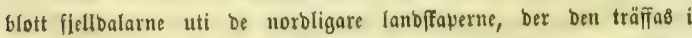

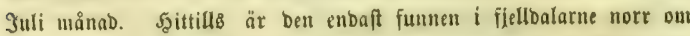
Dowre i Noriģe (336m.) odj uti en Dalfträfuing wid Baliftellet

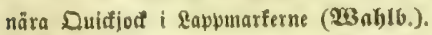

Artbejer. SAntennerme ofwan bruna mob buitagtiga ringar, uns Dertill rôdgulagtiga. Rlubban mörforun mé röbgul fpets. \$al. perne gulngtiga, unbertill nicb röbgula, ofwan meb brunagtiga Går. Siygoffölben jwart med mörft röbgul lubb. 2lbbomen ofwan iwart meb glefa röbgula bår, unbertill gulagtigt grå. Bröptet röbgult, lubet. Fötterne röbgula.

Sonue: Bingarne ofwan röbgula, wib bafen (iibmnerfet De bafre) fwarta med be ivanliga fwarta tediningarne, bivilfa

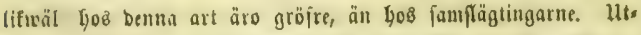

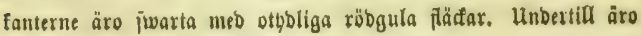


frambingarme $i$ biffen röbgula odj $i$ mingfpetjarme blefgula meb fmärre roftbruma fläfar od) faggningar. För birigt lyafwa de näftan jamma fmarla tefning jom ofwan. Bafmingarme äro wib Gafen gulbruma, utan beftämba, gulagtiga flä̊far; öfwer mibten går ett af fläffar beftående gult twerbanb, jom bafảt bes gränfas af en fwartagtig, tanbab linia; meblerfta lläten af nåmmbe twerbonb är aflång ợ nätntate inbre fanten belab genom ell bojlb, brunagtig, fin linia, frwilfen, äfwen gå̊nbe öfs wer ben närmafte fläđe̊t, utmärfer bifffältet\& twernerv. Dittre bälften af bafwingarne är roftorun meb fölianbe märfbara tedै. ning: $i$ framfunten od $i$ inbre tanten nära avalförnet finnç

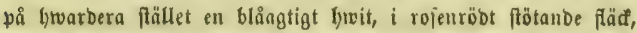

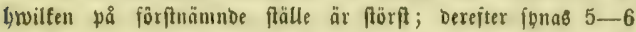
runba, i rab fällba, mörft roftoruna flicá, bnilfa till förre eller minore bel något lifna oceller; 2:bra eller 3 :błe af besfa (från infanten räfnabt) ligga uti cu gulaģtig ftörre flä ; lāng utfanten finneB ett blangtigt l)witt, i rujemöbt pötanbe, af aflinga Färfar bilbabt twerbanb, jom är mer eller minbre tobligt od inåt begränfas af ett roftorunt påctGanb. Franfarme byitgula meb bruna fläfor.

Ş̣onan är ofman ljufare odj unbertill på bafwingarne

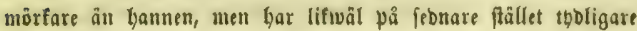
tedning àn benne.

b) Första nervens pả bakvingarne gren krökt, utảtböjd.

\section{Prydliga Perlemorfjirilen}

(Argynnis eupbrosyne Lin).

Alis : upra fulvis nigro maculalis; posticis infra forrugineis maculis basalibus fasciaque media maculari flava; macula basali, macula intermedia fascize indivisa, maculisque marginalibus argentois, serieque marghinali punctorum nigrorum ubocellatorum. L. al. exp. $45-50$ millim. 
W3ingarne of wan röbgula, fwartiăåiga; be batre unbertill xoft:

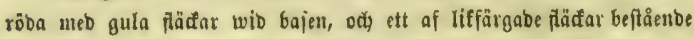
banb อ̈fwer urioten; en fä̛̆ wib bajen, meblerfta flăfen af mibtelbanbet och fläåarne $i$ utfanten filfwerfärgabe; Derfemte nära utfanten en rab fwarta, något ocellformiga puntter. \&. e. wingfp. $1^{13 / 16}-2$ t. Sp̧n. Pap. euphrosyne lin. F. S. n. 1069. Nabr. E. S. 111. 1. 147. 450 . Ilübn. Pap. t. 6. f. 28-30. Argymnis Ochs. Schm. 1. 1. 58. IV. 14. Treitsch. X. 1. 10. Dalm. Pap. Sv. 70. 8. Zett. Ins. Lapp. 896. 8. God. ot Ilup. Lepid. I. 61. pl. 4. f. 1. pl. 4. tert. I. 2. II-S. T'ext. I, 33.

Var. b. $\left\{\begin{array}{l}\text { fulvo-maculatis. } \\ \text { Minore : wingarnes utfanter ofwan fwarta meb röbgula }\end{array}\right.$ ก̣ăḍ̛ar.

Var. c. $\left\{\begin{array}{l}\text { Alis supra nigro-fuscis maculis fulvis. } \\ \text { Wingarme ofwan fmartbruna mé röbgula fläđar. }\end{array}\right.$

Syn. Pap. fingal llerbst. t. 270. 1. 1-3. Arg. Nephele H-S. t. 76, 1. 371, 372.

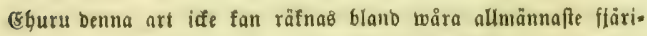
lar, finnes ben boff fpribb öfwer hela wår balfö änba upp $i$ Eapp= marlerne. \$å fanfa ängar $i$ ffogetrufter träffą ben twenue gångex om året. Förfta gången $i$ Maj od) Juni famt anbra gången $i$

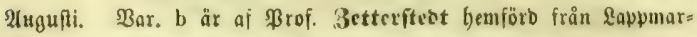
ferne, men förefoumer äfwen i be föbra prosinjerne. Bar. c bar

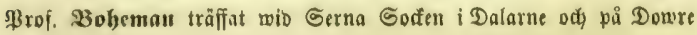
i Norige.

2rtbeffr. 2ntennerne bruna meb buita ringar. Slubban brun, $i$ ipetjen röbgul. ßalperne lịug̊gula, meb röbgula, $i$ fperjen

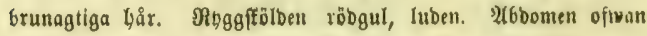
ned röbgula, glefa bår, unbertill gulgrå. SBrôftet gulröbe, lus bet. Fötterne röbgulagtiga.

Syaume: Wingarne ofwan rổbgula med be wanliga fwarta tef̊ningarne; utfanterne fwarta meb en rab fwarta fingemellan ffilba făåar. Frnmuringarne unbertill i bifễen röbgula meb fanıma fwarta 
tečning fom ofwan; wingfpetfarne blefgula meb roftröba flädar. Bafwingarme unbertill roftrôba meb gula fläfar wib bafen oda ett twerbano af på lifa fätt färgabe, olifformiga läafar g̈fwer mibten. Snbre fäa̛fen wib Gajen, mibtelitäfen af twerbanbet, Gwilfen ẩ obelab, ở 7 trefantiga, inåt med roftbrunt begrān. fabe fäcfar i utfanten filfwerfärgabe, glänjanbe. Enrellan mib= telbanbet od) utfanten ät wingens roftröba jurumbfärg belab af gula flammor, of lywilfa en uppftiger från utfanten, en annan går inåt wingen från framfanten odj) åter en amman fro̊n infans ten. ßå nämube bel ai ningen fimes äfwen en rab fwartbruna

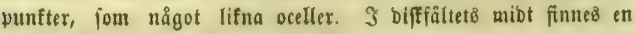
liten, jwart, me’ röbgult omgifwen punft. Franjarne Gwitgula mé bruna fiäcarar.

Sonan lifuar hamuen.

3ar. b. är minbre än föregåenbe ody ftiljer fig från benne Glott beri, att be fworta fä̧arme $i$ utfanterne på mingarnes ofre fiba fantmantyänga finzemellan odj mco utfantens fwarta

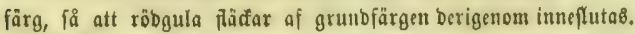

3ax. c. Gar frambingarme ofwan $i$ bifen röbgula uteb orebig, fammanilntanbe fwart teffining. Utfanten är fwartbrun med en rab ljusgula fläcfar. Bafivingarne äro ofwan fwart. bruna meb en rab röbgula fläfar $i$ utfanten od) flefgul infant. Undertill äro be främre från roten, utöfurer mibten, längs infanten fwartbruna, berefter röbgula meb orebig fwart tedning; utfanten odf wingīpetjen fywitgul, ben förta med otyoliga fwår till be wanliga fiwarta winfelpäfarne. SBatwingarne fwartbruna mè röbgula flammor; för öfrigt lif föregåcnoc.

Eatyen är jmart meb fmartbruna tornar ợ twenne jmâ Gunita ftref på lywarie froppsiring, lywilfa bilda en rugglinia. \&uftlålen äro belägne uti en lywitgrå linia. Sulbafarne äro röbbruna. Den dfwerwintrar od) lefwer på arterme af flägtet Viola, fåfom öfrige larber till pertemorffärilarne. \$upan ลำ brungrå, trubbig, ợ har flera flfwerfärgabe funfter tå broffts ody abbominaloregionen. (Ireitich.). 
2) Framvingarnes nerver 10. Första nervens på framvingarne gren răt.

\section{Orangefirgade Perlemorfjärilen}

\section{(Argynnis ino Esp.)}

Alis supra fulvis nigro-maculatis; posticis infra flavis maculis basalibus lasciaque media maculari testaceis, serio punetorum ocellatorum in fascia interrupta rubesconto; macula discoidali fasciæ modiæ linea transvorsa fusca in maculas duas fere requales divisa: L. al. exp. $35-40$ millim.

Wingarne ojwan röbgula, fwarthäafiga; be bafre unoertill gula

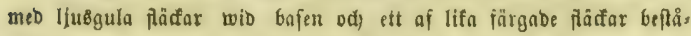
enbe twerband öfwer mibten jamt en rad ocellexifa punfter $i$ en ajs bruten röbagtig binbel; medlerfta fläfen 1 mibtelfanbet genom en twergående brun linia belad $i$ twente uäftan lifa ftora fläđar. \&. e. wingip. $1 \frac{7}{16}-1 \frac{5}{8}$ t.

Syn. Pap. ino Esp. Scbm. 1. 76. c. 26, 1. 1. a. b. p. 125. Irgymuis 0chs. Schm. I. 1. 69. IV. 15. ot 110. Treitsch. X. 1. 14. Dalm. Pap. Sv, 73. 16. Lett. Ins. Lapp. 898. 16. God. ot Dup. Lepid. II. 63. pl. 8. f. 3. 4. II-S. 'Jext. I. 31. Pap. dic/ynna llíbu. Рap. 1.8. f. 40.41.

Denna fiäril är en af̂ be allmännafte arterne inom flägtet ợ förefommer $i$ alla proyinfer, fåmâl ạ Swerige jom Norige, be norb.

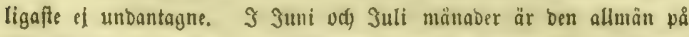

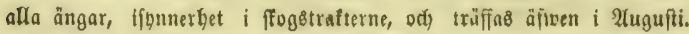
3 meftra od. föbra Europa finnes ben el.

Irtbeffr. 2ntemerne liuft bruna, unbertiff röbgulagtiga. Rlub. Gan fwart, $i$ fpetien röbgul. Balperne blef̣ula meb mörfa

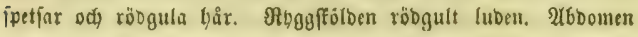
ofwan fwart med forta, glefa röbgula bär, unbertill gulagtigt grå. Bröftet gulagtigt, lubet. Fötterne rỏbgula.

Sanuc: Wingarne ofwan röbgula, meb fwarta tečningar fâjom bos famflägtingarne; utanten brebt fwart meb en rab fwarta, merenbels ftilba fläđar berinnanför. Unbertill airo frame

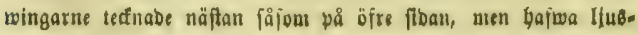


gula fpetfar meb rollfătgabe तỉơar De bafre äro unbertill gula med roftgula päăar wio bafen vdy ett bylift af pärfor

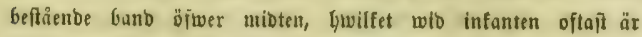
betäcft med mörfa atomer. Wribtelilüden af betta banb är Delad $i$ twenne säpan lifipora gälfter genom en fin, brun linia,

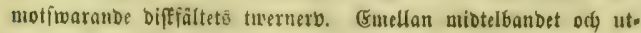
fanten äro wingarne gula meb röblirun ffuggning, bwilfen wa mibten är affruten af grunbjärgen ody uti bivilfen finnes $\mathrm{en}$. rab gula punfter, Jwarbera omgifwen ai in fwart ring. Nära mibtelbanbet finnes $i$ jammia ffuggning ett afbrutet, hwitagtigt, mex eller minbre violett twerbanto, $\Im$ utfanten går en fwart linia off innaniör benna twesme anbra, röboruna. Franfarne bwita meb bruna fäåar.

Sounu lifnar Gannen.

Rarvel har på bwitgul grunb bruna tornar meb fwarta Går, laingftrintmor od enftildo ftreă af famma färg. Ђ马ufwubet ar frunt, hiertformigt, belabt genom en Glefgul frrinma. Den fall lefwa på Urlica urens. \$apau àt gulbrun meb gula ipetjar.

\section{Lilla Perlemorfjäilen (Argynnis latonia Lin.)}

Alis anticis margino exteriori oxciso, supra omnibus fulvis nigro maculatis; posticis infra ochracois maculis magnis, winoribus interjectis argentois serieque postica forruginoa ex ocellis argenteo-pupiliatis, L. al. exp. 40-50 millim.

Framtringarne $i$ bttre fanten utfurna; alla wingarne ofwan ròd. gula, fwartiläafiga; be Gafre unbertill ofragula meo flora od mellanligs ganbe minore flifwerfärgabe fläđar, famt en rab rofloruna oceller med fllfweriärgade pupiller. ‥ e. ivingip. $1^{5} / 8-2 \frac{1}{16}$ \&.

Syn. Pap: latonia lin. F. S. n. 1068 . Fabr. E. S. III. 1. 146. 449. llübn. Pap. t. 11, f. 59, 60, t. 120. I. 613. var. Text. p. 11. 14. Argyunis Ochs. Schm. I. 1. 80. IV. 15. \& 117. Dalm. Pap. Sv. 69. 3. Zett. III. Lapp. 895. 5. God, et Dup. Lepid. I. 59. pl. 3. f. 3. pl. 4. tert. 1. 1. II-S. Text. I. 29. 
Denna wad̛̃ art ăr på åtrar ođ̆ ängar $i$ föbra Swerige

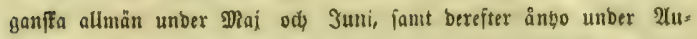
gufti od September månaber. Sebnare generationen fyues wara talrifare än ben förfta. I norbligare lanbffap af meblerfta Swerige

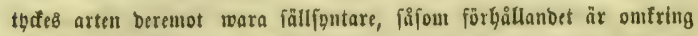
Norberg $i$ Weftmanlanb (Freorich) F.), od) träffas ben inom egentliga

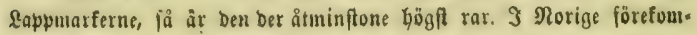
mer ben med fäferbet änba till Tronbbjeur (Sicble).

Artbeffr. Antennerme bruna. SRtubban brun, i ipetien röbs gul. ßalperne ofman röbgula, unbertill hwita, lubna. 5̧ufwub odh ruggftölb meb röbgul lubo. 216bomen ofman fwart med glefa röbgula bår, unbertill hwitgul. Bröftet brvitlubet. Fötterne gulagtigt Gwita.

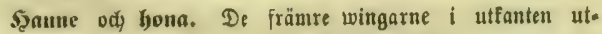
ffurna; be bafre $i$ fanuma fant jutåtandabe. 2ala mingarne of wan röbgula, wib roten mörfare, meb firöbba rumba od̆ aflåแga

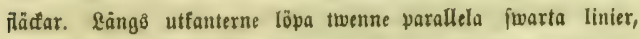
innanför hwilfa $i$ frammingarnes fpetfar ftå på jwart grunb twerne fmärre, merenbels bleft röbgula Flä far. Unbertill äro frantwingarne röbgula, ted̛nabe med fmarta flã đar fåjom på ¿fre fiban, men $i$ wingfpetjarme fimes en roftgul Ramma, lywars uti fem förre, filfwerfärgabe fläfar äro belägna, temte twenne noôrfa flfwerpupillerabe punfter. SBafwingarne äto unbertill flammigt odragula med mörfare utfanter, längs hwitfa finneø̊

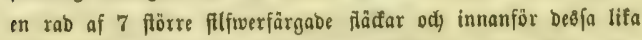

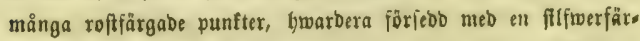
gab vupia. Fỏr őfrigt finnå fpribba bå Gwarbern bafwingen 9 ftörre filfwerfärgabe flăfar, af lywilfa den ftörfte, fom är oval, är belägen $i$ mingenz miot. Emellan beฮิja finnas fiera eller fârxe minbre af famma fârg.

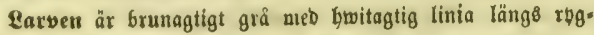
gen, troenne brungula fibolinier od̆ fegutentfanter af jamma färg. Tornar pat fötter àro ofragula. Den lefwer af Primula, An-

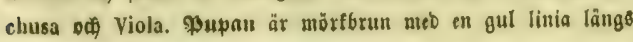




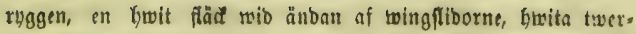
banb på abboninalregionen od) îera, något upphöloa guldo. ods filfowerpunfter.

\section{Subdiv. Fötternes lirr undertill makna. Vingner-} verne 10. Första vingnervens på bakingarne gren krökt, uti̊tböjd.

11. Bastard-Perlemorfjärilen (Argynnis niobe Lin.)

Alis anticis margine exteriore fore recto; omnibus supra fulvis nigro maculatis; posticis infra ferrugineo variegatis maculis subquadrangularibus flavis (aut argenteis), ocellis ferrugineis argenteo-pupillatis lineisque posticis duabus approximatis, paralellis fuscis. L. al. exp. 50-55 millim.

Mas nervis alarum anticarum 2 et 3 supra vix incrassatis.

Femina alis ad basin nigro-fuscis, nervis alarum anticarum aqualibus maculisque apicis pallide flavis.

Frammingarne $i$ bttre fanten näftan rüta; alla wingarne ofwan röbgula, fwartịăafiga; De Gafre unbertill rofffärgabt fanmmige med gula (eller filfweriärgabe), ingfidiga flädor od) rofffärgabe, ftlfwerpu* pillerabe oceller, famt $i$ utfanten tisenne närffåenbe mörtbruna, paral. lela linier. \&. e. Ivingfp. $23 / 16-21 / 4$ t.

5amuen bar 2 od) 3 nerberme på frambingarnes ofre fida fỏga tioctare ân be ofrige.

5youau, Gwar8 wingar äro wib bajen nåftan fwartbruna, bar wingnerberne lifformige, mett $i$ franwingarnes fpetfar blef. gula fịååar.

Syn. Pap. niobe Lin. F. S. и. 1067. Fabr, E, S. III. 1. 147. 432. Iläbn. Pap. 1. 14. 1. 61.62. t. 194. i. 961. 962. (var.) Toxt. 12. 17. Argynuis 0chs. Schm. I. 1. 83. IV. 15. \& 118. Treitsch. X. 1. 16. Dalm. Pap. Sv. 67. 4. Zett. Ins, Lapp. 895. 4. God. ot Dup. Lepid. II. pl, 7. 1, 3-5, II-S, Text, I, 36, \&. 32. 1. 142146. ( $\mathrm{var}$ ). 
Var. b. $\left\{\begin{array}{l}\text { Alis posticis infra maculis argenteis. } \\ \text { Bafwingarne undertill flfweriladabe. }\end{array}\right.$

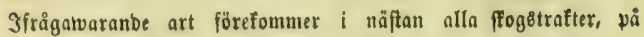
torra bacfar odh betesmarfer, Gwareft ben ifpnnerbet befüfer ber toxxanbe arter af Thymus od̆ Viola. Sällan finner man ben på ängar oç fanfa fällen. Den är almännare $i$ fäbra Sme: rige, ân $i$ meblerfta od norra, ehuru ben lifwäl äimen blifwit an.

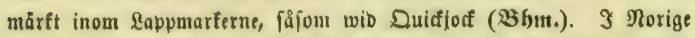

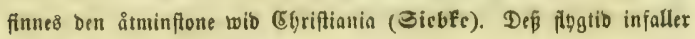

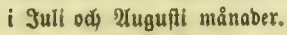

Då benna fiäril få lifnar fölíanbe art, att ben blott föga ffilifes berifrion, är bet öpwerfiöoigt att lemna en omptänoligare befrifning, utan upyiöres bär enbaft en iemförelie mellan be partiex, hwaruppå båba arterne lättaft friltas från bwaranbra:

\section{Arg. niobe.}

Stärilen i allmänbet minbre. Rrop: pen föga färre än yos Arg. latonia.

Zrammingarne $i$ utfanten rurbabe, đller nảftan rāta, fnapt märf̆s bart injwängba.

Zärgen ofman ogftrare röbgul, if́nnerbet bos bonan.

Wingarne ofwan wib bajen monrs: bruta, ל०ఠ bonan näftan fwarta.

28aftwingarne unbertill oftaft med gula, fällan meb fllfwerfärgade ศึåđar.

2.afmingarne unbertill (iignnerber hos bar. b.) utöfwer mibten farlt rofffärgabt flammiga på

\section{Arg. adippe.}

Ftäriten $i$ allmånbet padrre. Srop. pen af nåfan famma ftorlef fom hos Arg, aglaja.

Framtringarne $i$ utfanten mer. enbels temligen topligt in fmăngoa.

ร̧ärgen ofwan lị̂tigt röbgul.

Wingarne ofwan wio bajen wis. ferligen mörfare, men lifwäl ide bruna.

Bafmingarne unbertill oftaft meb filimerfärgabe, fällan neb gula flåçar.

Bafwingarne unbertill blott wio bajen od utmeb intanten roft. färgabt faanıuiga, men matt 
Glefgul botten, ' fa att flärtlen לằr är mörfare, ån Arg. allippe. \&ångs utfanten af bafmingarnes unbre fíta lōpa nảra Grwaranbra tmenne parallela mörťbruna, tyoliga fired.

รูannenళ 2 od 3 nerver på frams wingarneb öre irba lifa med, eller föga tiocare än be örriga. Framivingarnes fpetfax bos Gonan ofwan merenbelo lijuft gulplàciga.

Fläđatne på bafwingarnes unbre fiba metendels minbre.

Det furarta fireck, bwarmeb be liăfar, fon biloa mibtelbubet p\& Eafwingarnes unbre ftba, inåt begränfab, ofta ợjå det flect, byarmed famma fäđåar utåt begränjas, tholigt ođ̆ fintpare marferabt ån loos Arg. adippe.

Şannen ăx till mingarnes öfre foba Ifujare röbgul än

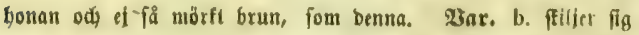
endaft berigenom att bnfwingarne på unbre fiban hafwa flitrer. färgabe $i$ ftället för gula fläđar. Den år fälüntare, ån ben normala formen.

Rarveu är brunagtig med en hyit, fmarifantab linia längs ryggen ođ̆ en fwart längs lywarbera fiban. (Gmellan igggen है od flbans linier får på bwarje fropperring en tretantig buvit fläđ. Tornarme äro blwitagtiga. Riffom Deß fauılügtingar Isfwer ben företräbeobis :ia arterne of flägtet Viola. 


\section{Allmänna Perlemorfjärilen}

(Argynnis adippe Lin.).

Alis anticis margine exteriore nonnilil exciso; omnibus supra fulvis nigro maculatis; posticis infra virescenti-flavis maculis subquadrangularibus argentois (aut favis), ocollis forrugineis aryenteo-pupillatis lineisque posticis dualus distantibus forrugineis, interiore obsoletiore. L. al. exp. 5:i-6!i millim.

Mas: alis anticis norvis $2-3$ in medio valde incrassatis.

Femina: alis anticis norvis inter so arqualibus maculisque apicis fulvis.

Frammingarnç ถูttre fant något utfuren; alla wingatne ofwan röbgula, fwartfäatiga; be bafre unbertill grönagtigt gula meb filfwer -

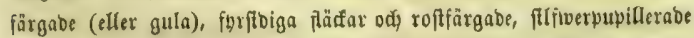
occller, fant $i$ utfanten twenne toibt från fywaranbra fitido, roporuna linier, af hivilfa ben inbre är otgbligare. \&. Ł. wingfp. $2 \frac{1}{4}-$ $2^{11 / 16}$ t.

Santuen Gar 2 ợ 3 nerberne på frammingarnes öfre fiba fartt tilltiofnabe.

Syouan bar wingmerverne finbemellan lifa ody $i$ frambin: garnes fpetfar röbgula fläนึar.

Syn. Pap. adippe Lin. S. N. I. 2. 786. 215. Valor. E. S. 11. 1. 146. 448. llülon. Pap. t. 12. f. 63.64. t. 17\%. f. 859.860 , t. 180. f. 888.889 . Text. 12. 16. Argynuis Ochs. Schm. I. 1. 88. IV. 15. \& 118. Treitsch. X. 1. 16. Dalm. Pap. Sv. 67. 3. Zett. Ins. Lapp. 895. 3. Ged. ot Dup. Lepid. I. 57. pl. 3. f. 2. pl. 3 secund. f. 2. II-S. Text. I. 37. Pap. Cydippe (laps. calam.) Líu, F. Sv. 1066.

Bar. b. $\left\{\begin{array}{l}\text { Alis posticis infra maculis flavis. } \\ \text { Bafwingarne undertill med gula päfar. }\end{array}\right.$

Denna art, fon öfwer allt $i$ jöbra od meblerfta Swerige od inom Slorige ảnba till Tronbhiem är allmän wå betegmarfer od ängar, fâmäl $\mathfrak{i}$ foggtrafter fom tå fãttbugo, Glir inom norbligare provinjerne tensligen färfynt, men Guru bögt den egentligen pitiger 
mot norben är n̈nmu ide utrōnt. Dod funes ben gå längre mot norr aีn föregående. Brof. Betterítebt anför ben fåfont fällan ïore=

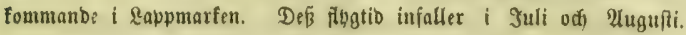

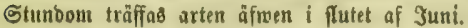

Mrtbeffr. Qhatennerne bruna, unbertill ftötande $i$ röbgult. Bal=

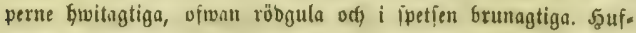
wub od rogeffölo röbgula, tätt lubna. 2(6bomen ofwats fwart, ber od) Guar med röbgult puber ody lubb; unbertill röbgulag.

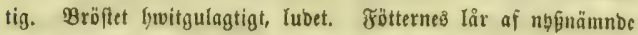
färg, men tibier ods tarfer röbbrunta. Frammingarne i uttre fanten märffart infwängba ; be bafre $i$ jamma fant fmåtanbabe.

Sannic: Mingarne ofwan röbgula meb fanma fwarta tefoning fout los famflägtingarne, uffanterne med twenne, bwar.

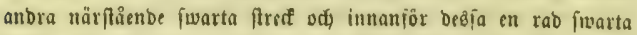
Galfmånar, jom fammantyänga meb knwaranora ody meb nämnbe froarta ftrext, fi̊ att berigenum fläfor af grunbfärgen inneflutas. Unbertill äro frantwingarne fåfom på öfre fibant, men wings fpetjarne äro lifut gulagtiga me grönagtiga, unberfunoom filfo twerfärgabe lăfăar. SBafwingarne äro unbertill blefgula med mörfare nerber od hafwa nårmare bajen blefgröna, jamman: fatande fäammor. Deras - frantob àr ftunbom filfmerglänjande od) wib bafen ftå 3-4 fmàrre filfwerfärgabe flädar, berefter uti en twertab 3:ne ftörre, af Ghvilea ben meblexfta är ftörff od inåt od) utåt begränjab af en fwart linea; berpå fôlfer ett wås gigt banb af fllfwerfärgabe, inåt od utait fwart eller mörfbrunt

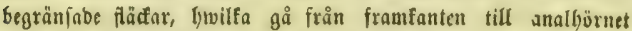
ody äro af olifa ftorlef. Längs utfanten går en rab af trub. bigt trefantiga, inåt med roftbrunt begränjabe, flffwerfärgabe

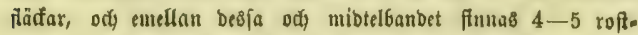
färgabe flâtåar, tifl ftörre eller ntinbre antal förifebde lywarbera

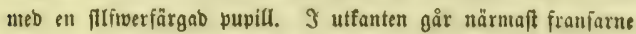
ett fiwarfagtigt foreff ody innanför betta, wib perlemorfääcornes bttre fant, ett annat blefare rofterunt; ftunbom ganffa otbbligt. Franfarne ăro blefgula meb bruna fläfar. 
Syonan àr ofwan af buftrare färg odis fyar alla nerberme på frammingarne normala. För öfrigt litnar bon haumen.

Bar. b. lifnar förut beftrifna normala form ods är fälle inntare äı benna. Bafivingarnes unbre foba hafiwa blefgula fläcfar $i$ fället för be filfwerfärgabe. Enbaft be ropfärgabe กิäçarnes pupiller äro filfwerfärgabe.

Rarven är brungrå meb Lytwit, affruten regglinia, fwart: agtiga finebftred pa floorna od) Jjujare tornar. Defi mober= plantor äro arterne of flägtet Viola. Wupat är brungra med blåagtiga đller filfwerfärgabe fläfor. Earven öfwerwintrar.

13. Stora Periemorfjärilen (Argymis aglaja Lin.)

Alis anticis margine exteriore rotundato; omnibus supra lulvis nigro maculatis; posticis infra favis, viroscenti obductis, maculis 21 ovatis argentois. L. al. exp. 55-60 millim.

Frammingarne $i$ bttre fanten runbabe; alla wingarme ofwan röbgula, imartiläctiga; be bafre unbertill blefgula, ôfwerbragne meb

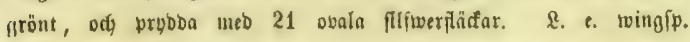
$2 \frac{1}{4}-2 \frac{1}{2}$ t.

Syn. Pap. aglaja Lin. F. Sv, 1065. Fabr. E. S. III. 1. 144. 142. Hiübu. Pap. t. 13. f, 63. 66. Text. 12. 15. Argynuis Ochs. Schm. I. 1. 91. IV. 15. \& 119. Treitsch. X. 1. 18. Dalm. Pap. Sv. 66. 2. Zett. Ins. Lapp. 893̈. 2. God. ot Bup. Lopid. 1. 54. pl. 3 secund. f. 3. II-S. Text. I. 37.

Bar. b. $\left\{\begin{array}{l}\text { Alis supra fusco induclis. } \\ \text { Wingarne ofwan öfweroragna med fwartbrunt. }\end{array}\right.$

Syn. Papilio amilia Acerbi Voỵ. au Cap. Nord. Vol. III. 175. pi. 15. 1. 1. 2.

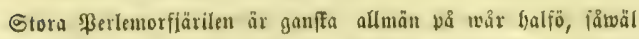
i föbra jout norra provinferne, lifuăl alluännaft i be fürta. Brof. 3ettexiscot har hemföt honom från Tornea Rappmarf, ber äfwen

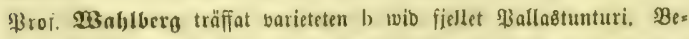
sesmarfer od fog foängar, tillodymeb be fanfare af beşa, är arten

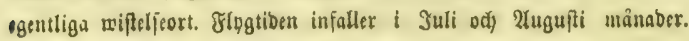




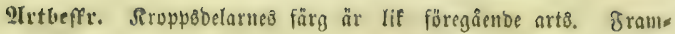
wingarnes bुttre fant är runbad od) Gafwingarnes fmåtanbab.

Somute: 2 BingarmeB färg ofwan o() frammingarnes unoertill är lif \&llmänna झ̧erfemorijärilene, enbaft med bet unbantag, att $i$ fromwingarmes fpetfar unbertill, länge utfanten, finnes alltib ent rad matto fllfwerfärıgobe fläfur. Bafwingarne äro undertill bleft Bula, men från bafen, wiot utôfoer midten, öpuerbragna med blef=

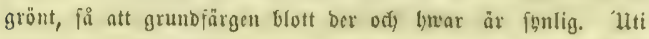
Detta gröna fält finns i famma orbning fom hos föregånenbe

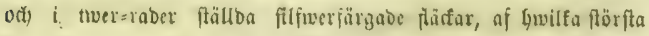
belen äro äggformiga, wiot från flwarautora ffilon och fafua märfbarare mörfbrun eller jmartagrig begränğning. ¿änģ

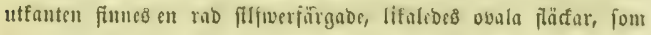
begränjas utait af en grönagtig linia ody inât af fläatar med famma färg. S ijelfiwa utfanten går elt fivartagtigt ftrecf. Franjarne äro liuft gula med mỏrfare flăfor.

Sonau lifnar till färgen bannen.

Earves är fwatt med juarta tornat, gul r乡gglinia ods röba flooflärfar. Såjom dể famflägtingar teịer ben pai Viola

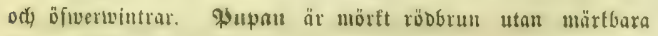
taggar. (Sdjif.).

\section{Silfuerstreckade Herlemorfjöriles:}

(Argynnis paphia Lin.)

Alis supra fulvis nigro-maculatis; posticis infra viroscentibus fasciis transvorsis subinturruptis argenteis. L. al, exp. $60-65$ millim.

DEingarne vitwan röbgula, fwartflärfiga, be Gafre undertill grön. agtiga med twerguiende, ber orl) fiwar ajbruma, filfwerfärgabe twer. bant. \&. e. wingip. $2 \frac{1}{2}-2^{11} / 16$ t.

Syn. Pap. paphia Lin. F. Sv. 1064. Vabr. E. S. III. 1. 142. 438, llïbn. Pap. t. 14. f. 69, T0, t, 190. f. 935. 936. var. Text. 13. 19. Argymuis Oehs. Schm. I. 
1. 96. IV. 15. \& i87. Treitsch. X. 1 1s. Dalm. Pap. Sv. 66. 1. Zett. Ins. Lapp. 89.ّ. 1. Goul. ot Dup. Lepid. 1. 51. pl. 3. f. 1. pl. 3. secund. f. 1. II-S. Text. I. 30.

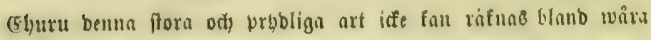
allmännafte flärilar, förefoumer ben lifwäl ef fälajgnt ber ody gywar

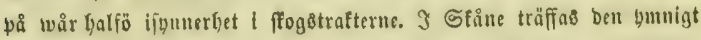

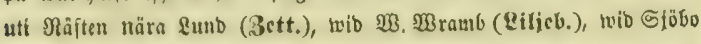

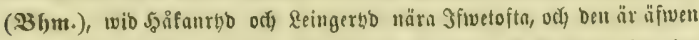

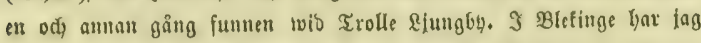

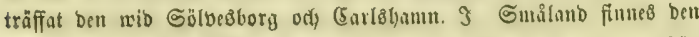

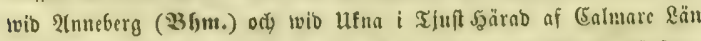

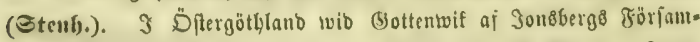
ling (હtenb.) förefonmer den. Rifafí twid Stoflyolu. Snom Norige bar, ben blifwit anmärft wib Efrtiftiania, Drumumen od EDsberg (Sicbfe). Sglvareft ben mot norben upplÿr är obefant, men $i$ norra belen of $\mathfrak{B}$ eptmanland trâffaß ben ei (Frebrida)i.). ßrof. Betterifebt upptager ben Gland Sappffa infefter fäfom fäf.

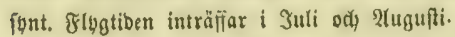

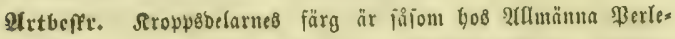
morflärilen. Frammingarne ăro $i$ utfanten utffume; Gafwin= garneß uttant fmåtanbab.

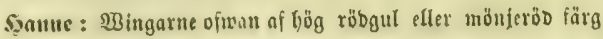
med be imanliga jixarta teçningarne. De fyra förfta nerverne på frammingarne äro fturft tilltiocfunde odi) fivarifiällige; ben femte merenbels något minbre. Utnbertifl åro framıingarne blefarc, för $\mathrm{ofs}$ rigt näftan lifa färgabe od) teffnabe font på öfre fiban, ment Y)afrea grönagtige fïăfar i wingipet farme. Bafningarne äro unbertill gröna med fura flipwerfärabe (iafe af fläcfar teftaiende) twer: band, af Glvilfa be 2:ne, fon äro närmaft bafen, ife finna wingarnes inbre fant ody bet 3:bie går från framfanten till analo bôrnet, ber bet förenar fị me bet fierbe, four löper längs uts

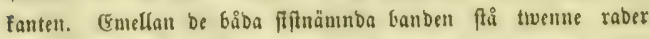
förre, runbabe, mörft grönagtiga fläđar, quvilfa untingen alla, 
eller blott nagra, tiaböranbe fifta raben, omflutas buwarbera af en filiwerfärgab sing.

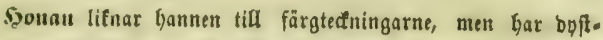
rare grumbiärg ofiran od) alla nerverne på framwingarne nor: mala. Defutom ät Gafwingarnes unbre floa lintigare grön, fuubon meb purpurglans. De fwarta tefningarne på wingar. пев öfte fioa äro beriemte gröfre.

Earven, fom lefwer på Urlica, Rubus ody Viola, air brun uteb gula tornar. De längre halstornarne äro bruna och längs roggent går en liusgul linia, fom $i$ mibten belas af en annan jwart ợ på Gwarbera fiban begränjẩ af en mörfbrun. Pupat är frunagtig meb röba fli̛far på mellanfroppseregionen oḍ fwart. agtiga $\mu$ å toingftioorne.

\section{Sliggtet: Vanessa *). Faвr. et Aбcr.}

(Aglais DALM.)

Antennæ longiores, rigidæ. Clava cylindrica oblonga, distincta ot determinata.

Palpi capite multo longiores, adscendentes, apicibus subconniventibus, ad latera squamati, infra denso birsuli, triarticulati. Articulus modius longissimus, leviter compressus; ultimu $_{s}$ angustior (basali xqualis), conicus apico obtuso.

Oculi hirti.

Pelles antici broves, pilosissimi tarsibus maris inarliculatis fominæ artículis quinque, minutissimis, arcli conjunctis; ceteri ut in genere Melitea armati ot instructi, sed articuli tarsorum aliam habent proportionem: 1:mus longissimus roliquis fere equalis, 2:dus "eptio ot hic quarto, minimo, longior; 3:tus tertio fore requalis of unguibus quatuor, plerumquo validis, armatus.

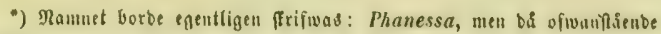
friffátt ảr allmảnt nut" get, bibegälles bet ảfiven gár. 
Alx anteriores saltem angulato-tentatx. Cellulæ discoidales costa spuria clausx.

Costo alarum posticarum $9 \mathrm{nt}$ anticarum 10, omnino ut in specichus subdivisionis secunde generis precodentis collocatix; costa nempe octava al. anticarum sompor a septima separata, e latere collula ogrediens. Costa transversa, cellulas alarum claudens, inter costam 4 ot 5 posticarum ot intor costas easdem anticarum plus minusve ovanescens, plerumque tumiditato tantum continuata *).

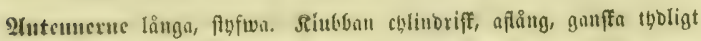
affrtilb fro̊n fisftet.

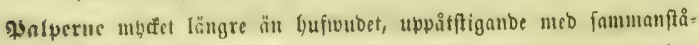
enbe fpetiar, Đå fldourna fiälliga, undertill tätt båriga, trelebabe:

-) In tahula sequente forma costo transversm alarum et ramuli ensto primœ posticarum in speciebus nostris indicatur:

A. Costa trassversa alarum anticarum cum continuatione sua subtilissime.

4. Costa fransverea alarum postiearum cun continuatione sua subtilissima.

1) Ramulus cosle prima alarum posticarum reclus apice bimueronato: $V$, alalania.

2) Ramulus coste prime alarum posticarum apice simplice.

a) Ramuius costo prima alarum posticarum reflesus: V. Cardui.

b) Ramulus cosfa primæ alarum posticarum ioflesus: V. antiopa.

c) Ramulus costæ prime alarum posticarum rectus: V. $c$ album.

2. Costa trinsversa alarum posticarum cum continuatione sua sat distincta, Ramulus coste prime alarum posticarum rectus apice simplice: $\boldsymbol{V}$. urlica et potychloros.

13. Costa iransiefsa alarum anticarum cum continuatione sus rat distincla, subcrassa. Ramulus coston primo alarum posticarum simplex rectus: V. io. 
meblerfta leben ganffa lång, lät fammantrbăt ; ben fitita leben imalare (lifa ftor mob basleben), fonift net trubbig fpets.

\section{Ş nonen lubna.}

Fïterue: be främre forta, ganffa lubna med hos hannen olebabe od) bos bouan otubligt femlebabe tarier; be öfrige fajion hos flägtet Melitea Gewäpnabe, men leoerne $i$ taricrne bafwa en an. nan proportion: 1:fta ganffa lång, näftan lifa läng meb be ôfs rige; 2:Dra lẳngre än ben 3:Dje, demna längre än 4:be, fon 'är minft; 5 :te näfan lifa med den 3:Dle od betwäpnad med 4, mers endels ftarfa flor.

2Bingatue: åtminfonte de jräntre, winfligt tanbabe. Difffălten futn? medelft en falfe twernerb.

शerveruc på bafwingarne 9, od) på framuningarne 10, allbeles fia ftällbe fom hos arterne $i$ anbra feftionen af föregåenbe flägte, nemt. få; att 8:be nerven på frambingarne alltio utgår från ban af bifffăltet, ffil fro̊n ben i:be. Twernerven, jom tillfus ter bifffälten, emellan 4 odh 5 nerverne pâ bafwisigarne odts emellan famma nerver pá jrambingarne mer eller ninbere förs fwimanbe, merendels blott fortfatt medelft on uppionällning.

faithöranbe fiärilar förefomma $i$ tweme scuerationer om arct

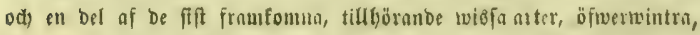
få att be unber flara od milbare bagar om wintren utfomma

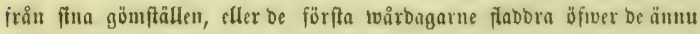
Ivifna, från frön fuapt befriabe fälten. Deros larver fafna turnar på förfta froppsiringeu, od hafiwa på 2:bra od) 3:ofc bögit fura, men på be öfriga altio 6 fäbana, bwilla äro fthima, jwetifiga ods mucfet greniga. (En bel äro feabliga för triẗben, ment meb unbantag af besfa arter, fuma be öfrige anfes offabliga, då man här iffe anmänber beras moderwerter, fafom i utlumbet. \$upan har ett par förre fpetfar $i$ hujwubeto fräure del oci) ett par minove pâ ruggen af huvarie

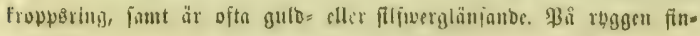

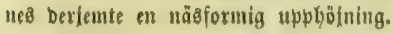




\section{A. Pramvingarnes tremerv tillika med dess fortsitt- ning ganska fin.}

1. Bakvingarnes tvernerv tillika med dess fortsättning ganska fin.

1) Grenen på bakvingarnes första nerv i spetsen tvảtaggig.

1. Admiralen (Vanessa atalanta Lin.).

Alis dentatis, supra nigris, anticis maculis difformibus apicalibus albis fasciaque obliqua in medio fulvo-rubra; posticis ad marginom exteriorom fascia nigro-maculata ojusdero coloris. L. al, $\exp , 7 \mathrm{Z}-75$ millim.

Wingarne fmåtanbabe, ofwan fmarta; be främre meo olifformiga Gwita Aăfar $i$ fpetien od ett gulröbt banb jnebt öfwer mibten; be bafre wio utfanten mes ett jwarriläđtigt band af famma färg. \&. e. twing $\left[\right.$ p. $2 \frac{7}{8}-3 \frac{1}{16}$ t.

Syn. Pap. alalanta Lin. F. Sv. 1060. Nabr. E. S. III. 1. 118. 362. Ilïbn. t. 13., f. 75. 76. Toxt. 14. 2. Vanessa Oclas. Schim. I. 1. 10\%. IV. 16. \& 127. Zett. Ins. Lapp. 894. 4. God. ot Dup. Lepid. I. 99. pl. 6. f. 1. II-S. Toxt. 1. 41. Aglails Dalm. Pap. Sv. 65. 6. 2lomiralen är en af wira tradrafte flärilar ody förefommer om

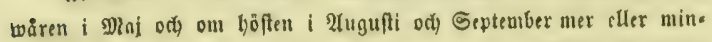
bre aflmän 1 föbra od meblerfta Gwerige, ifpunerbet i träbgårbar

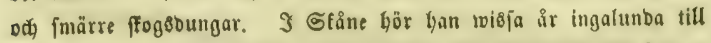
fâluntheterne, anbra àr fan man beremot ofta förgäfweo föfa bonom på ftällen, ber ban elfeft brufar wara allntản. Э Småland är han

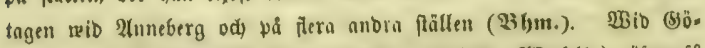
theforg od) på WBifngso år han äfiven anmärft (2Bahlb.), äfwenfå wib Slođtholm. Snom Norige är Gan trâffab wib (E)riftiania (Sicbfe). รูuru liogt han figer mot norben of wår halfö ầ ännu ef utrönt. Brof. Bitterfteot anjör yontom jâfom fällynt af Eappffa infefter. Lti norra belen af Befimanland förefomaser ban lifwäl ide (Fre: Drichion).

Irtbeffr. 2lntennerme fwarta neb huvitagtign ringar. Slubban i fpetîn gulngtig. Balperne ofwan jwarta, pâ floorne brwitag. tiga, unbertill brungulagtiga, tätgåtiga. Suftwub vd raggfỏl. 
bell fwartlubna. X6bonen ofwan [wart, unbertia bwitgulagtig. Bröftet gråblarfigt, lubet. Bafwingarne från baโen, längร in. fantell fwartluona. WBingarne tanbabt wågige, be fräurte med in förre tanb $\{$ utfauten.

Sonute of houa: Wingarne ofwan fwarta, lywarbera med ett gulröbt bautb, flwilfet på frommingarne går från framfan. ten fnebt öfwer mibten till bafre börnt, lifwäl utan att binna fielfwa fanten, nen beremot pâ bafwingarne föliłer utfanten ợ är bär i mibten förfeot meอ en rab trefantiga frarta flätar, famt $i$ allalgörnet prybt med ell blåaglig. I framtringarnes fpetjar finnas 6 ftörre orly minbre buvita fläafar af olifa form, bels nilånga, bels rumba. Länge utfanten af famma ivingar

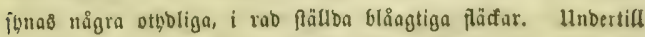
äro framusingarne tefnabe fåfom på öfre fiball, men twingipetfarna äro brunagtige odh omfring be Gyvita fläfarne funnas fwarta ringar, äfwenjou nărmare bafen några blåagtiga frect. Baftvingarne äro undertill brungrå med ljufare od) mörfare flammor famt bafma $i$ mibten of framfanten on gulagtig fläđ, $i$ biffen en fwart tefning, lifnanbe 686 , od mot utfanten en eller flera, osboliga ocellformiga flärtar.

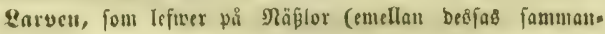
fpunna blab), är fort od, tloff, töttärgab, gulgrön, fwart ellet brumröo med gula tornar od ett gulf, af halfmånar beftåenbe

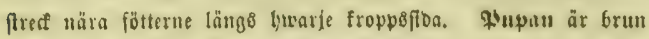
cller gra meb undet trubbiga fpetiar $i$ buimubet od fiera, wans ligen gulbglänjanbe taggar på nfobominalregionen.

2) Grenen pá bakvingarnes fürsta nerv i spetsen enkel. 2. Damen (Vanessa cardui Lin.).

Alis dontatis, supra lulvis, rosoo tinctis maculis inæqualibus atris ot albis; posticis infra marmoratis ocellis quatuor, I. quinque. L. al. exp. 55-70 millim.

Binģarne fmåtanbabe, ofman rōbgula, ftötanbe i rofenrốbt, meb förse ody minbre firarta odj huitu flüfar; be bafre unbertill marmorerabe ody proboe med $4-5$ oceller. f. c. wingfy. $2 \frac{1}{4}-213 / 10$ t. 
Syn. Pap. cardui Lin. F. Sv. 1054, Pabr. E. S. III.

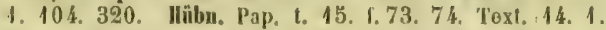
Vanessa 0chs. Schm. I. 1. 102. IV. 16. Treitseh. X. 1. 19. Zett. Ins. Lapp. 894. "3. Cien. et Dup. Lepid. I. 102. pl. 5. secund. f. 2. H-S. I. 30, f. 157. 158, var. Text. I. 41. Aglais Dalm. P’ap. Sv. 65.7.

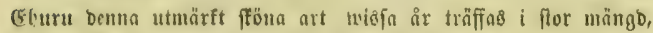
fan ben ide anfę för allun̈n. De ìefta år träffas blost få excms

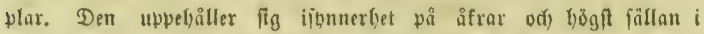
ffogstrafterne. Detta är orfaten bwarföre ben är allmänntare i Gfä.

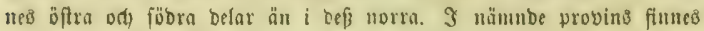

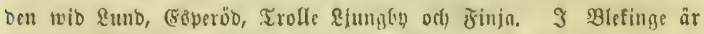
Den atmärft wid (Sarlafrona od) Sölucaborg. $\quad 3$ Smailand är Den

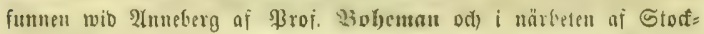
bolm bar fag fielf tagit ben. WBio Yhalnuwif nära Drotminģlyolm

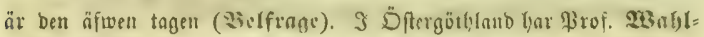

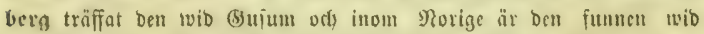

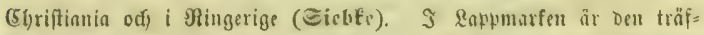

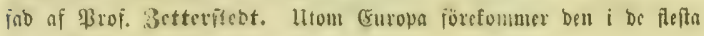
länoer af आfen, 2(irifa oç) 2(merifa.

Wirtbefrs. 2(ntennerue mörtbruna, gleft linitpubrabe. silubban i fpet=

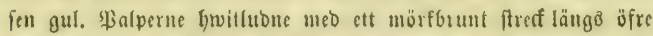

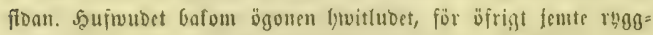
ftölbci frungult, lubet. आcoomen ofman fwast, i lebfanterne od unbertill röbgur. Bröftet grâlbnitt, Inoet. Fötterne bwitgula. Bafwingarne ofunan från bafen länge inde fanten benurna meb längre, röbubla båx. WBitgarne i utfanterue fafom hos 2(omirnlen.

Saute ori) boua: Wingarne ofman röbgula meb rojenröb anftrbening; be främre i minglpetien, fuebt ofmet från framfan=

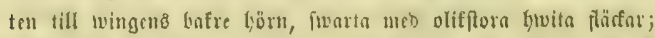
länga utfanten löber etl otyoligt, lywitugtigt ods aforutet ftreff

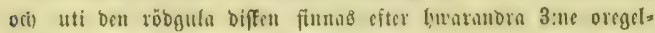

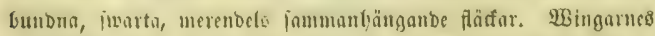

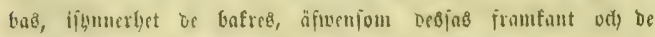

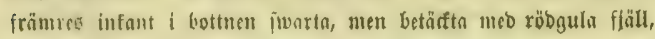


Guraraf fărgen Gär är mőrfare. Till fölle buäraf Gillbar bet röb. gula på baftwingarne enbaft ett twerbanb wib utfanten, Givilfet ide bimer feelina framfanten. Lti betra finnas $4-5$ förre runda, fwarta fläđar ocl) längs utfanten twenne parallela raber furarta, olifformiga Tläfar, of şwilfa de inbre äro vå tweren obali ody nerendela fammanţängande, be bttre beremot förenabe bfort genou en fill, fwart linis. Twenne af de förftnånuba, luvilfa äro närutaft anallörnet, itöta i Glåts. Unberrill äro frambingane tefunde fijoun frả öfre floan, utom reingipetfon

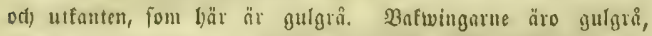
marmorerabc med frunt of (s) witgult, famt bafma längs ufaus ten uti ett otholigt, furitt banb en rab af ftumbour famman.

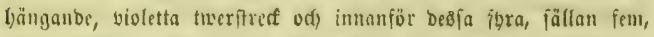

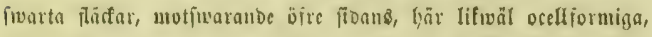
iå att till ett fourre cller minte nital af bem, är blwarbera om: gifuen af en röbgul od en fwart ring font $i$ mibten uâgot blä pubrab. Franjarne äro lowita meb fiwarta fläfar.

Earven är mörfare cller ljujare fwartgrå meb gula farcf, flisfar cluer punfter od) forta tornar. Den lejuer pai flera flange

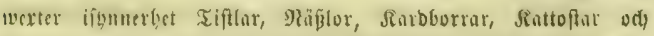

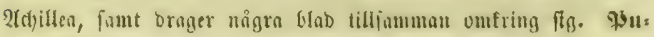
paแ är affegra, elier brun med twå trubbign uppb̧öjninģar $i$ fuimubet, od) flera törgstba taggar ed) fuaptormiga fpetjar tå abbominalregionen.

\section{Corgenanteln (Vanessia antiopa lin.).}

Alis angulato-dentali, supra castane-niģris limbo lato, flavoalbido, sirio adjacente macularum foerulearum. L. al. oxp. $70-78$ millim.

23ingunue winfligt tanbabe, ofuan möıft fnftanisbruma med Greb,

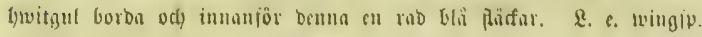
$2^{19} / 16-3 \% 10$ t.

Syn. Pap. antiopa Lin. F. Sv, 1056. Nabr, Li, S. III. 1. 115. 355, Ilüb». Рар. Ł. 16. 1. 79. 80. Vanessa 
Dchs. Schm. I. 1. 110. IV. 17. Zett. Ins. Lapp. 893. 1. H-S. Text. I. 39. Aglais Balm. Pap. Sv. 64. 2. Vanessa morie God. ot Dup. Lepid. I. 93, pl. 5. f. 1.

Denua art förefommer uti fogarne, ifsmuerlyet ber Solört ody

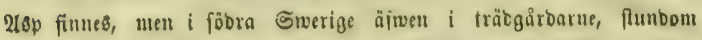

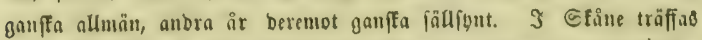

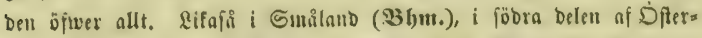
göthland (Stcub.), på Sottlanb (3ett.), wib Stoffjolm ody äfwen

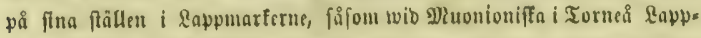
marf (3ctt.) ß̊̊ pera mellunligganbe orter ät ben lifunâl fänbigt

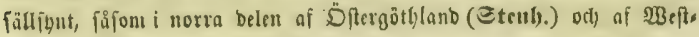
manlano (Trebrid)i.). 3 Sorige är bell anmärft wio (Shriftania,

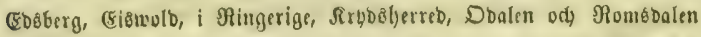
(Eirble). Flugtioen infaller menne gainger om året, i Whal od

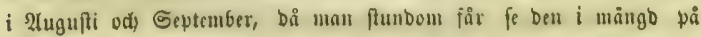

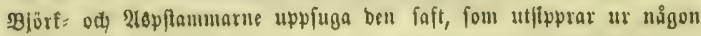
fpringa.

2artbefir. 2(ntennerne od flubban fwarta, ben febuate meb oul fpecs. \$alperne gula meb fwarta ränber o(j) unbertill benurna meb längre, fuarta f̧år. Sรufwub od) ryggfföld brunlubna. 2tboomen fwart. Bröftet, äixenjom Gafwingarne längs inbre fanten unbertill, lubue of fwarta, i fpetien gula bior ; be febuare ofwan, frin bafen utôfwer midten, betâfta meb lång, faftaniebrun lubb. Sglwarbera twingen bar $i$ utfanten en gröfre tand. Fötterne meb gula tibies ody tarfer, famt lwarti, mot leben gula lär.

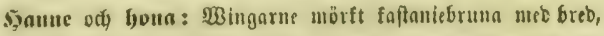
Jjuรึดul eller hwitgul forba, fom är mer eller minbre beftröbo med fwarta atomer. Straxt imnanfor benua är grumbfärgen

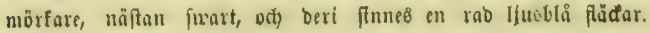
శraumsingarne bafwa $i$ framfanten fmå, gula twerftreç od) när= mare jpesfon twenne förre, gula fläfir. Hubertill äro rain. garme fwartbruna med folfwart wattning od) est tybligt, wågigt twerftred of famma fârg jfwer baftvingarnes biff. De trentue gula täåarne $i$ frambuingurne främre fant, äfwenfom bingare 
nes borba, äro blefare ofn pa Dfre flban od ben febnare har ftörre, fwarta atomer, Guilfa ifpnnerbet lifjom på oire fiban äro tätiłåenbe på wingfanterne grôfre tänber. $\Im$ biffen af baf= wingarne finneø̊ en minbre, gul fläcu.

Earycu, jom är fwart med fwarta cornar od förre röba flärfar, lefwer colonietwi yå \$Biürf, 2(8p, ßoppel od) plere \$ile arter. \$upau är affegrii eller brunagtig med fwart fdyattering

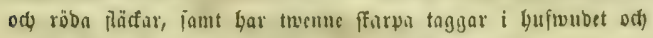

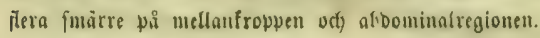

\section{Vinbirs-Fuelnsen (Vanessa c album Lin.).}

Alis acute angulato-dontatis, supra fulvis nigro-maculatis; infra cinereo-luscis plus minus albido marmoratis, posticis in medio $c$ albo notatis. L. al. oxp. 50-55 millim.

WBingarme biupt ufferma orf) groitanoabe, ofixan röbgula, fwart. fläafige; unbertill gråbruma, mer eller minore marmererabe med huvitt, be Gafre $i$ mibten teffunde meb ett fyritt $c$. \&. e. mingip. $2-2 \frac{1}{4} t$.

Syn. Pap. c album Lin. F, Sv, 1059. Nabr. E. S. III. 1. 124. 380. IJïbn. Pap, t. 19. Г. 92, 93. Text. 16. 10. Vanessa Dehs. Schin. 1. 1. 125. IV. 17. \& 129. Mett. Ins. Lapp. 864. 3. God. ol Dup. Lepid. I. 85. pl. 5. tert. f. A. pl. 5. f. 3. II-S. Toxı. T. 39. Aglais Dalm. I'ap. Sv. 65. 5. Hoila c-fjarilen. Dahlb. Sk. Ins. Skada ocls Nylla. p. 153. 107.

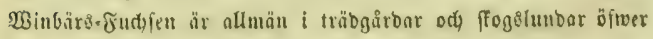
allt $i$ Swerige odf Rorige mo unbantag ni be norblignfte provin:

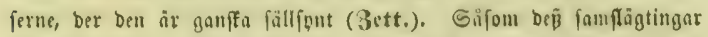
förfommer Den $i$ tneeme generationer om åres, $i$ פla

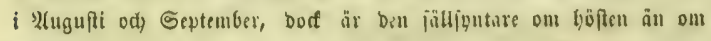
sviren.

2frtbefr. 2tntennerne bruแa, unbertill of!bligt fuwitringlabe. SRlubs

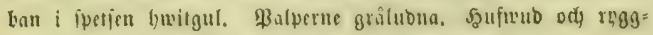
fölo röbbrunt lubur. 2l6bouten ofiwan imartagtig, unbertill pubrad mé röbbrunt. Bröftet brungrålt, lubet. Fötterne grå

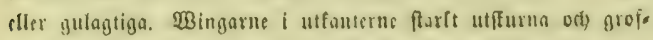
tandabe; de bafre ofnan med lânga rodgula bir lä̈gd alioment. 
Syanne ody bona: WBingarne ofwan röbgula meb en freb mörfbrun lift $i$ uffanterne, imnanför Gwilfen på bafwingarne går en amnan bylif, ffild från ben bttre genom en rab liufare röbgula pă̊far. Frambingarne bafiwa $i$ framfanten twenne tweritåenbe, fiurre, montfloruna, od $i$ biffen 5 minbre, runbabe od fwarta

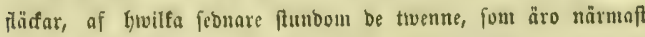
bajen ody framtantell, meb lywarambra fammantränga. I baf.

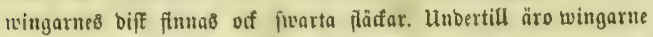
fro̊n bajen till mibten mörfare gräbruna, mer eller minbre watt= rabe meb Givitt, ftunbon enfärgabe; berefter bititgula meb ftörre o(1) minore bruna atomer od) fmiffterf, men flunbom äfwen ens fürgabt mürfgri̊. Şwarbera Gafwingen har $i$ biffen en fywit fi: gur, lifnanbe eft latinfft c, fom ruänber ben fonvexa belen àt wingens infant.

Den utmåxft wađ̛̣a latven ät till främre bäliten mörfgul od) till ben bafre quit eller hwitgul, med mörfßula ody hivitgula tornar, fwarta fläfar ods ftred; på fiborme brum meb $1-2$ Migiga, gulagtiga ftred ofwer fötterne. Den lefwer på $\mathfrak{B}$ in=

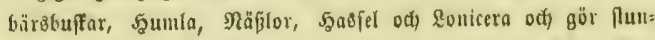

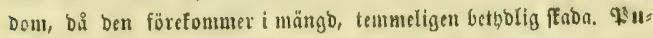

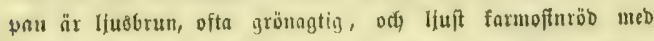
furnta orf gula freft od) fläfar, famt bjupt infuren mellan hufwubet och abboutinalregionen.

\section{Bakvingarnes tvernorv tillika med dess fortsättning} temligen tydlig.

\section{Rissel-Fuchsen (Vanessa urticie lin.)}

.lis angulato-dentatis, supra fulvis nigro-maculatis, anticis macula unica majoro of duobus minoribus rotundatis, nigris in disco; posticis supra ad basin niggris, infra nigris fiascia flavida, nigro ruscoque undulata. L. al. oxp. 50-55 millim.

Bingarne groftandabe, ofwna röbgula, jwartpäđfiga, be främre

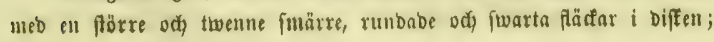


be bafre ofman wib lajen fwarta, unbertill froarta meb eft gulagsigt, fwart od [rumbattrabt twerbanb. \&. c. Ivingip. $2-21 / 4$ t.

Syn. Pap. urtica Lill. F. Sv. 1058. Nabr. E. S. III. 1. 122. 374. llî̉ı. Pap. t. 18, f. 87-89. Text, 15.7. Vanessa Ochs. Schm. I. 1. 120. IV. 17. Zett. Ins. Japp. 863. 2. Hool. et Dup. Lopid. I. 91. pl. 5. secund. fo 1. II-S. Toxt. I. 40. Iglais Dalm. Pap. Sv. 65, 4. Nässelfjüriln Dahllb. Sk. Ins. Skadia och Nolta p. 152. 106. Bar. b. $\left\{\begin{array}{l}\text { Alis supra obscurioribus, infuscatis. } \\ \text { Baingarne ofwan mörfare, brunagtiga. }\end{array}\right.$

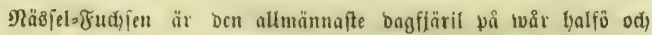

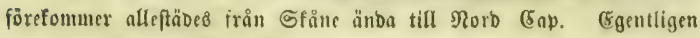
lyat bell twentue generntioner om arret, men bes̊ fa bafura iffe, fåom De fiefta anbra urterne, beftâmon tiber, utan utwerflas beín jommaren igenom, få att flärilen träffas frỏn 2(pril till flutet of September eller October måmaber. Q̈liven fair man fe bonon ivarfa bagar

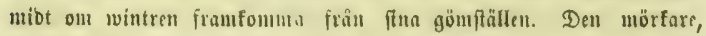
i Grunt fötanbe barieteten, forn för ofrigt lifmar ben normala for: men, är från \&apymarterme, Der Den mera ofta träffias (3ett.).

2rtbeftr. Intennerne mörforma, fint futritringlabe. Rlubtan i fpetjen gulagtig. \$alperne röbgula, $i$ fpetifen fwarta ody unbers till gråagtigt eller fwartagtigt håriga. Şuflvub od) rẹguffuld lius̊brunl)iriga. 2(6bomen ofwan fwart, unbertill röbgulagtig. Bröftet tütt fwartlubet. Fôtterne griagtiga. MBingarne $i$ ut. fanterne juåtanbabe, Gurarbera meb en gröfre oril ftörre tanb. Bafwingarne froin baicn utöfiner mibten fenuxina med guls Exuma hår.

Somue orh boua: Wingarue ofwan röbgula meb jwart, af blå flärfar prţob forba närn utfanten, $i$ byulfen ater löwer

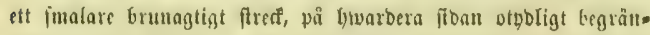
labt af grunbfärget. Framuingarme lyafwa viwan $i$ främre fanten trenne ftürre, fwarta fläfar, mellan limilfa grunbfärgen

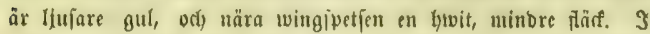
biffen af nämnde wingar fimno trenne fwarta, runbabe flüsar, af bwilfa ben fiörfta är belägen närmaft infanten. Baftwin= 
garne åro wib bafen utöfwer mibten fwarta, fâ att ben röbgula färgen blott bilbar ett twerband nära utfanten.] Unbertil åro framuingarme ofragula med brunfayatterabe foetfar, fant hafwa i framfanten od wio roten ftüre fwarta fläfar, begränfade of colfivarta lintex. Bafwingarne äro unbertil froin bafen, utöfs

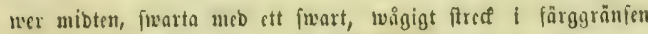

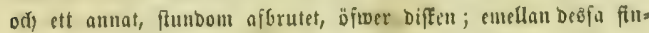

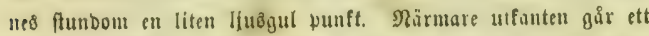
liusgult, meb fiwarta och biuna fmåīresf fint mattribt banto. Qängอ เvingarnes mörforuna ufanter löper unbertill ett fwartblått, tanbabt, finalt band.

Sarveu ar ftunbom fivartagtig, funbour grönagtigt grå mes gula ord gröngula, mer eller minbre tholiga fired och forta tornux, Den âr almän bela fommaren igenom på Nä́lor, Guilfa ben $\mathrm{i}$ grund förîtör, ifgnnerbet fom ben lefwer coloniewis. भupau är lijuft eller mörft biunagtig meb gulopunfter ody forta taggar.

6. Körsbirs- Fuchsen (Vanessa polychloros hin.). Alis angulato-dontatis, supra futvis, nigro-maculatis, anticis maculis quatuor subæqualibus, niuris in disco; omnibus infra nigro-fuscis lascia communt Alavida, nigro luscoque undulata. I. al. oxp. $60-65$ millim.

WBingaı greitandabe, ofman röbgula, imartiärfiga, be främre

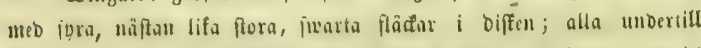
fwartbruma med ctt gomenjamt gulagtigt, af fwart od brunt wattrabt G.and. \&. ค. wingip. $2 \frac{1}{2}-2^{11} / 10$ t.

Syn. Pap. polychloros Lin. F. Sv. 1057. Pahr. E. S. III. 1. 121. 372. Ilïbn. Pap. 1. 17. f. 81.82. 'T'ex1. 13. 3. Vanessa Uchs. schim. I. 1. 114. IV. 17. Treitseh. X. 1. 20. Fod. of Dup. Lepid. I. 88, pl. 6. I. 2. Doist. teon. 125 pl. 24. T. 1. II-S. Toxt. 1. 4.0. Aylais Dalm. P'ap. Sv. 65. 3. Folisen. Dahlib. Sh. Ins. Shadia och Nylla p. 15̌1. 105.

פgar. b. Alis : antoriorom maculaquo unica ad marginom interiorom 
nigris; posticis margine interioro fulro, anteriori ot exteriori nigro.

MBingarne ofwan röbgula, be frümre méb 2 jwarta läafar wid frumfanten od) en onlif wib infanten: be bafte : inbore fanten röbgula, i ben främre od gttre fiwarta.

Syn. Pap. tesdudo Esp. Schm. 1. t. 73. c. 23. f. 1. p. 118, Pap. pyrrhomelona llïhn. Pap. \&. 174. ז. 845.846.

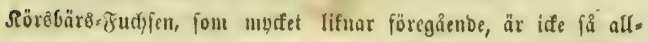
män fou benue, utan twertom en af be fällipntare inom flightet. Blott wiฐia år bånder bet, ntt man träffar bontom $i$ ftörre mängo.

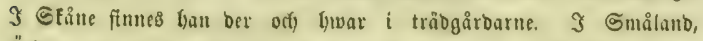
D̈fter= od) Weftergütblanb är bail äfiwen funnen (Dablb.), แten torbe fnapt gå högre än till Stodfyolut, hwareft bell off forefom.

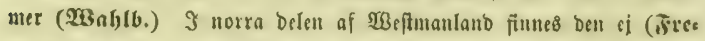
orichi.) od) är ife eller mig weterligen anmärft inom Norige. Flug. tiben infaller twenne gånger om året, $i$ פlai od 24ugufti månaber, tillochmeb nagot febnate. Barieteten har blipivet funnen wio (b) ujum

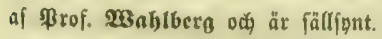

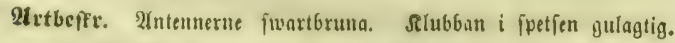
ßalperne gulagtiga, unbertifl med långa, juarta bir. Syujwubet

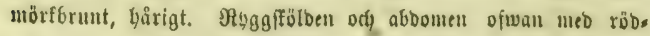
gula biri, på ben febuare glejare, grvilfen befutom undertill

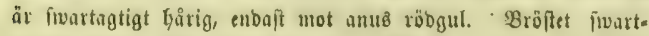
agtigt, lubet. Fötterne fwartgrå. 23ingarme me eถ grôfre tand $i$ utfanten ody Goftwingarme äro ofwan frian bajen, utöfwer mibten, bewuxna med långa, röbgula bår.

Sonute ớ f̧oun: WBingarne ofwan mörfare töbgula, än hos forregåenbe, men för öfrigt tiul färgtecfningen furtifa bennes. De främre med troenne foüre, fwarta, aptanga flüfar $i$ frome fanten oḑ en, något minbre, i biffiältet, mélan quvilfa grunto. färgen är ljuşgul. Latom be nu nämmba fiunas fọra anora, runba, fmarta fiaffir i biften, af Guilfa ben ena flio nära bufre Görnet af wingen od be öfrige från infanten $i$ fueb rigtning 
mot ivingipetfen. Lufanten är gulfrun ơh längs benfamma lo: per elt fwartorunt Ganb. Baftuingnume hajwa $i$ mibten af fram:

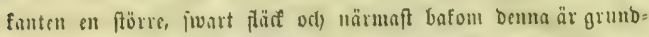

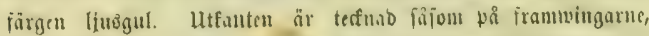
men $i$ bet fwartbruna banbet funnos llå, halfminformiga flădar. Undertill ăto alla wiugarne från bajen, utôfuer mioten, fwarts bruma med ett folpuart, wagigt ftref i färagränjen; berefter líusgula med bruna atomer, funiftreft od flummor. I framfan= ten af frambingarne fortes junma jwatta tefning fom ofwan

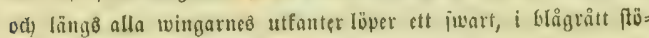

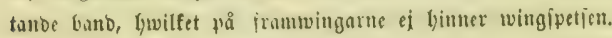

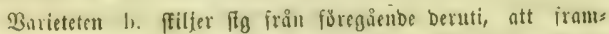
wingarne hajwa $i$ främre fanten ofwan twenne frorta fäcfar od) en bolif i inbre funten; Den feonare belno genom den förftu röbgula twermerven. De bafre äro längs inbre fanten röbgula,

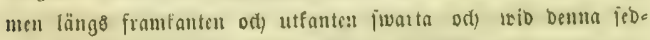
nace loper en rad röbgula fä̆dar. De blả fläđarne länge uts fantent of bafwingatnc b ofre fion, fom finnas hoo förcyående,

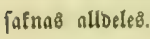

Entucu ai Glåfwart eller Grungrå med gula tornar och

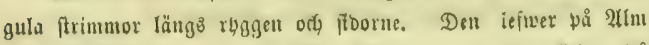

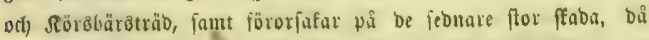
Den jürefommer $i$ mängo. Den lefwer altid $i$ ftora jälffap od

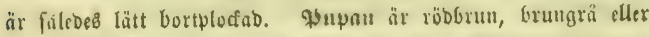
gulagtig meb gulds ods filfwerlärfar famt ffarpa taggar wả bufwub ody abobminalregionen.

\section{B. Vramvingarnes tvernerv tillika med dess fortsait- ning gansha tydlig, temligen tilltjochnal. 7. Jungfrum (Vanessa io Lin.).}

Alis angulato-dentatis, supra fulvo-brunneis, singulis ocollo magno cooruleo; anticis ad marginom antoriorom nigro maculatis. L. al. exp. $47-60$ millim. 
Bingarne groftanbabe, ofwan röbtruna, gwarbera meb en flor, blå ocell; be främre $i$ framfanten jwartflärfiga. \&. e. twingip. $1^{\top} / 8-2 \frac{1}{2}$ t.

Syn. Pap. io Lin. F. Sv, 1048. Nabr, E. S. III. 1. 88. 276, llibin. P'ap. 1. 16. ศ. 77. 78. Test. p. 14. 3. Vanessi Ochs. Schm. 1. 1. 107. IV. 17. God. et Dup. Lepid. J. 96. pl. ̈). I. 2. H-S. Text. 1. 39. Aglais MaIm. Pap. Sv. 6h, 1.

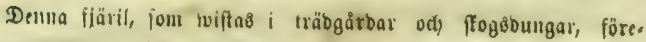
fommer, lifjom mångn andora af furc iamflägtingar, ett od antuat

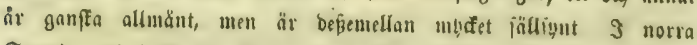

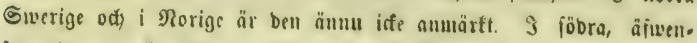
fom i meblerfta Siverige träffis don bexemot wa nifftilliga ftällen. $\Im$ Gtåne bar jag funnit ben mid Trulle \&jungुbs, Dppmanta, 5örtröb

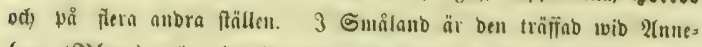
berg (\$3)m.), ocl) wio Berga ai Salmare län (Bett.); i D̃ilergölfi.

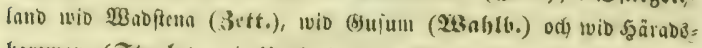

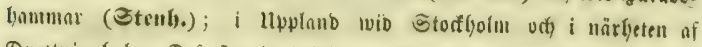

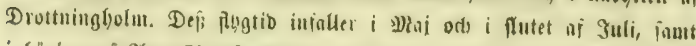
i börfan of 2 lugufti manaber.

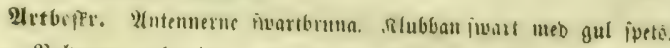
Balwerne gulagtiga med fwarta ftrimutot od) untertill meb

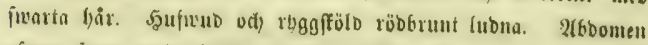
ofwan fiunrt mco röbbruma atomer, unbertill fuartgrâ. B Böftet jumattgrått, libet. Fötterne juarta meb gulagtiga tibier od? tnries. Wingarne i utanten tondabe, huarbera förfebda ued en gröfre tand. De finfre ofiwan länge abbomen felwurnn méb röofruna, linga b) ตำ.

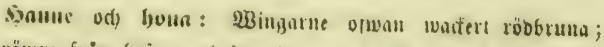

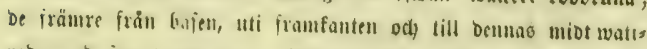
rabe med juats od gult. 3 jamun faut fai twestue, inait mes eller minbre inetīiga, jwarta Räcar; emellan bida beşa funnes

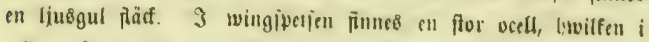

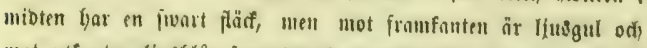
mot utfanten limsbla, fant bet begränfab af ett funall, fwart 


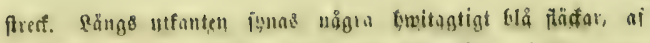

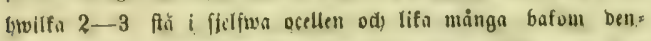
fnmma. I lafningarnes främre frörn finnes äjuen en for ocell, fom $i$ mibten är firart od lluabli, ment utomfring oms giitwell of ell lywitgri sing, junt inåt begrànfas af fwart. Lins bertill äro allo rbingarne fwarta, wattrabe meb folimarta fmås flieff, od bfwer biffen af bnfwingarme gå twenne varallela, toå giga twerlinier of fanuma färg.

Sarven är enfärgabt jwart med många byita punfter od

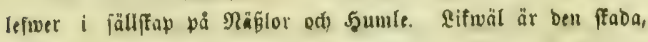
four ben affabfommer, uteıbdiy, emeban ben fällan förefommer i mängo hos oṕ. \$uqaı är grönagtigt affegrâ eller brunag. tig mob fincua taggar ods fpetfar, od en twabelad ftiertioets, iamt prood meb be imanlign gulbs efler fllimeriärgabe flädarne.

\section{Trib. Heliconides. Borso.}

Pedes 6 omnes yressorii, invicon conformes, fero requales.

Cellula discoidalis alarum posticarum costa transversa, ceteris xuguali, clausa. Collula anticarum costa transicesa aut conlinua, aut plus minus abrupta clausa.

Margo anticus alarum anticarum costis tribus of ramulis duobus

1. fantum uno quartac, rarius costa tantum unica of ramulis quituor socundx, oxtendifur.

Costa alarum fosticarum aut 8 aut 9: 1:ma aul e trunco communi cum 2-4, ant o basi separatim, semper tamen radire simplice egrediens at ramulum ant nullam aut distinrtum in Jobun basalem mmittens. Cum costa sunt 8, seplima et aclava o basi, sed cum 9, sunt, octara ot nona o basi egredimulur. Coste anticarum 8 t. 9, ad basin inler sa reguales, sexta vario modo, ramosia dit 1:ma, o hasi oprediens.

Ale postice pro recrptiono abdominis canaliculate. 
Larra cylindrica I. elongata, inermis, ghabra I. sericea.

Pupa griblosa, antieo unimucronala, moda, ano et bilo per medium transverso alligalia.

Fotterue: afla 6 gångiötter, findemellan lifformiga, năftan lifa långa.

Difffïltet på bafuingarne flutef getom en twenterb, jom år lifu be öfrige wingnerverne. De iröแreह biffiâlt snting fon flutet genom en jullftänoig, cllet genom en mer eller minbre aibruten twernerb.

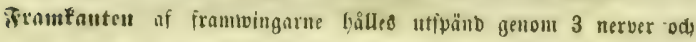
odi) 2:ne grenar eller blutt en enda of en $4:$ be, iällan genom blott en ellba nerb od 4:ra grenar af ent anbra.

Sierverue på bafningarne antingen $\&$ filer 9: bell 1:fta utgåenbe antingen fran gemeniam ftam meb ben 2:bra-4:be eller of

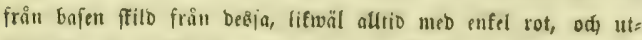
fänbanbe ait rotloten antingen ingen eller off en thblig gren.

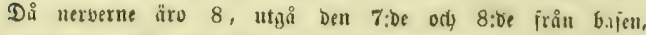
men bå be äro 9, utgî den 8:be ods 9:oe från bajen. Fraut=

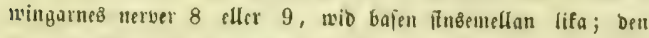
6:te pỉ olifa iätt grentig od den 1:fta แţående från bajen.

şafivingatuc för aboomenz upptagande biloande en ränแa mebelf inbre fautens nebföfning.

Earycu cylinbriff efler nflung, obewäpnad, flit eller filfegluben.

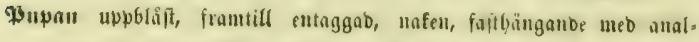
taggen od mebelft en omfring mibten flagen tråb.

De till benna grupp Görande arter lajima iumliga en fuabe or itrallanbe, andra beremot en flabdrande ody affruten fingt. Nagra äro ganffa ftugga oú) liwåra at länga, anbra decermot iffe. Störita

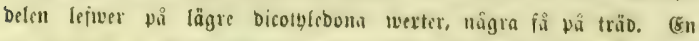
bel förorfafar uti träbgatroar temligen for ffada, ipmerhet jom besia arter äro ganffa allmämma. Färgen bo fiarilurme âr imbafuelgul eller lywit, obetwoligt blandabt me ịvars.

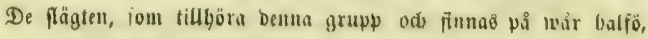
funna på follanbe fät farafteriferas : 
Costa prima alarum posticarum simplex, ramulum

A. nullum in lobum basalem emittens.

Förfta nerven på bafwingarne enfel, ei utjänbunbe någun gren ait rotloben.

, Costæ alarum anticarum 8; 6:ta qua-

1. $\left\{\begin{array}{l}\text { driramosa. } \\ \text { Frammingarnes nerber } 8 \text {; ben } 6: t e \text { fot }=\end{array}\right.$ Colias. grenig.

Costæ alarum anticarum $9 ; 6:$ ta tri- ;

2. $\begin{aligned} & \text { Frammingarnes nerver } 9 \text {; ben } 6: \text { te tres } \\ & \text { grenig. }\end{aligned}$ Goniopteryx.

costa prima alarum posticarum ramulum in lobum

B. basalem emiltens.

Förfta nerven på Gafwingarne utjänbanbe en gren it rote loben.

Costa 2;da alarum posticarum hira-

1. $\left\{\begin{array}{r}\text { 2:bra nerben på bafwingarme twågre }= \\ \text { nig. (Frammingarnes nerver } 8 ; \text { ben }\end{array}\right\}$ Uetcophasia.

mosa. (Coste al. anticarum 8 ;

7:be femgrenig).

2. $\left\{\begin{array}{l}\text { Costa 2:da alarum posticarum simplex. } \\ \text { 2:bra neroen på bafwingarne entel. }\end{array}\right.$

Costx alarum anlicarum $9 ; 6: 1 a$

1) $\left\{\begin{array}{l}\text { quadriramosa. } \\ \text { Frammingarnes nerver } 9 \text {; ben 6:te } \\ \text { forgrenig. }\end{array}\right.$

2) Costx alarum anlicarum 9 ; 6:ta biramosa.) Pieris.
(Frambingarneo nerber 9 ; ben $6:$ te twăgrenig.

3) $\left\{\begin{array}{l}\text { Costx alarum anticarum } 9 ; 6: \text { ta triramosa. } \\ \text { Erammingarnes nerber } 9 \text {; Den 6:te tregrenig. }\end{array}\right\}$ Aporia. 
Slägtet: Coliass. PABr, et Nutr.

\section{(Ganoris Dalm.)}

Antenua broves (tertiam partom marginis antorioris alarum anlicarum vix superantes), rectx, apicom versus incrassatæ. Clava olsconica, cylindrica, haud doterminala, apice truncata, obtusa.

Caput magnum, thoraci fore tequale, pilis densis conformitor hirsutum.

Palpi compressi, porrocti, subdivorgentos, intus subnudi, in latore exteriori lamon squamali; infra listi, pilis longioribus divaricatis instructi : iriarticulati. Arliculus basalis medio brevior, fere reclus, arliculationem vorsus nonihil attenuatus; medius basalem ultimumgue Ionģitudino suporans, conformis; ultimus minutissimus, tertiam partem articuli basalis non superans, obovatus apice rotundato.

\section{Oculi nudi.}

Pedes invicen conformes; lemora in/ra pilosa; tibix, praserlum posticorum, dentibus brevihus instructæ, sed intermediorum et posticorum ad insertion'm tarsorum calcaribus duohus validioribus etiam armate: tarsi etian brevo denlati; articulus primu farsorum longi simus, reliģuis fero xqualis; 2:dus tortio, hic tamon quarto, minutissimo, nonibil longior; b:tus tertio requalis.

Alae intogra.

Costro alarum posticarum 9: 1-4 o trunco communi supuriori, 57 o trunco communi interiori of 8-9 o basi egredienI's; prima simplex, ramulum nullum in lobum basaleon emiltons; socunda arcuata. Costæ alarum anticarum 8: 1:ma e basi, 2- 4 o trunco communi inforiori, 5 a costa transporsa cellulae, 6-7 a trunco communi superiori ot 8 e basi egredientes; 6:ta quadriramosa. Costa transversa restulae alarum anticartum intor costam 4 ut 5 modico arcuata. 
Intenneruc forta (fnapt längre in trebje belen of fromuringors:ce fräure fant), เăts, mot fapetien tilltilodnabe. Stutban omuränbt

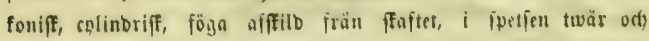
trubbig.

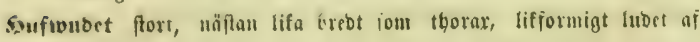
tătftåenbe bi̊r.

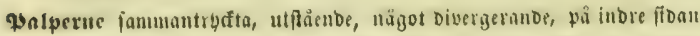

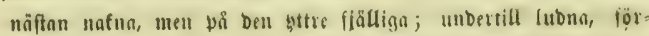
febba meb längre utfperrade hair ; trelebnde: Vaobleben fortare ä ben meblerfta, näftun răt, mot lebiugningen något afjmalnanoc;

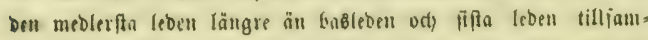
mantagna, lifformig; Den faffn leden minft, idfe längre än trebir

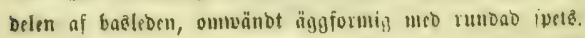

\section{Onouen natna.}

Föttern fingemeldan lifformign; lärn undertill hisiga; tibierne, ifgunerbet baffütternes, förjebon muco forta tänder, men mellanföto

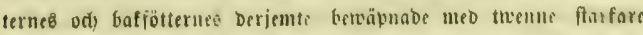
iporrar wio sarferme lebfogning turforme diresen tandade; 1:fla targleoen ganfta lång, näftan lifa lăng med alla be öfriga; 2:Dro leden taingre äı Den 3:Die, od) bemue längre äı ben 4:De, burilfen är minft; 5:te lifa lång meb 3:ble.

Tiingarue belorảboade.

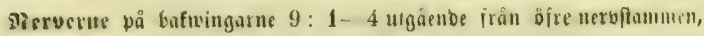
5-7 frân nebre neroftamumen odh 8-9 irản bajen; den 1 :ftn enfel, utfänbande ingen gien åt cotluben; Den 2:bra fröft. Eram.

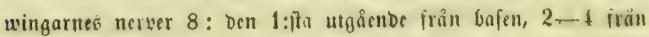
mebre necuptammen, 5:te frân ciaffältets metuerv, $6-7$ frän

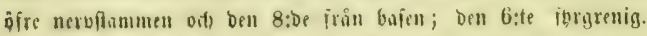
Fwernersen wid ramaningarmes bifffätt nảgor büld cmellan 4:be od) b:te wingnerberne.

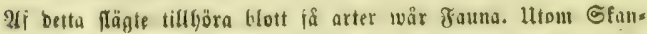
Dinquien är bet talrifore reproienterabt. 2arterme förebraga ffogertrafter

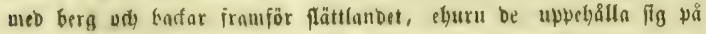

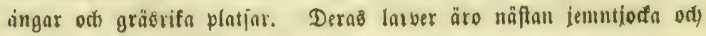


Femuxna meb matet forta bair, ia att be lajma utfeetibe ai utt wara nafna. Bupan är på mibten lywälfo ợ förfebo meb en upphölo egg. Fiårilarne förefomma nuerenbels meb blott on generation on siret.

\section{Svafvelgula Mófjärilen (Colias palæno Lin.).}

Alis supra sulphureis aut athidis, apice lato niygris limbrisque rosei; posticis infra flatidis atomis nigris adspersis, macula simplici centrali argentea. L. al. exp. 50-57 millim.

Wingarme opwan frofuelgula eller broitagtiga, $i$ fpetien med breb finart borba od) franfarne rofenfärgabe; be bafre unbertill gul. agtiga, beftröbba meb täta fwarta atonter od fôtjeboe ateo en enfel

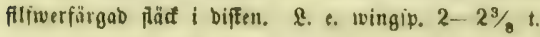

Syn. Pap. palano Lir. I., Sv. 10\&1. Mabr. E. S. III. 1. 207. 648. Ilibn. Toxt. 67. 21. Colias. Ocbs. Schm. I. 2. 18\%. IY. 32. et 156, 157. Zeti. Ins, kapp. 908. 1. God. et Dup. Lepid. II. 3\%. pl. 4. 1. 2. II-S. 'Text. I. 103. Ganoris Dalm. 1’ap. Sv. 89. 9. Pap. europomene llübu. Pap. 1. 86. 1. 4344. 43\%. Pap. philomene Ilühn. Pap. 1. 117. 1. 602, 603, ot fig. 740. 741. Colias werdandi II-S. Pap. I, 83. f, 403. 404. I. 8. S. 41. 42.

Obs. Col. werdandi $\mathbf{I}-\mathbf{S}$. ost a Col. werdandi Lett, Ins, Lapp. 908. \&. certisuime longe divorsa. Cum speciminibus lapponicis, ad Col. palanonem pertinentibus, bens congruit et rariotatem haud memorabilem, nullo modo speciem distinctan constituit.

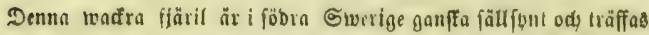
nigon gaing blott i fogatrafterme, Så har lag iunnit ben $i$ Sfäne tvib 3imetofin od 202 . Wramb od) Bror. Bohemnu bar träffat ben wio

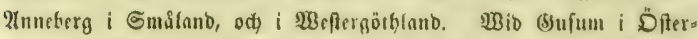

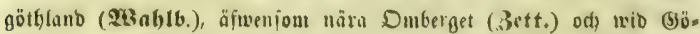
theborg (2Bablb.), förefommer ben of, men temligen fpariant. Llti meblerfia odj norra Siveriges bernigare lanbffuper är bent beremot allmännari, fåiou wio Rorberg i Wefintanlanb (Frobridi), nio

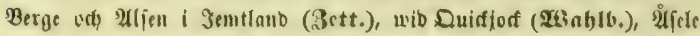




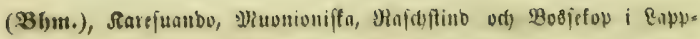

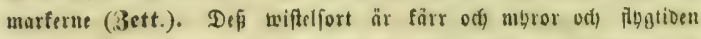

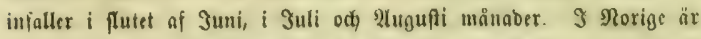

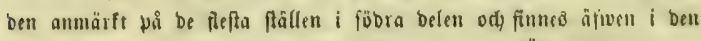
norra. WBib Cebriftiania, Solber, i Mingerige od) D̈fterbulen är ben fumuen (उicbf:) och) innes ber twara näftan allmänutare än $i$ Swerige.

Mrtbeffr. 2(ntenuerue röbugtiga, unbertill ftölande $i$ gult. Stub. Gan 1 ipetien unbertill röbgul. Balperne Guvitgulagtigs lubna.

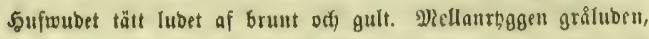
bos fannen fiotanbe $i$ gult. 3)t6bomen ofiran firart meb gult puber, undertifl hos bamen gulagtig, hos fonlan fwritagtig. gröftet hoo ben förre gulelubet, god ben fednate grilubert. Fötterne boa den förre gula, yoo den febnare grå.

Sanue. Wingarne ofwan lwajwelgula med fwart, năgot gulpubrab, inåt wågig borba, fou på be främe är Grebalc of

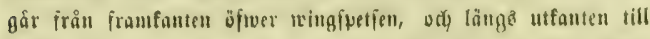
infanten, lywilfen ben useb enl funal hif föler ett fort phofe; men på buftwingarne är borban jmalare, lifaleres inà mågig, nåenbe från framfansen od ufwer cräme börnet till mibten af

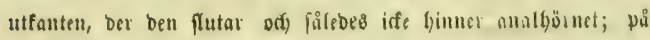
alla wingarme ä: borban lemnt baggormiß od) brebaft $\mathrm{i}$ winge fpeffurme. 3 biffen af frumbingarne finnes en mer eller minbore toblig, fwast tuwerpäa od) pî jauma ftälle ai boftwingarne en misbre gut unformig, runbad efler fantig färf. Sha unore poun àro twingarne gula; be fräntre längs fromfanten ods äfwen $i$ biffen närmare winglvetien, famt be bafre öfwer lefa btan fint brunpubrabe. 3 diffen bajian be främre en twerftienbe fwart fäă, jom $i$ ntiolen är gul, od be Gafre pä iamma fälle en

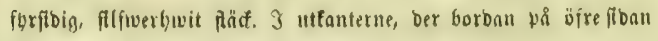
gåx fram, äro wingarme på unore fiban gula meb fmag, grön. agtig anftrufning. Fronjarne äro rofenfärgnde. 
Syouan hat unilgarne på ofre ftban bwita, ben fwatta bors

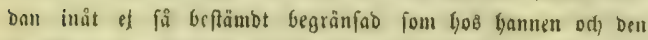
upvför på framuingarne $i$ Gafre Görnet utan att följa infinten,

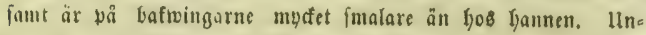
Dertill äro franmingarne lytwita mé guln fpetfar och be Gafre gula, Fo̊r üfrigt lifnar bon bannen.

Earven år gröl od) gulftrimmig, famt fwart punfterab. Den ffall lefwa bi Coronilla, in fiellewext, fom ber od hyar iörefommer, men utan triffwel tillgriper ben äfwen anbra wexter.

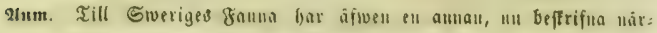

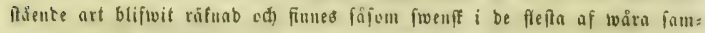

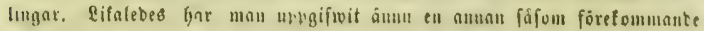

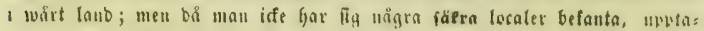
gas endâ̊ vo̊ betto fátt båtas beftrifuingar:

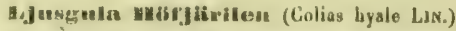

Alis supra sulphureis 1, albidis, anticis macula media discoidali maeulıque apicis nigris; posticis macula fulra, infra macula sesquialtera artenlea, ferrugineo circumcincta. $I_{s}$ al, exp. $46-4$ millim.

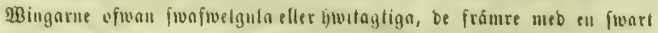

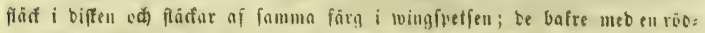

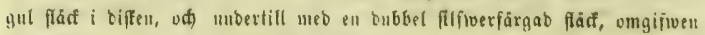
af róbbrunt. \&. e. wingfiv. $1 \frac{1}{8}-1^{16 / 30}$ t.

Sỳn. Pap, hyale Lin. l', Sy, 1040. Fabr, E. S. III, 1, 207. 649. Hobn, T'ext. 67. 19. Colias 0gus. Schm, I. 2. 181. IV. 32. Tneitsci. X. 1,93. GoD, el Dup, Lepid, I. 46, pl. 2. secund, f. 2. II-S. Text. I. 104. 1. 6. f. 33. 34. Gaxonis DALA, Pap. Sr. 89. 8. Pap. paleno IloBN. Pap. ᄂ. 87. f. 438. 439.

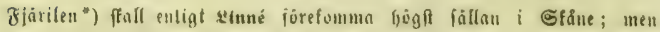
bettu ăr treligen ett miptan. Golligt Doct. Solmosins unvgift $i$ bref till

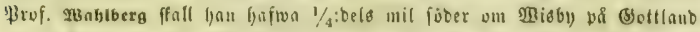
rell 2 September fir 1829 fingat temm art. Eremplaret, fom förwara

") In Cottlandia habitare fertur. T'estimonia ulteriora tamen desiderantur. In Scania non occurrit, quantum mibi innotuit. 
1 Grci. Mablberge nipilba famling, ed fom af Rolmobin ubfgifwes wara

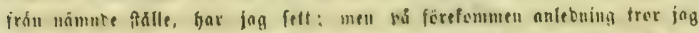

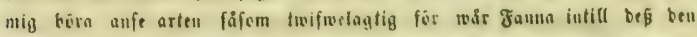
Slifwer soibare efterforfab.

Mrtbefer. Autenuerue robagtige. Rlubban I fuetfen gul. Rrvbubelars nes färg för ifrigt fáfom hod forregåenbe.

sanne. Wisgarne ofman liuft frofmelgula, wib bofen fwartpus srabe; re främre med en runtao fwart flä i bifell och ett fwart banb if.

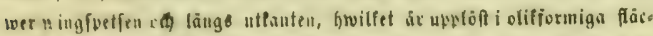
far, fí att fläfar ai grunbfóggen beraf iuneflutab. Baftuingarne meb en

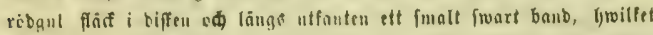

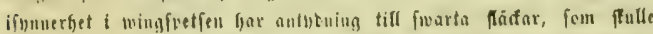
iunefluta anbra of grunbfärgen. Mubertifl aro frombiugarne ljusguta, i fretfen böggula, wib bafen ent) längs framfauten fut fwartpubrabe, od

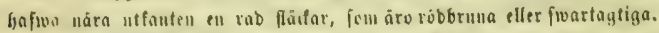
Bafmingarne äw wif fammn fion förgula, frin bofen ad) ufofwer mibten

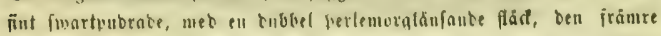

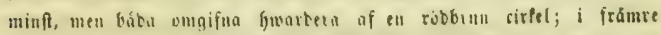

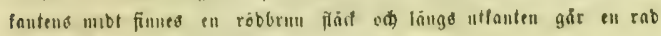
bylifa. granfarue tofenfärgabe.

\$̧onan lifnar lganen, men bar wingarmes ofre ud frambingarned unbre fiba givitagtig, obelyoligt braganbe i gult.

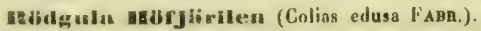

Alis supra fulris margine t.igro, (fetninw fulro maculato); anticis inacula discoidali i igra; infra omnibus virescentibus, anticis macula discnidali nigra, posticis macula punctnque adjacente argenteis. L. al. exp. 50-56 millim.

Bingarne ofman robgulu met fwart (Gus honan gulfästig) borba; be frảmre mé en jwart flás ! biffen; afla untertill grơnagtiga, be främre meb ell imart bifflad on be bafre meb en verfemorfárgab flad od ber brebuib liganbe punft. g. e. wingiv. $2-2 \%$ t.

Syo. PAP. edusa Fabr. I. S. III. 4. 206. 643. Ilobs. 'Text. 66. 17. Colias Ocis, Schm. I. 2, 173, IV, 32, et 136. TREitsch, X. 1. 81. PaP. hyale lloBs. Pap. t. 85. f. 429-431.

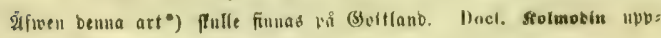

") In Gottlandia habitare fertur. Tentimonin nlerioria lamen desiderantur. 


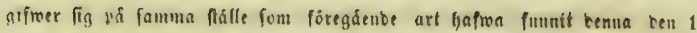
(d) kell 22 Sept. 1829, sd) fetau fera gånger. Sgäba exemplaten, banue

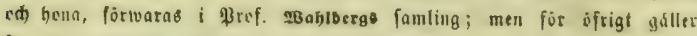
fammu sumårfning om beuna fom om fôrra art.

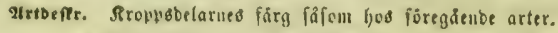

Sasıe. Mingarue ofluau robgula; be bafre foutanbe $i$ grent va) fiut uorfunbrate; meb en fwart borba ( - af famma form fou bos iunfwefgula 50 fjärilen --) Gnilfen genumbrages af be robgula winguers veruc. Waib Diffältets flut wi fromuvingarne finues en zunbab fibart flä

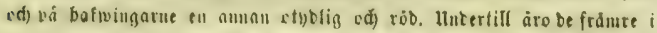
riften orangegulu, i framfanten, fem as froctonotab, od i mingfuetien

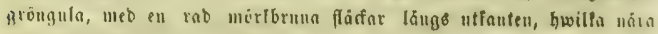
infanten blifwa fmarta. S3afwingarne áre grénagfigt gula meb ell fture od ell brebwibliggnute minbre berlumorglänante flä i ciffell, sungifuen af en robbrun linia, fant Idngo utfanten en rab robbruma flacar. Franjarne fafom bos föregåenbe.

sonan, mörfare röbgut ảu banuen cơ bó bafivingarne fótante $i$ brunt, hat gulfactig berba i utfanten ed tyoligare rob fikd i bais wingarues bift. Unbertill âr hou lifalebes mörfare äu Gannen, Gwilfen hou for eftigt lifunr.

\section{Hordislka Möfjärilen (Colias Bookhii Boss).}

Alis supra fulvo-lutois limbo communi nigro, flavo pulserato; anticis ad costam transversam collule lineola nigra, infra in modio alba; posticis infra obscure fulvo-virescentibus, donsy atro-pulvoratis, macula argontea, intordum gemina, in disco. L. al. exp. $40-45$ millim.

Wingarne ofwan röbagtigł gula meb gemenfam fwart ods gul. unbrab borba; be främre meb ett freart, unbertill $i$ nibten lywitt ftred wib biffälteto twermerv; be Gafre unbertill mỏrft gulgrüna, ofmerftröboa med tätn fwarta atomer od i biften förieboa meo en

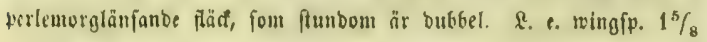
$-113 / 10 \mathrm{t}$.

Syn. Colias Boothii Ross: Appondix to the Narrulive of a second voyage in Search of a North-Wost-l'as-ist: (1835) p. 65. n. 10. pl. 4. a. f. 3-5. Col. liecla 
Lefeb, Act. Soc. Ent. Gall. 1836, p. 384. 1. 9. f. 3. 4. Zett. Ins. Lapp. 908. 3. Col. Boothii II-S. pl. 96. I. 459. 460 .

Ohs, Preter liguris jau citatas habot Herrich Schäffer alias Col. Boothii in $\{\mathrm{ab}, 8$, f. 39 ot 40 . Figuræe illa ad hane speciem pertinere non possunt, quia limbum nigrum multo tonuiorem et seriem, otsi obsololam, macularum fuscarum ostondunt. P’ossibile ost ut figure monoratx ad Col. chionen Ross 1. c., speciem mihi ignotam, portineant.

Demn Gögnordifa fläril, builfen un för förfîn gången beftrif* เves fåfom froenff, upptädtes i Roppmarten of wair förtjenfifule (5n= tomolog, łirof. 23obeman, lwilfen fam den $i$ en bal emellan Duif,

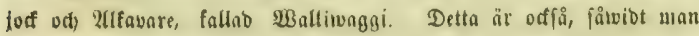
bittills fännet, enba lofalen för oenjanıma inom Sfanbinabien.

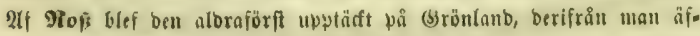
wen | Gmenfta famlingar eger exemplar.

2lrtbefr. Intennerne farntofinfärgade. Stubban ofivan brunag. tig, $i$ fpetien od, unbertill röbgul. \$alperne gulagtiga, unbers

-) Descr. Antenne enceinear. Clasa supra fasca, apice el parto inferiori fulva. Palpi favi, infra cineren-rufo pilosi. Caput et thoras pilis densis albidis, in capite et collasi in roseun verteatibus. Abdomen supra pilis rarioribus albidis, infra flavidum. P'ectus cinereo pilosum. P'edes cinereo-Juteis.

Mas. Alwo supra lulvo-lutew linbo lato nigro, flavo pulveratn, communi, in posticis tamen attenunto ot in medio marginis exterioris desinente. Ad basin suut alæ omnes punctulis nigris adspersa, qua, abdomen sequentes, se supra discuu extudunt, ubi etian macula adest subinconspicua fulva. Ad finem cellulo alarum anticarum linea transversa utrinque nigra, qne ioterdum supra in medio macula flava, semper tamen infra nacula alba est instructa, abservatur. In limbo nigro lineola flaro, e costis alarum excurrentihus orten, adsunt. Alm anticm infra fulvo, ad maryinem anteriorem et in apice nigro punctata, ad angulum posticum maculis nonulis fuscis prope marginem exteriorem cincreum ornato. Aso postico inlra obscure fulvo-virescentes, ad basin densius, quam ad mnrginem exteriorem, atro pulveratx. Ad cellulom discoidalem macula argentea, interdum gemina, prosertim postice brunneo marginata, conopi- 


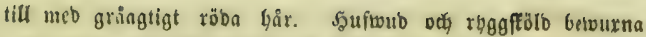
med Juvitagtiga T:år, Gyilfa på bufwubet och halsfragen ftôta $i$ rofenröbt. 2)(6oo:tren ofwan meb fineitagtiga glefare bår, unber. tifl gulagtig. sBröftet gulgri̊tt, lubes. Fötterne gulgrå.

Sonme. Wingarue ofman röbgulagrigs gula med en teme ligen breb, fwatt och gulpubrab forba $i$ utfonterme, Grilfen på bafuingarne afiunalnande upphör twio mibten. WBid bafen äro svingarne beftrobboa meb iwarta atomer, lywilfa på be Gafre gå längB abbomen utåt biffetl, font probbes af en otyblig röbgul

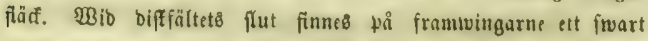
tweeffired, bwilfet flunbom ä i mibten ofwan förfeot meo en gul fläf, men slltib unbertil! bar en lyvit. Wingnerverne utgå i utfanten med juala, gula frecf. Unbertila äro be främre wingarne rŏbgula, länģ främre fanten od, twers of piwer biffen lant $i$ wirgipetien pubrabe med frart, ods rvib inbre fürnet förfebba meb några jwartagliga fäafar nära ben gråagtiga ut. fanten. Bafisingarne äro undertill gulgröna, tätt öjwerpubrabe

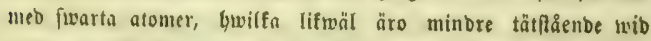

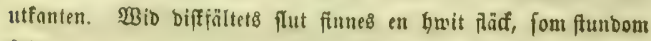

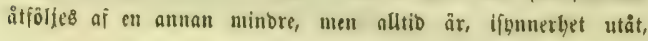

citur, ut etiam ad medium marginis anterioris macula fusca, o qua series arcuata macularum ejusdem coloris, discum obscuriorem a marpine dilutiori descernens, ad angulum ani ogreditur. Fimbrim roses, supra in flavedinem vertentes.

Femina, quan non vidi, limbum in pagina superiore alarum latiorem, maculis flavis ornatum, el alas posticas supra omnino nigro pulveratas, inlra obscuriores quam in mare gevere fertur.

Ilab. i Lapponia rarissime. Celeberr. Dum. Boheman inter Quickjock et Alkavare hane speciem quam maxime borealem in Scandinaria detexit.

Obs. Specimina e Groenlandia orla, in parte inferiori alarum postiearum ad maculam argenteam discoidalem el pone illan halsent in culam rufo-brunneam, in muerone acuin postice excurrentem, qua omnino carent sueoana, 


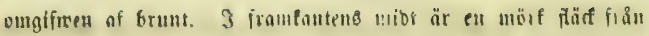
luvilfen funbou en böjo tub af anbua bulifa gàt till anallöre net, ftiljanbe ben mörfare biffen frin ben lfufare utfanten. Franiarne voienfärgabe, oftunn ftötanbe $i$ gult.

Sounn fall Mafiwa $i$ utfantes af wingarne oiman brebare

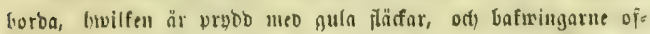
man left ods ballet öfiverpubrabe meb fwark atomer ods un=

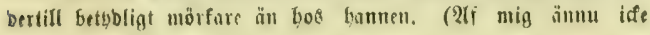
jebb).

\section{Caringula Hajtjärilew (Colias nasles lioisl.).}

Ali Inaris supra viresconli-sulphurois, lomina albido-llavosenntibus, limbo communi maculari nigro; anticis macula discoidali nigra; posticis infra obscure vireseenti-flavis, denso nigro putvorafis marula simptici argontea in modio. I. al. exp. 15. 50 millim.

Wingarne ofwan bos Ganmen grönagtigt fwajwelgula, bos bo:

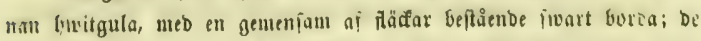
främre med ell flwart lảd $i$ biffen; be bafre unbertill mörft grön= gula mco tätfaienbe fwarta atomer odi $i$ mibten en werlemorglänjanbe, enfel đasc. $\mathfrak{l}$. e. twingfp. $1^{13 / 16}-2$ \&.

Syn. Collas nas/es Boisd. Icon. 1. 245 . pl. 8. 1. 4, 5. (figura finoad colore incorneta). H-S. Toxt. I. 103. pl. 7. 1. 37. 38. pl. 83. r. 401. 402. (figura optimæ). fiod. et Dup. Lepid. Suppl. 1. 98. pl. 15. f. 4. b. (lip. quoad colore incorructe). Col werdandi Schlirr. Leti. Ins. Lapp. 908. 2. (corte).

obs. Col. werdandi Kett. ost C. nastes Bolsd. ut o spocimine originario, in collectiono schönherri asservato of a mo viso, satis apparet. Cur Cotuberr. Dom. Zetterstedt specimon summ ad C. mas/en Boisd. non rotuli, facile porspicimus. Figur nompo Boisdovali ul oliam Duponcheli spociminibus suocanis quoad colorn non oxacte quadrant.

(\$)öngula \$öriärilen är lifjum föregåcnbe en bögnotbiff art. Den fösefommer Glanb Rapyfta ficllarne, oer ben duger offwer nufna platiar mellan fröbrifmorme. Enligt ßrof. Butterfeot är Den 


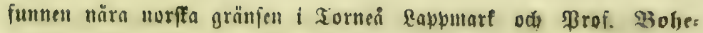
man Gar bemfört ben från ell fjellbal, wib namn SBalliwaggi, belï* gen emellan Duidforf od, 2lifomare $i$ iammia Lappmarf.

2Artbefre. *) 2(ntennerne röbagtiga. SRuthan ofwan brun, unbertill röbgul. \$aIperne fwafuelgula, unbertifl bewuxnn meb rôsagtiga, tätffånbe bår. Siroppen fwart meb bnitngtig lubo, iom på

*) Descr. Antenum ubrenernles. Clava supra fusco, infra fulva. Palpi sulphurej, iufra dense fulvo 1. rubro hirti. Corpus nigrum pilis cinerco-albis, in sapite "t rollati at roseum verlentibur. Pectus flavohirtum. Pedev rubri.

Has. Als supra sulphurea, ohsolete virescentes, punctulis nipris, prasertim ad cosins, ad basin, ad marginem anteriorem ularum anticarum et ad murginen isteriorem alarum posticarum densis. In margine exteriori alarum cat limbus flavo-pulicratus niger, qui in inedio marginis alarum posticarum desinet el deinde punctulis acerratis nifris tantum indicalur. I limbo memoralo alarum antiearum macula adsunt $6-7$ ovalø, flava, quarum plurima lineas tenues flavas in ipsam marginem emiltent. Macule ejusmodi in limbo alarum postirasum, minus tanen destinctio (interdum nullo), reperiuntur. Ad rellulam linea transversa nigra in anticis et macula suhinconspicua in posticis locum tenet. Mas antica infra sunt pallide flaro, punctulis densis nigris ad marpinen auteriorem et exteriorem et in apice obsilæ; margo exterior in colore cincreo maculas gerel pallide flavas, maculis pagino superioris respondentes et intus serie maculafa, plus minus continua, el punctulis acervatis nigris formata, lerminatas. Linea niz̧ra discoidalis (sæpe nulla) interdum argenten pupillata. Ala postics infra obscure virescenti-flara, dense nigro pulverata, ad marginrm exteriorem dilutiores; ad cellulam thiscoidalem macula argentea, rufo-brunneo prosertim postice terminata; in medio marginis anterioris macula infuscala et interdum series maculata cjustem coloris inter enlorem obscuriorem disci et dilutiorem marginis exterioris. Fiubria rosex.

Femina mari est simillima, sed albido-llavescens olsoletius virescens et maculam in parte inferiori alarum posticarum discoidalem plerumque geret majorem.

Hab. all latera alpium Lapponias I'arius, ubi papilio in locis sterilibus, nive nou plane obrutis, circumvolat. Hane specien Celeberr, Dou. Boheman inter Quickjock et Alkaware Lapponia Tornensis eliam enl!eghit. 
bufwud od, halsfrage pöter i vofrinobs. Bröftet gulstubet. götterne röbs.

Sanuc. WBingarne ofwan jwafmelguta, fungt fätande : nrönt, beftröboa meo fwarta atomer, builfa ifpunerbet bopa ftg omfring iringnerverne, wib finjen, od längs framfanten af frams wingarne faut inore bälften af baftvingarme. \&ängs utfanterne gîr ell pwartagtig, gulpubrao Gorba, fou pi bafwingarme upplür wib niloten od) berefter blott anthbes genom fupabe iwarta ato= แแr. 3 benua forba finnas yå framuingarne $6-7$ oyala, gula

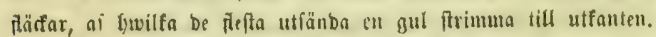

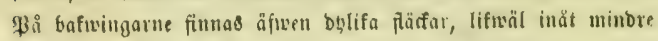

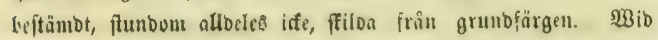
diffältets fut funes pai frumusingarne ent iwartagtigt tmerifterf,

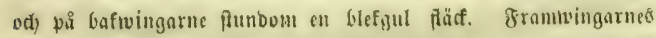
unbre fioa är blefgul mco täta, fiwarlagtign ntomer längs from. fanten od utfanten fant twere ö öwer wingipesion; ufonten ät

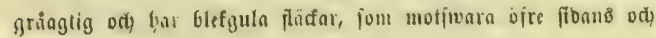
inåt begränjas nf en mer eller misibre fammanlyänganbe rab aj mora fläfar, bilbade genom flopabe iwartagtiga atomer. Diff: jältets fwarta farest, fom dit od) Dă jafunes, yar ftumbom en Guritagtig \$upill. Bafruingarne äro umbertill mürft gröngula,

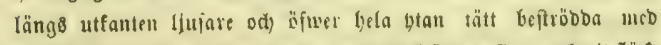
iwartagtiga atomer, famt lyajwa wio biftiältets flut ell lywit fläd,

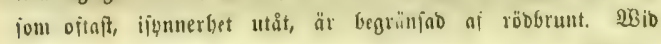
midten of framfanten är en mörf flärf od frundom en rab $D(s)=$ lifı $i$ iärggränien mellan ben ljuiare utfanten on mörfare biffen. Franjarne rofenröba.

Sounu lifuar lyaunen, men bat reingarnos ofise od be främres undere fiton fowitgul, tögft obethbligt flöknbe i grünt.

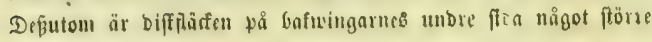
än bos hannen. 


\section{Slïgtet : Goniopteryx. LAaci.}

(Colias Fabr. Latr. Ochs. Zett. Ganoris Dalm.

\section{Rhodocera Boisp.).}

Autenna ut in genero qqrecedonto brevos, sed deorsum arcuato, versus apicom sonsim incrassate. Clava obconica, intus complanata, haud doterminata, apico rolundato, mucrono minutissimo inflexo instructo.

Caput parvum, thoraco minus, crista orecta acuminala ox pilis densis inter oculos ot sub antennis instructum.

Palpi compressi, vix divergentes, squamati, infra dense pilosi apice subnudo, triarticulati. Articulus basalis reliquis conjunctis longitudino aqualis, arcuatus, articulationem versus allenuatus; medius uttimo longior, oblongo-ovatus; ultimus minulissimus, subconicus (apicom versus altenuatus apice acuminato).

Ocull nudi.

Pedes intor se conformos; fomora infra donso pilosa; tibiø tarsique inermes; tibiæ tantum quatuor posticorum dentibus duobus brovilus ad insertionem tarsorum munilæ. Arliculus tarsorum primus tribus soguantibus conjunctis longitudine xqualis, secundus tertio, lic tamen quarto minuto longior, sod quintus secundo longitudino xqualis.

Alax ingulato-dentatx.

Coste alarum posticarum 9 omnino ut in cenere precedonto sod socunda recta; anticarum 9: 1 o basi, 2-4 o trunco communi inferiori, 5 e costa transversa collul $x, 6-8$ o Irunco communi superiori, 9 o basi egrediontes ; 6 biramosa. Costa transversa collula alarum anticarum inter costam 4 ot 5 valde arcuala, convexitatem orga collulam vertens.

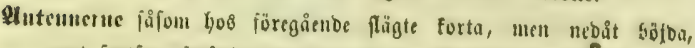
mot fpetien fuâningom tiltifoctuanbe. Slubbun omuånbt fonif,

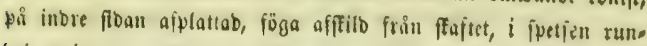
bab meb en ganfa liten, inbölo tagg. 
Sufwubet liset, mudet fmalare än thorax, mob en fpetfig och ups. rät af tâta bår filloab crifta emellan gogonen oft) unber an* tennerne.

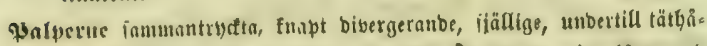
rige, I jpetien näftan nafna, trelebabe: Gasteden lifa lång mè be öfriģe tillammuntugne, 6öfo, mot leofogningen affmalnanbe; mellanteben längre än ben rifta, aflängt äggformig; ben fiffa

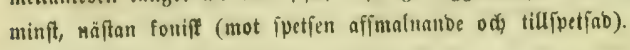

Sgouen nafna.

Fütterue finฮిemeilan lifiormiga, låren unbertill tät)åriga; tibierne

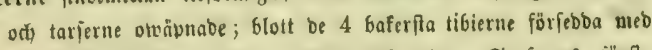
trestune forta tänber vio tarjens lebfogning. Tarfernes förfta lè lifa lang meb be 3:ne fölinno tillfanmantagne, ben 2:bra längre ain bin 3:ble, benne längre än ben 4:be, four är miuft, men ben 5 :te lifa lang neb ben anbra.

भBinnarue winfligt tambabe.

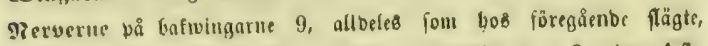
utom ben 2:bra, fom är rät; på frommuirgarne 9: ben 1:pata irån bafen, 2:bra-4:be fri̊n nebre nervitammen, ben 5:te från biffältet8 twernerv, ben 6:te-8:be från öre neruftanmuen oḍ ben 9: be från bofen; ben 6:te troågrenig. Difffältets twernerb pio irammingarne emellan $4:$ be od) $5: 1$ e mingnerberme mudet fröft, wändanbe fin convera fiba mot biffältet.

Detta fägte, bwaraf till wåa Fauna Gloft börer en art, laar larven näfan lifunmbe föregåmbe日, men ben âr bafåt afimalnnmbe

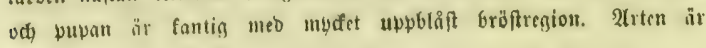

Citrongula 'costefjärilen (Gonioptor)x rhamni Lín.).

Alis maris citrinis, fomine flavo-albidis, unicoloribus, omnihus in medio macula minuta supra aurantiaca, infra Terruģinea. L. al. oxp. 55-60 millim.

W3ingarne hos Gannen citrongula, hos bonan gulburita, enfärgube, alla $i$ mioten med en liten päat, fom ofman ät orangegul, unber. till roftbrun. \&. e. twingip. $2 \frac{1}{4}-2 \frac{1}{2}$ \% 
Syn. Pap. rhamme din. F. Si. 1048. Falır. E. s. III. 1. 211. 261. Nübн. Pap. ᄂ. 88. 1. 442-4.4. Tox1. 37. 22. Colias Ochs. Selum. 1. 2. 186. IV, 32. Zett. Ins. Lapp. 909. 4. Forl. "I Dup. Lopid. I. 43. pl. 2. 1. 1. fanoris Dalu. Paj). Sv. 89. 10. Rhorlecera II-S. I'exI. I. 106.

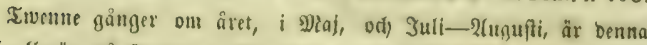
iiäril allmän wả ämzas, i ftogg̊lundar od träbgairbar utl föbra od mcblerfta delazme af Siwerige od Rorige. Şuru lyögt ban egentli. gen gå mot norben af waint lanb är of âmu obefant. 21 \$rof. Setteriteot unföres ban fäfom ganffa fälliunt $i$ fobra Rappmarferne.

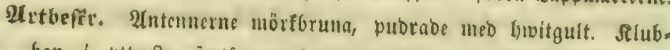
ban $i$ stterfta ipetfen gulagtig. Balperne längs öfre fionn

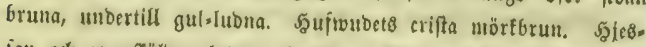
jan od) reggfföldent lubua af längre fllfwergrâ bår. 2(bbomen

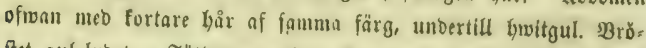
fet gulslubet. Fötterne gula. Şwardera wingen i guttre fan. ten med en grof tand, buvilfen på frammingatne finnes i wing= fpetfen, men tå fafwingarne $i$ fantens mibt. Für b́frigt äro wingarne belbräbbabe.

Sanute. Wingarme ofwan waxert citrongula, fwarbera ntcb en orangefärgab utinbre făc wio biffiältets flut; unbertill

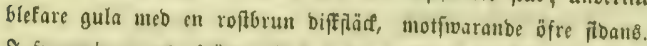
$\Im$ framwingnrnes främxc fant famt cmellan wingnerverns, nära

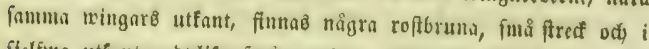

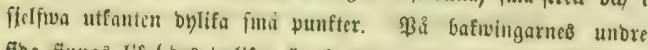
fiba finnas lifalebes bylifa ftrect vă punfter nära ody uti ut fanten; rotloben är i främre fanten merenbelo fiwartagtig.

Sounแ lifnar bannen, men ลี่ fåmäl yâ wingarnes dịe font unore fioa Givitgul.

Earveı är llufare cller morfare grŏn, gleşărig, med en

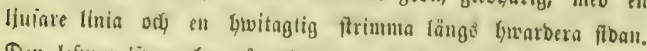
Den lefwer ijegnerbet på Rhamnus Irangula, men träffaß äfo

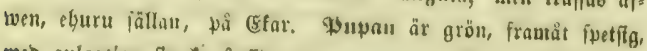
med gulagtiga firect pa floorne. 
Sliagtet : Hewcophasia. Sтвн. H-S.

(Pieris Larr. Pontia Fabr. Ochs. Ganoris Dakm.)

Antenus modiocres, distincte clavatæ. Clava obovata, brovis, determinata, compressa.

Caput magnum, thorace fore xquale, crista ex pilis longis orecta, divisa, inter oculos el sub antennis instructum.

Palpi compressi, porrecti, divergentes, squamati, triarticulati. Articulus basalis maximus, reliquis conjunctis multo longior, in medio dilatatus, articulationem versus sensim altenuatus, foro roctus, infra pilis longis divaricatis, articulos roliquos fero occultantibus, instructus; medius ot ultimus minutissimi, haud tertiam partem articuli basalis conjunctim superantes, ovati, modius tamen ultimo nonibil longior.

Oculi nudi.

Peles inter se conformes; fomora infra parcius pilosa; tibiæ tarsique inermes; illæ unguibus nullis ad articulationow tarsi munitæ. Articuli tarsorum 1-4 fere æquales, quintus minimus.

Alæ integræ, lanceolatæ. Collula alarum minuta, tertiam partom alæ non occupans.

Costa alarum posticarum 8: 1 e basi, secunda biramosa e trunco superioro, 3-6 o trunco inferiore, $7-8$ o basi egrediontos; prima ramulum bimucronatum in lobum basalem omittens. Costæ anticarum 8: 1 o basi, 2-4 o trunco communi inferiori, 5 o costa transversa cellulæ, 6-7 o trunco communi superiori, 8 iterum o basi egrediontes; 7:ma quinqueramosa. Costa transversa anticarum literam S fero mentiet.

24uscuncrne mebelmåttigt långa meอ fort, thbligt affiltb, omyänbt ảggrunb ods fammantrọdt flubba.

Sonfrunoct ftort, näftan lifa breot fom thorax, förfebt meb en upy. fånenbe, belab, af långa yår bildab crifta emellan ägouten od unber antennerne. 
Palperme fammantrgefin, utfaienbe, bibergeranbe, flällige ods trelebabe:

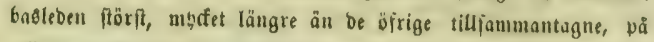
mibten utwibgab, mot lebfogningen fmanisisgom affmalnande, nã. flan răt, unoertill förfeob med långa, ulfperrabe bår, hreilfa näitan bölla be öfrige leberne; mederfta ods fifta leben ganfta [må, ล̊ggformiga, tillauman fnapt öfwerfliganbe baslebens trebje bel, ben meblerfta lifwäl något längre än ben fifta.

Sooneu natna.

Füterue finsemellan lifformiga; lioren unbertill glejare båriga; tibterne och) tarferne oruäpnabe; be förra utan tänder wib tarşleben. Tariens 1-4 leber uäftan lifftora, ben 5 te minft.

W3iugnrue belbräbdabe, lancettiormiga. Diff fältet litet, enapt inta. ganbe trebtebelen af twingen.

Merverue på bafwingarne 8: 1:fta från bajell, 2:bra, jum är twå. grenig, från ofre nervftammen, $3-6$ från nebre nervftanimen,

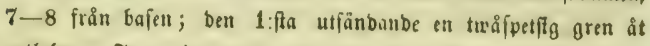
rotloben. Frammingarnes nerver 8 : ben 1:fta jrån bafen, 2-4 från nebre neruptumumen, $5:$ te från biffältets trernery, $6-7$ från sfre neruftammen, 8:De återigen från bofen; ben 7:be fent: grenig. Diffäliets twernerb på framuingarne lifnar ett lutinft $S$.

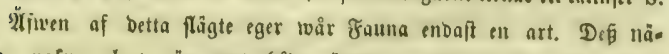
flan nafua larb är mot bäba änbarne affimalnanbe ody pupan

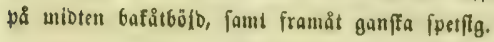

\section{Alunänna Nvitvingen (Leucophasia sinapis Lin.).}

Alis albis, anticis supra apico infuscato; posticis infra flavidis fasciis duabus transversis obsolotis infuscatis. L. al. oxp. $40-45$ millim.

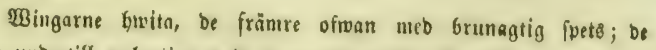
bafre unbertill gulagtiga meb twenne otboliga, Grunagtiga twerbant. R. e. wingfp. $1 \frac{5}{8}-1 \frac{13 / 16}{16}$ t.

Syn. Pap. sinapis lin. Is. Sv. 1038. Nabr. E. S. III. 1. 187. 577. Hüln. Pap. t. 82. 1. 410. M11. Text. 64. 9. Pontia Behs. Schim. I. 2. 169. IV. 31. Pieris 
God. of Dup. Lepid. I. Iol. 3. bis. pl. 2, tert. f. 4. Gunoris Dalm. Yap. si. 88. 7. Kett. Ins. Lapp. 907. 1. Leucophasia II-S. Text. I. 98.

Denua art ftunes i ffogar ber ud lytwar allmän i slal od)

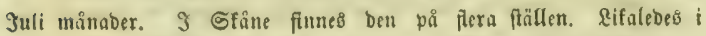

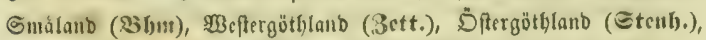
(3)ottland ods Diland (3ctt.), Hipplanb, 2Beftmanland ord Dalarne

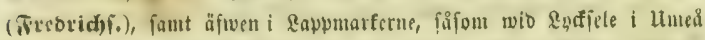

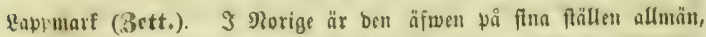
läjom mid Glyriftania, 5osferg, (5ger orh ufi Singerige (Gicbfe).

Rrtbefr. 2utennerne ofwan fwarta med Gwita ringar, unoertill hwita. Slubban inart, unbertil i inore iälften lywit. SBalperne

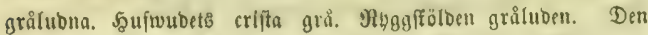
fmala abbonen ojwan glefare, unbertill tïtare brvitpubrab. Bröftet lywitagtigt, lubet. \$ôtterne lytuitagtiga.

Sanue od) Goua. SBingarne ofwan buvita, be frămre wib bafen od) utåt framfanten fint fwartpubrabe, meb en flörrecller minore, Grunagtig, gywituubrab, runbab fin̆ i fpetfen. De bafre oftwan något flammiga, till fölle af unbre ftonns färgtefuning.

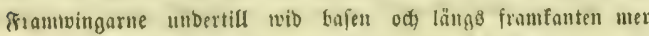
eller ninbre frunpubrabe, $i$ ipetfen gula. Bafvingatre gula meb Grunagtiga atomex, Grvilfa wib roten äro täta ords $i$ biffen hopa fitg till twenne otybliga twerbano. Franfarne Gwita.

Larven, fom fall lefwa pâ lotus ody latliyrus, är grön med en bögsgul linia lămgs fuarbera ftban. Wupan gar en röbbrun fooftrimma, bwila luftbål ods brunftrimmiga wing= flibor (Ddj).).

\section{Slïgtet : Muthocharis. Boisp.}

(Pieris Latr. Zettr. Pontia liabl. Ochs. Gunoris Dalm.)

Antenua mediocress distinclo clavata. Clava oblongo-ovala, compressa. 
Capat magnum, thoraci latidudine fare mquale, eriela ox pilis longis, densis divaricatisquo, orecta, inter oculos et sub antennis instructum.

Palpl compressi, porrecti, squamati, inlra pilis longissimis divaricatis dense instructi, triarticulati. Articulus medius basali longior, articulationom vorsus sensim attonuatus; ultimus, secundum longitudinem suam totam conlormis, minimus, apico rolundato, in pilositate reliquorum lere occultus.

Deull nudi.

Pedes invicom conformes; fomora infra pilis longis hirsutissima; tibiæ tarsique, præsertim posticorum ut in gonere sequente, sic etiam articuli farsorum, primus horum tamen tribus tantum proximis conjunctis lonzูitudine cequalis.

Alæ rotundatz. Collula discoidalis dimidiam partem alæ uccupat.

Costa alarum posticarum ut in genere sequento; 1:ma tamen ramulum rectum, ad apicom angulatum ot rofloxum, in lobum basalom omiltens. co-te alarum anticarum 9: 1:ma - hasi, 2:da-4:ta o trunco communi inferiori, 5:ta o costa transversa cellulie, tili-8:va e trunco superiori, 9:na a basi egrodientes; 6:ta quadriramosa, Costa tranıversa cellule al. anticarum inter costam 4 el 5 valde arcuata, convexitatom erga cellulam vertens.

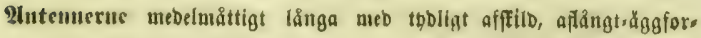
mig od) fammantrbatt flubba. .

Soufwubet ftort, näftan lifa brebt fom thotax; enellan ögonem od unber antennerne föríebt meb en uppfienbe crifa, bilbab af långa, tăta ợ utiperrabe Gadr.

Palperue fammantrhofta, utfáende, flälliga, unbertill förfebda meo

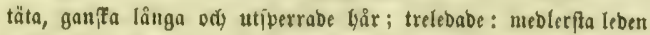
längre än bableden, utot lebfogningen jmåningom ajimtalnanbe; fifta leben längs bela fin längo lifformig, ganffa liten, meb run. bab ipetó, od náftan bold i be öfriges hårighes.

Ṡgouen nalna. 
Fotterne fincemellan lifformiga; liven unbertifl ganffa lubna meb

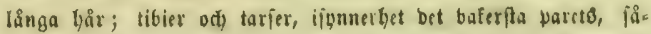
fom hos föllanbe flägte; få äfwen tarsleberne, men ben förfth of beşa är lifwäl blott lifa lång meb be 3:ne närmafte tilliam= mantagna.

Tzingarne rumbabe. Difffältet intager balfwa belen af wingens långb.

शerverue på bafneingarne făfom hoo föltande flägte; ben förfta fänber lifwảl àt rotloben en rät, $i$ ipetfen winflad och) utåto bölo gren. Framtwingarnes nerver 9: Den 1:fta från bafen, 2:bra-4:be från nebre'nervftammen, E:te frain biffältets tures= ucrb, 6:te-8:be från b̈fre netbftammen od) ben 9:be fro̊n bafen; ben 6:te fasgrenig. Diffältets suverners på framuvinganne emel. lan 4-5 twingnerwerne bågbỏib, wänbanbe ben fonvexa belen àt bif̣t fältet.

¿iffom af Gåba föregånbe flägten har wår frauna ef eller mer

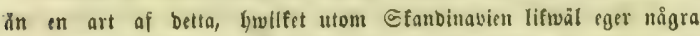
flera. Sarben lyar famula form fom föregående flägtes, ment pupan är något mera bafítbölb od mot båba änbar affpetfab.

Brisme-Auroran (Anthocharis cardamines Lin.).

Alis albis, anticis apice et macula sublunata media fuscis, maris modio aurantiaco-fuivis; posticis infra viridi marmoratis. L. al. exp, 40- $\$ 5$ millim.

WBingarme byrita, be främe 1 fpetien fruartoruna off 1 mibten en flär af famma färg; foo baunen i nibten orangegula; be bafre unbertill marmorerabe mcb grönt. \&. e. เvinglp. $1^{5} / 8-1 \frac{13}{16}$ t.

Syn. Pap. cardamines Lin. F, S. 1039. Kabr. E. S. III. 1. 193. 600. Ilïbn. P’ap. t. 84. f. 424. 423. t. 83. f. 419. 420. ot fig. 791. 792. Text, 65. 13. Poutia Dchs. Sclam, I. 2. 165. IV. 31. 155. 488. Treitsch. X. 1. 90. Pieris God. ot Itup. Lopid. I. Col. 3. bis pl. 2. f. 2. pl. 2 quart, 1. 1. Zett. Ins. Lapp. 907. 5. Ganoris Dalm. Pap. Sv. 86. 6. Inthorharis II-S. Text. J. 98. Denna uafra fiäril förefommer pả ångur allmänt $i$ fobra od 


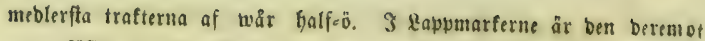
mera fällitynt. Den fibger blott en gång om året, $i$ Mal od börfan

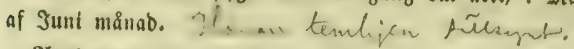

2rtbeffr. S2(ntemerne fwarta, lytwit eller gulpubrabs. STubban mo̊rface, unbertifl od $i$ ipetien gulagtig. Balperne unbertill

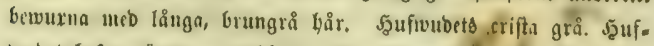

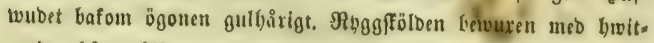

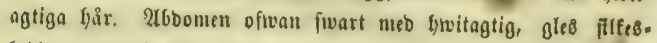
lubd; unbertifl brvit med lywit lubb. Fötterne funlta med gula agtiga tibier obt tarier.

Sonur. Mingarne Guita, ofwan wib bafen od i be fram. reb framfant fwartagtigit. Frnmuingarne ofwan i fpetfen froarts

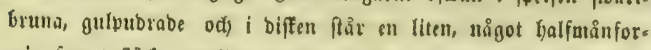
mifs (mart paaff; emellan benna od) ben juartagriga wingfpetfen äro wingarne från framfanten till bafre börnet orangegula, Grvilfet åfwen är förhållanbet på unbre fiban, ber lifwäl wing: ipetfen är lywitagtig med gröna llïfar. Den fwartn biffiäåen

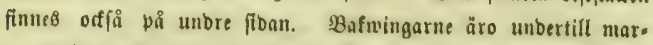
morerabe med gulagtigt gröna fläfar eller orebigt fammanlö. löpanbe Gano, lyvilfa på ôfre floan funas orbbligt igenom. Frane jarne Groita, wå framwingarne brunfläđiga.

souna lifnar Gannen, men fatnar den gula flăợen på framuingarne od) bar 1 besafas fpets mera frunt, faust 1 biffen ftörre täct.

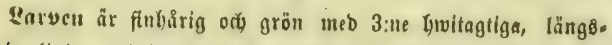
gående linier, af hrvilfa ben medlerfta år otopblig. Den leiwer enछిig på Cardamine pratense, l'urrilis glabra odj Brassica campostris. Wupau är grỏn eller foōtanbe i gult, flät, meo

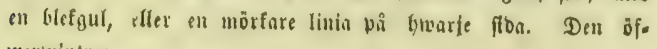
merwintrar. 
Slägtet: Pieris. Scrrank. LaAtr. H-S.

(Pontia Fabr, Ocas. Ganoris Dals. Anthocharis H-S. ex parte).

Autenut Iongiores, distincte clavatse. Clava obovata, brevis, dotorminata, compressa.

Caput uagnum, thoraci latitudime fere æquale, crista orecta, fere indivisa, ox pilis longis divaricatis ut in prioribus instructum,

Palpi *) compressı, porrocti, divergontos, squamati, infra pilis longis divaricatis instructi, triarticulati. Articulus basalis of modius aut inflatis ant normales; ultimus in pilositate reliquorum non occultus, longior.

Deuli mudi.

Pedes inter se conformes; fennora infia hirsula; libiæe tarsique dentibus brevibus instructi; tibire quatuor postica ad insurtionem tarsorum calcaribus brovibus munite. Articulus Lirsorum primu l'eliquis conjuncti. lero tequalis, secundus terlio, hic tamen quarlo longior, yuintus tertio longitudine wequalis.

Alse integree. Collula alarum d:scoidalis magna, dimidiam partem aloe aut occupans aut superans.

Costa alarum poslicarum 9: 1- 4 trunco communi superiori, 5-7 o trunco inforiori, 8-9 o hasi egredientes; prima ramulum simplicom, arcualum, acuminatum in lobum basalem

-) 'Tabuls soatomica forman palporum anticulorum exhibens:

A. Palporum articulus basalis et medius normales. Omnes inter - $\theta$ longitudine fere aquales: $P$. daplidice.

B. Palporum articulus basalis et medius tumidi, quasi inflati.

1. Art. medius ultimn fere longior; hic gracilis, apicem verous scneitn altcnualus: $\boldsymbol{P}$, napi et rape.

2. Art. medius ultimo fero squalis; hic ut in subdivisione priore: $\boldsymbol{P}$. brassica. 
emiftens; 2:da simplex. Costæ anticarum o: 1 e เหa $\mathrm{i}$, 2 -4 o trunco communi inforiori, 5-8 o trunco communi superiori et 9 e basi egredientes; $6: 1 a$ biramosa. Costa transversa alarum anticarum inter costam 4 ot 5 recta, obliqua.

Hutenucrne temligen långa, med sgolig flubba. Dennta omwånot äggformig, fort, befämo ody fammantrọdt.

Souftunbet flort, näftant lifa Grebs med thorax, forjedt meb ent nüs ftan obelab, af utiperrabe hår bilbab crifta, belägen på famma fỉalle fom ho fôregåenbe flägten.

अalperne fammantrb̧⿰ta, utåt rigtade, bibergeranbe, fiălliga, unber. till förfebda meb utiperrabe bår, rrelebabe: Gasleben och ben meblerffa antingen uppblåfa eller normala; ben fifta bolb $t$ be อ̈friges hårigbet od4 temligen lång.

SBones nafna.

Fötterue finsemellan lifiormiga; låren unbertill (ubna; tibier v(d) tarfer förfeoda med forta tänder; be fibru baferfa tibierne wib tarfens lebfogning betwäpnabe meb twenne fortare fporrar. Förfa tare̊leben näftan lifa lång med alla be föllande tillfammantagna; ben 2:bra längre än ben 3:bie; Denne längre än ben 4:be; ben 5:te lifa läng meb ben 3:ble.

R3iugaruc belbräbbabe. Difffältet antigen ułptaganbe balfwa wink gens läng eller öfiverftigande benjamma.

Rerucrue på bafwingarne $9: 1-4$ frăn öfre neruftamumen, $5-7$ från ben ncore, 8-9 från bafen; Den 1:fta utjänbande åt rots loben en enfel, bölb ody fpetfig gren; ben 2:bra enfel. Frants soingarnes nerber 9: 1 från bafen, 2-4 från nebre nerbftam= men, 5-8 från ben bีfre ดđุ 9 från bafen ; ben 6:te twågrenig. Twernerben på framtwingarne emellan $4-5$ mingmerwerne fueb od) rät.

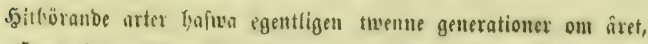
eburu flera af Dem finga bela Dent warmare tiben. Deras larber äro

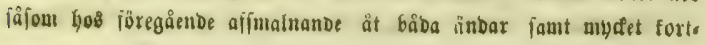




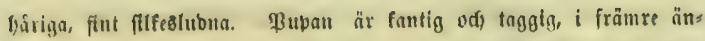
Doll entaggig. Gåmon larver göra cll bel arter temiligen ftor ffiba ไå föfsิเvexter.

\section{A. Palpernes leder năstan lika lingra; den medlersta och basleden normala.}

\section{Koifverfaddraren (Pioris daplidico lin.).}

Alis albis, anticis supra apicibus nigro maculatis maculaque ejusdom coloris in medio; potiseis infra griseo-virescentibus maculis difformibus albis. L. al. exp, $45-50$ millim.

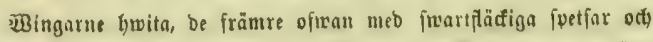
en flä af af fumma färg $i$ biffen; be fafre unbertill gríagtigt gröna meb olifformiga lyvita lärar. \&. e. wingip. $1^{13 / 16}-2$ t.

Sỵn. Pap. daplidice Lin. S. N. I. 2. 760. 81. Nabr. E, S. III. 1. 191. 595. Ilïbu. Pap. t. 82. f. 41\%. 445. et f. 777. 778. Text. 63. 6. Pontia 0ehs. Sclım. I. 2. 156. IV. 31. Treitsch. ג. 1. 89. Pieris God. ot llup. Lepid. I. fol. 3 bis pl. 2. secund. T, 3 pl. 2 quart. f. 2. Gauoris Dalm. Pip. Sv. 88. 5. Anthocharis II-S. Toxt. I. 98.

Flärilen, fom förft fåom fwenff upptäftes of wiar utmärfte Entomolog, ßrof. 3etterfteot, förefommer egentligen : twenne generationer om året, men woirgenerationen, fom finger $i$ Mlaj, år ganffa

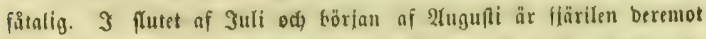

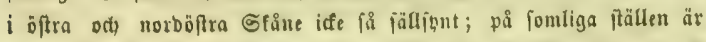

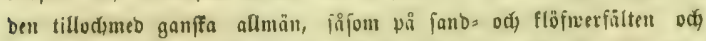

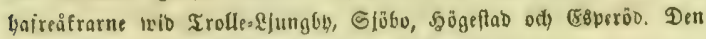
frall äfwen mara funnen $i$ Doftergötflanb (Dalm.).

Urtbefrr. 2(ntennerne byrrigrå mé mörfare ringar. Balperne

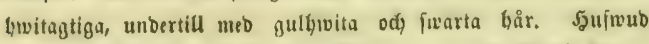
od roggföld ofwan gri̊lubna. 2(6bomen ofwan od) pi fiborme

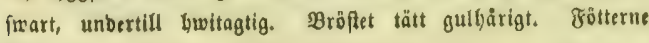
huitgula.

Saaurc. SBingarne ofwan fwita, wib bajen fwartagtiga. De främre bafwa $i$ fpetjarne flera fammanilytanbe, fwartbruna 


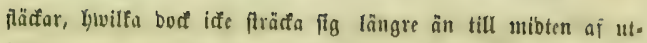

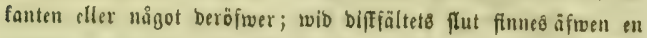

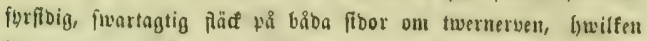
ismes fåfom ett fint, buitt fref, belanbe flüden. Bgafivingarue i utfanten omfring nerverue obetybligt fwartfuggabe; unbre

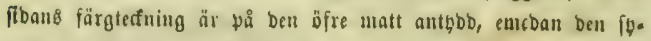
nes igenom wingen. Lnbertill hafwa framuvingarne $i$ fpetfen,

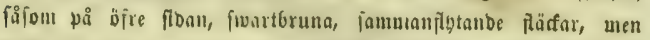

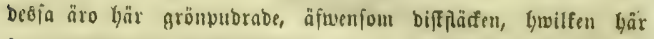
fanmanbänger med frautanten; nåra bafre börnet finneß äfwen oftu en grönpubrab mörf pärf, eller ofe blott antybning bertill. Bafwingarne äro ๖å unbre fiban gråagtigt gröna med olifior. uriga Gwita flätar, af rymilen twenne finnas $i$ framfanten, en $i$ biffältet, anbra bilda elt olifiormigt och aforutet tmerband Bufwer biften ody anbra, fom merenbelo ảro aplänga, uppiziga från flelîwa utfanten.

Souran lifnar batnen, men bar framuingarne mera ajruns

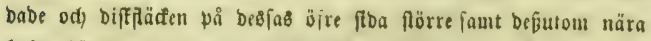
bafre bỏrnet en annan bylif flü. Bafwingarme äro ofwan $i$ biffen mörfare, emeonan unbre ftbans färgteđ̆ning funes tholigare igenom ods befutom anthbes af fwartagtiga ffuggningar; längs utfanten fimes ett twerband af fiörre fwarta, balfmånformiga fảctar, fom fammanbänga med andra. runbabe i fiel fwa uttanten.

Enven, fom lełwer på pleta återwexter, jåjom flöjimer, Jurritis glabra, Sisgmbrium sophia ody Brassica, ât mötfblì, guls ftrimmig och jwart punfterno, me liusgrönt, gulfäafigt od jwartpunfteradt qufwub. \$upan är grå, grön eller brun ods lifnar ben till följande art. (Odj). .

\section{B. Palperues Ieder sinsemellam olika långa; den med- lersta och basleden svilda, liksom uppolista.}

2. Mapsatfladdraren (Pioris napi Lin.).

Alis albis, anticis apice inluscatis; omnibus infra costis dilatatis fusco-virescentibus. L. al. oxp. 50-55 miltim. 
TBingarne buwita, be fräurre $\mathrm{I}$ ipetîen frunagtiga; alla umbertill

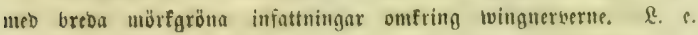
wingip. $2-2 \frac{1}{4}$ t.

Syn. Pap. napi Lin. F. Si 1037. Nalor. E. S. Ill. 1. 187. 576. llïbn. Рap. 1. 81. โ. 406. 407. ''est. 62. 4. Pontia 0chs. schm. I. 2. 149. IV. 31 ef i53. Treitseh. 1. 1. 88. Pieris God. of Hup. Lepid. I. fol. 3 his pl. 2. quarl. lig. 3. pl, 2 tort. f. 3. Lett. Ins, Lapp. 907. 4. var. a. II-S. Toxt. I. 96. (Aanoris Malm. Pap. Sv. 87. h. Rapsat risslinn Dahllb. Sh Ins. Skilda och Nytta p. 158. 111.

I'nr. b. Alis ant alhis aut sordide lavicantibus, anlicis supra maculis duabus luseis; postici- intra flavis; omnibus utringue costis dilatate infuscatis.

WSingarne antingen brvita eller fmutifgt gula; De främre piwant urb twenne bruna flärfar $i$ biffen; be bafre unbertill gula; alla på bảba flbor med breb, brun infattning omt: fring wingnerberne.

Syn. Pontia Bryonia (Napi var.) Ocls. I. c. obs, p. 151. 152. Gan. napi var. Dalm. I. c. Pont, napi var. b. Zett. I. c. Pieris Bryonice God. et Dup. l.eprid. Jl. 39. pl. 5. f. 1. Pieris napi var. II-S. 'Toxt. I. 97. IIülı. Pap. t. 81. f. 407.

Denna art förefommer allmänt öjwer allt inom Stwerige od Notige. Barieteten, fom af flera Förịattare blifwit anjeob för egen nrt, är lifwäl egendomlig för be norbligafte lanbffaperne, ber ben ef

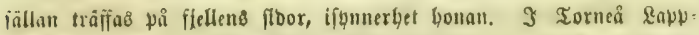
marf fann \$rof. Bettcriftot båba varieteterne. 2trtens fühgtib in.

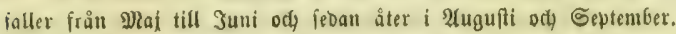

Irtbeff:. Antennerne fwarta, Gwitringlabe. Siubban pwart, i

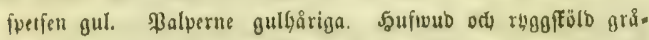
flone. 26bomen ofwan fiwart, unbertill lywitgul. Brofter Gwitgult, lubet. Fötterne Gะvitgula.

Şaute. WBingarne ojwan lywita meb fmala, Erunagtiga nerser. Eranıwingarne wib bajen ods i framfantena inbre bälft fwartagtiga uted en fwartbrun flä i $i$ wingfpetien, upplöfande fig 
I uffanten till brun fruggning ontering nerberne; I biffen alt. tingen oflärfabe eller meb en brunagtig, merenbels otholig pärt. Bafningarne wio bajen frartagtiga od i utfanten omfring ners verne fint iwartbrunt faggabe, faut oftaft meb elt invartbrun färf i framfantens mibr. WBingarne unbertill: be fräure lquita meb gulagtiga mingfpetfar, be bafte gula; alla meb gröu, utbrebe infattuing omfring nerserne, iisnnerlet be bafre. Jumnarne buvita.

Syonan lifnar bannen, uten bar mexn afrunbabe twingar,

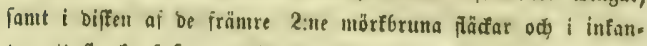
ten ett frect af famıma fărg.

23ar. b. frilfer ftg irån föregående entoaft beri, att både

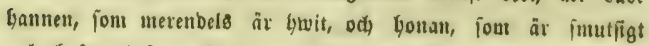
gul, bafwa $i$ frammingarnes bift twenne mörforuma fläđar, faut att alla nerwerne pä öfre fiban bajwa breb brun infattning, iå att wingarne få ett fmutfigt brunt utjeende. Şonan är mörfaft.

Earven ä finfirig, blåagtigt grön meb ets fint, otģbligt,

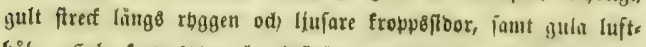

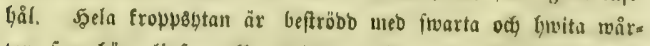
tor, font bära liufare eller mỏrfare bår. Den lefiver på Så̊le werter, Reseda od Turritis. Wupan är guigrön meo iunta punfter od) gula od jwartifăfiga fanter.

Rarven till benna ocis follanbe arter förorjafa ef ofetyblig ffaba på fålwexter, Gtwaxföre man fỏreflagit affilliga mebel till förefommanbe berai. Des̄ja upptagas̊ utförligare uti åtfilliga

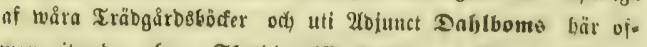
wan citerabe arbete: Sfanbinaviffa 3njefternes ఇotta od) Sfaba, bwarföre iag Glott will bämvifia till besja. 2fforb̧ter man od! fôrftör de Glab på tålwexterme, ber bonorne lagt fina fmå, hos be fiefta arterne gulagtiga od) elliptiffa àgg, förefommes berigenom en betbolig bel af ben befarabe faban.

\section{Rofvefladdrareu (Pieris rapæ Lin.).}

Alis albis; anticis supra apicibus infuscatis, albido-punctu- 
latis; posticis infra flavidis nigro punctulatis. L. al. exp. 45-50 millim.

W3ingarne hyvita; be fräure ofwan i fpetjen Grunagtiga, Gwit= vubrabe; be Gafre unbertill gula med fina fwarta puntser. \&. e.

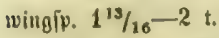

Syn. Pap. rapa Lin. F. Sv. 1036. Mabr, E. S. III. 1. 186, 575. Ilü̉bn. Pap. t. 80 f. 404.405 .7 ext. 62. 3. Pontia Ochs. Sclim. I. \&. 146. IV. 30. Gamoris Dalm. Pap. Sv. 87. 3. Pieris food. of Dup. Lepid. I, fol. 3 bis pl. 2. tort. I. 2. Zett. Ins. Lapp. 906. 3. H-S. T'ext. 1. 96. Rofvevissling Dahlb. Sl. Ins. Skada och Nytia p. 158. 110.

$\Im$ föbra od mublerfa Smerige ody Rorige ăr benna art lifa allmän foum forregående od Amger wib famma tio. I Rnpwmarferme är ben beremot fälignt, men buru bögt ben egentligen går mot norben ăr iđe utrönt.

2rtbefer. 2ntennerne lywitagtiga. Slubban brunagtig, i fpetfen od, umbertill Gwitgul. ßalperne lywitgula, lubna. Şufwub, rtgegföld, abbomen, bröft od fötter fâfom hos föregåenbe.

Sanure. 2Bingarne ofwan fyita; De frömre wib bafen, längs framfanten ody $i$ twisgipetfen Grunagtiga, Gwitpubrabe; $i$ biften finne flunbou en flä af a famma färg; be bafre bafwa

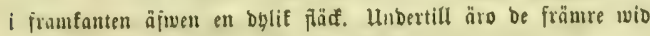

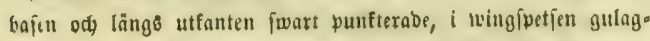
tiga ody bafiva $i$ diffen en brun häa. De bafre äro yå famma fiba gula med fina fwarta punfter, fom äro tätaft svid bafen.

Syounu lifnar bannen, men Ģar $i$ bifen \å framulugarnes

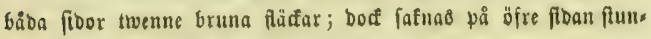
bon bell ena.

Sarven är finljårig, blångtigt grön meb tyblig, gul rดge* linia ody gula fläđar på floorne. Den lejwer på famma werter fom föregående och deltager meb honom i ben föröbelfe, fom

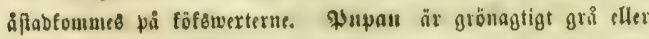
brunagtig med pwajwelgula linier. 
4. Kâlnaddraren (Pieris brassicæ Lin.).

Alis albis; anticis supra apicibus lato nigris; posticis infra flavidis nigro punctulatis, L. al. exp. 65-70 millim.

WBingarne Gwita; be främre ofwan $i$ fpetfen breot fwarta; be bafre unbertill gulagtigu, iwartpunfterabe. 2. c. wingip. $211 / 10$ $-2^{13 / 16}$ t.

Syn. Pap. brassicce Lin. F. Sv, 1035, Pabr. E. S. III. 1. 186. 574. Ilibun. Pap. t. 80. T. 401-403. Text. 62. 2. Poutia Ochs. Schm. 1. 2. 14k. et 2\%0. IV. 30. Ganoris Daln. Pap. Sv. 87. 2. Pieris Godl. ot Dup. Lepid. I. Fol. 3 bis pl. 2. tert. I. 1. Zett. Ins. Lapp. 906. 2. II-S. Text. I. 96. Kalvissling Mahlib. Sk. Ins. Skada och Nytla p. 155. 109.

Denna art äs $i$ fobra od mederfta belarme af Stwerige ody

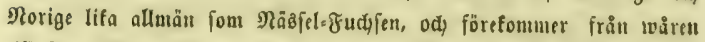
till fena böften. I egentliga Rappmarferne lär ben iq̛e finnus.

2Ittbeffr. 2(ntennerne frarta med Givita ringar. De ofrija froppẻbelarne âro till färgen lifa föregåendes?

Sanume. WBingarne bytrita; be fräure frăn bafen utmed framianten brunagtigt grå neอ en breo fwart, föga huxits

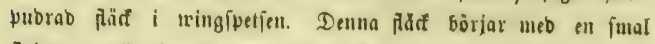
frrinıma wio framfantens frungrå färg ở flutar $i$ utuans ten, no̊got framou wingens bafre börn. De bafte bafwa on fiwart fä̆ $i$ framfantens mibt. Itubertifl äro framuringarne $i$ fietien gula ber be ofwantill äro jiwarta, ody hafwa $i$ biffen twenne rumba, fwarta fläfar. De bafre äro på famma fida. gula, tätt befröboba med jiwarta atomer.

soutan lifuar lyanmen, men bar ofman i framwingarnes bift menne runba, fwatta flăfar od) intill bezfa ett jwart firect, fom går fruebt frâul inbre fanten.

Enrven är funtyårig, jwartpuntterab od Glån̆rơn meb en

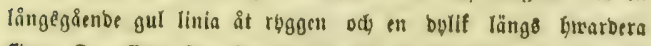

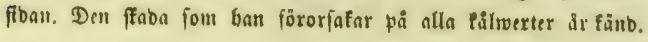




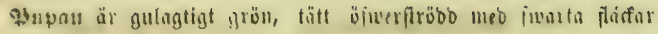
o(t) punfter.

Rüranbe mebel mot ben ffaba laruen åfrabfommel fe ofmur anförba arbete of 2(D). Snf)lbom pag. 156 ody 157, biraref? fábana uyprätnas mot alla fålmaftar.

\section{Sliagtet: Aporiala. Ilüв.}

(Pieris Latr, Zert. H-S. Pontia Fadn. Ochs, Ganoris Daly.)

Antenna longiores, extrorsum sensim crassiores, in clavam elongalam gracilem, haud delerminatam subeuntes.

Caput latifudine thoracis multo minas, fronte villoso.

Pulpl compressi, porrecli, valde divergentes, squamati, infra pilis longis divaricatis instructi, triarticulati. Articulus basa * lis reliquis conjunclis longitudine exqualis; medius ultimo nonilial longior, hic nudus, distinctus, apicem versus sensim attenuatus; basalis et modius normales non infati.

Acull nudi.

Pedes inter so conformos; tomora infia lirsuta; tibiæ tarsiquo dentibus brovibus serrati; tibia qualuor postice ad invertionem tarsorum calcaribu: hrovibus munilie. Articuli farsorum $\mathrm{ut}$ in genere præcodente.

Ale inlegra minus squamalie, ad margines prosertim anticarum plus minus denudatic. Collula discoidalis alarum magna, in anticis hatud, sod in posticis oxacte dimidiam partem alie

- superans.

Costae ala1 um posticarum 9 ut in genero priccedente, sed ramulus costre prima rectus, el inter costam 7 et 8 ptica, costam mentiens, in parto inforiore alarum adest. Costæ anticaram 9: 1 o basi, 2-4 o trunco communi inferiori, 5 o cos 1 a transversa celbul 6 - 8 o trunco superiori ot 9 ilorum a hasi egredientes; 6:ta triramosa. Cosla Bransvorsa cellulie modice oblizuno archala. 


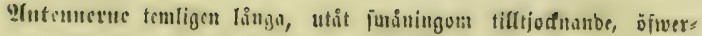
gåenbe till en ailång, jmal odj jöga affetilo flubba.

Suffubut miscet minbre än mellanfroppen $i$ brebb, meb luben biesja.

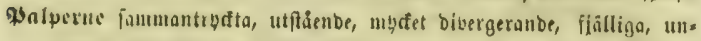
Dertill föricbo meb långa, bivergeranbe gair, trelcbabe: bastsoen lifa ling meb be örtige tillfammantagna; ben meblerfia mågot

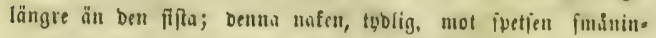
gom aifualnanbe; Gasleben od) ben mederfta normala, ire upj. btåta.

Souncu nafna.

Fotterue finemellan lifformiga; låren unbertill lubna; tibierme od tarferme tagsiga of foria tänber; tibierne wid turjens lebjugning wäpnabe ned forta iporrar. Tarjens leber fäjom hos föregående fägte.

23ingatue qel6räbdabe, glejare fjällige, mot fanterne mer ellet min *

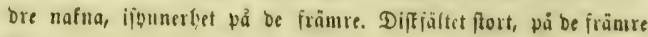
fuapt, men på be bafre tybligt ojwerfiganbe wingens fualfnes lăngo.

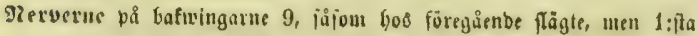
nervens gren rät ody emellan 7 -o wingnesberne finnes unber. till ett wert, fou är juarlift en uerv. Erammingarnes nertuer 9: 1:\{ta från bafen, 2-4 från nebre nervftummen, 5 från biffältets twernerv, 6-8 irån Bire nerbitammen ory 9:0e àterigen från lajen; ben 6:te tregrenig. Diffältets tweınerb olețibligt fnebt bojib.

Rurben till betta fägte år temligen lángluben mob längre bậ

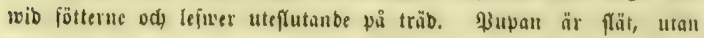
taggar, men meb en runbab upvimällnitg pí qujubregiontu.

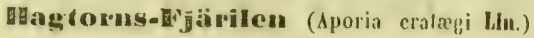

Mlis allis costis nigris. I. al. oxp. $65-70$ millim.

Bingarne fnita med fwatka nerber. \&. e. Wingip. $211 / 10-$ $213 / 16$ t.

Syn. Pap. cratagi Lin. F. Sv. 1034. Vabr. E. S. III. 1. 182. 563. Mlïbu. Pap. ใ. 79. f. 399. 400. Toxı. 61.1. 
Pontia Ochs. Schm. I. 2. 1'2. IV. 30. Gauoris Balm. P'ap. Sv. 86. 1. Pieris God, ot llup. Lepid. 1. fol. 3. bis pl. 2. f. 3. Zett. Ins. Lapp. 906. 1. II-S. Toxt. I. 95. Hagtornvissting Dahllb. Sti. Ins. Skada och Nytta p. 154. 108.

Desna art träffå $i$ päbra od) meblerfta probinjerne of Swerige

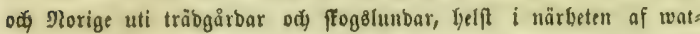
ten, ody är ber temligen alumän. I egentliga eappmarferme är lyan

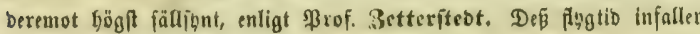

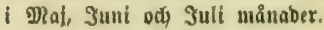

2rtbeff. 2(ntennerme iwarta. Rlubban $i$ fpeten Givitgul. \$al:

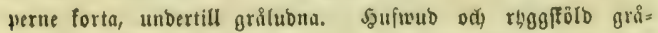
lubna. 2Gbomen ofwan jwart, unbertill Invitagtig. SBöftet gwitgult, lubet. Fötterne fwarta med lyuitgra långftred.

Saaute ody boun. Wingarne enfärgabt lywita meb fwarta nerber; be bafre unbertif ftatanbe 1 gult.

Sarven ẫ gråluben, mé fwarta od̆ orangegula ftrimmor längs ruggen od) affeblå flbor, fywarbeta meb en rab fwarta punfter. Den lefwer på bagtorn, plommonträb od flera anbra

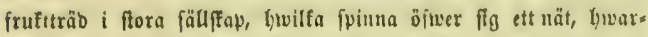
unber be lywila ody föfa fepbo wib annalfanbe otwäber: Rarven

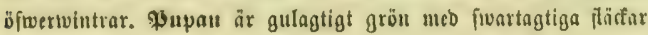
Dळi puntter.

Earven fablig, emeban den om tvåren uppäter blab uch Glomitnopyarme på fruftträben.

\section{Trib. Parnassii (mabr.).}

\section{(Parnassides Dur.)}

Pedes 6 omnes gressorii, intor so conformes.

Cellula diecoidatis alarum posticarum cosia framsersa coloris eequali cliusa; anticarum conla in medio abrupta subchasa. 
Margo anticus alarum anticarum costis trilus simplicibus, nempo costis alie 8-10, oxtendilur.

fosta alirum posticarum 8: 1 o trunco communi cum q:da et 3:tia ratice simplico ogrediens ot ramulum rectum simplicom in lobum basalem eniltens; 8 iterum e basi egrodiens. Costæ anticarum 10 ad basin invicom subequales, 7 triramosa, 1 ot 2 a basi egredientos.

Ala postica rolundate, pro abdomine libero in margine interiori excisre.

Larra pilosa, cylindrica, capite tentaculato.

Pupa lævis, mutica, subfolliculata.

Tïtterue: alla jer gångfütter, finßenteflan lifformiga.

Diffeältet pi̊ bafwingarne flutet genom en twernerv, fom är lif uvingnerverne. Diffïltet pô framuingarne balffutet genom cn Џå mibten -afbruten twernerb.

Fromfauten af frantwingarne bălles utipänd genom trenne entla nerver, neml. genoul 8-10 ivingnerberme.

Sicrverue på bafwingarme 8: Den 1:fta utgåenbe meb en entel rot

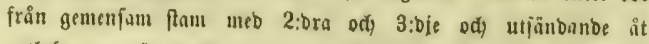
rotloben en rät, enfel gren; ben 8:be återigen från bajen. Fram. wingarnes nerver 10, wib bafen lifformiga; ben 7:be tregrenig; ben 1:fta ody 2:bra utgåenbe från bafen.

Bafuingarue runbabe, $i$ inore fanten utfurna för ben fria ab. boment.

Sarve'u luben, çlinorift, meb eft utfräđfourt fpröt i bufwubet.

अ upan flät, utan taggar, liggnnbe $i$ en näfan fofonglif bolia.

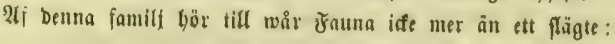

\section{Slägtet : Doritis. FABn. et Aucr.}

(Parnassius Latr.)

Antenne brevos, clavale. Clava subovata, recta, incrassate, apico ixueronato.

Palpi capilo Iongiores, subadscendentes, prominuli, pilis longis fimbritt, triarticulati. Irticulus basalis arcuatus, omnium 
latissimus; modius of ultimus recli, proportio eorum tamon pro specie variat. *).

Deuli mudi.

Peles conformos; fomora infra pilosa; tibire tarsique dentibus minutis serrati; libie quatuor postice ad insertionem tarsorum calcaribus duobus longis munite; articulus primus larsorum duobus proximic longitudine aequalis; 2, 3 ot 4 inler so longitudino lero requales; quintus tauen tortio yuartoque conjunctis requalis.

Ala integra, parcius squamalie, subnudx; priesertim infra ad marpinos denudatæ.

Costae alarum posticarum 8: 1- 3 e trunco communi superiori, 4-7 o trunco inforiori, 8 o basi ogredientos; 1:ma radices simplex ramulum rochum, oxtrorsum allenuatum ot simplicem in lobum basalem imittit. Costa anticarum 10: 1:ma, minima, et $2: d a$ o basi, $3 \longrightarrow 6$ o trunco communi inforiori, 7-9 o trunco superiori of 40 o basi egredientes; 7:ma triramosa. Cosla traniversa alarum anticarum in medio inter costam 6 et 7 abupta. tantum tuniditate connexa. Antennerue forta, flubbformiga. slubban något äggiormig, rät, tilltiodnab, uteb tagg i ipetjen.

وalperne längre ån bufuubet, Hågot uppainfigande, framftiesioe, franfabe med långa bair, trelebabe: bagleden frött, brebare än

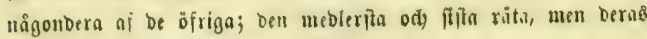
proportion varierar efter arten *).

Shonen nafna.

Tötterue lifformiga; låren unbertill lubna; tibier ofl) tarfer fågabe mebelit forta tảnber; be fura bafre tibierne wio leojogningen

*) Tabula analonica proportionem articulorum palporum exhibens:

1. Articalus basalis longissimus (unicuique reliquorum longior); medius ultimo longior, hic brevissimus onnium, conicus: mnemosyne.

2. Arliculus basalis medio, hic tamen ultimo ferc brevior; ultimus longissimus omnium, gracilis upicem rersus sensim altenuatus: apollo. 


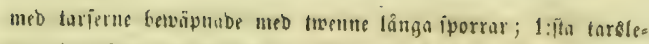
Den lifa ling me be tweme närmafie, 2, 3 vic) 4 finkemellan nåßan lifa långa, men ben 5:te lifa med ben 3:bje vdy 4:De tilljammantayura.

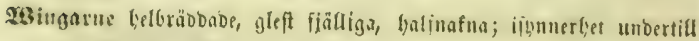
mot fanterne blottabe froin flall.

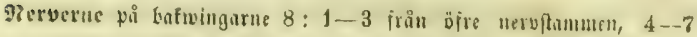
frioll bell neore, 8:be frîn bajen; ben 1:fta, foum bur entel rot, mä̈nbor till rotloben en rät, mot fietfen ajimalnande od enfel

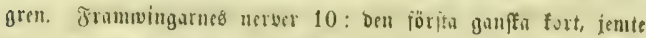

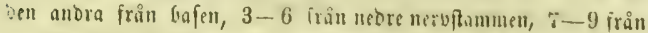
Den öfre, 10:De från bufen : ben T:De tregrenig. Frambyingarnes swiancry cmeflin 6 och 7 tringnerterne af́bruten

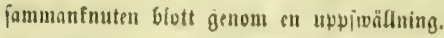

De wadra arter, fom böra till Detta flïgte, iubrefomma enbaft i bergiga fogetrafter od) bujwa blott en genetation om året. Deras

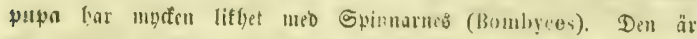

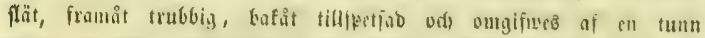

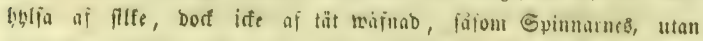

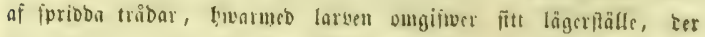

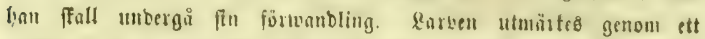
twioclabt fpröt, fom fans inbragas od utfiutas, famt är öner

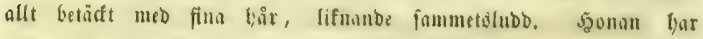

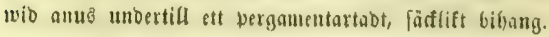

\section{Hvita Tannvingen (Doritis mnemosłno lin.).}

Alis albis costis nigris, anticis maculis duahu discoidalibus nigris. L. al. exp. 55-60 millim.

Bingarne Gwita ned fwarta nerber; De främre med twenne

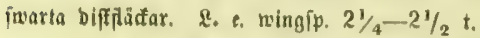

Syn. Pap. mnemosyne Lin. F. Sv. 1033. Vabr. E. S. III. 1. 182. 562. Hïhn. P'ap. 1. 79. 398. Text. 61. 7. Parnassius Hod. of Dup. Lepid. II. 21. pl. 2. f. 3. Moritis Ochs. Schm. I थ. 199. IV. 30. Treitseh. I. 1. 86. Balm. Pap. Sv, 86. 2. II--S. Toxt. I. 146. 


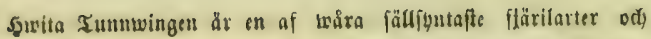

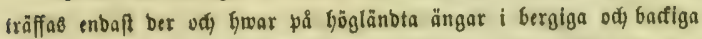
ftogstrafter af Swerigez fobligafte probinfer. Uti Efine är ben

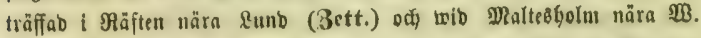

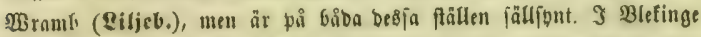

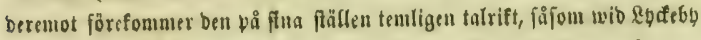

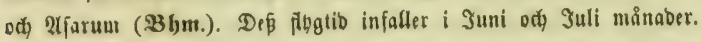

Irtbeffr. Intennerne od) Elubtan (warta. Sofwubet fwartbrunt,

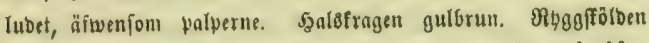
grångtigt luben. \&6bomen ofwan fwart meb gle gra grå bår; umbertill tätare gråluben, i fanterne med gulagtigt floft. ßer= gamentsbityanget hos yonan gullywitt. Bröftet grålubet. Föts terne fwarta, med glefl, gulagtigt ftoit ber od lywar.

5yanı. Wingarne ofwan bwita, $i$ fanterne Glottabe från

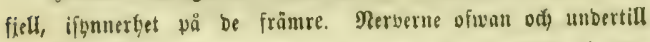
fwarta. De främre wingarne med twemne fwarta flärfar $i$ biff: fältet, Gyvilfa finnas på båba fibor. De bafre of man frůn lias fen längs inbre fanten fwarta; i framifantens mibt od wib bift.

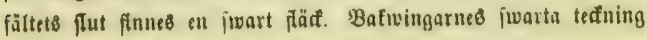
ignes äfmen på unbre fiban, clyuru matt ody ofboligt.

Sรоแกแ till färgen lif bamen.

Qarvell, fom är mig ofefant, ffall le fiwn på Corydalis cava.

2. Höanäckiga Tummvingen (Dorilis apollo Lín.)

Alis albis, anticis maculis 5 nigris; posticis ocellis supra 2, infra tribus, at ctiam maculis hasalibus, rubris. L. al. exp. 83 -90 millim.

Bingatme fyvita, be frămue med 5 furarta flădar; be Gafte of wan neb 2, unbertill med 3 röba occller od fläđar af famma färg wib bafen. \&. e. wingip. $3 \frac{3}{8}-3^{5} / 8$ t.

Syn. Pap. apollo Lin. F. Sv, 1032. V'abr. E. S. III. 1. \31. 560, llibn. Рар, 1. 79. 1. 396. 39\%, fig. 730 . 731. Text. 60. 6. Swartz Sv. Zool. II. 7. I. n. 41. Parnassius God. ot Dup. Lopid. II. 15. pl. 2. f. 1. Doritis Ochs. Schm, 1. 2. 132. IV. 30. ot 152. Treitsch. X. 1. 85. Dalm. Pap. Sv. 85, I. II-S. Toxt. I. 145. 
Bar. a. Ocollis magnis ruhris, alho pupillatis utringue (Dalm.) Ocellerue ftora, röba meb bwitn pupiller på bådo fibor af Iringarme.

Bar b. Ocollis supra rubris ahsuge pupilla alba, infra albo pupillatis (Malm, I. c.),

Deflerne ofienan röba utan hivit pupilf, unbertill med bufif pupill.

Bar. e. Alis maculis ocellarilus supna nigris, non nini obsolote rubro pupiltalis; infra yaoque sine pupilla alba (Dalm.). 78ingarme ofwan med fuarta, fuapt robpupillerabe oceller: unbertill äfwen utan bwita pupiller.

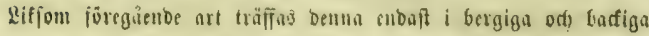
ffogetrafter, men förefommer lificis, sfumu temligen fälibut, $i$ alla

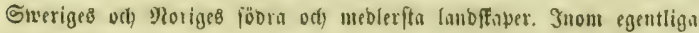

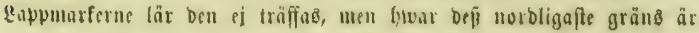
fänter man änแu si. 3 norra belen of WeftmanInto förefoumes

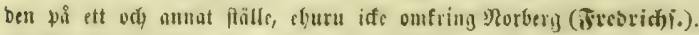

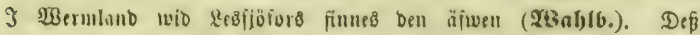

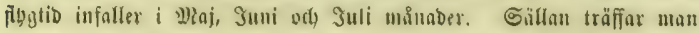
en od) anum lebuare. Borieteterne förefomma tillomman; ben fifta år bod wibn fällibntare ån be örrige.

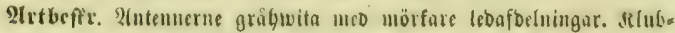

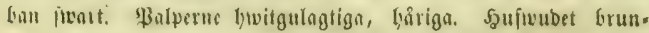

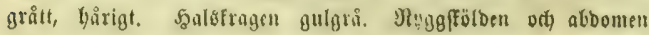
ofwan mro fyritgrå, lång lubb. 2(bbomen umbertill od) bröftet,

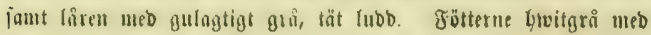
fwartu lebafochingar på tarferme.

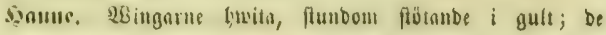
jränuc ojwan od) neban med fem fwatta fläfar $i$ biffen, of Gnilfa be farra äro belägne mib fromfouten ody ben femte, foum är runbad, nåra infanten. Unbertill är funbom en cller an. nan af bem rỏbpubrab. W3ib bajill od) länge framtanten finne?

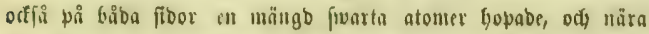
ben blottabe utfinten ell rnd fivarta, af atouter bifbabe fläfius, 
fout gâ frîn framfanten inåt wingen. Bgafuingurne äro vfuran från bafen längo inore fanten furartpubrabe och pai bíba floor förjebde meb tweune ftora, röba med jwart ring onigifna oceller, fom än bafwa luwita pupiller pâ bíba froorne, än bloll พ⿳亠 ben unbre, od fumbour fafsu fíbana, bi be äfwen aro föga röbs pubrabe. Den ena of beฮิfa oceller är belägen nära frumfanten, Den anbra $i$ diffen, bort närnare utfanten äแ bajen. 3 inbie fanten nära aualbörnet finnas ämentedes pí frída ftoor några jwarta tuerfaienbe fäctar, fom unbertill äro fundom röbpubrabe

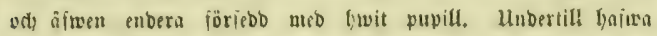

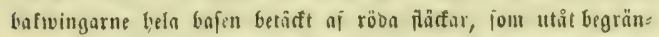

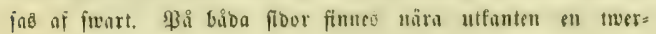
rab otboliga, genom fwarta alomer fillobe päufar.

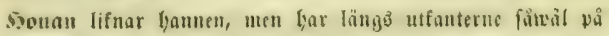
(rammingarne fom på bafmingarne mbligare flüarar ori) inbre fanten af bufwingarme ofwan meri uttrebt fitart. Det perga= mentartabe bifanget nib antเ är fwartagtigt.

Garven fammetsiurart med twenne rader orangeröba plüfar od) flexe frílblå wairtor, jamt lângre ftiffelbair bland ben fors tare lubden. Şufmubet är jirart med en brandgul flä $i$ naco

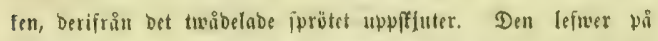
aiffilliga arter af Sedum, ifunnerlyet pia sodum tolophium. \$upan är $i$ börian blefgul, febau röborus vch) flutligen violett, öfiwerpubrab neb blågrått miöl.

\section{Trib. Buquiles. (H..)}

Pedes 6 omnes grossorii, inter so conformes.

Cellula discoidalis alarum posticarum costa transversa coteris sequali clausa, anticarum cosla transversa continua chasa. Margo anticus alarum anlicarum costis qualuor simplicilus, nempe costis alæ $9-12$, extonditur. 
Ceslap alarum posticaram 8 : 1:4la e trunco communi cum 2:ala et 3:tia radice duplice egrediens el ramulum bimueronatum in lobum basatem enittens; 8 iterun o basi egrodiens; anlicarum 12 ad basin invicem subequales, 8:va biramost, 1 et 2 e basi ogrudiontos.

Ala poutica rantatie, fro abdomine libero in margine interiori excis $x$.

Larra lesis, mula, fentaculats.

Pupa angutati, antico bimucronati, nuda, ano ef filo per midium transierso alligata.

Füterme: alla fex gingfơtuer, fimsemellon lifformiga.

Difrfältit bå bafningarne flutst genom in twernerb, fom är lif be örriga mingnelverne; Hî framuingurne lifalebes fluset genom ell onforuten thernerv.

Tramfanten pî framningarme balles utipino genom 4 enfla ners ber, nemiligen genom $9-12$ wingiterberne.

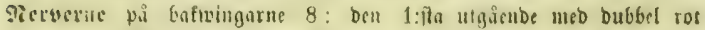

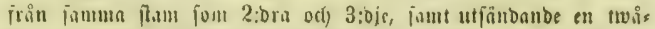
ivetiac gren at rotloben; bell 8:De äterigen frân bafen. Frnme wingarileg nerwer 12, who bajen finsemellan lifa; ben 8:De twa., grenig; ben 1 odl) 2 utgăende frîn bajen.

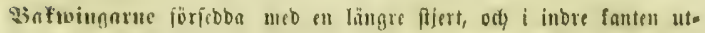
ffurna för ben fria aboomen.

Earven fät, nafen, med att utfträfthart fpröt i bufmber.

\$upan fantig; framtill twålaggig, nafen, faftyănganbe med anus oc mebelit en omfring mibten flugen tråo.

2ff benna, färbeles i ljeta zonen, pâ flügten oç arter rifa unber.

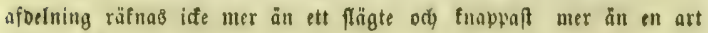
tiul wår Founa.

\section{Slägtet: IPapilio. (LAN.) Ocns. et Aocr.}

\section{(Amaryssus DaLx.)}

Autenmx longioros, vorsus apicom sensim crassiores. Clava oblonga, pyriformis, arcuata. 
Palpi Jarvissimi, adprossi, pilosi, triarticulati. Articulus basalis ot medius infer so fore aequalos, lati, lice timon rernus insertionem ultini nonihil altematus; articulus ultimus in pilositate procodentium plano oceultus, minimus, rudimentalis, lertiam partom articuli medii rix attingens, fero rofindaliss.

Deuli nudi.

Pedes inter se conlormes; lomora inlra pilosa; libic larsique fere ut in genero precolente sorrati ot armati; sic oliam proportio arliculorum larsorum, sod articulus quarfus minimus, ot 5 secundo fere requalis.

Ha postice dentata of caudate.

Costa alarum posticarum 8, omnino ut in genore precedonto, snd 1:ma rabicom duplicom, unum o basi ala, altorum o Irunco superiori eqredientom, labet of ranulum arcuatum bimucronatum in lobum basalom emiltit. Coste anticarum 12: 1:ma, hrovissima, ot 2:da 0 ba-j, 3-6, frunco communi inloriori, 7:ma o costa llansversa collula, 8-11 0 irunco communi superiori of 12 iterum o basi egrodiontes. Costa 2:da cum trunco inleriori costula transiorsa non procul a basi connectitur, ot costa 9:na ox codem loco collulæe discoidalis cum 8:ra, biramosa, egrodit. Costa transversa cellula discoidalis continua.

Plntemerne temligen långn, mot fpetien fmaningom tifltiofnanbe. Rlubban afång, päronformig, fröft.

Halperue mydet forta, tilltrbdta, gårign, trelebabe: Gasleben ody ben meolerfta findenuellan näptau lifn, breba; ben feonare lifuäl mot lebfogningen meb ben btterfla något affmalnanbe; Den fifta leben alldeles bold $i$ be föregåenoes lyarighet, ganfta liten, näftan rubi= mentảr, ợ runbab.

S̈gonet natna.

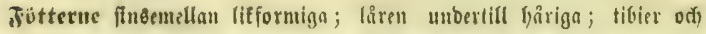

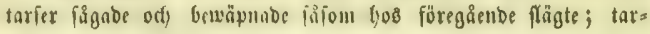


jens leber äfwen lita med föregåenbe fägtes, men ben 4:be mint o(1) ben 5:te näftan lifu lång meb ben 2:bra.

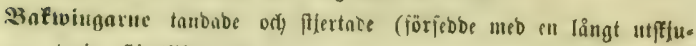
tande, fofertilit tanb).

Sicrocrue på bafwingurne 8, alloelfs fom goo föregående flägte, men bell 1:fta bat bubbil rot, en fommanbe frin baien odf ell annan ftล̊n ofre nervitammen, famt jänber ait rotloben en fröft odi) tmaipetifg gren. Framuingarmes nexber 12: ben 1:fla bögf? Fort lemte $b$ on 2:bra fommanbe från Gajen, 3-6 från nebre nervftaumen, ben 7:be från biffiältets tweruerv, 8-11 från öre nervftammen od ben 12:te aiter från Gajen; 2:bra wing. nerven förenas meb nebre nervftammen, ife laingt från bafen, jöruebelft en liten twernery, och) ben 9:be winguerwen utgå fråu jamma ftalle af biffiältet jom ben 8:be, hivilfen är twigrenig. Diffäitets tweenerw oaforuten.

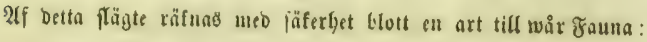

\section{ParasolI-Bjöimilen (Papilio machaon Lin.).}

Alis flavis costis nigris, maculis margineque oxteriori, flaro lunato, nitro-fuscis, posticis supra macula subocellari fulva ad angulum ani. I. al oxp. $80-90$ millim.

WBingarne gula mod iwarta nerver od fwartbruna fläctar ods utfant af famma färg; ben feonare me gula baljmanformiga fläfar; be batre ofwan med en robgul, näftan ocellormig täa $i$ analbörmet. 8. e. wingip. $3 \frac{3}{4}-3 \frac{5}{8}$ t.

Syn. Pap. machaon Lin. F. Sv. 1031. Nabr. E. S, III. 1. 30. 87. Ilübn. P'ap. I. 77. ศ. 390, 391. Text. 59. 2. Ochs. Schur. I. 2. 121. IV. 28. Treitsch. X. 1. 79. Swartz Sv. Zool. I. Lảft. 3. n. 4. Zett. Ins. Lapp. 905. 1. God et Dup. Lepid. I. 38. pl. 1. 1. 2. II-S. Toxt. I. 140. Amaryssus llalm. Pap. Sv. 85. 1.

Fiärilen, fom i ôjriga Çuropa förefommer temligen allmän เฉå

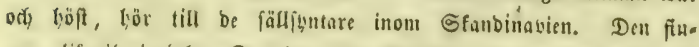
nes lifuat i bela Gmerige ort Rorige from be fboligape till

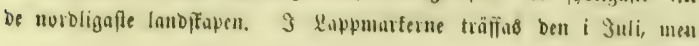




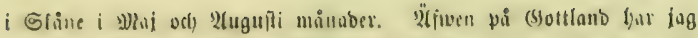

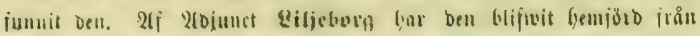
3) Irdangel.

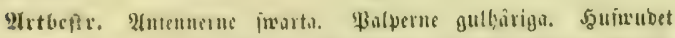
od) ruggfälbon fwartlubna med menue, på bell febuare i brebo tilltaganbe, guln firmmor. 2tobomen ofwan furars, umbertill ods Whi fioorme gul, med ett fusart fired längb glwarbera of be ied=

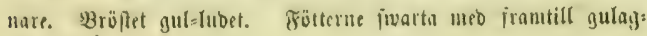
liga tibier.

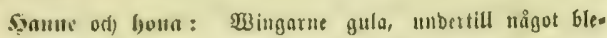
fare, med jiratta nerver. De fränte of̣man wio bafen utát bie ffru od) $i$ utfanten jurtis med gulagtiga ntomer; wa jebnare păllet Hubba med gutla balfuninformiga cller obala päafar. I wingivelifn emcllan 8 od) 9 nerverne finmes en fwart, meb ute

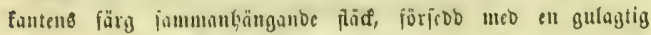
Flamma, orly i biffen surenue anbra, ben inbre fommanbe från framfanten od) gående twers öfuer biffältet, ben sttre utanjör Detas twerners ed ef Ginnande till framfanten. Bafwingarne ofwan frả roten längs inbre funten jwartagriga od $i$ utfanten urlioda meb ett brebt fwart banb, fywilfet inat bär ell rab ge" nom bopabe thagtiga atomer bilbabe fläfar och utát en rab af ambra balimninformign od) guls. Ejelima utfunten är gul o(t) $i$ astalbörnet funnco en frörre röbgul, rumbab fiăd, fom är $i$

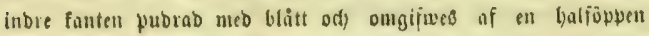
furart ring. Frammingarme äro unbertill tefnade fájom på öfre fiban, men be jurarta fäfarme ody banben äro tätare fubrabe med gult, buarigenom be ignas matta. Så är äpwen förbais lanbet med bofningarme, ber lifwall i utfantens fmarta fär? elle

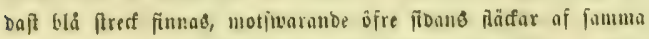
färg, oda be gula balfuninformiga fläfarme äto fiörre. Bat= scingarnes ficertlifa förlängning, fom fumbom är ni̊gos längre, fundom nigot fortare, är pai luảba fibor om ben beri utlöfanbe

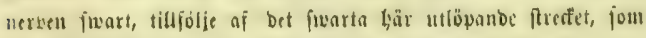
fafo̊t begrämar de gula fläfarme i utfanteno jurata färg. 


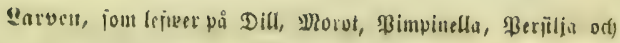

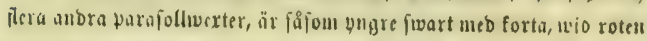

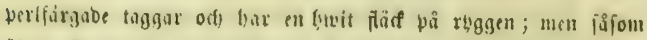

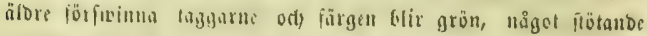
i Glitt, med fisurta ringur, probde a röbbruna punfter. \$ou.

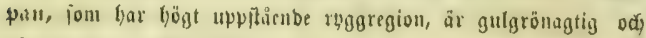

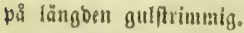

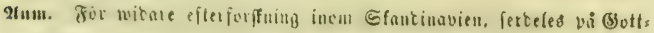

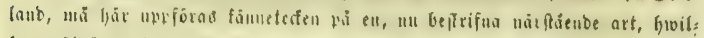

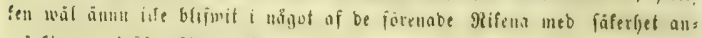

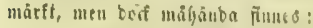

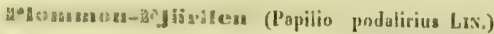

Alis Jlavis, utritaque nigro fasciatis; posticis supro ad aogulum ani macula nigra, cocrulco adopera et fulvo ciscumcincta,

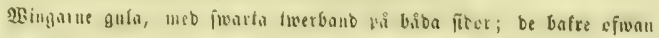

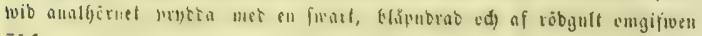
กีลลี่t.

Syn Paplo podalirius Lix, S. N. I. 2, 751, 36. Fann. E. S. III. 1. 24. 74. Honx. Pap. 1. 77. 1. 388, 390. Ocns, Sclun. 1. 2. 118. IY, 28.

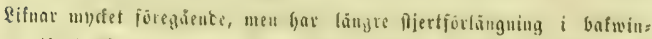

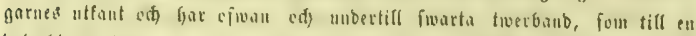

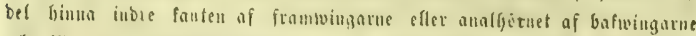
od till en bel swbora tefiforiman. I bofubingartes froathatign nufant

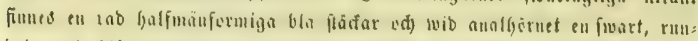

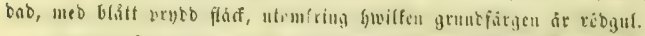

garveu, fom lefuer la itfilliga fruftröt, fifou silvile=, ظärus,

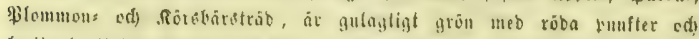

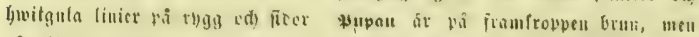

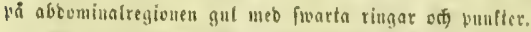

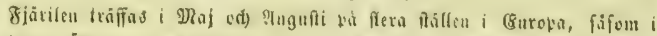

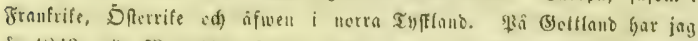

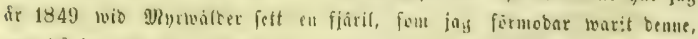
men bf jag ofe funte benägriga mig genem, fan jog ingenting befamtt berem nfgorn; Guarfice ing mes mu lemunte forta beffrifning blott refat

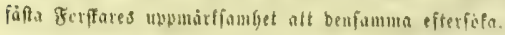




\section{Trib. Lyeanoida.}

Pedes 6 gresorii ; antici maris reliquis breviores, fominn famen omnes inter so conformes et æqualos.

Cellula discoidalis alarum aperta, costa transvorsa tantum spalia, tonuissima, clausa.

Margo anticus alarum anticarum costis quatuor aut simplicibus, itut una biramosa, (rarius triramosa) nempe costis ale 7-10, 1. 6-9 extonditur.

Costa alarum posticarum 9: 1:ma o trunco communi cum 2-4 radice simplice egrediens, ramulum nullum in lobum bisalum omiltens; 8-9 iterum o basi egredientes. Cost anticarum 10, ad basin inter se xqualos, rarius tantum 9 : 1:ma e hasi, 6:ta biramosa, (rarius triramosa), aut simplex.

Alse postic $x$ pro receptione abdominis canaliculalie, in margino exteriori aut catdatæ, aut dentatæ, aut integræ.

Larra foro ovalis, asollitormis capito rutractili, pedibusquo brevibus.

Pupa brevis, oblusa, apico anali ot filo por medium transverso alligata, rarius libera in terra quiescons.

Fütteruc 6 , gångfôtter; främfta puret hos bammen fortare än be öirige, men Gos bonan alla finßemellan lifiormiga ody lifa långa.

Diffrältet på wingarme öppet, blost flutet genom an ganfta fin, falft twernerv.

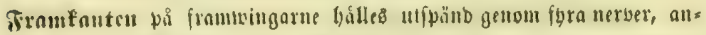
tingen alla enfla, eller orf bell ena twågrenig, fällan tregrenig; nemigen genom 7:be-10:be eller 6:te-9:be wingnerwerne.

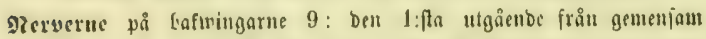
ftam meb ben 2-4, wib bajen enfel ods utfänbanbe ingen gren åt rotloten; 8-9 âter ugăende från tạch. Jramningarmes

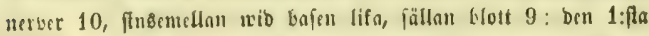
frôn bafen; Den 6:te twågrenig, (fällant tregrenig) eller enfel.

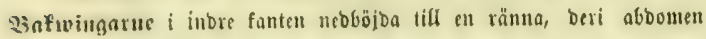
upptages; $i$ yttre fanten antingen filertabe, tanbabe eller beln. 
Enrven năftan obal, afellformig mé inbrngbart buftwio od forta fötter.

\$tupan fort, trubgig, faftianganbe wib anus ody mebelf ent om

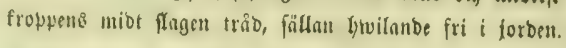

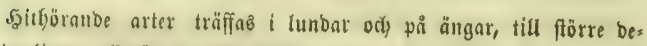
len temligen alluänn od) lyafwa bels twenne bele blott en ge-

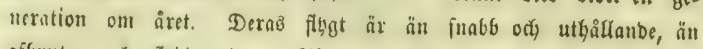
aforuten od flaboranbe. Såom larwer angripa några få trä= bene odi) luffermes blab, men de liefta leftwa på lägre bicotoledona werter.

(5sutppen fönberfaller $\mathrm{i}$ fölinnde fora flägten :

A. Alæ posticæ caudatæ.

Baftwingarne med ftientlif förlängning $i$ bttre fanten.

1. Costa sexta alarum anticarum triramosa.) Frantwingarneb 6:te ners tregtenig.

Zephyrus.

2. Costa sexta alarum anticarum simplex.) Franmingarnes 6:te nerv enfel (utan grenar). Thecla.

B. Alæ postice dentatæ. Costa sexta, simplex, et soptima, biramosa, alarum anticarum o loco oodem collula discoidalis ogredientes, rarissime in trunco brevi conjuncte.

Bativingarne i utfanten tunbabe. Den 6:te, enfla, od̆ ben 7:be twågreniga nerven på frams tvingarne utgående från famma fẩlle af bifffäl. tet, fällan förenabe med lywaranora $i$ en fort ftant.

Polyommatus.

C. Alæ posticœ integræ. Costa 6, simplex, et septimo, biramosa, alarum anticarum o locis diversis cellula discoidalis egrediontes.

Bufuingarne $i$ utfanten beloräbdabe. Den 6:te, Lyesena. enfla, od ben 7:be twaigreniga nerven på frams wingarne utgåsnde frân ftiloa fällen of biffeältct., 


\section{Slägtet: Rephyrus. (Dam.)}

(Polyommatus. Latr. Hesperia el Thecla. Fabir. Lyciena. Ocas.

\section{Zephyrus DaLy, ex, parte.)}

Antenux longiores, extrorsum sonsim crassioros.

Palpi squamati, inlra broviter ac parco pilosi, apicibus nutantihus; triarticulati. Articulus medius roliquis conjunctis Iongior, hasali latitudine aqualis, sed vorsus insertionom articuli ultimi nonibil allemulus; ultimus subnudus, basali brovior, apicom versus tenuior; fere conicus.

0cull hirti.

Pedes antici maris reliquis nonihil brovioros, fominæ tamen illis xqquales; cemora infra dense pilosa ; tibix pedum anticorum inermes, fed roliquorum calcaribus duobus validis ad insertionem tarsorum armate; tarsi pilis longis dentiformibus infrie instructi, antici maris, ut videlur, guadriarticulati, reliqui ut etiam omnes fomina quinqquarticulati : articulus primus tibiis gracilior, roliquis conjunctis longitudine oqualis, ceteri inter se xquales.

Ala postica ad marginom interiorem prope angulum ani oxcis $x$, in margino exterioro caudato.

Coste alarum posticarum $9: 1-3$ o trunco communi suporiori, 4 o costula spuria collula, :5-7 a Irunco communi inforiori, 8-9 e basi egrediontos. Coste anticarum 9: 1 c hasi, 24 o trunco communi inforiori, 3 o costula transversa spuria, 6-- 8 o Irunco communi suporiori, 9 itorum o hasi ogredientes; 6:ta triramosa.

2(utcuncrue temligen li̊nga, utît fmåningom tilltiofnande.

Şalperue fiälliga, unbertill fort od) glept lubna, mé̉ lutanbe fpete jar, trelcbabc; medlerfla leben längre än be öfrige tifljammans tagna, lifa bred med basleden, men mot ben fiftas lebiognistg nâgot afimaluanbe; fịta leben fortare än vasleben, nâftan na*

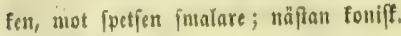

Ëbueul lubna.

Fötteruc: be främic bos bannen nagot fortare än be öjriga, men 


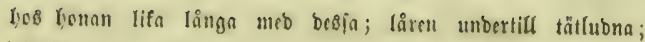
frâmfti parets tibier otwäpnabe, men be sfrigas beräpuabe meb 2: the ftarfa footrat wid lebfogningen med torjen; tarferme un= bertill förfroba med linga, tanbformiga bår; be främre lyos haus nen, filfout bet funes, furlebabe, men be öfriga od alla bos lio.

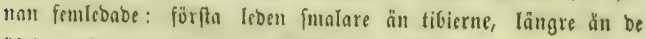
öfriga tillammantagna; be ofriga fimsemellan lifa.

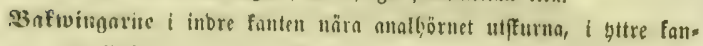
ten förfebda meb ftiertlif förlängning.

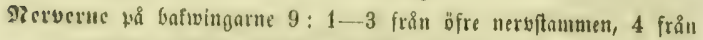

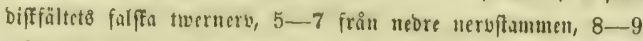
frål bafm. Sramuingarnes uerver $9: 1$ :fta från bafen, $2-4$ frin nebre nerbftsmunen, 5 från ben jaffa tmernerben, $6-8$ fro̊n öfre nersptanmen, 9:be iter frin bafen; Den 6:te tregrenig. De twenne tiff betta fägte lyörande Sfanbinavifa arterne wiftas

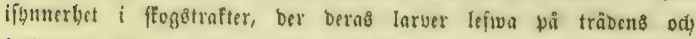
Guftarmes töf. Earverne äro afellormiga, o. tw. i. aflangt ägg= runba med fwälio rugs ods mot fiborne utplattabe, fi̊ att föt-

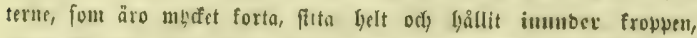
längs fidorne. S208 betta flägte äro be mot anus afîmalnambe och fålebes fuebare framull mot hufmubet, fon fan inbragas $i$ be förfa fropparingasue. Siroppen är betäcfi med forta bår ody hax minbre

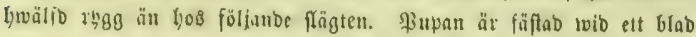
ody är umbertill platt, men thi rhggen ganffa buälfb, Bjiverallt ni= got lyảrig, uäftan femmtiort odly nutio utan taggar.

B. Eikc-smabbvingen (Zophyrus quercus lin.)

Alis infra cinoreis striga alba, intus obscuro marginata, of ad angulum ani posticarum marula gomina fulva. L. al. exp. $30-35$ millim.

Mas : alis supra fuscis, cyaneo micantibus, marginibus nigris.

Femina: alis supra fuscis; anticis disco coeruleis.

Wingarme unbertill affegri meb en butit, ina mörf fantab

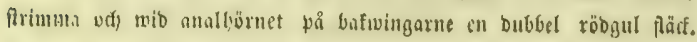
ฉ. e. wingip. $1 \frac{1}{4}-1 \frac{\gamma}{16}$ \&. 


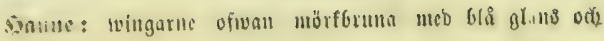
jwarti fauter.

Syoun: wingurne ojwan mörforma, be främu $\mathrm{i}$ biffen 6 lå.

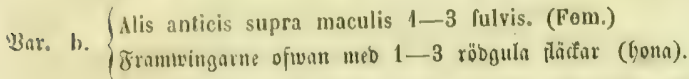

Syn. Pap. quercus Lï. L. Sv. 1072. Ilesperia Habr. E. S. III. 1. 278. 72. Papilio Ilïbn. Pap. 1. 73, f, 36-

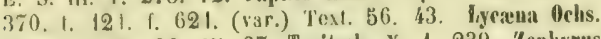
Schm, 1. 2. 96. IV. 27. Treitsch. X. 1. 239. Zephyrus Dalın. P'. Sv. 90. 1. Polyommatus God. of Bup. Inpid. I. 190. pl. 9 secund. ஓ. 1. pl, 9 tert. f. 3. Thecla II-S. Toxt. 1. 137. Gerhard Lyeænen. pl. 3. f. 3.

Denna urafra art trïfias uti efeftog $i$ föbra ody mederfta Gives

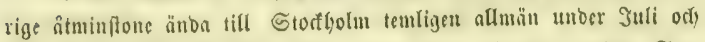
2lugufti minnoer, ftunoom ofjä, ebura mera fälignt, unber Sepo

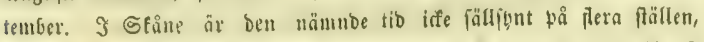

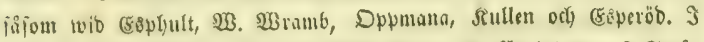

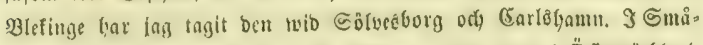

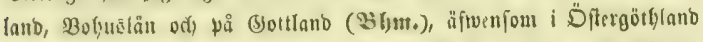
(Etcub.) ār ben ber ody lytwar talrif. Rifajå omifring Stodfolm. Norbligare in bet mig iffe befant, att ben förcfonmer, ods inom Rorige är Deณ ännu ef ammätft.

2rtbepre. 2tutennerme juata meb Gruita ringar. Rlubfan ofman

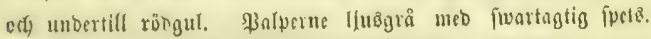

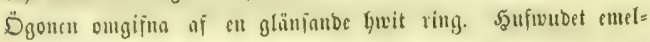
lan ögonem o(h) under antennerne blåagtigt grätt med tuenne fivanta frect, för öfrigt orunlubet äfwenfon ruggföloen. 216. bomen ofunan jwarterun, unbertill grågtig. Böjtet lyvit= grått. Fötterue Gwitgri, ber of̧ flwar frötanbe $i$ gult. Tar= ierne fwartringlabe. Bafwingarne med en imal, flertlif förs

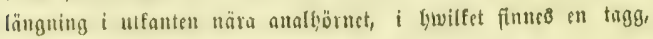
bildab af vingen ợ ett tätare bopande af franfat.

Snnuc: Bingarne oftuan juartbruma med mỏrfblâ glang, fom ät met elter minbre farf och toblig ollt efter bagern. Eramuingarme blont $i$ utfunten, utcu Gnfwingarnc i alla fanterue 
temligen frcot furta. 2Bingarne unbertill affegrá med an, if

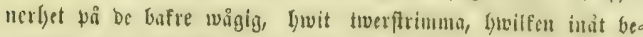
gränjas of ell mörforuแ cller jwartagtig ffuggning ody pa fram= wingarne förfwinner innan ben lyunnit infunten. Rängs utfan= terme löper en rab of otubliga, lywitagtiga, inüt mörffuggabe,

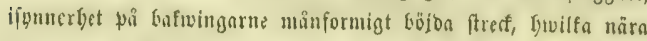
frnumingarnes bafre börn gränfa intill mörforuna, utit ftun=

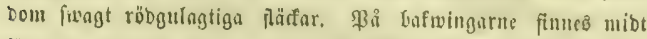
för bitt fifertlifa iörläugningen en röbgul fläct, fom i mibten yar en fwart funft; $i$ annlförnet fitmes en amtan of faumua

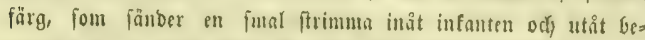

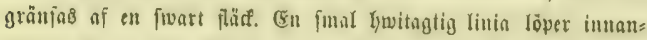

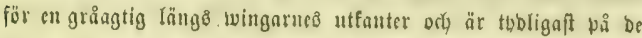
bafre. Eranfarme airs ofwan wid roten brunagtiga, $i$ fpetfen

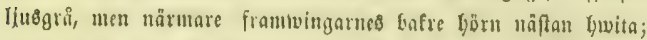
unbertill äro be aftegrå.

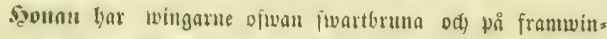

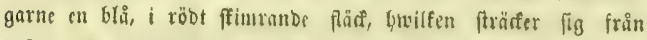

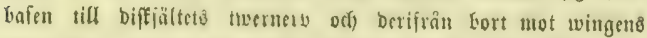
Gafre börn, fwwilfet den lifwäl ife upplyimer, fant fölfer tvin=

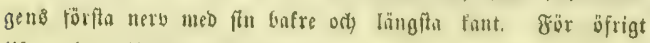
lifuar bon till färgen Gamuen.

Barveu, fom $i$ bördan of Juni träffas jullumutu pai efent UTab, är breb, något platt, Grunagtig meb ell rab upwrölda, trefantiga, senom en fiwart linta belabe, gutla fä̆far, fount leb= afbelningar of famma färg. Saufwubet är rumbabt oc) brunt, men be bafue fropperingarme oftaft gulagtiga. şupan ät tun. bab, på mibten tlorfare, frun ucb ljuiare fläfar.

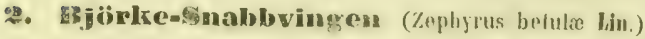

Mis infra luteis; anticis macula fusea ad costulam transvorsam cellule ol fascia lulva postica; posticis fascia fulva utringue albo marginata. L. al. exp. $3 i$ - $\$ 0$ millim. 
Mas : Alis supra fuscis; posticis maculis duobus fulvis in margine exteriori.

Femina : Alis supra luscis, anticis macula magna lutoofulva in disco; posticis ut in mare.

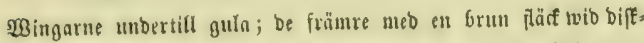
fältets twernerb odf ett röbgult tweetbanb nära utfanten; be bafre med

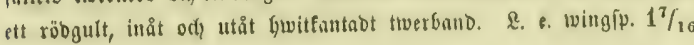
$-15 \%$ t.

Sanume: wingarne ofwan mürforuna; be Gafre med 2:me rōbgula fläđa $i$ utfanten.

Syoun: Framwingarne ofwan mörforuna med en-for guls

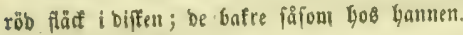

Sjn. Pap. be/ula Lin. F. Sv. 1070. Hï̈bn. P’ap. 1. 76. 383-38\%. Toxt. 58. 5I. Ilesperia Vabr. E. S. III. 1. 277. 69. Lyeana 0chs. Schm. I. 2. 113. IV. 28. Treitsch. X. 1. 79. 240. Zephyrus Malm. 1'ap. Sv. 90. 2. Polyoumatus God. ef Depl. Lepid. I. 181. Pl. 9. I. 1. Thecla Iett. Ins. Lapp. 909. 1. II-S. Text. I. 137. Gerh. Lycænen. pl. 1. f, 1 .

Riffom föregácnbe iürefonumer äfwen Denna art i foggetrafterne

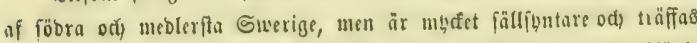

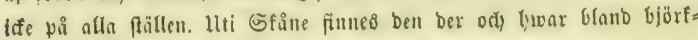
จ() flinbuffax, någun gång äfwen $i$ träbgårbar. 3 Blefinge bas

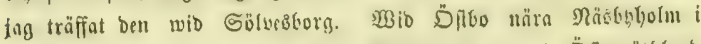

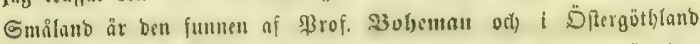

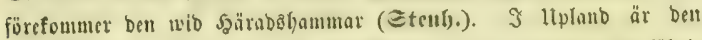

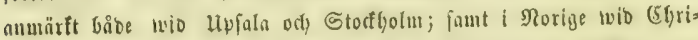

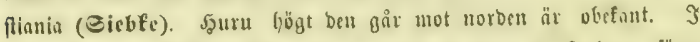
norra belen af Weflumanland och $i$ ben föbri belen af Dalarue föres fonmer ben ef (Frcorid)i.), men \$rof. Betterfteot anjör ben få

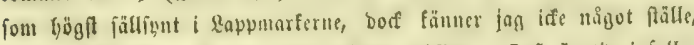

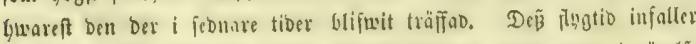

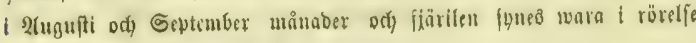
enbaft några timmar twib mibbagstiben. 


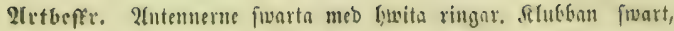
$i$ fwetjen röogul. Falperne lywita, $i$ fpelfen juata. D̈gonen oungifna af enl gmit sing. Şufwubet med röborun fubd, famit antyoning till twenne fwatta linist emellan ögonen. Ilyorax brunluber. 2(6bomen of wan brum, unbertill lyritagtig. Bröpet

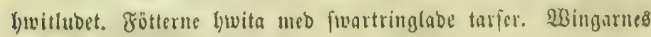
form fåfom yos föregående.

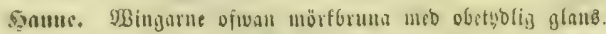
De främte med ett fwartagrigt ftrect wio biffiäteto fllut ocf ell liujare, i röbgult fiötanto, otyblig fäa berutaniỏs. De bafie

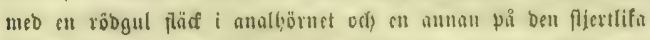
förlăıgningen. Ilnbertill äro wingurme twafert gula, fötande $i$

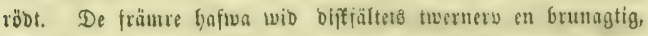

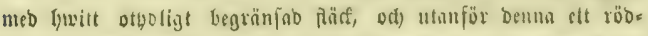
gult eller rofffärgabt banb, fon wid iramtanten är brebaft odf Gafåt flutar i en jpets inuan det bunnit intanten, jamt utât fes= gränfas of en l)wit o(1) en mörforan linie, men inåt af

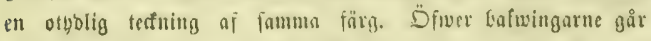
offiâ ett röbgult aller roffiärgnot band, lywars bába fibor tee

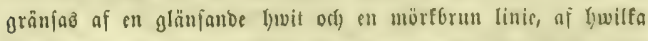

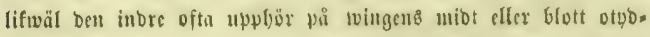
lint portättes mot infanten. Framiarne ofman od) mbertifl Llwita, men pis safivingarmes unbre fiba, framout ben pitertlifa

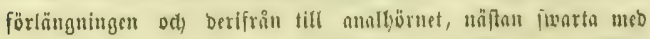
blott forta brwita fpetiar.

syoun lifuar bammen, mes har få fromevingarne en flor, gul, i röbt ftötnnde Rä̊f, quvilfen, belïgen utanjör diffiälteto twerncrve firäder fog poi tweren lingt fofiâ utan att linnta

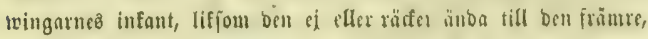
eด̆uru ben fommer närmare Denna, ën ben förra.

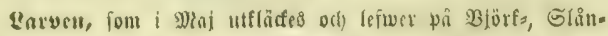

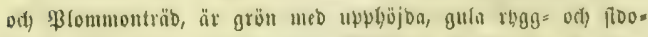

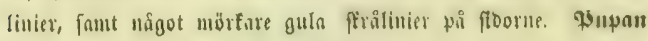
är brun med otb̧bliga, likfare ftrimumor. 


\section{Slägtet: Thecla $\left(\mathrm{F}_{\mathrm{ABn}}\right.$.)}

(Polyonmatus Latr. Hesperia Fabr, Zephyrus Das.3.

\section{Lycæna Ocus.)}

Antemm longiores, extrorsum incrassatx, clavam elongatam, cylindricam, baud determinatam constituentos.

Palpi *) squamati, inlra plus minus pilosi, apicibus porroctis I. subadscendontibus, triarticulati. Articulus modius reliquis conjunetis haud longior, articulationem versus ut in gonoro præcedente gracilior; ullimus subnudus, basali aut longiludine xqualis aut eo longior, gracilis, latitudine conformis.

\section{Ocull hirti.}

Pedes ut in genere precodonte, sod articulus socundus tarsorum, presortim posticorum, tortio, hic quarto, minuto, longior, ot quintus secundo rqualis.

Ala ut in genere precedente.

Costa alarum posticarum omnino 11 in gonero precodonte; anticarum 10: 1 o basi, 2-4 o trunco communi inferiori, 5 e costula transversa spuria, 6-9 o trunco communi superiori, 10 iterum o basi ogrodientes, 6-7 simplicns, ex eodem Joco, angulo antico, collule discoidalis ogrodiontes.

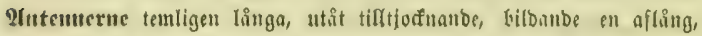
cblinbrift, füga affetlo flubbr.

Salperne *) ftällign, unbertifl mer eller minore Iubna, mé iramftiende eller något uppåtfiganbe jpetfar, trelebabe: meblerfta leben föga lângre ån be öfriga tifl fammantugna, mot lebfogningen, fåjom

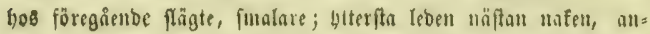
tingen Jifa lång med faß̉leben eller längre än benua, fmal, till bela erebben lifiormig.

Sgonen Jubna.

") Tabula anatomiea palporum artieulorum proportionem extibens:

1. Articulus ultimus basali longior: Th. lynceus it album.

2 Articulus ultimus basuli longitudine æqualis: Th pruni et rubi. 


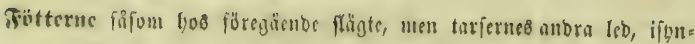
nerlyet pă be Gafre, längre än ben 3:bje od benna längre ău Den 4:be, four är minft; ben 5:te lifa uteo ben 2:bra.

233ingarue fịfom

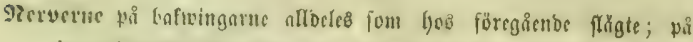
frambingntne 10: ocn 1:fta froin bajen, 2-4 froin nedre nerv= ftammen, 5:te frin ben falffa twernerwen, 6-9 från öfre nerbs ftammen, 10:be iter frin fufen; 0:te od) 7:be utgå från fammu flälle of biffältet, nemligen frion bef främre börn, od äro enfla (utan grenar).

Detta flägte̊ arter bafwa famun lefnabsfätt fom föregåenbes ; men berns larver äro nigot fmalare bafât od) pupor autingen fmalure mot amus ofl) flätn effer of nigot taggign odi) fantiga.

\section{耳. Berneke-fiasbbvingen (Thorla lyncous Fabr.)}

Alis infra fuscis striga lunata alba, oxtus sinuata, in anticis obsoleta; posticis maculis marginalibus disjunctis fulvis. L. al. exp, 35-40 millim.

Mas: alis supra fuceis macula fulva ad angulum ani.

Femina: alis supra fuscis; anticis Inacula fulva indelorminata.

TBingarue unbertial bruna meb en fimit frimmsa, beftiente of

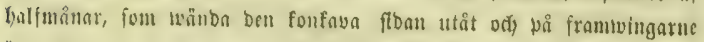
äro otgoliģn; be bafre meb ffilbn röbgula flăcfar $i$ utfanten. \&. e. wingip. $1 \frac{\gamma}{16}-1 \frac{5}{8}$ t.

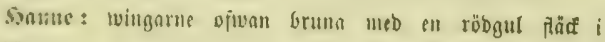
smalförtts.

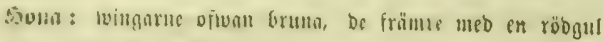

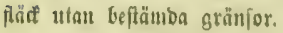

Sỵ. Ilesperia lynceus laltr. E. S. III. 1. 276, 73. Pap. ilicis Esp. Schum. 1. 1. 39. Sp. 13. 1. 1. b. p. 353. Iycena Ochs. Sclum. I. 2. $10 \%$. iv. 27. Ilibn, Pap. I. 7i.). 378. 379. Texl. 57. 47. Tephyrus Dalm. P. Sv. 94. 5. Theela II-S. Toxt. I. 136. Polyommatus lynceus God. ot Bup. Leprid. 1. 186. pl. 9 tort. 1. 1. Gerh. Lycrenon. t. 2. f. 2. 


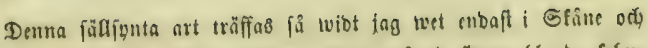

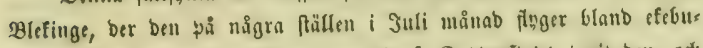

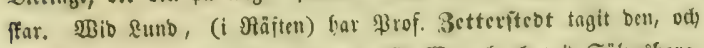
jag Gar träffat ben temligen talrif wio $\mathfrak{W}$. 2Bramb od) trid Sölueşborg.

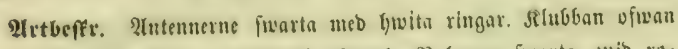
fwart, $i$ fpetfen od unbertill röbgul. Balperne furata, wio ros ten od) pá inbre fiban buvitagtiga. D̈gonen omgifna of en

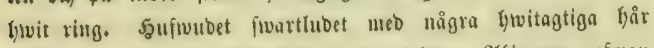

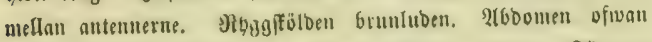
brun, unbertill gråagtig. Bröftet gråblâtt, lubct. Fötterne fonitgrå, med fwartringlabe tarfer. Bafwingarne $i$ Gafre tanten förebba meo en fort flectlif förlängning.

sanume. S3ingarme ofwan mörfbruma; be bafre med en

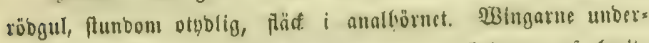
till bruna, braganbe i röbt, prtybda meb en frimuna af fumita

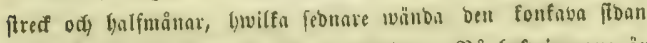
utît od äro pơ frammingarne otybliga. Bî bafningarme ât besna frrimma mudet wågig mot infanten, od) wib utfanten finme en rab röbgula fäfar, fom inåt begränfas lowarbera of en fivart bulfbåge; $i$ fielfion utfanten af famma wingar gå $\mathrm{en}$ Givit linie. Sramwingarme äro svlo bajen $i$ fränre fanten funalt gulagtiga. Eranjarme oftwan od) unbertill brunn, men på baf= twingarnes Pltertlifa förlängning od) Derifrion till anallyörnet Guitagtiga, blanbabt wed fivart.

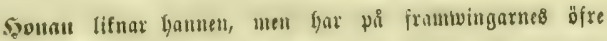
fito en röbgul flăf, fom belno genom be mörfa twingnerverne och inåt förlorar fto 1 grunbfärgen.

Sarven är blefgrön med $3:$ ane afbrutua gula liuler, en

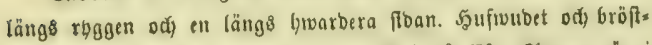
fötterne äro fwarta. Den lefwer $\mathrm{i}$ \laj på (Ef. \$upan itr $i$ börłan gulagtig, feban fruแ mcb 3:ue raber mörfa punfter på aloominaltegionsu. 
2. Ane-Snabbvingen (Thecla W album Knoch.)

Alis infra fuscis, striga alba, in posticis W album monlionto; posticis fascia aurantia marginali. L. al. oxp. $30-35$ millim.

Mas, of Femina: alis supra fuscis, posticis macula fulva ad angulum ani.

WBingarme unbertill bruma meb en breit frimma, fom på be Gufre lifuar ett $\mathrm{W}$; De bafre befuton mid ett orangefärgabt twerband i utfanten. \&. ค. wingip. $1 \frac{1}{4}-1 \frac{1}{16}$ t.

scaute od) boua: WBingante ofwan bruna; be batre med en röbgul fläđ $i$ analgôrruet.

Syn. Pap. IV album Kuoch. Beitr. II. 85. t. 6. f. 1. 2. Ilübn. Pap. t. 75. f. 380.381 . Text. 58. 49. Lyeaua Nclis. Schm. I. 2. 109. IV. 28. Zephyrus Dalm. Paj). Śv. 91. 4. Polyommatus (iod. et Dup. Lepid. I. 188.11 . 9. f. 3. pl. 9. Lort. f. 2. Theela Zett. Ins. Lapp. 909.2. II-S, Text. I, 136, Gerh. Lycanen, t. 1. f. 3.

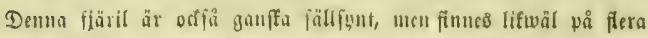

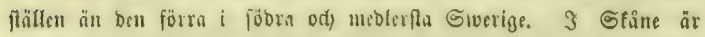
Den funmen talritt i \$arndislşfan wib \&unD (3ett.). \$ llplanb

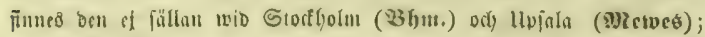

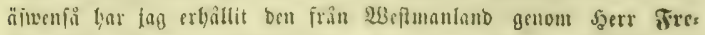
oridjion. Detta är äfwen für anten ben norbligafte lofal inom

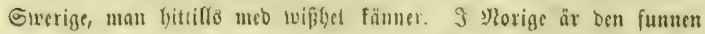

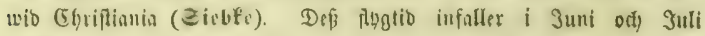
månaber.

Qretbefit. 2Intenner od) öriga fropwsbelar fäfom fos föres gåenbe nrt, men brötet är frunlubet ofl) bafwingarne bafwa

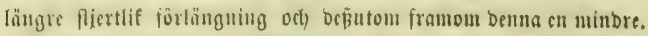

syame od) houn. Wingarme ofwan fwartbruna; be Gafre

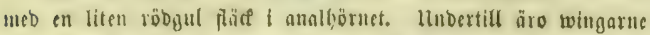
Grunn, fütande $i$ röbt, med ell buvit tuverftrimma, Gywilfen på be

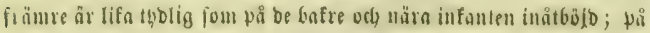

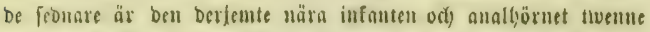
gåuger ftatpt winflab, få att ben lifuar ett bublelt latinf̧t W. 
Lăngs be balres utfant gầ ctt orangefärgabt tmetranb, fom af= fmalnar fromit očs inåt begränjas of en rab fwarta fyalfGâgur, faut năra anallyörnct har en Glåagtig oḑ länge utfanten in eller flera fivarta pläfor. Snom och uton betta twerbanb går.

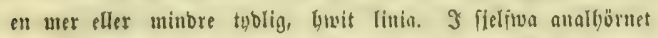
finnes en funart thäf. Franjarne fåịom hoo föregåenbe.

Sarveu, fon lefmer po̊ 2 flm (Ulmus compestrie), är grön

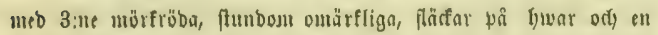
af be Gaferfa fropperingarne, faut fyar länga r thggen en bubbel rab af fpetfar. Närn förwanblingstiben Glir ben brumagtig.

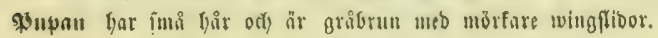

\section{Plommonmoghobingen (Thecla pruni Lin.)}

Alis inlra olivareo-fuscis striga alba; posticis lascia marginali fulva nigro punctata. L. al $\theta \times 1,30-35$ millim.

Mas: alis supra fuscis; posticis maculis marginalibus fulvis.

Femina: alis supra fuscis maculis marginalibus fulvis. SBingarne unbertill olibbruna meb on blvit ftrimma; be Gafre mib ett röbgult, jwart punfternot twerbant wib utfanten. $\mathfrak{l}$. $c$. ivingfp. $13 / 4-17 / 10$ t.

Syanue : wingarne ofwan bruna; de bafre med röbgula fื̉̆น̊a $i$ utfanten.

soma : wingarme vfwan bruma med röbgula fläcfar $i$ uts fanten.

Syn. Pap, pruni Lin, F, Sv, 1071, Nï̈bn, Рap, t. 76. 1. 386. 387. Toxt. 58. 50. Hesperia Falor. K. s. III. I. 277. 70. Lycsena Ochs, Schur. I. 2. 111. IV. 28. Theplyyrus Dalm. P'ap. Sv. 91. 3. Polyosmatus Giod. of Ilup. Lepid. I. 184. pl, 9. ケ. 2. Thecla II-S. Toxt. I. 136. Gerh. Lycenen. t. 1. f. 2.

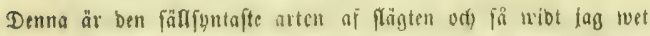
enbaft funten wib \&unb of \$rof. Betierfteot. Dep fugtio infafter

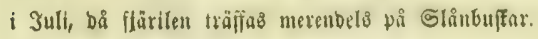


2(r'tbeffe. Sropyêbelarues fărg od) form lie föregåenbe art8; men benna lat brott ch pletertif fürlanngning $i$ baftuingarnes bttre fant.

Gaumc. WBingarme ojwan fiwartbruna; be bafre uted en

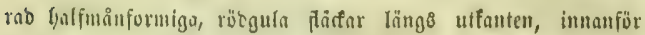

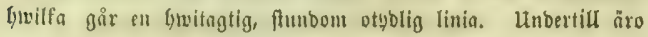
mingarne olivtruma meb en bwit, $i$ forta linier upwlöf frimma, fom inåt fegränfue af fwart ody är lifa tyblig Hå alla twingarne. Räแnุą ufanten af framtsingurne gåx en rab malta ody othbliga röbgula fläfar, of twilfa en odf aıman är ina förfedo med en fwart fläc, jom lifalebes inåt otydligt begränfos af Gtvitt. Rängื utfanten of Gafwingarme gåı ctt röbgult twerbanb, bwila fet är inis förjeot med runba, ody utåt meb trefantiga, jwarta

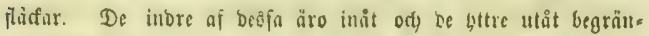

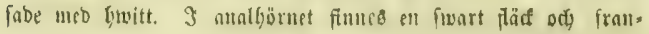
farne äro fåfom ुos föregående art.

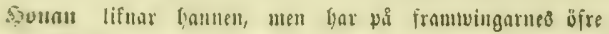
fiba nära fnfre hürmet några rübgula färfar, motiwaranoe bnf= wingarnte.

Qatweu, fom lejwet pá l'runus spinosa ody domostica,

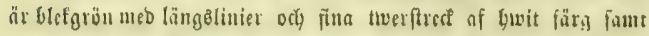
lex par forta, föttiga, jwartpunfterabe upprjöjningar pả rsggen. Def fufmud àr gult med tweme jwarta punfter. Wupan ä

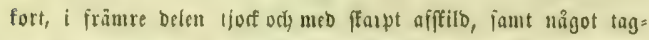
gig abbominalregion. Till järgen är ben mörfforun meb Ģtwita ledafidelningat ody Givitpunfterad lyufwubregion.

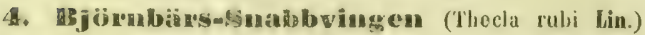

Alis infra viridibus, posticis aut unicoloribus aut punctis nonullis albis. L. al, exp. $25-30$ millim.

Mlas: alis supra olivacoo-fuscis, anticis loco oh-curo supra excavalo, infra convoxo.

Femina: alis supra olivacoo-luscis. 


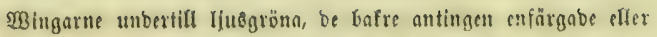
of förfeboe med några juwita funfter. \&. e. minglp. $1-1 \frac{1}{4}$ t.

Scanme: mingarne ofton olisbruma, be främure meb ctt mörft flälle, fom ofwan ầ urlyolfabt och unbertill fonwext.

Syour : wingarne ofwan olivbruna.

Syn. Pap. rubi Liu, F. Sv. 1077. Hübn. Pap. 1. 72. 1. 364. 365 . Text. 55, 41. Hesperia Vabr. E. S. III. 1. 257. 102. Lyeanat Mehs, Schum. I. 2. 94. IV. 27. Treitseh. X. 1. 77. Zett Ins. Lapp. 910. 1. Zephyrus Ihalm. Pap. Sv. 94. 12. Polyommatus Goul. ot Dup. Lopid. I. 206. pl. 10. I. 3. pl. 10 secund. . B. Thecla II-S. Text. 1. 135. Ferh. Lycenen. 1. 3. 6. 2.

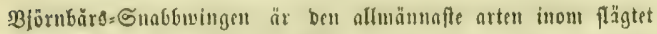

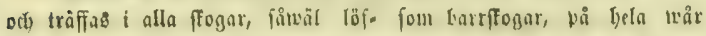
fyali $=0$, be norbligafte trafterme iffe unbantagna. Defis fungtio infafler i Maí ợ sutuli manaber.

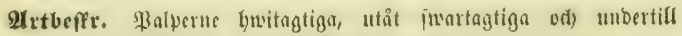

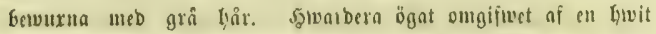

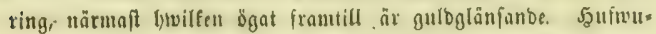
bet brunbårigt; melon nutemuerne ăto liftuăl hairen grågtign. Stntennerne fwarta med liwita ringar. Slubsan $i$ jetien töbs

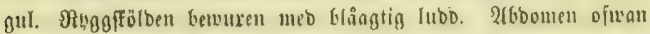
Grun, unbertifl grönngtig. Bröftet grålubet. F̧ötserne blägrås agtiga med fwartringlabe tarjer.

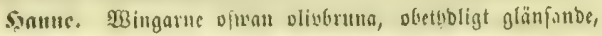

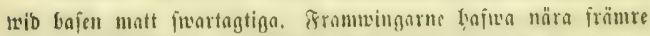
fanten wio biffältet elt mörfare flüc, fom ofwantill är iörbjus pab ody undertill ubpröido. $₹$ annlfiomet of bafuingarne finmes fiunbom en otholig gulagtig fläs ofl) fronjarme, fom för öjrigt fyafura famma färg fon suingarme, äro byä lyvitagtiga. Lfuber:

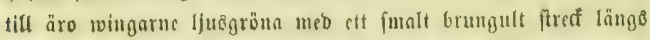
utfanterne ody bylif järgläggning $i$ framfonten of framuingarne. Bafwingarne bajma $i$ analtürnet (II liealt fläf od) ufta $i$ frams fanten en lywit punft, lywilfen frunoom är âffüljo af en tho bus= lifa twero ofwer winzen, neแ ftunbom äfwen lafuns. Sällan 


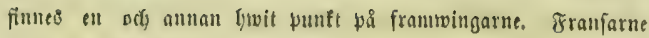

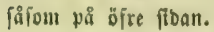

Syounu, fom lifunr bannen, fafnar bet omuảmuba urlyolfabe fället på frumbingarne.

Earveu är grägrön eller gulgro̊n mé butagtig rb̆gglinia,

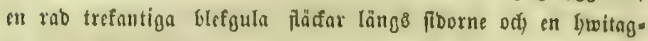
tig linia b̈fwer fotrabert. Den lefwer på Rubus fruticosus, Sarothamnus scoparius m. fl. anbra werter. \$upan, fom är

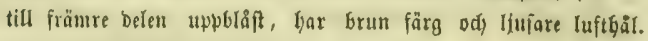
รูon öfwerwintrar.

\section{Sliggtet: Polyoumatus (LArr.)}

(Lycana Fabr. Ochs. Zett. Hesperia Farr. Zephyrus Dalm.)

Autemux longioros, clavatx; clava brevis, oblongo-ovata, crassa dolerminata.

Palpi spuamali, infra donse pilosi, apicibus porrectis $i$. subadscondentibus, triarticulati. Articulus medius roliquis conjunclis longior; basalis minutus; ultimus basali multo longior, gracilis, attonuatus, apice acuto.

Nculi nudi.

Pelles ut in genoro prxcedente, sed articulus tarsorum primus tibiis vix gracilior, rarius in posticis is distincto crassior ot articulis roliquis conjunctis longior.

Alæ posticx ut in genere pricedento, sed in margino postoriori tantum dentate.

Costre alarum posticarum ul. in prxcedentibus; anticarum $10 \mathrm{ut}$ in genere precedonto egrodientes, sed costa septima biramosa*). Costia sexta ot septima o loco oodem collula egrediuntur.

* Polyumalus virgaurea interdum costos tantum novem labet, quonian tune cosia 6 et 7 , ex endem loco cellula egredienles, in trunco brevi communi sunt conjunate. 


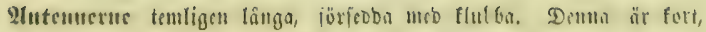
aịlångträggformig, tlorf och tholigt niffild frân ifaftet.

Polperus fjülliga, unbertill tätt lubma, mo framftienoc cller uppaits Pliganbe fpetfar, trelebnoe: meblerfta leben längre än be jiriga

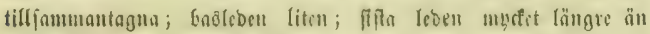
Gastebent, imal, ffarpt tillipetiab.

Şgonen natna.

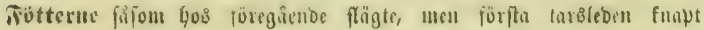

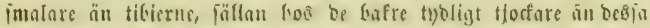
ody längre än be ofrige lebertie tll fammantagna.

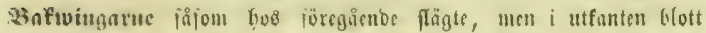
tanbabe.

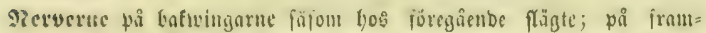

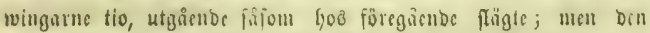
7:be är twågrenig. 6:te ợ 7:De netuerne utgâ frân fammแa

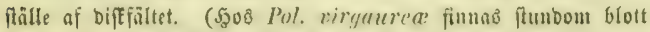

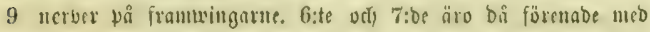
lwaranora till all fort ftam; men betta àr lifueñl iffe alltio för= båluanbet.)

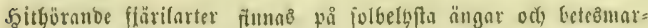

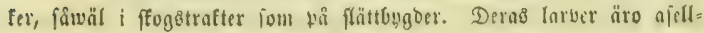
jormiga, men temligen längfträrfta o(h) på fiborne något fam=

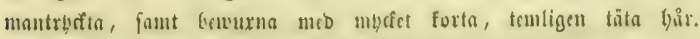
De lefima på låga wexter orl fömanblingen fer nära wib cllex

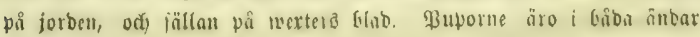
trubbiga.

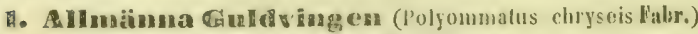

Alis in/ra cinurascentihus punctis ocellaribus numerosis niisris; posticis fascia intramarginali fulva, antice ovanosemuli, nigro-punctata. L. al. exp. 32-37 millim.

Mas: alis supra fulvis, aureo nitentibus marginilus nigris coeruloo micantiluss.

Femina : alis supra fulvescentibus plum minus infuscalis punctis nigris; posticis fascia intramarginali lulva. 
Wingarne unbertill affegråagtiga med talrifa fwarta ocell.lifa punfter; be bafre twid utfaitten propboa med ett töbgult, fmartpunfte. rabt tuverbanto, foum frantåt är förfwituanbe. \&. e. wingfp. $1 \frac{5}{10}$ $-1 \frac{1}{2}$ t.

Seaume : wingarme ofwan töbgula, gulbglïufande meb fwarta, $i$ blåt ftötanbe utfanter.

Şvun: wingarne ofwan röogulagtiga, net eller minbre ufrerbragna meo brunt, förfed a meb jwarta punfter; be bafre $i$ utfanten prabda meb ett röbgult twerband.

Sjn. Pap. chrysces WV. V. p. 181. Fam. H. n. 3. Hübn, Pap. t. 68. f. 337. 338. t. 74. f. 355. Text. 53. 44 Ilesperia Valır, E. S. III. 1.309. 174. Lycaena Ochs. Schm. I. 2. 79. IV. 27. Treitsch. X. 1. 7\%. Zett. Ins. Lapp. 910. 2. Zephyrus Dilm. P. Sv. 92. 7. IDolyommatus God. of Dnp. Lopid. I. 198. pl. 9 secund. f. i. pl. 10 secund. f. 2. II-S. T'ext. I. 132. Gerh. Ljexnen t. 7. 1. 4. (Pap. hippothoë Lin. F. Sv. 1046. (mas.) Esp. Schim. I. t. 22. f. 3. p. 292. t. 78. cont. 28. f. 5. p. 133. t. 100. cont. 55. f. 2. p. 38. var. Pap. euridice Ilorkh. Schm. I. 143. 270. II. 220.)

Obs. $P$. hippothoë Auct. non in Suecia est reporta, quare synongmiam Linnoi cilatam ad hane speciom pertinere credo. Descriptio, ab Illustr. Viro 1. c. data, nullam dilficultatem prabot. "Statura P. virgaureæ", ut in discriptione Limnoi dicitur, cum P. chrys. etian' magis quam cum P. hippoth. congruit.

Bar. b. Punctis ocollaribus inlra in lineas elongatas nigras vel in maculas difformes confluentihus.

De ocellslifa punfterna unbertiu jamutanfluma till langs. gåenbe linier eller flädar af olifa form.

Bar. c. Alis posticis angulo antico magis rotundato, supra infuscatis fascia intramarginali fulva nigro-punctata; anticis supra fulvis dilutioribus* ${ }^{*}$ ). liorma minor.

Bafwingarme med meta afrunbabt frambörn, ojwan of werbragne med frum ude phobda meb ett röbgult, fwarte

-) Alow anticos fulvoo, in llavedinem vertontes. 
punfterabt tiverbant $i$ utfanten; be främre ofiuan fiufare rōbgula. Minore form.

Syn. Polyoumatus var. Stiberi Gerh. I. c. pl. 36. 1. 1. (ut videtur.)

Denna art förefommer mer fller minbre allmänt Hă wår

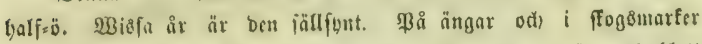

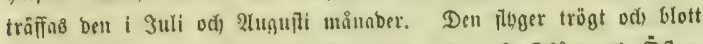

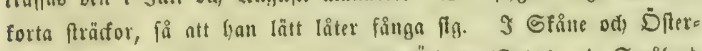

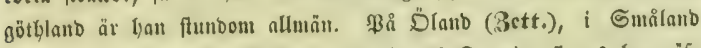
(36)m.) och $i$ be flefta ofriga provinfer of Swerige funnes bant, äfs wenfom i Norige. 23ax. b. förefoumer tilljamman méb wanliga formen men $23 a x$. c. thdes tillyöra be egentliga tergetrafterne. Den

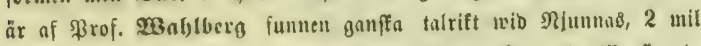

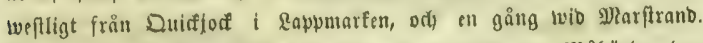

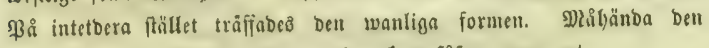

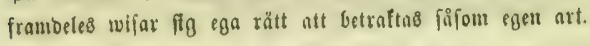

artbeffe. 2tutennetne fwarta med ywita ringar. Slubban i

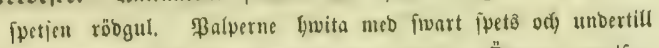
betuuxisa mé grå birr, blanbabe med fwarta. D̈gonen omgifna

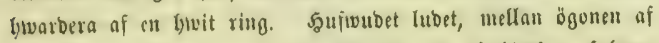
fwart, mellan antenuresne af gråagtig od bia bieşan af brun lubb. Nuggförben luben af fwartblatt, men på fulbrorne och balsfragen af grablått. 2tboomen ofwan fiwart meb gleja hår,

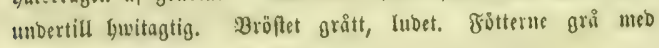
mörfa rebafdelningar på tarjernc.

Scanuc. W3ingarme ojwan röbgula, guloglänjanbe, mib bas jen, längs iramfantame od) utfanterne famt $i$ bafuingannes inbre

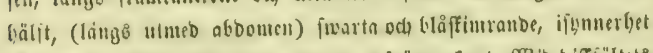

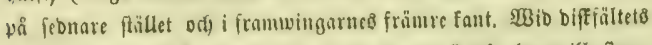

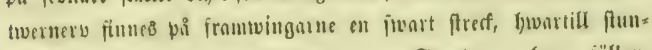

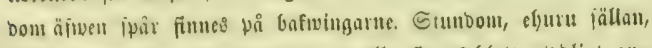

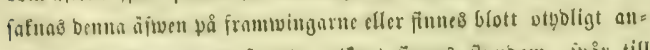
t)bo. I bafwingarnes fiwarta utfant fiunas funbom ipå till nágra rodgula päcfar. Llubertill äro เwingaıme mörfł affegrå , 


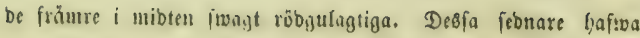
wid utfanten on rab otyoliga fwarta punftet, odis innanför benuta en astnan fiarft böld od) tholigare, lywars funfter ăro lywarbera ongifne of en bivit ring. 3 bifffältet fti̊ trenue ftörce punf. ter, parallelt meb franfanten vily lifalebes omgifna af broitt. \&ängs utfunten of lofwingarme giar ett röbgult med tnenne furarta, otybligare punftraber prybt banb, घwilfet lifwäl oftuft framint ăr förjwinnanbe, fî att bet är thbligaft nära analgörnet. Sunaniör deita gấ en rad thbligare fwarta puntter, Gwarbera

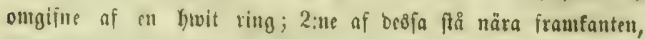
ffilda itån be jiriga; $i$ biffen od) nära bajen fintra flera fpribda uteb famma färg. (E) od) annan af beฮెja punfter jafnas flun= bom. Franfarne lyuita, unbertifl wib roten gråatiga.

Sous. Wingarne viwan fwartbruna med mer eller minbre röbgult $i$ biffen få framwingarne, Gwilfa derlemte bafwa wíb bifffälteţ flut ett fwart ftreç od närmare utfanten en twerrab frwarta wunfter, famt ftundom wib bafre bònet an förtan till ett orybligt röbgult Ganb. BBatwingarne, fom äro fwartbruna, lyafiva $i$ utfanten ett Grebt, töbgult twerbanb, h)wilfet förfwin. nct närmare frambörmet vतy är probot med fivarta eller mörf. Gruna, ofeftämba fläcist. Snnaujör betta bano innas afiwen funbom några $i$ tab prällba, bliagtiga punfter. \$å unbre fiban, fom för bfrigt lifnar bannena, äro frammingane $i$ mibten mera röbgula od) twerbandet $i$ bafwingarmes utfant thbligare.

23ax. b. Rifnar föregående, men bar ฟå Gafwingatnes unbre fiba i flillet för be jwarta punfterne bylifa fitrect eller trefans tiga, eller ơ annorlunba jormade, fätar. Det Gänber äfwen alt cremplar finuta, bos Givilta wingarme på ena fiban om frop.

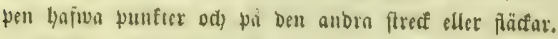

23ar. c. ñr minbre än Den normala formen. Seanuen bat

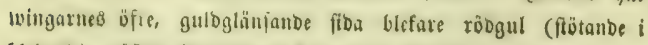

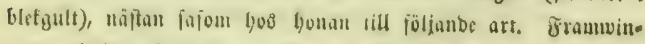

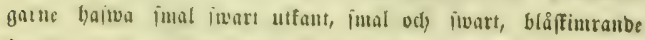
jramtant od ett fort jwat fored i biffen. Batwingarme àro 


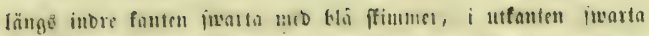
od) lyafwa Der at syoligt aiffilot röbguls, jwartiärfigt tmerband, iamt $i$ biffell ett jwart firter. Hubertill äro wingarue lifa bem

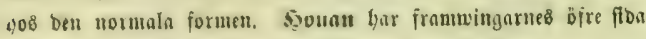
robogul, mio bafin od) längs fränire fanten - brunagtig, famt twenne twerraber fwarta punfer od en jrart läd $i$ biffen. Bafuingarne äro bruna med ett jurart fitect $i$ biffen od ett röbgult bano läıģ utfanten, Gurilfet är probot meo frarta punfter. Itnbertill lifna wingarme lyamens. Şonan till benna ba= restet lifnar, $i$ anjeende till ningarncs bire floa, $i$ baft lyonan till föllanbe art.

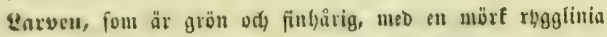
odh ljujare, buwita floftrimmor, lefwer bå attfilliga forearter (Rumex). \$upaı är äggformig, hysitgul meb fwarta ftrimmor

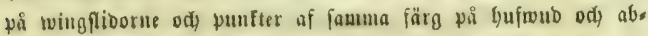
bouinalregion.

2. IRiftunge-Guldvingen (Polyommatu、 virgateæ lin.) Alis anticis infra fulvis punclis nigris; posticis infra fulrogrisois punctis nigris maculi que albic. Ł. al. oxp. 30-35 millim.

Mas: alis supra lulvo-aurois, immaculatis, margine nigro.

Femina: alis supra languidius lulvo-aurois, nigro punctalis ; posticis inluscatis.

Erammingarme unbertifl röbguls meo fnarta punfter; bafwins garme unbertill gufgrio med furnta punfter od) flägfar. \&. e. wing p. $1 \frac{1}{4}-1 \frac{7}{16}$

Scaunc. Wsingarme ofman röbgula, gulbglänfanibe, oplădabe, meb furarta utfanter.

Scour. WBingurue ojwan blefure röbgula, fwartwunfterabe ; be bafre ofwerbragna med brunt.

Syn. Pap. rirgaurea Lin. F. Sv. 1079. llïbn. P'ap. 1. 70. ศ. $349-354$. เ. 179. ศ. $884-887$ 'Toxt. 54. 36 Ilesperia Vahr. E. S. III. 4. 309. 173. Lyenena Ochs Sebm. I. 4. 85. IV. 87. Treitsch. X. 1. 74 . Zett. Ins. Lapp. 
910. 3. Zepluyrus Dalı. Pap. Sv. 92. \&. Polyommatus. God. ot Dup. Lepid. I. 202 pl. 9 secund. f. 6. pl. 10 socund. f. 4. II-S. Texi. I. 134. Gerh. Lyezenen 1. 5. f. 3. Polyontm. var, oranuln Gerb I. c. 1. 4.

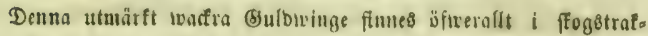
terne af föbra od meolerfa Smerige od Rorige, men furu lyögt ben egentligen piger mot norben är ämu oajgforbr. Dell norbligaple proving $i$ Stwerige, ter man wet att ben förefonmer är Dalorne. Brof. Setterftent anför ben iăom fällịnt i Rappmarferne, men utan

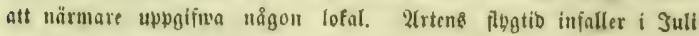

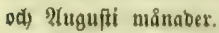

21tbeffs. 2(ntennerue oҺ beras flubba jåfou hos föregåenbe art. Balperne lywita, i ipetien fwarta odf unbertill bewuxna med

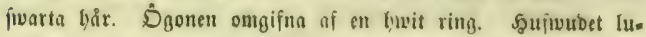
bet af röbgula bair, men emellan ögonen och unber antennerne

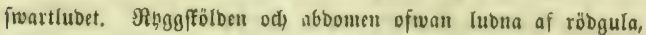
gulbglänfanbe bår. Den fonare unbertill britagtig. Bröptet G)wituligt. Fötterme fiwitagtiga; ti6ierne iramtill något ïatanbe i gult; tarferme fwatringlabe.

Б̄aute. WBingarne oiman glänjanbe gulbfärgabe, 1 utfane terne fivarta. Unbertill âro be främre röbgula, $i$ frnmifanten,

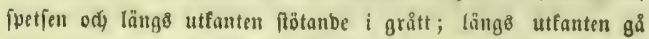
twenne raber fwnrta flădor eller punfter, af Givilfa ben optre är matt ody otmblig, od̆ i diffältet få parallelt med framfane ten trenue fworta päđơar. De bafre äro röbgulagtigt grå meb iprioba, fwartn, gulfantabe punfter $i$ biffen ơ närumnre utfan= ten en ofaumantyängande rab bulifa, till brwilf gränfa bywita

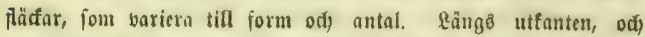

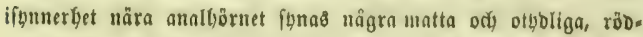
gula flïfar, guvilfa funbom $i$ mibten äro mürfffuggabe. Fran. jurme äro ofwan gulagtiga orf) unbertill foutande $i$ brumt.

sroual, fom unbertill lifnar haumen, nir till mingarnes ofre fiba, ifgnuerbet pa frantwingarne, bleft röbgul neb matt glamछ o(ด) har fmala, brunagtiga twingfanter. Nära utfanten af

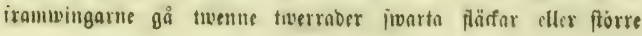


punfter, af hwilfa ben bttre är obeftämbare än ben inbre. $\rightrightarrows$ biffältet finnas befatom twemne fwarta flärfar, af lowilfa ben ftörre är belägen wib twernerven. Bafivingarne, fom äfwen äto röbgula, men fri̊n bajen till ftörre efler minbre utffrärçning o(t) futubom B̈wer bela ptan fuggnde meb brunt, Vafwa $i$ bifen en fwart flä odi) utanför bennn några twerraber frarta punt= ter, famt, bi wingarne äto alloelio offwerbragua med brunt, ett

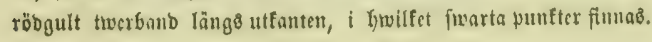

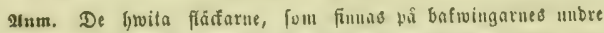
fiba, dato flere eller färre till autalet, flwarigenom ben obetybliga vas rieteten, forn Eerbarb unber uamu af oranula nfbilbat, uppifumer.

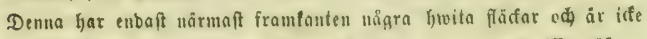
egenbounlig for norbligare belar af Gtanbinasien, utan träfias öfiver allt blanb ben normala formen.

Earven tifl benma art är mörfgrön med ell gul linia längs

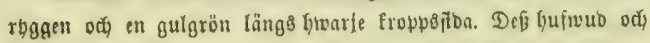
bröfffötter äro fwarta. Den lefwer på wantiga Räftumgan (Solidago virgaurea) ợ på åtftilliga Rumox-arter. \$upau år gulbrun meb mörfare mingfitbor.

\section{Syre-Guldvingen (Polyommatus phlieas Lin.)}

Alis anticis infra fulvis, nigro-maculatis, margino oxtorioro canescento; posticis infra canoscentibus punctis minutissimis nigris. L. al. exp. $30-35$ millim.

Mas. et Femina: alis anticis supra fulvis nigro-macuJatis; posticis fuscis fascia marginali fulva, nigro-punctata.

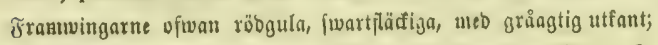
be bafre unbertill gråagtiga med ganffa fmå, fwarta punfter. ¿. c. wing $1 \frac{1}{4}-1 \frac{1}{10}$ t.

Szame od Syoun: iramuingarme ofwan röbgula ned fwarta flảcfar; be Gnfre bruna med ett röbgult, fwartpunftrrabt banb 1 utfanten.

Syn. Pap. phlazas Lin. F. Sv. 1078. Mäbn. Pap. t. 72. โ. 363. Text. 54. 38. Ilesperia Vabr. E. S. III. 1. 311. 178, Jyexna 0chs. Schm. I. 2. 87. IV. 27. ot 
148. Treitseh. \&. 1: 76. Zett, Ins. Lapp. 911. 4. Zephyrus lalm. Pap. Sv. 93. 9. Polyommatus God. Bt Dup. Lepid. 1. 204. II-S Texi. J. 134. Gerh. Lycwnon. t. 5. f. 4 .

2ar. b. Alis posticis supra uaculi coorulois ante fascian fulvaw marginalon.

Bafwingarne ofwan med Gli̊ fädar framom bet röbgula truetbanbet i utfauten.

Zephyrus Phlceas Ilalm. var. b. I. c. Lycuea Phlacas Zett. var, b. I. c. Pap. Phloeas lübu. I. c. f. 362.

Bar. c. Alis anticis supra fuscis, disco tantum fulvo, nigromaculato.

Frammingarme oftwan Gruma, blott $i$ biffen röbgula od,

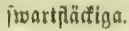

Pap. eleus Habr. E. S. Suppl. 430. 180-181? 0chs. Sclim. 1. 8. 90 ?

Stres(5)ulbwingen är ben alluănnafte arten inom flägtet o(t)

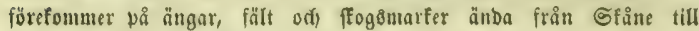

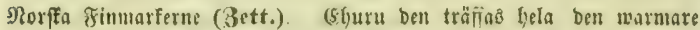
årstiben, är ben borf allmännaft ou mairen $i$ Mat od början af उัuni, faut jeban åter om böften i 2lugufti, få att ben bar egentligen twenne generationer om året.

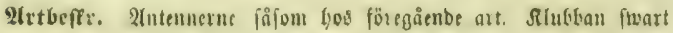

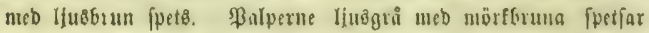
od) แnbertial meb Gucitagtign går. Dmfring ägonen en hwit

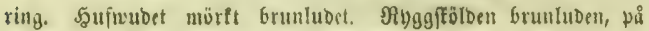
ffulbrorne od) bafofraßs gråagtig. 2afoomen ofwan fwart ned

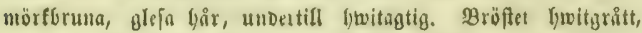
lubet. Jैötterne Gwitgrå ineb brunringlabe tarfer.

Samme oŕs bona. Framwingarme ofwan röbgula, gulb. glänjande, meb fwarta fläcfar, af lywilfa nuenue finmas i bift, fältet o(t) be B̈friga Gilba cn uregclbunden, föld twertrab nâr uare utfanten, lywilfen ăr frebt graibrun. SBafwingarne ofwan fwartbruna, glänjanbe, meb ett tenuligen brebt, röbgult twertanb

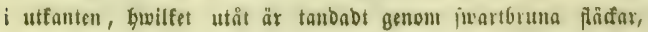


fom fammantlänga ucd ben fmalt brunfärgabe utfanten. Fram= swingarme unbertiff $i$ fpetfen och längb utfanten grå; för öfrigt röbgula meb famma fwarta tedfning fom ofwan, lifiväl meb unban. tag beraf att $i$ biffeältet finnas trenne fmarta fläđđar, lywilfa, lift alla be öfriga, äro fantabe med gult eller lywitt. Bafwingarne unbertill grå, mer efler minbre, färbeles mot utfanten, ftötanbe $i$

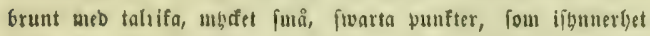

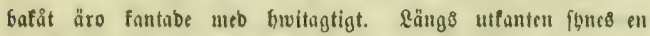
otgblig antybniug tifl öfre floand röbgula twerbano. Eranfarme ofwan Gwita, unbertill grå.

3ar. b. ffiller fif från füregåenbe enbaft berigenom, att innanfôr bet röbgula banbet på Gafningarnes öfre fitba finneß en rab temligen ftora, Glå đäưar. Jlas. ot Fum. Träffia till. famman med ben allmănna färggarieteten.

2ar. c. Gar framtwingarne på Jifre flban bruna od enbaft $i$ biffen röbgula; neen bet röbgula är ljär bnftrare, blanbabt meb brunt od be jwatta flädarue fürre, entbaft tybliga i biffert.

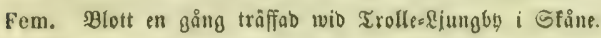

Earven fall wara ljubgrön mo en gul eflex bügräb linin

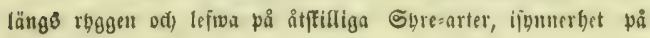
Rumex acetosa.

4. Ormmots-fuldavingen ('olyommatus helle W. V.) Alis infra fulvis, posticis griseo-infuscalis, omnibus fascia postica fulvo-rufa, serie adjaconte macularum Irigonarum nigrarum, intus albido-marginatarum, punctisque albo-cinctis plurimis, in disco sparsis. L. al. exp. $25-30$ millim.

MIas : alis supra fuscis, valdo violaceo nitentibus, punctis nigris minutis sparsis, prosertim in anticis.

Femina: alis anticis supra fusco-fulvis nigro coerulooquo maculatis, rarius violaceo inductis; posticis fuscis fascia marginali fulva, nigro punctata.

WBingarne unbertill röbgula, be Gafre ftötanbe i brungriatt, alla förfebba nảra utfanten meb ett röbgult twerballo och en berintil bes lägen rab af jwarta, trefantiga, init meb gruitt begränfabe fläđă, 
faut befutom i bifen fiera fpribob, juarta meb buitt omgifna pune. ter. \&. \&. wingfp. $1-1 \%$ t.

Samue. Wingarme ofwan bruns med fturft biolett glans od) fmå ipricba, jwarta punfter, îunnerbet på framtvingarne.

Sona. Frammingarne ofwan brunagtigl röbgula meb froarta

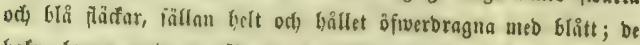
bafre bruı me elt röbgult, funartäăfigt band $i$ uttanten.

Syn. P. helle W. V. pr. 181, llesperia Vabr. E. S. III. 1. 312. 181. Papilio Hïbı. Pap. t. 67. f. 131-133.

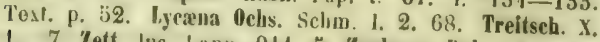
1. 7. Zett. lns. 1.арp. 911. 5. Zephyrus Dalm. Pap. Sv. 93. 10. Polyommatus Gool. of Bup. Lopid. II. 484. pl. 23. f. 5. 6. II-S Test. I. p. 130. Pap. amplidenmns Esp. schm. I. t. 58, c. 8. f. 4. p. 46. ot t. 63, c. 12. f. 5. Pap. hille Thbg, In. Sv. II. p. 40. Polyommatus Qerh. pl. 10. 1. 4.

Dennก wađta art är temligen allmän uti fpogåtrafterne på torrare ftăllen i Nort od) WBefferbotten unber Juni od Suli månaber. Sfunnerbet är ben talrif längs hela tuftlanbet från Sinbbect, i grän. fen emeflon $\mathfrak{B}_{\text {efter }}$ vil) Rorrfotten, äfwenion wio suleå elfivene

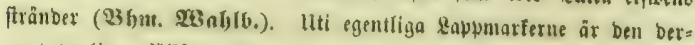
emot temligen jäLfont, men ftunes lifwäl ber od hwar på paşande Lotalex, fåjom nib Quiffort od, Degerfors (Bett.).

2Irtbefer. 2(ntennerne înarta meb byita lebafbelningar. Slubs Gan fwart, i buterfta fpetfen röbgulugtig. Bajperna broita, i fpetfen fwarı, unoertill fratft grålubna. D̈gonen fiwartoruna,

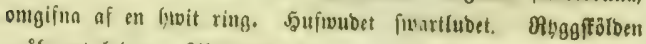

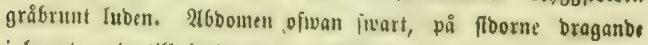
i brunt, unbertill bwitagtig. Brōftet liuzgerñtt, lubet. Fötterme Glwita med fwartringlibe tarier.

sanuc. WBingarne ofwan fwartbruma, $i$ bifen fwagt robs gulagtign, meb biolett gland, fom iitmuerbet är gaupe ftarf pฉ̊ De fräure; utfanterne och bafwingarnes fromfant iwarta. WBid bifffältels flut finnes en fwart ftrect, fom iffunerbet är grof ods tyblig på framtwingarme, Gwareft äfwen ftunes en rab fmärre

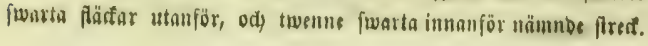


Bafwingurne bafwa $i$ analgörnet ett par röbgula, med fwart

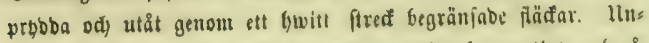
bertill äro be irämure röbgula ợ be bafre brunagtigt gulgrå, alla meb ett gentenfamt mörfare röbgult banto $i$ utfanten, bwil.

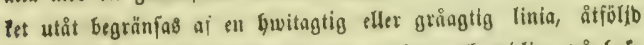
a) fwarta fired, fom ifpmerfyet äro grofwa ody ryblign jå baf= wingarne, od lywilfet inåt begränją af en rab trefantign fwarta flädar, af bmilfa hywarbera hafwa en luwit triangel, fom meb fpets fen wänber inåt wingen ợ på bafwingarue går längre inåt biffen, än på frammingarne. Deşia febrare bafwa särmaft mu beftrifna twerband en twerrab af fwarta punfter, twib bifffältete flut en od) 1 flelitio biffältet twenne famt nära infanten on amnan fwart punft eller fläc, alla omgifne lywarbera of en biwit ring. Bafwingarne Gajwa fpribon fwarta puntter $i$ biffell, men

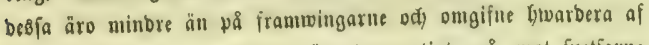
en gulagtig ring. Eranfarne äro brunagtigt gra, mot fpetiarme Grwitagtiga.

Syouau, fom lill wingarmes unbre fiba lifnat fannen, är på frammingarnes öfre flo robgul meb met eller minbre brunt wib wingarnes bas od ofwer berå ta. Sällan finnes fwag violett glans. Frammingarne lafwa uära utfanten twenne twer: raber of jwarta punfter, fom inat àtfolting af rlảagtiga; $i$ biff: fältet finnes en frrart punft ods wib twernerben en amnan. Batwingarme äro enfärgabt fwartbrunn meb ett röbgult tivere banl läng utfanten, grviffet är utåt förfebt med ell rab fwarta

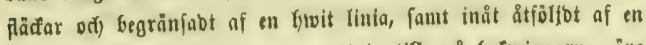
rab blå flảcfax eller punfter. Unbertil po̊ bafwingarne ảro merenbels be fwarta punfterme, närmaft bet röbgula twerbanbet, mera bilbanbe en twerrar, än fallet oftaft är hos bannen.

Sarven är luben, enfärgabt grön meb n:örf rGgglinta, gult

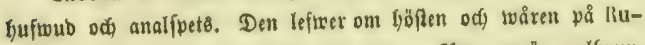
mex acotosa od Polygonum bistorta. \$uwau är gulficum, 廿å abbominalregionen bollagtig, meb fruarta långêtred oct) grofwa puntter. 
Sliggtet : Lycaena. (FABn.) Ocus. H-S.

(Polyommatus Lats. Boisd. Ind. Meth. Argus Boisd. Icon. ZerT. Zephyrus DaLM.)

Antemme longiores, clavatæ; clava brevior, comprossa 1. excavata, sat doterminata.

Palpi squamati, infra dense pilosi, apicilus subnutantibus, triarticulati. Articulus medius reliquis conjunctis Iongior; basalis minutus; ultimus basali longior, gracilis, latitudine fero conformis.

Ocull aut hirli, ant nudit.

Pedes ut in genere precedonte, sed articulus tarsorum primus tibiis semper gracilior et articulis reliquis conjunctis brovior; tertius secundo, hic tomen quarto longior.

Alæ rotundatx, integræ; posticæ in margine interiori haud oxcisid.

Costre alarum ut in genere præcedonte; anticarum 10, sed 6 ef 7 e locis diversis, illa sola ox angulo antico areolx, bæec vero in medio inter 6 of 8 oriuntur; ramuli coste septimx proportionaliter Iongines, quam in genero precedente.

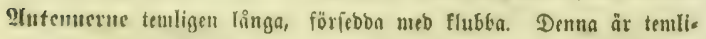
gen fort, fammantrydt eller urtyolfab och ni̊gorlunba beftämb. अalpertue fjälliga, unbertill tätt lubua, meb något lutande fpetfar, trelébabe: meblelfta leben längre än be biriga tillfamntantagua;

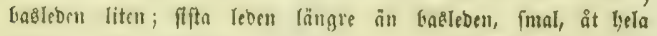
lẳgben nåftan lifformiģ.

Ögonen antingen fuona eller nafun.

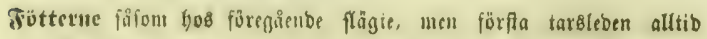
imalarc än tibierne od) fortare än be offriga leberne tifljamman= tagna: Den 3:bje leben lângre än ben 2:bra odı benna längre än ben 4:ber.

M3ingaruc runbabe, belbräbbabe; of liafre i inbre fanten föga

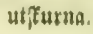


Rerverne po wingarme făjoin hos iöregåenbe flägte; framuingarnes nerser 10 , men 6 ợ 7 utgå från olifa ftällen af biffältet. Den förra enfam från befi främre börn, Den febuare beremot nuibt emeflan ben 6 ori) ben 8 ; Den 7:Des grenar ãro äiven

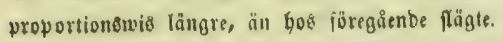

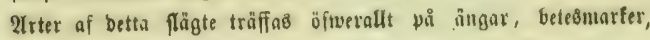

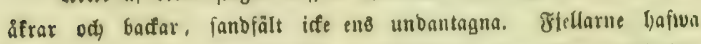
ăfwen flua arter, hwarai några uppifiga änba till frögränfen.

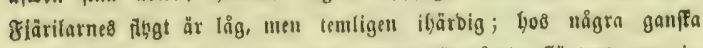
inabb. Deras larber lifna till cormen föregående flägtene, men be äro fortare än hoo Polyommatus od fulltigare famt minbre af=

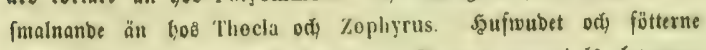
åro ganffa fmå, fuappaff iquliga. Siroppen merendels betwuxen

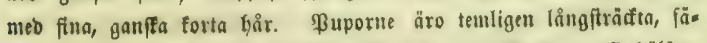
ftabe wio eı vextftängel, wanligen uära forben, flunbom ttll bälften Dolba $i$ benjauma.

\section{Tabula sublivisionum hujus generis :}

1. Alæ postice infra punctis nigris fasciaque maculari marginali fulva.

A. Oculi nudi : L. battus. L. argus. L. optilete. L. aquilo. $L$. agestis, $L$. eumedon. L. Donzelii.

B. Oculi hirti: L. alexis, L. adonis, L. dorylas. L. icarius. II. Alæ posticæ infra punclis nigris, absque fascia marginali fulva.

A. Oculi nudi: L. arion. L. alcon. $L$ cyllarus. L. pheretes. L. alsus.

B. Oculi hirti : L. acis, L. argiolus.

I. Bakvingarne undertill med svarta punkter och vid utkanten ett rödgult, af flickar bestiende tverband. A. Ögonen nukna.

1. Fetörts-Mlavingen (Lgctena battus W. V.)

Alis infra albidis maculis quadratis of rotundatis, magnis, 
sparsi ef seriatis nigri, ; po:licin fascia continua fulva, I. al. exp. $25-30$ millim.

Mas. : Alis supra niyris I. nigro-luscis, disco coeruloomicante, circulis cooru!eis in margine maculisque nigris in disco.

Femina : Nis supra nigris, ut in maro maculatis, disco vix cooruleo.

Wingarne unbertill bwitagtiga med qwabratiormiga eller run. Dabe, flora, fpribda och i raber ftällda fwarta fläđar; be bafre nteb ett fammanlyängartbe röbgult land. 2 . e. wingfp. $1-1 \frac{1}{4}$ t.

Sgante. WBingarme oftwall fwarta, eller fwaribruna nteb glänínonde Gli biffe, blå cirflar $i$ utfanten od fwarta fläđar $i$ biffer.

Soura. WBingarme of wan fwartagtiga, flä̆abe fåfom bos Lannen; $i$ bifen fnapt Glåagtiga.

Syn. Pap. ballus VI. V. p. 185. Ilesperia Vabr. E. S. III. A. 300. 137. Papilio Nübn. Рap. t. 66. f. $328-330$. Toxt. 51. 29. Lycrena Ochs. Schm. 1. 2. 64 . IV. 26. Gerh. pl. 22. T. 2. Zephyrus. Dalm. Pap. Sv. p. 400. 25. Polyonmatus Gol. ot Dup. Lopid. II. 195. pl. 25. f. 7.8. Ilesp. sedi Nabr. E. S. III. 1. 286. 98. Pap. telephie Esp. Schm. 1. 1. 41. c. 17, f. 2. t. 94. c. 49, f. 5.

Deuna jällynta art är inom Sfanbinabien bittills enbaft träffab

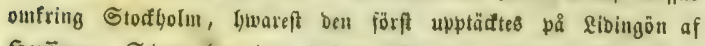

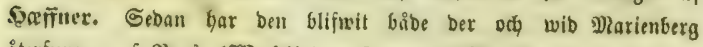
àterfunnen af \$rof. (:33ab16.). Den uppebållex fig om waren tå ody onfring be flippor, ber Sodum tolephium, Iarvene egentliga näringsplanta, twexer.

Artbeffr. 2Utennerne fwartbruna meb hwita ringas. Slubban fwart, i fpetien blåagtig. ßalperne blågrå, stterfla leben fivart, unbertill lubna meb fwartigtiga ficfelbâr. N9Qggftỏben brungrå, laben. 26boomen ofwan fwart med gleía brunagtiga bår; un= bertill hroitagtig. SBrōftet blågrått, lubet, Fỏtterne blågrå meb brunxinglabe tarier. 
Syatui. Waingarne of wan funata eller furatbruna med jwarta, mex eller minbre tholiga plädnx, if̈bmertyet på be

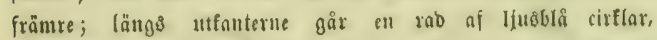

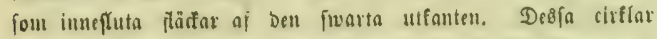
åro minbre thbliga på framusingarne, än på Gaftvingarne.

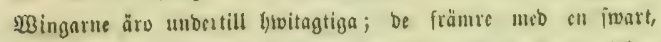

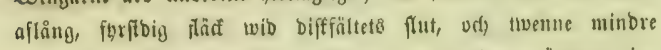
innanför benua, ben cha $i$ bifffältet, ben untora närmare in=

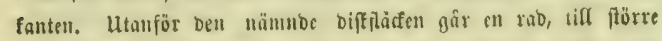

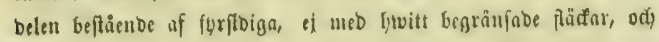
längs utfanten twenme raber anbra bylifa, be gttre lifwäl run. babe. Bafivingarne lyafwa twas till tre runbabe juarta fläffar eller puntter wio bajen, en aflång, ibrfibig twio biffältet? flut, on ftarft Gågböfo rab bglifa twert öfwer wingen vds twemne rae Der anbra, rumbabe, längo utfanten. (Emellan be båon iffnüsunta finnes ett fammunbäuganbe röbgult twettanto. Franfarme äro hucita med förre fuartbruna fläfar wio wingnerverneo utlopy.

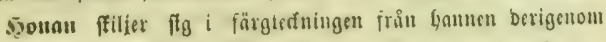
att wingarmes ọire floa är mera fwart, flwarföre be fwarta

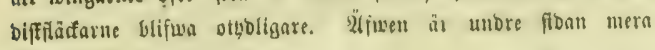
fötanbe $i$ brunt.

Encuen feftrifures tunra flögrön, fluludell, meb en mört,

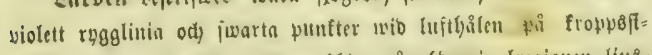

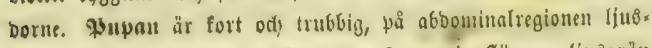

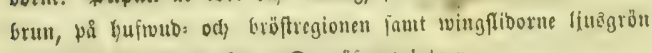
meb blefa, Gruna punfter. Den öjwerivintrat.

\section{Allmämua Brívingen (Lyciena argus lin.)}

Alis infra cinurois I, canis punetis ocellarihus sparcis ot soriatis nigris fasciaque nigro maculata, in posticis argentoocoeruloo ocellata, fulva, adjaconle lascia continua alba; clava antonnarus supra nigro, infra fulvil, L. al. oxp. 20-27 millim.

Mas. alis supra cooruleis margine lato nigro, fimbria alla.

Femina: alis supra fuscis, ad basin coeruloo-pulvoru- 
Iontibus, in disco plus minus coeruloscontibus, fascia intramarginali maculari fulva, interdum deficiente; fimbria plus minus infuscata.

2Bingnrme unbertill affegrå efler iฮ̊grå med fpribba oḍ $i$ raber

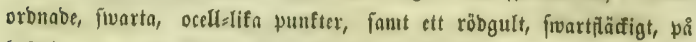
bnftwingarme uted flfwergläıfanbe blå ocellpunfter förfebt banb, intill fivvilfet fiunes ett annat fommantyänganbe, of bwit färg; antennfluba

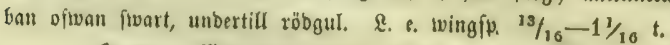

Sanuc. WBingarme ofwan mörfGlå med breb fwart utfant od) brita franfar.

Syoua. Wingarne ofwar bruna, wio Gafen blåłubrabe, i biffell mer elles minbre blågtiga meb ett robgult, flunbom föriwinnanbe fäcfonnd $i$ utfanten ợ neer eller minbre bruns agtiga franjar.

Syn. Pap, argus Lin. F. Sv. 283. 1074. Kephyrus Valm. Pap. Sv. 100. 26 Pap. idas Lin. F. S. 284.1075. Argus Zeti. Ins. Lapp. 913. 8. Lyeæena agon Ochs. Schm. I. 2. 57. Pap. agon llïbu. 1'ap. ใ. 64. ศ. 313-315. Lycena agon Gerb. pl. 23. 1. 2. L. leodorns Gerh. pl. 23 f. 3.

Demna art äน allmăn ofwer hela Gfanbinatien från bet fubliga Gfinte till bet norbliga Tromea. Dorf är ben $i$ àtftilliga mellan. liggarbe prosinfer mera fälli̧̧nt, fafom i D̈ftergötbland (3tcub.) Den fürebrager betesmarf meb enefuffar od ljumg, faunt ängsbadfar

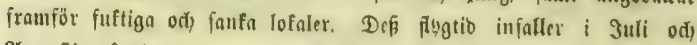
đugufti månaber.

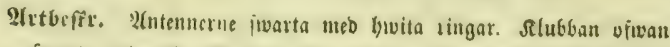
fwart, unbertill till bela fitu längb röbgulagtig; ofwan mot jwetjen förfebo med en huvit twerfäđ. \$alperne blåagtigt hivita, unbertill lubna of bisita od fwartagtiga bår; ytterftu leben iwart. De nafna ögonen jwarta, med frun, ni̊got glänjanbe

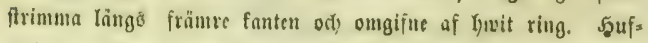
wubet encclan ögonen fwartlubet meb en långe̊gåenbe blå frimuna

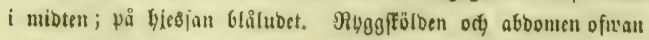
blâtubun; ben ironare undertill buitluben od butepubrio. \$an. 
nenв analborfte krungrå, mot rygen mörface. BBröftet lubet, blålywitt. Fötterme blåagtigt kwita meb jwartriinglabe tarjer.

Sqnume. Wingarme ofwan mürfbla meb breb, fwart utfant,

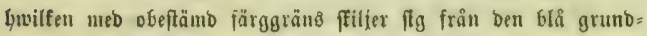
färgen od̆ yå bafwingarne flumbom aitfölices of en rab fmarta punfter. Framwingarneह främre fant fmalt hivit, unbertill

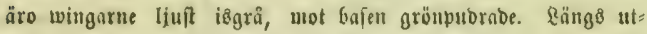
fanterne gå ett röbgult banb, jom inåt begränjag af en rab f)alfmånformiga, fmarta flăfar od) utât bay en rab mot besfa fwaranbe punter, bwilfa po̊ irammingarne äto fwarta, men pâ

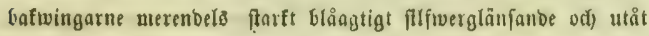
begränfabe af en Gwitagtig lalicirfel. Bå be främre är bet röbgula lianbet otybligare ody mattare, än bå be bufre. \$3a alla mingarne åtfölles bet of ett fywitt Ganb, fom ftunbom, bå grumb.

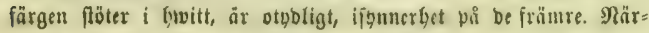
maft betta går ofleer wingarme en rab fwarta punfter, fwarbera

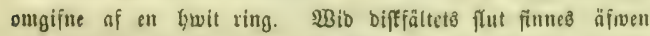
en fwart meb lywitt omgifwen fläđ odj immanför benuta få baf.

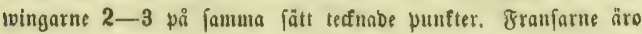
blvita od) åtföltas unbertill of en fin, fwart linia.

Scouau har wingarnes unbra fiba aftegaá, fötanbe i brunt,

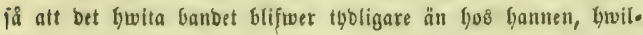
fen ben eljeft gaăr lifnar. Till wingarnes öfre fton varierar Gon näftan $i$ vänblighet. Wingarne äro ber bruna, wib bafen blåpubrabe; för ôfrigt antingen enfärgnbe, eller of $i$ biffen mer cller minore blågtiga, flunbom i utfauten prboon med en rab röbgula fläđar antingen på båba wingpareı eller blott på be bafre. Eranfarme äro mer eller minore brunagtigt grå, neren= Dels endaft gwwita $i$ frammingarnes fpetfas.

garven lefwer pi̊ Melifolus, Sarothamnus, Trifolium, Thymus od Âtera allora wetter.

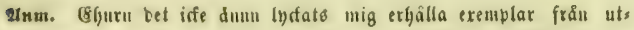
lanbet af Forfattarues I,ycana agon, nufer fag boof beuma för ibentiff med stimes $\mathrm{L}$. argus, emedan afla be afbitbningar, fon warit mig till: 


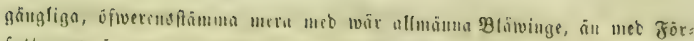
fattarues $L$. aryus, fowilfen befufem iffe mig veterligeu äun àr funneu

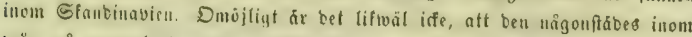

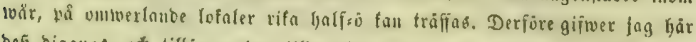
Def biagnos, od tillagger ben tills wibare bet uamu Boisouval bilbabt:

\section{Lycana calliopis Iloisd. *)}

Alis infra canescenti-cinereis punelis ocellarihus sparsis et seriatis nipris, fasciaque niggrn-maculnta, in posticis aryenten-coeculeo ocellata, fulra, maculis sagitlatis, plus minus confluentibus, alhidis adjectis; clapa antennarum nigra apice fulro.

Mas, Alis supra coeruleis, margine angusto nigio.

Femina. Ahs supra fuscis, disco coerulescente, fasciaque intramarginali fulra maculari.

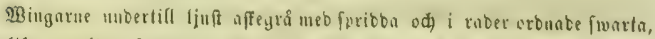
vcell:lifa bunfter faut elt rögult, fmatthangigt, bi bofmiagnene meb filf: werglanfanbe, bia scellsunfter forfert tmerbano, intill fowilfet fiunas pil= formiga, mer eller minbre fammanflytanbe flảdar; antenuflubbau fwart meb röbgul fuete.

Saute. SBingarme ofwan mörfola meb fmal, fuart utfant.

sona. Dimgarme ofwan bruna med blangtig bift odj ett röbgult fikd banb i utfanten.

Sjo. Angus calliopis Butsn. Icon. p. 52. P.x, aryus Haß.. Pap. 1. 64. f. 3:6-348. Text 50. 23. Lickes argus Ocas. Sclim. 1. 2. 62. IV. 26. \$146. Treitsci $X, 1$. 71. UI. S. 1. b2, f. 247 Genn. 1. 24. f. 1. Polyomantus. God, el Dup, Lepid. I. 218, pl. 11. f. 1. pl. 11. tert. f. 4

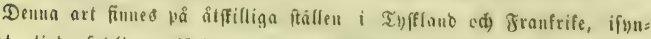
nerfjet uti be fubligare läuberue. Snom Daumat frall ben ej forefomma.

\section{Violettfirgade Gỉaviugen (I.jexna optiluto Esp.)}

Alis infra cincreis punclis ocellitribus nigris sparsis ot seriatis, lunulistuo acellalis duabus lulvis ad angulum ani posticarum. L. al. oxp. $30-35$ inillim.

") Specinioa suecana hujus speciei uon vidi, quare etiam nomen a Boisduval impocilum: a! libui. Zephyrus arzn: Drhat, in Suecia rulgalissimus, est L. ayoni maxime attinis, ut e figutis ritalis apturel. Specimina exotica von vidi 
Mas. Alis supra obscure violaceis marghine nigricante tonuiero.

Femina. Alis supra fuscis disco plus minus roorulosconte.

Wingarne unbertill affegrå meb ocell:lifa, fwarta, fpribba od $\mid$ raber orbnabe punfter, fant twenne oscli=lifa röbgula fläar $I$ bats wingarnes analfgörn. $\ell$. e. mingip. $1 \frac{1}{4}-1 \frac{1}{16}$ t.

Senume. פBingarne ofman mörft violettbla med fmal, fwart utfant.

Şuna. Wingarme ofwan bruna, i bifen mer eller minbre blåagtiga.

Syn. Pap. optilete Esp. Sclim. 1. t. 79. f. 45. p. 136. Ilesperia Pabr. I. S. III. 1. 297. 131. Papilio Hïlan. Pap. t. 63. I. 310-312. Text. 30. 25 lyeæena Ochs. Schm. I. 2. 51. IV. 26 \& 146. Zephyrus Dalm. Pap. Sv. 100. 24.- Argas Zett. In- Lapp. 9/3. 7. Polyoumatus foud. of Dup. Lepid. I. p. 198. pl. 26. 1. 3-4. Aierh. 1. 16. f. 4.

2̌f fwen benna Blåminge träffos öfwer nllt på Sfanbinabien uti

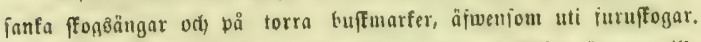

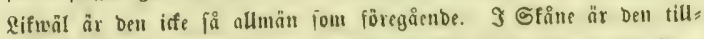
odjme temligen fällibnt. Den går lifa lößgt mot norben fom füres

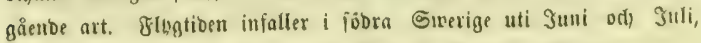
i norra od i Norige beremot uti కuli od 2tuguft mannober.

2trtbeftr. 2(ntennerne fwarta med linita ringar. Slubbun jwart, med en lywit fiäd ofwan mot fpetien. Balperne grâ meo fluart fpels, undertill fmartlubna D̈gonen jwarta, omgíma of ell fyvit ring. Şuimubet emellan ögonen fwartlubet ucb ent längos

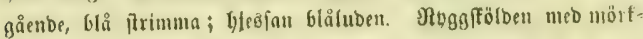

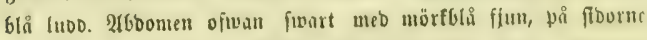
Glâgrå, unbertill Ģwit. Bröfftet Glåagtigt lywitt, lubet. Fötterne Glwita med bruna, ofwan grwitiläcfabe tarier.

5anuse. Wingarne ofwan mörft violetffärgabe meb inbigos glans ; $i$ utfanterne imalt fwarta. WBingarne unbertil afegri̊ ured en afläng frortagtig, meb lywitt omgifwen plär wio biffe 
fältetê flut; utanför Denra en rab luwarta med buvitt omgifna punfer od derefter i utfanten twenne raber, Iifaleber med lywitt omgifna, fläđar, of btwilfo ben inore på framtuingarne är tyoligate ån ben yttre, men på Gafwingarne båda lifa tyb=

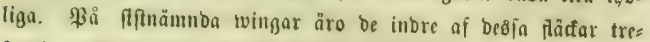
fantiga ody be sttre, bem motiwaranbe, runbabe. Närmaft analo

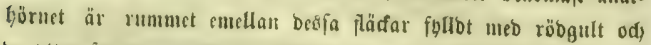
be buttre fwarta punfterne blipubrabe, fa att tivenue (fitunbout enbera otyblig) oceller berigenom bildas. Rärmaft franjarne ï̈s per en fmal fwart linis. Franfarme äro $i$ fpetfarme Gyvita, men wib roten brunagtiga.

Sุunan, fom till tvingarnes unbre fiba lifnar fyannc॥, ât ofivan fwartbrun, endaft blångtig antingen äfwen $i$ bifecl, eller of flott urio bafen. Defítom bar Gonan ofwan flunbom en orangefärgab fẳ̆ wio Gafivingarnes analljōrn.

\section{Mignordiska IBlâviugen (Lycæna aquilo Boist.)}

Alis infra cinereis basi coorulescente, anticis macula discoidali nigra, albo marginata, serio punctorum ocollatorum, altoraqu1) macularum nigrorum albo-marginatorum intramaryinali; posticis maculis difformibus albis, plus minus confuontibus, fasciaquo intramarginali fulvosconte obsoleta. L. al. exp. 27 millim.

Femina. Alis supra cinereo-subcoeruloscontibus macula discoidali nigra, albomarginata; anticis sorio duplici punclorum albidorum.

Wingarne unbertial affegrå, twio bafen bläagtiga; be frảmre uneb en jwart, bwittantab fläa i biffen, en rab fiwarta, ocellslifin punf= ter od) en annan af fwarta, husitfantabe păafar i utfanten; be bafre meb olifftora, neer cller mindre jammanitbtande, blwita iärfar ody $i$ utfanten eft olgbligt, röbgulagtigt banb.

Soun. B3ingarme ofwan blågatigt affegrå med en jwart,

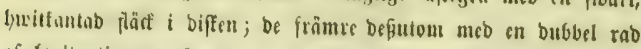
af blwitagtiga puntter. 
Syn. Argus aquilo lloistl. Icon, p. 62. pl. 12. ศ. 7. 8.

Polyommatis fiod. of Daps. Leppicl. Suppl. I. p. 295. pl. 47. f. 6. 7. II-S. tah. 71. I. 343. 344. tierh. pl. 19. f. 1. (figura mala). Polyomm. Frankilıni lions Appendix. pag. 69 pl. 1. a. I. 8.9 .

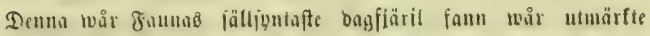

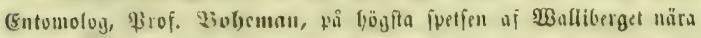

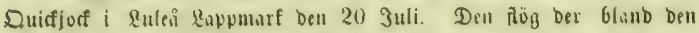

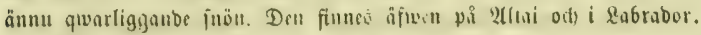

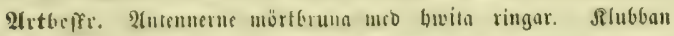
mörfbrun med en quvitagtig flä̊f oiwan nära ipetín. Đalperme

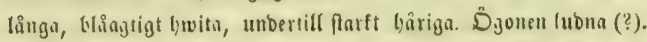

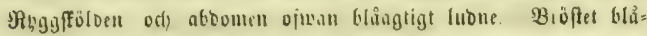
grålt, lubet. Fötterte - - -

Şouล. W3ingarne ofwals blåagtigt gri̊ med ell fiwart,

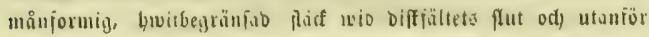

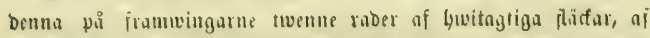
lyvilfa ben bttre, fom går nära utfanten, àr othblig, nîftan omảrtig. I utfanten of bafisingatme finte ell rab fwarta, med fuwist omgipna punfter. Wingarne unbertill offegri, wib bajen blåagtiga; be frïmre liujac, wio bifffätets flu jürjeboa

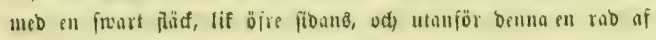
5-6 fwarta, med fowitt ougiena punfter, af builfa be two utfantell of januma wingar går (1! amman iud af mera tres

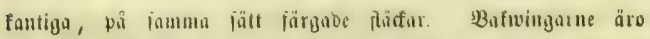

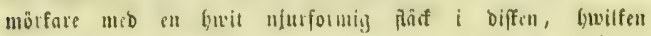

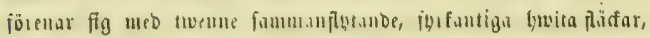
fommanbe frin mioten af framfanten. Snnauiör mibtelpläfen funns 2-3 busita minore punfter, ody utanför jomuna plä̊f

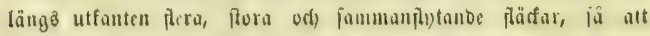

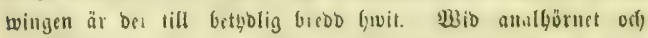

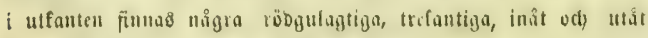

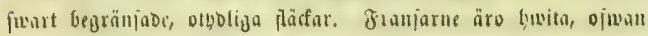
od) unbertifl aitiöliba of en fin, fivart linia. 
Syanuen, jom lag el fitt, frall lifun yonan, men

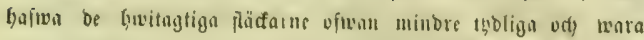
แtera blå*).

\section{Moidfäickiga Baivingen ( $L_{\text {, }}$ cena agestis W. Y.)}

Alis infri cunescontibus lascia matulari intramarginali nigro-maculata fulva, seriecur adjacente punclorum ocellatorum nigroram in posticis antico interupta; posticis macula modia

-) Desc. Antenna fuscæ anmulis olbis. Clava fusca, supra macula apicali albila. Palpi sat lowii, coerulec-albidi, infra dense pilosi. Oculi hirti (?). Thorax el abdomen supra cocrulesernte hisli. Perlue coeruleocinereu'n, hittum. Pedes - - - -

Femina. Nim supra cioereo-subcocrulescentes macula lunata, albomarginata, oigra in diseo, serieque in anticis duplici marula um albidarum, altera in medio inter cellulan et marginem, altera obsoleta in margine ipso. Als posteriores serie intranaginali punctorum nigrorum, albo-marginalorum. Ala infra cinerem, ad basin cocrulescubs; anteriores macula discoidali ut in parte superiori, sericque punctosun $3-6$, nigranum, albomarginatorum; inter ha ?, marisini interiori proxima, sunt foras locata. Series altera macularum trigonarum nigrarum, ejukmodi albo-einetarum, ad marginem cxterioren adest. Alu posteriores sunt in pate iuferioriobscurinres macula raniformi discridali allo, cum 2 quadrangularibus ef inter se coufluentibus, e medio marhinis ans rioris egresliontibus, conjunets. Intra maculan hane discridalem $2-3$ puncta alba atsunt, et extra uraculam eanden maculw majores el confluentes, plurima, in maghine exteriori sunt collocate, ut m.rgo lota sit albus. Ad angulum ani of ad margincm exteriorem macula-nonufla fulsw, triangulace, nigroterminata, sed obsoletiores, repeniuntur. Fimbrio albe, supra el infra al hasiu linea nigra tenuissima terminatæ.

Mas, quem non vidi, fenine similis describitur, sed maculas allos in colore magis coctulescente pagino superioris ularum antiearum minus distinctas gerere dicitur.

Speciem hane lepilopteresum nostratum rarissimam l.eleberrimus nnster lintomologus P'rol. Boheman die 20 Julii in carcumine Waili montis prope Quiskjock Lapponim Lulensis lepit. Spucimen, a nobis visum et descriptum, sat lasum fun, ut ocuion "l pedes discroliere non poluerimus. 


\section{4}

marginali alba, cum macula discoidali, cordiformi, non cohærente, et punctis ocellaribus, basi tamen anticarum impunctata. L. al. exp. 30-35 millim.

Mas. Alis supra fuscis maculis interdum fulvis marginalibus obsolotis, lineolaque nigra discoidali in anticis.

Femina. Alis supra obscuro fuscis maculis fulvis marginalibus, lineolaque nigra discoidali obsoleta in anticis.

WBingarne unbertill isిgrångtiga meb ett fwartiläftigt, röbgult pläđbanb $i$ utfanten, od) en berintill grânfanbe rab af fwarta, ocelle lifa punfter, bwilfen på Gafwingarne är framtil afbruten; be bafre $i$ midten af utfanten med en Lyvit flät, jom ef fammanţänger mé ben Lurita, biertformiga biffläder, fant fòriebba med ocellormiga punf= ter ; Framwingarnes bas utan punfter. \&. e. wing $\left\{\begin{array}{l}1 / 4 \\ 4\end{array}-1 \frac{1}{16}\right.$ t.

Saaure. Wingarne ofuan brunn, ftumbou med röbgula otgoliga fläforar i utfanten, fant meb en fort, jwart linia $i$ be främrę biff.

Souล. Wingarne ofwan mörfbruna med röbgula päffar $i$ utfanten, ody en otbblig, fort, fwart linia $i$ oe främres biff.

Syn. Pap, agestıs If. V. p. 184. Ilïbn. P’ap. I. 62. f. 303-306. Toxt. 49. 20. Lycrena Ochs. Schim. I. I. 44. IV. 26. Treitseh. X. 1. 70. Zephlyris Dalm. P'ap. Sv. p. 99. 23. Polyommatus (iod. of Bup. Lopid. I. 2 |3. p). 10. ᄃ. 4. pl. 11. tert. f. 3. (fig. incorrocta) Pap. medon Esp. Schın. 1. t. 32. suppl. 8. 1. 1. p. 330 . Lycena agestis Gerh, pl. 26. f. 1.

Bar. b. Alis supra fuscis, immaculatis.

Wingarne ofwan bruna, ofläcabe.

Ftärilen förefommer ganfta fparjumt på fanfa ängar ber od)

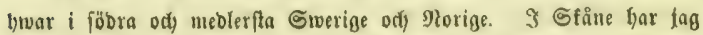
träffat ben wib Trolle Riungby od $\mathfrak{2}$. 23 ramb, i Blefinge wib Söl.

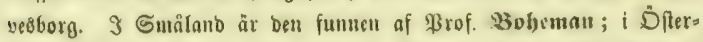

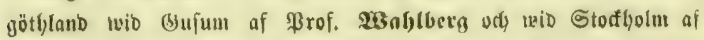
Denjanme. Det norbligafte ftälle inom Stwerige, Der wi weta ben wars funuen är Norberg, lyareft ben förefoumer någon gång uti ben fåfallabe Mutuftäften (Frebriah).). Jnom Norige är ben an- 


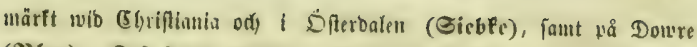

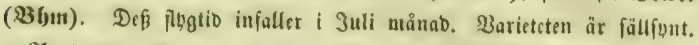

2rtbeffr. 2Intennerne fiwarta med bwita riıgar. Rlúsban frart,

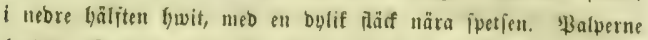
lurita, ofwan od) i ipetfen fwarta, umbertill latona med fwarta fticfelbår. D̈gonen fwarta med en jual, bı uแ, nâgot glänfande ftrimua frumtill, och outgifna meb en byit ring. Sgufwudet

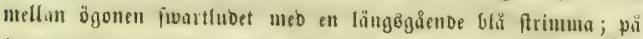

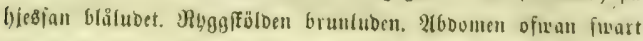

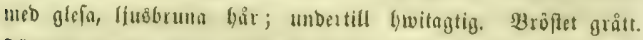
fötterne byvita mcD unbertill bruna, ojwan frunthäfiga tarjer.

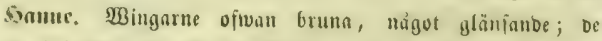
främre, $i$ iteliwa frantanten butita, med ett fwart fireff wio

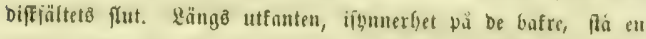
rab af trefontiga cleer lyalfuninjormiga, röbgula fläcfar. Lander. till äro wingarme isgråagtiga me ett butto af röbgula faifar, foul på båda wingharen äto lifa tobliga od̉ inât begränjade af fivarta of) bwita balfmånar fant utåt ătröljoa aj cll rab jwarta Hunfter pả Gavit botten. Märmaft innanfür betta band går en twerrad fwarta, med h)witt omgiina punfter, af liwilfin bå jrame

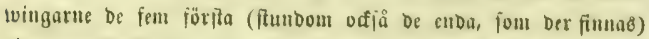
bilda en Parft Gojid bage med den fonvera fiban utätwänd, ớ be tsuenue (ftunbom blott ell, ftunbom inga) till infanten uärs mafte ftå niggot närmare utfanten, fi̊ at raben är ajbruten.

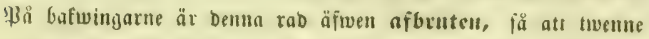
punfter fẩ nära hwaranbra od) iramtanten, längre ffildo frůn

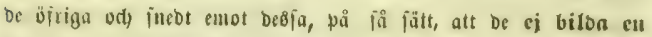

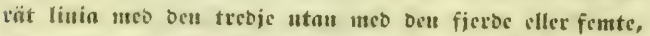

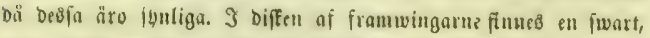
med hovitt omgifwen färf eller ftred, ods innanför benta fumas inga imarta punfer. 3 biffen af Gafuingarne fünes on bwit,

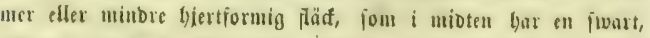

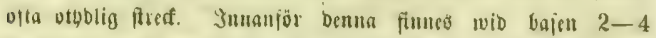
jwarta punfter, litnande be ojtiga. Frän miden af bet röbgula 
banbet $i$ utfanten gå en blwit fling långt framom tuverrabens 4:be ods 5:te punfter, af hivilfa flumbom enbera, funbom baiba

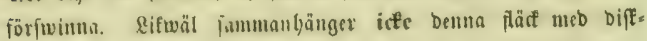
fiñđen, clyuru bell går in emot od flunbom nära intill bens famma. Franfarme äro biwita od) nârmaft Dem löper unbertill en fmal, fwartagtig linia.

Soutau Kifmar yaumen, men bat grunbfärgen ofnum mör= fare, ory fillebrs frammingarnes jwarts bifferted minbre thbligt.

23ar. b. Limas $i$ allt jörut beftrima, med unbantag beraf,

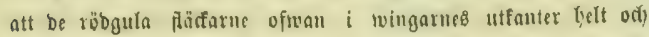
bållet fafnas, Mas. Fem,

\section{(6. IBrunagtiga Lblavimgen (I ciena eumodon Fsp.)}

Alis infra cinereic, basi vireseentibus, fascia maculari intramarginali, nigro-maculata, fulva, prasortim in posticis distincta, serieque adjacente punctorum ocellatorum nigrorum continua; posticis macula media marginali alba, cum macula discoidali striga ejusdem coloris coherente, et punctic ocellaribus; basi anticarum impunctata. L. al. exp. 28-32 millim.

Mas, ot Femina. Alis supra nigro-fu-ris lineola discoidali nigra.

WBingarme unbertill affegrå, wid bafen grönagtiga, med ett fwart=

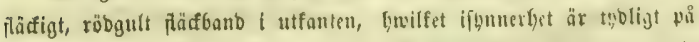
Gafuingarne, faut berintill en fanmant,änganbe rab af furarta, ocels lifa punfser; be Gafre $i$ atiben ai utfanten med en Givit fläcf, jom

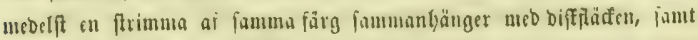
föricoba meb ocell:lifa punfter; framuingarme wib bajen utan funfs ter. \&. e. wingip. $1 \frac{1 / 8}{8}-1 \frac{5}{16}$ t.

Sanute odf houn. WBingarne ofmur fwattoruta med ent fwart fired i biffen.

Sjn. Pap. cumedon Esp. Sichm. I. t. 52. cont. 2. r. 2. 3. p. 16. Un̈lu. Pap. 1. 62. 1. 30 I. 302. I. 138. i. 70 4. 702. Text, 49.21. Jyerna Delis. Schm. I. 2. 48. IV. 26. \& 1i6. Zephyrus Dalm. I'ap. St. 99. 23. Polyommatus fiod. of IIIj. Lepid. II. 192. pl. 25. f. 1. 2. 
Airlı. pl, 25. f. 2. Pap. chirom Horkh. Schm. I. 16 b. (c) 280,16 .

3ar. b. Alis supra maculis intramarginalibus fulvi:

WBingaune ofwan med röbgufa Påfar i utfauterne.

Denta flärit är ci eller blanb de nllmảnnare. Dof thades ban förefoumma oftarc äแ jöregåenbe art, lywilfen ban ganffa umbet lifs

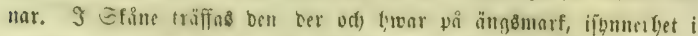
närlyten af volade ftällen. 9 D̈plergötly and är ben aflutîn omfring

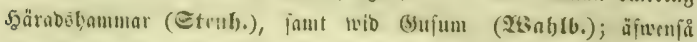

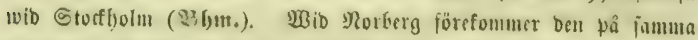
fälle fom föregånde nit. Snom Norige äl bon aumárft wid chrie

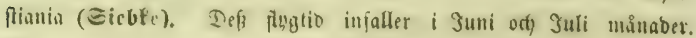
Barietcten är fäLfont.

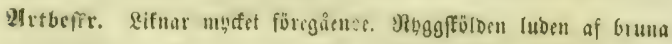
ợ 6låagtiga bår. Brỏptet blågråt.

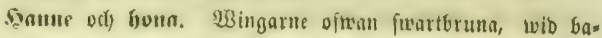
fen obetboligt sliagtign; be främure med en imurt feref $i$ biffen. Unbertill äro ringarne mỏrft affegrå, frötanbe i brunt, wib bafen grönagtign, ifgumerbet oc bafre. Desia lonfwa lings uts fanten ett basto of rürgula, inait farpt tillipetínoe ods ber med

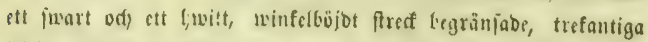

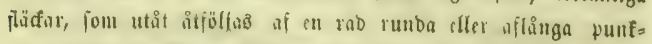
ter, utanför Gwilfa grumbfärgen är brvit. Eramuingarme bnfwa

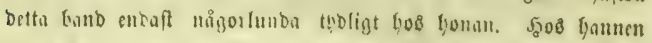

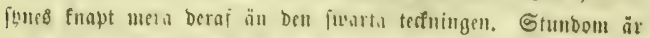

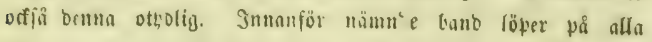
wingarne en twerrab af furats, med fewitt omgiina punfter.

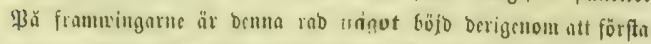
punften (från flumfanten) flit lämpıe inåt än ben anbra. Stun= Dom fafna ben, bả raben är allocics rät od) beftar endaft af

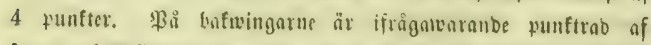

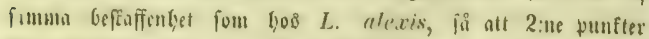

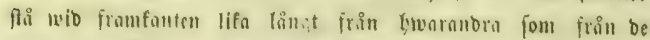

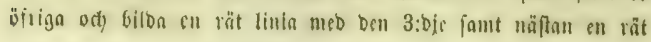


winfel mot be öfrige, Glvilfa aiter $i$ en tät linia meb bwaranora löpa till infouten, od) ef flingrande făfou yob L. ayestis od alexis. 7:be od) 8:be af besia pumfter fafnas flunbout. 3 biffen af liwarbera wingen finnes en fwart ftred,

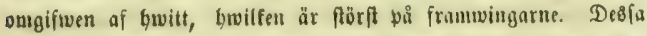
bafiva inuaniör nänmbe flrest inga punfter, men be bafre bafiwa wio framtanten cubafit $\mathrm{es}$, lifnanbe be ofriga; bucf är ben ftundom otydig ody fafnas äfwen bos ett cller annat exemplar.

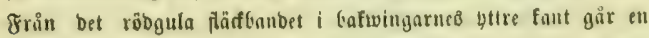

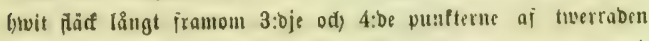

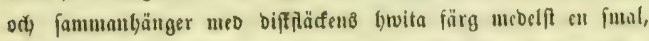

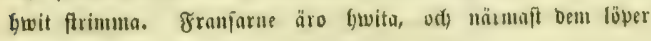
på wingarmes unbre fiba en jual, brun linia.

23ar. 6. lifnar allofleğ föregúende, men far pii öfre fibar

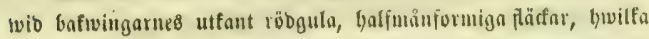
funboum blott äro antyboa wib amalgürnet, men bercuot plus= bour äro tubliga länge lyela utfanten.

\section{ซ. Donzels-\#Bávin to (Lycæua Donzolii Iboist.)}

Alis infra cinerois, basi viroscentibus, sorie continua punctorum ocellatorum nigrortum, macula di.coidali nigra albo-marginata, fasciacue marginali maculata fulva valdo obsolota; pesticis vitta media longitudinali alba; basi anticarum impunctata. L. al. exp. $2 \xi-30$ millim.

Mas, Alis supra viresconti-enorulois, margino lato lunulaque media anticarum nigro-luscis.

Femina. Alis supra fuscis, ad basin vix coorulesconsibus.

Wingarne unbentill affegrä, wio bajen grönagtiga, ureb ell lauls

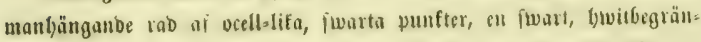

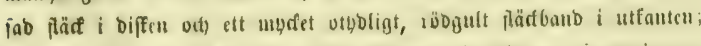

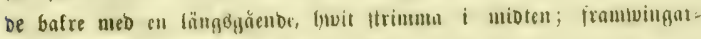

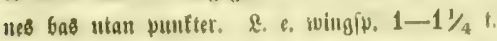


Saaune. 23ingarne ofman grönagtigt bli meb breb fwart. brut utfant ody en färof af famma färg $i$ mibten af frammin= garne.

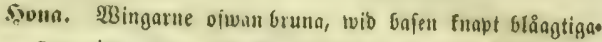
Sfrn. Argus Donzelii IBoist. Icon. 64. pl. 15. 1. 1-3. Yett. Ins. Lapp. 914. 9. Ilïhn. Рар. 1. 193. 1. 955-957. lycreua. Treitsph. Schm. X. 1. 64 . II-S. t. 73. f. 351 . 352. Ferh. pl. 19. ґ. 2. Polyommatus. God. of Dup. lepid. Suppl. I. 48. pl. 8. f. $1-3$.

görtienften af att bafwa wifat, bet benna fällignta s8låminge äfwen är en Gfmbinasiff art, tilltör Brof. Boheman, fon under fin refa till Norige 1836 upptädte Denfamma i Dalarne emellan Tranftrand od) 9) Rafung. Geban bar Den äfrwen Glipwit träffab of

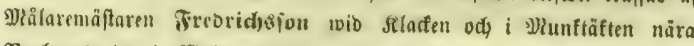

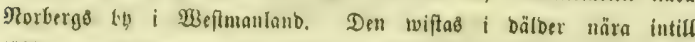
löffog ody fluger i Tuli. Ltom Gfanbinawien är ben funnen : Eranfrife.

Artbeff:. 2utennerne pwarta, Gwitringlabe. Selubban fwart, nebtill röbgulagtig, med hwit fpers ofwan. \$alperne houita meb fwart fpets, แnbertill meb fwarta od, Grunagtiga hår.

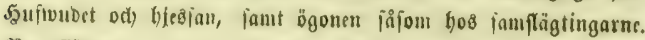
Itygeftöben luben af blå bår. 2t6bomen ofwan fwart, gleft Glåluben; unbertill bwit. Bröftet Gwitt, lubet. Fötterne brvita, me bruntădăiga tarier.

5anute. BBingarme ofwan größragtigt blå meb breba fwart:

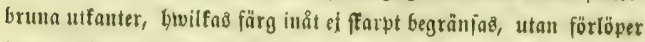
i grunbfärgen. Framfanten på framuingarme fmalare, på baf: wingarne brebare fiwartagtigt brum; på be forra $i$ utterfta fant

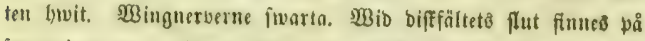
frambingarne sll fiwart flärf. Llıbertill äro wingarne affegrå meb onbliga, röbgula, trefantign flŏfar lăng utfanten, hwiffa

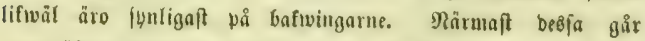
twert öfwer bwatbera wingen an jammanlyüngande rab af jwarta, med hwitt omgifma punfter, fowilfa på frammingarme äro nogot

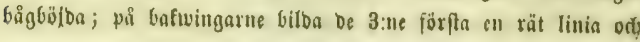


winfel mot be öfriga, fom närmare nualbörnet göra en liten

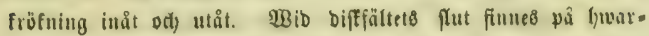
Dern twingen en fort fmart ftreff, lifaledes omgifwen af tywitt. 3uแanför benna finues yå framivingarne ingen qunft, men på Gnfwingarne flunbom en otyblig nära framfanten. Nhära bufen

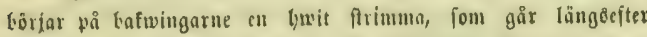

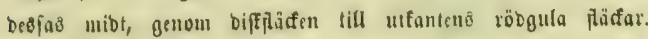
Jranfarme äro lywita ody Gegränfas unbertifl of cn butu, fin linia, lywilfen femite fig lyar en anman, of forta ftreof beftis elloe, otbolig od lywit.

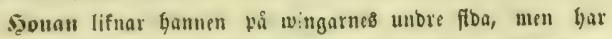
be röbguโa flätarne något tholigare, od) är ofwan brun meb blingtig anftrbfning wib wingbajen, famt liar funbom $i$ utfans

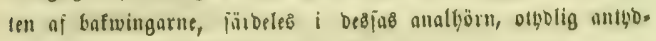
ning till röbgula त̈äcar.

\section{B. Ögonen ludnu.}

\section{Puktörne-Rblâvingen (Lycæna alexis W. V.)}

Alis infra aut cinerascentibus ant griso:centibus la-cia marginali maculari nigro punctata of lineis nigris, accute angulatis intus terminata, lulva, macula posticarum ante bujus medium alba, ad seriom continuam punctorum ocellatorum adscendente, maculaque discoidali nigra, albomarginata ot punctis basalibus ocellatis; anticis punctis nigris, otiam ocellatis; fimbria omnium alba, L. al, oxp. $28-32$ millim.

Has. Alis supra subviolaceo-coeruleis linea tenuissima marginali nigra.

Femina. Alis supra luscis, disco plus minus cooruloo, sepe ocellis intramarginalibus luteis, punctis nigris instructis.

WSingarne unbertill antisı̧en affegriagtiga eller fötanbe $i$ mörf= gråt med ett fwartiunfterabt, init af farpt winflabe, fusarta linier

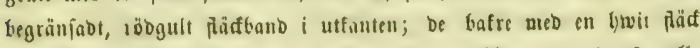
näta betta banb, gubilfen går intill en jammantyängande rab af ocell= 


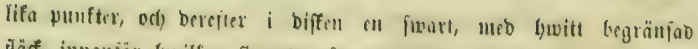

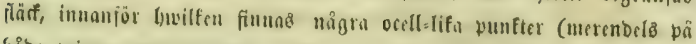

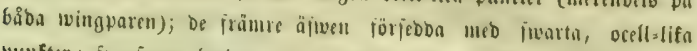
punfter; franjarue buvita. $\ell^{2}$ e. wingip. $1 \frac{1}{8}-1 \frac{5}{16}$ t.

Syanuc. BBingarne ofman $4 a$, fötanbe $i$ violett, med en ganffa fin, fwart linia uti utfanterne.

Soun. Wbingarme ofwan bruma, i biffen mer eller minbre blå, ofta förjebde meb gula, fwaripunfteribe vieller i utfanter.

Syn. Pap. alexis W. V. 184. Näbn. Pap. t. 60. f. 292-294. Trixt. 48. 19. Iyeana Ochs. Scim, I. 2. 38. IV. 25. « 18\%. Treitsch. 1. 1. 69, II-S. 1. 52. 1. 246.1 .74 .1 . 362 . Pierh. p). 27. I. 4. Zephiyrus Dalui. P. Ss. 98. 21. Argus Lett. Ins. Lapp. 913. 6. Polyommatus fiod. nt ilup. Lepid. I. 212. pl. 11 secund. f. 3. Pap. adonis Thby. Diss. II. 39. Ilesperia Palsr. E. S. III. 1. 299 . 134. (Var.) Pap. Iler/is Lisp. Schum. I. 1. 32. Suppl. 8. I. 2. Pap. icarius Esp. Schur. I. I. 3̈0. Sирpł. 26. f. 2. 3. p. 387. Pap. polyphe'mus Esp. I. c. 1. 55. c. 5. 1. 5. p. 31 .

Donna Bhlâninge är allmän i Gyla Gfanbinavien på alla bögg länbta ängar ody betesmarfer, fant på åfrar. Whot norben aftayjer ben

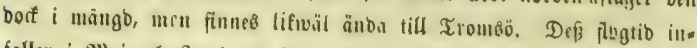

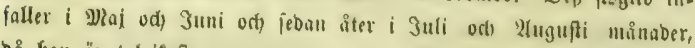
bå yall är talrifaft.

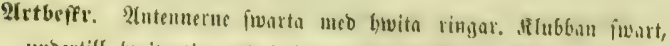

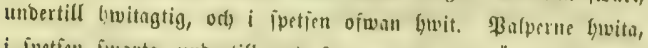
i fpetfen fwarta, unbertill meb fiwarta fticfelbår. D̈gonen furarta,

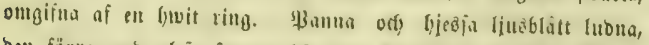
ben förra med glefa fwarta bår wib fiborme af ägonem. Nugg: ffölden ods afioomen ofuran blilubone. Den febrate unbertill

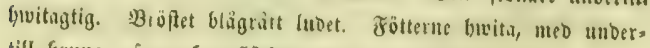
till bruna, ofwan brumpläfiga tarjer.

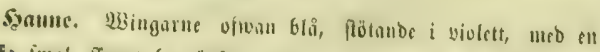
ganfa imsl, fearpt begränjab linia $i$ utfunterne. Framusingare nce fräure fant fhvit. Wiltgarne unbertill affegriagtigu, be 
bafre wio la a gen grönagtiga. De främre hajwa i utfanten twenne raber, med ot)bligt butitt omgijna, fivarta pärfar, lıwilfa lifwäl äro matta od lyajwa en ämmu mattare od) ofybligare antyoning

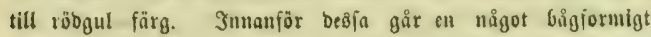
böto rad fwarta, meb bivitt ougifna punfter, of lywilfa be tเvenue närmaft infanten, huvilfa flunbom jafnas, iffe fth $i$ famma böining, fou be öfriga. WBio biffiältets flut finues en fwart, fort frrerf, jom lifalebes ăr omgifwen meb hroitt. Jumans för Deทกเ finnå̊ merenbels en efler twå, fwarta, meb lywitt omgifna puntter. Bafwingarue lyafn'a länge utfanten en rab röbgula fläcfar, fom utât äro förferbe meb fwarta punfter od, inåt begränjas of fearpt winflabe, fwarta frref. Frain mib=

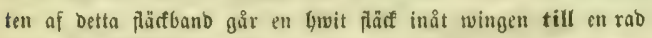
firarta, med buitt omginna punfter, of Grilfa de treme förfta

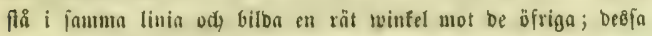
åter bafma närmare analğörnet en fröfning inåt od feban utåt.

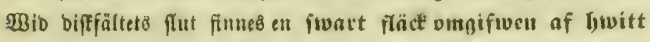
od) innanfor benna, wid wingbajeı $3-4$ jmarta, lifajtora punfter, fom filba en rät linia med lywaraubra. Särmaft franjarne loper en fmal, brun linis. Franfarne äro ofwan till inbre bălften bruna odj till ben sttre lyvita; men unbertill nă= fan enfärgabt brvita.

Syouau Vifnar i tefuningarne bannen; men är unbertill

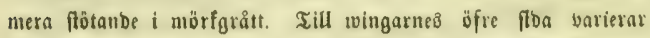
Lyon juart fagt $\mathrm{i}$ oänbliglyet. 3 allmänhet år bet röbgula fläct= banbet $i$ utfanten of twingarnes unbre flon lifa farft uttrhat på be främre, jom tå be luafre. Föllanbe äro be wigtigafte fårgfื̈rånbringarne :

1. Wingarne oiman mörft violetteli meb ett fwart twer=

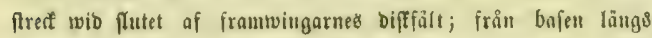

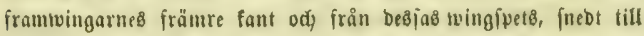

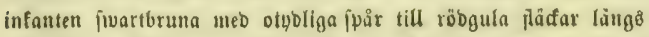
utfanten. Bafmingarne från bajen längอ framfanten füga brebt

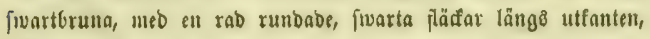


b)wilfa bwarbera Gafwa imnanför flg en trefantig, otsblig, mörf ftugguing.

2. W3ingarne oftwan jwartbruna, Glott $i$ biffen mörft violet. blå, me’ röbgula päcfar länge utfanterne, belo blott på bat= wingarme, bels äfwen på frammingarme. Rängs utfanten af be förra går, utanför be röbguła läđarne, en rab fmarta, runbabe

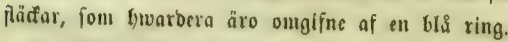

3. W3ingarme ofwan juart6ruma meb ent violettblå fläa i biffen, antingen blott bå framtvingarne eller of beriemte på bafwingarne, ody med en rab meb blätt omgifne, fwarta funfter längo utfanten af be febnare.

4. Wingarne oftwan mörffruna med bla anftręfning twio Gafen. För öfrigt Jif var. 2.

5. Wingarme ofwan mỏrtoruna utan fiăđår.

garveu, fom lepiwer på Ononis, Astragalus od̆ liragaria, ăr fint bårig, grön meb Juvita, wågformigt ajorutua, otpbliga

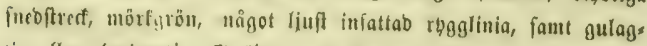

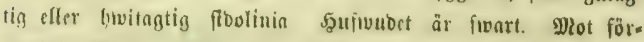
Ivandingsftuibent fötloras be wägformiga linicrne; larven fpin. nex of in fig. I nöbfall iörtär oen fona lifar. Dian fine net ben äfnet på Mlolilotus odh på Genista. \$hupan är glän. fanbe brumgrön med mörfare tagglinia od) gula luftgåt.

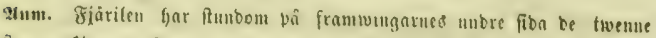
imerita mufterna af ben dielformiga twerraben fammanfutua meb sumf: terne wib bafen, fä ntt berigenom bilbas elt Gnlfcirfelformigt fluart, meb lglvit? begränfabt ftreff. Jjos fíbana exemplax äro berjemte teffuingarne af wingurmes unbe fiba grofre, an goe ben twantiga formen, meb grvilfen man tråffar bem tilliamman.

\section{Gingt-gBhivingen (Lycana adonis W. V.)}

Alis infra cincres fascia marginali, maculari, nigro-punchata ot lineis nigris acute anqulatis intus torminata, fulva, macula posticarum ante huju, modium alba, ad sorism contimuan punctorun ocellatorum adscomlento, maculaquo discoidali nigra, 
albo-martinala of puncles hasalibus ocellatis; anticis proterea

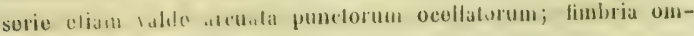
nium albá, nigro-maculati. L. al. oxp. $30-35$ millim.

Mas, Alis supra lælo courulois linea tenuissima marginali nigra

Femina. Alis supra fuscis, disco plus minus cooruleo, sape veellis intramaryinalibus, punctis nigr is instructis.

Wingarne unbertill affegrả neb ett fuartpunfterabt, inăt aj

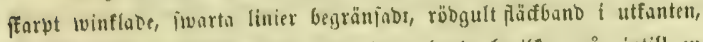
en buvit päsf på Gnfuimgarme nära betta bonb, Guilfen går intill en fammanbängande tab af ocell=lifa vunfter, ods berefter i biffent en frrart, med bivitt frigrinjad färe, innaniör fovilfen finnas några ocell-lifa punfter, (merenbelo pai bảba wimpluaren); de främre bef:

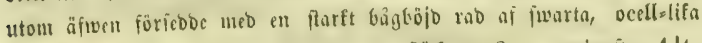

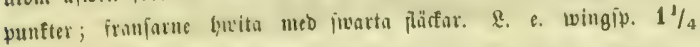
$-1^{7} / 16$ t.

sanunc. Rzingarne ofwant liubbla med en ganfa fin, fuart linia uti utfanterne.

Svona. WBingurne ofwan bruma, $\mathrm{i}$ biffen mer eller min= Dre blii, ofta föricoona med guln, fwartwunfterabe oceller $i$ itfonten.

Syn. Pap. adonis W. Y. p. 184. IÏ̈bn. 1. 61. 1. 298 -300. 1. 138. 1. 698. 699. of 1. 127. 1. 645. 646. Hesperia Vabr. l. S. III. 1. 299. 134. Tyerena Ochs. Schin. 1. 2. 33. ol 239. IV. 2\%. of 14i, Treitsch. X. 1. $6 \%$ II-S. 1. 52 1. 248. Gerlated jh. 30. I. 1. Zephyrus

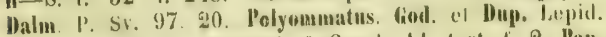
I. p. 210. pl. H. secuid. I. 2 pl. 11. telt. I. 2. Pap. ceronus Esp. Schm. I. t. 90, c, 40, f. 2. p. 178, 1. 102. с. 57. 1. 1. p. 50. Ilübn. เab, 6. I. $295-297$. Pap. Gellaryus Esp. І. c. เ. 32 suppl. 8. f. 3. p. 333. i. 55. c. ร. f. 2. 3. 4. 6; p. 30 .

Denn art ät bögกt fälignt inom Sfanbinatien. Rifwäl ä

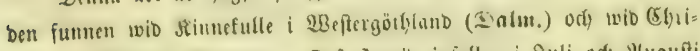
ftimia $i$ Jorige (E⿱宀⿻三丨口 månaber. 


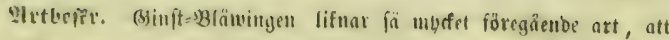
bär endaft ât nöbigt uppröra ffilfagtiglestexnc. 2futennflu6ban är unbertill od) $i$ ipetien röbgulagtig. Det röbgula fäađbarbet på bufwinjarnes unbre floa är minbre än bos föregåente ods läafarne mera tillipetfabe; ben bervåfülianbe fäăraben är längre aflägẹn från banbet, än 1908 meranãmnde art ợ på frameingnrue ftarfare bâgbölo. W3io bafen af irambingarne

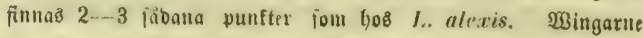
äro ofwan bos banuen lfuft od) mafert blá, jamt genomiópa

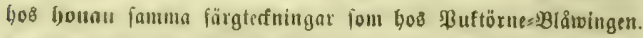
Franjarne äro hos båba föntu rent bwitu meo fwarta fläăar, Der nerverne hafwa fitt utlopp.

Saryen lefwer pâ blomptren af Trilolium od Genisla.

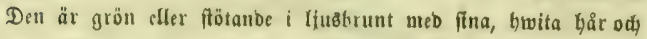
en mörf rugglinia, loid bwarß båba fibor går en rab, trefans

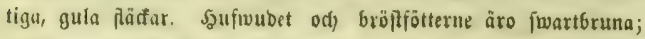
bufötterne grönagtiga. \$\$npan är trubbig, grön eller mỏrf. 6run. (Dd)i.).

\section{Honingsklifiver-b3åvingen}

(Lycæena dorylas $\mathbf{W}$. V.).

Alis infra cinereo-fuscescentibus marginibus late albis, fascia maculari intramarginali sub-nigro-punctata et lineis arcuatis nigris intus intordum terminata, minuta, fulva, macula posticarum ante bujus modiun alba, seriem continuam punctorum ocellatorum transcendente, maculaque discoidali anticarum nigra, albo marginata, posticarum alba, ot punctis nigris basalibus in anticis nullis, in posticis minutis, ocellatis; anticis proeterea serie otiam sat arcuat: punctorum ocollatorum; fimbria alba. L. al. exp. $32-40$ millim.

Mlas. Alis supra coorulois margino tonui, haud doterminato, ot extromitatibus nervorum nigris.

Femina. Nis supra fuscis maculis marginalibus fulvis. 
DSingutnc unbertif affegråantigt bruma, i utfanterne brebt Gitoita meb ett imartpunfterabt, inăt af Gågföiba linier unberfunoom

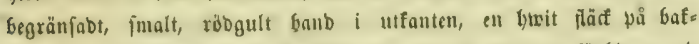
twingarne uära betta banb, gurilfen går in ät wingen förbi en rab af ocell=lifa punfter, ody berfter i biffes on på frambingarme fwart

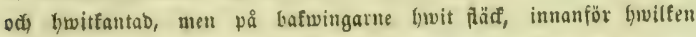

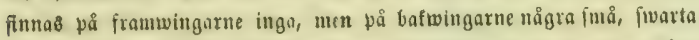
ocellelifa punfter; De fräure befutom förfedba meb en temligen Gåge gadto rab af fwarta ocellslifa fläđar; franfarne lywita. \&. c. twingfip. $15 / 16-1 \%$ t.

Scaunc. Wingarne vfwant ljuşlå meb junal, fügn feegrän= fab, fwart utfant od $i$ bittre älbarne froarta nerver.

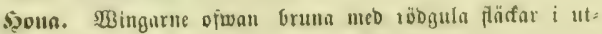

fanterne.

Syn. Pap. dorylas W. V. p. 322. Nlibn P'ap, 1. 60. f. 289-294. Ilesperia Vabr. Li. s. III. I. 299. I3.). Irycæna Ochs. Schm, I. 2, 31. IV. 25. ol 145. Treitsch. X. 1. 67. ॥-S. t. 74. I. 363. Gerh. pl. 30, f. 3. Polyommatus God. ot llup. Lepid. Suppl. I. pl. 12.1.1-4. Árgus Boist. Icon. p. 54. pl. 14. I. 1-3. Pap. hylas Esp. Schm. 1. 1. 45. Suppl. 21. f. 3. p. 375. Pap. thetis Esp. I. c. t. 33. Suppl. 9. 1. 3. p. 335.

Üran at Lafwa upptäft inom Gfanbinavien benna ast tilltör

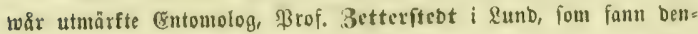

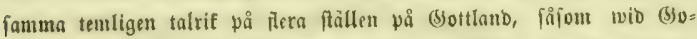
them, på ₹årön, Thorsforg odh Furillen. Gedun har ben blifwit

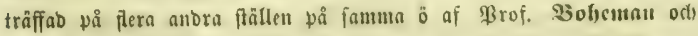

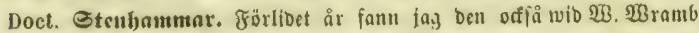
od) 2̊rup $i$ Gfåne. Deşa äro be enba lofaler iör artens förcfomft

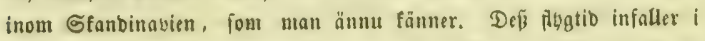

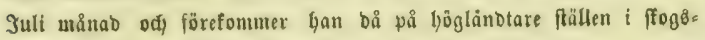
trafter.

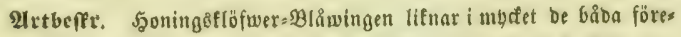
gåenbe arterne, få att bet är tillräafligt uppföra beß ffillagtigs beter. 2(ntenner, palper od ojriga froppsobelur jåjom bos ßufo to๊rnes 8 ในำingen. 
Sanme. BBingurne vivan liusblif meb imal fwart utfant, font inht of ä ffarpt aiffilo fran grumbiagen. Winguerverne, ifthmerbet poi frambingarne, nânmaft utfonten iwarto. WBingarne unbertill affegringtigt=bruma uted inmma fwarts tefning fom hoz Buftörne-Blåmingen; men utfunterme äro fred brvita ody ber Deına Gwita färg flutar finnas iun trefantiga, från brvar= anora temligen wiot ffilba, röbgula fläcfar, fou ftumbon bafusa utunför fig en a ad tybliga fivarti punfter, men vitnft ganffa fmå od) otmbliga libana, faut inåt meta fällan begränjas af pwarta

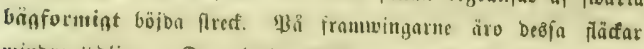

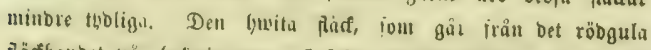

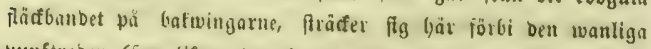
Junftruben (fom lifnur ben bos L. aleris) iram mot biffläcen, buvilen ben lifwäl ef bimner. Denna är rout buvit, lifertfor= mig, od) jänber en liten frimma lnfảt. \$Bi frummingarme år

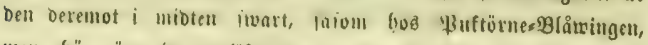
men l)är äto be ocellformiga punfterne fòrre än på bafmin. garne. Wio bafa af iramuingarne fimnas imga oceflslifa punfter; tå Gafwingarne bereutot $2-3$ imärre. Franjarme äro rent bnita, merenbela ofman i inore bäliten cubaft på fram= wingarne bruna.

Soman lifnat linnen till wingatues unbre fton. Rifwal är bet töogula fläfbanbet metcubeles ftörve od äfwen tybligt på jrantwingatus. Diwan äro tvimgarne fruma, något glänfanbe,

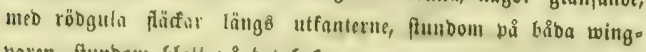
paren, ftunbom blott på bet bafre.

Sarven, fom Icfiver bå blomftren of Molilotus, är fint lu= Den, mörfgrön, något fitutanbe i brunt, med en wacfert gul fioo. linia od) mörfgrön, fin rbgglinia, pa btwats båba ftoor går en rao gula flärfar. ફ̧ufwubet tentigen fơrt, fwart, lifufå lujis båter.

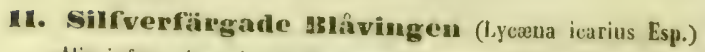
Alis infra cinerois basi coorulosconto, fascia posticarum 
marginali maculari minuta nigro-punctata of linois nigrris acute angulatis infus terminata, fulva, sorio communi continua punctorum ocellatorum, ot ante illam macula discoidali nigra albomarginala, punctiaque sparsis ocullatis ad basin posticarum; fimbria alba. L. al. exp. $30-35$ millim.

Mas. Alis supra argenteo-couruleis margino lato, indeterminato of extromitatibus norvorum nigris.

Femina. Alis supra nigro-fuscis disen plus minus cooruleo, posticis punctis marginalibus nigris, intordum fulvoocellatis.

2Bingarne unbertill affegrå, wib bafen Glångtiga, meb ett fumalt, fwart punfterabt, ina of fforpt winflnbe, fwarta linter begrảnfabt, röbgult fâđđGand $t$ utfanten, intill Gwilfet gi̊ en gemenjam, fams manל̧añnanioe rab jwarta, ocellslifa punfter, orly inom besma en fwart,

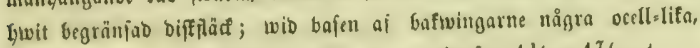
furibba puntter; franfarne byita. \&. e. twingip. $1 \frac{1}{4}-1 \frac{7}{16}$ t.

Same. WBingarme ofwan fllfwerfärgabt blå meb breb fmart, inåt obeftämb utfant ợ $i$ pttre änbarme fwarta nerver.

5oun. Mingarme ofwan fwartoruna, i biffen mer ellet minore blå; be bafte neé frrarta puntter $i$ utfanten, och fluns bout robgula oceller.

Syn. Pap. icarius Esp. Schu, 1. t. 49. c. 54. f. 4. p. 35. Jyexna Ochs. Sclım. 1. 2. 37. ot 239. IV. 25. Treitsch. X. 1. 68. Zephyrus Dalm. Pap. Sv. 97. 19. Polyonmatus Goul. ot Dup. Lopid. Suppl. I. 69. pl. 11. f. 1. 2. Argus Bolsil. Icon. p. 50. pl. 12. f. 1-3. Zett. Ins. Lapp. 912. 5. Pap. amandus Illälon. Pap. 1. 59. \{. 283-285. Pap. corydon Thbg. Diss. II. 39. Lyycrena Gerh. pl. 29. r. 4.

Dentก $\mathfrak{B l a ̊ m i n g e ~ f o ̈ r e f o m m e r ~ m i n o r e ~ a l l m a ̈ n ~} i$ fäbra od utebs lerfta belarne af wår halfะỏ. Delt är aumărft inom Sfåne, wib

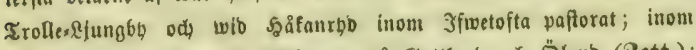

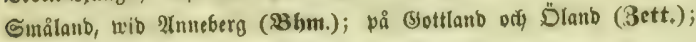




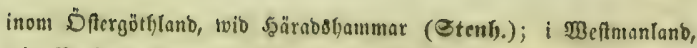
wio Norberg (Frrebrichf.). Эnom fabra Norige funes ben beremot

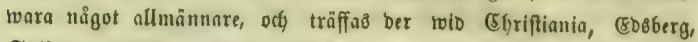

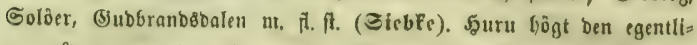
gen går mot norben fänner man ef med wişyet. Dod är bet troo Itgt, att ben ef firnnes inom \&appnarferne. Dé figgtib infaller i

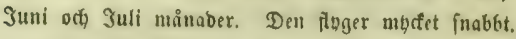

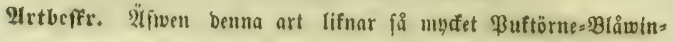
gen, att wi bär enbait anföra ftillagtigheterne.

Sanuc. Wingarne äro ofman flfinerfärgabt blå me tem. ligen brob, fwart utfant, bwiffen inåt uta॥ beftämı färggräns อ̈ศพergåx i grunbfärgen, fom närmaft utfanten genonifãres af De berfäbes fwarta nerberne. Unbertill äro wingarne ạfegra?, till betoblig brebo wib bajen, ifpnnerflet på baftwingarne, Glå. agtiga. De främre fafua i uffanten röbgula läafar od bुafma blott några mörfa ffuggningar; bernåft fölter en ftarft fölo rab af be wanliga ocellslifa punfterne, Guvitfa Gär ofta äro tem. ligen fuเå, ợ flutligen ben wanliga biffiäađen, innanför bwil.

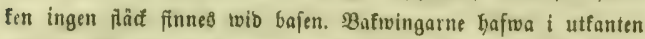

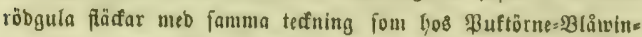
gen, men be âro här mbcfet imärre ody till en bel otgoliga;

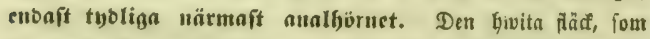
föregåenbe 3:ne arter ega wib betta banb fafuns fyär allbeles, och finnes idfe ens antbob. Den ranliga raben of ocells lifa punfter lifuar ben hoo Puftôrne: Blůıingen, uten gör enbaft en föga märf6ar frỏfning närmare analbòrnet, fâ att ben här är rätare, än Łos någon of be 3:ne föregåenbe arterne. Diffes flärten lifnar ben yos $L$. alexis, äfweniom be wanliga punt: terna wib bajen.

Souan år ofwan fwartorun meb mer eller minbre blatt $i$ Difen. Bafningarne bafwa uterenbels en rab fwarta punfter längs utfanten od) ftunbon äro beకీ |a omgifne af röbgula rin. gar, få att be lifna oceller. Undertill lifnar bonan allbeles byannen, endaft meb den flilnab, att be röbgula färfarne äro på 


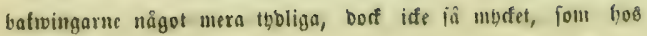

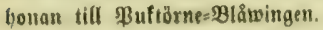

Qarveu fall leftua på Fragaria (Serolo.)

\section{Bakvingarue undertill med svarta punkter och utan röllgula flickar i utkanten.}

A. Ögonen nakna.

12. Srarthäckign Mlâvingen (Lycæna arion lin.)

Alis infra canis, ad basin rirescenlibus, punctis nigris marginalibus hi-eriatis disfinctis. sure valde arevafa punctorum ocellatorum, macula nigra discoirali albo-marginata punctisques ocelfaribels spar: is ad bavin; fimbriis albis, infra nigro maculatis, I, al. exp. $40-45$ millim.

Mas. Alis supra coeruleis margino lato punctisque anlicarum discoidalibus nigris.

Femina. Alis supra corruleis margino latissimo punctiscuo anticarum discoidalilus majoribus nigris.

2Bingarne unbertill is̊grå, rib bafen grönagtiga, meb twenne raber fwarta, tybliga punfter i utfanterme, en ftartt Gågböjo rab

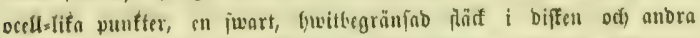
furibba ocellslifa punfter wid Gafen; iranfarme buvita, anbertill fiearte

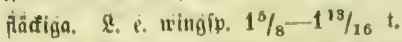

saame. WBingarme ofwan bögbli med breb, fwart ufant od friarta puntter $i$ biffen of framubingarme.

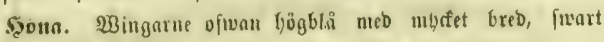
utfant o(u) förre fixarto punfter $i$ biffen af fratumingarme.

Syn. Pap. arion Lin. F. S. 283. 1073. Ilesperia fabr, E. S. III. 1. 293. 118. Papilio llïbn. Pap. t. 54. 1. $254-$ 256. Texi. 44. 2. Jyerena 0chs. Sclin. 1. 2. 4. IV. 24. Treitsch. 1. 1. 58, Wephyrus Dalm. P'up. Sv. 94. 13. Polyonmates, God. of Bup. T.opid. I. 219. pl. 11. f. 2. pl. 11. quart, 1. I. Lycaena Eerh. pl. 33. I. 3.

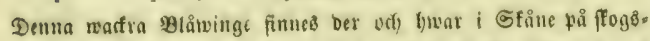

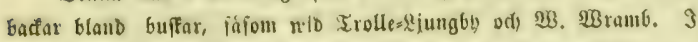
slefinge bar lag funnit Gonom temligen almån wib Gỉlvegtorg. 
Bå Bottlanb finnes ben på fleri ftällen, fäfous trib Wamblingbo od

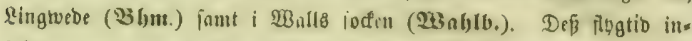
faller i Suli ody bejöfer fiärilen ifjunerlyet bi blommande arter af

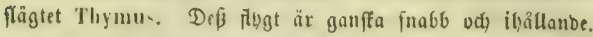

Alrtbeffr. 2Intennerne inarta med fwita ringar. Sllubban fwart, $i$ ipetfen lusitagtig. Falperme Gusita, $i$ ipetfon frorta, unbertifl med fitarta ftiffelthir. D̈gonen bruna, gulbglänfanbe, omgijne af en lyvit ring. 5ूufnubet mellan ögonen fwartlubet meb en

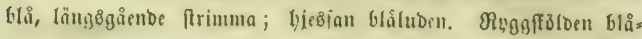
luben. 2lboumen viman fwart med glefa, blå bår, undertill o(d) på fiborne flipwergrå. Fötterne hwita med brunringlabe tarjer.

Sanume. WBingarne ofwan l;ögblå méb breba, fwarta uts fanter. De främre hajwa mid biffältets flut ett fwart ftrect odi) mellan betra famt utfanten flera efler färre, parallelt med wingnerverne gåenbe flörre elles minbre fwarto fläfar, ywilfa tillfamman bilba en båge. Stunbom, cluru mera fältan, finnes

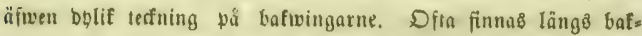
wingarnes utfunt jwarta, med bliitt umgíma punfter. Hnoers till äro wingarne ięgrå, wib bafen, ifpunerbat på be fafre,

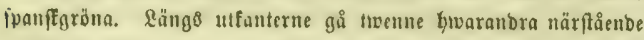
raber af tybliga, juarta pärfar, obetubligt fantabe med lywitt. Difwer bifen, af blvarbera twingen gair en rab jwarta, meb h)vitt omgina bunfter, lywilfa äro förft pi̊ framuringarme, ber de bilda en farft böld bigge (nâftan en Galfcirfel); $1-2$ af be inbse funfterne fta ef $i$ famma rab fout be öfriga, utan utan=

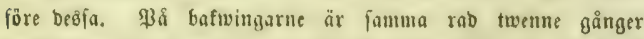
flarft bülo, få att ben, börianbe wid framfanten gör ett farft fprång utåt, berefter stt minbre inåt od) feban åņ⿻ utåt, fört

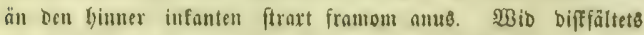
flut funnç pa buarbera wingen ett fwart, Lywitbegränfabt fored od) innanför betta 1 -..3 jwarta, af bwitt omgifna punfter wib winglajen. Sälln fafuns besía belt od bållet po̊ frammin=

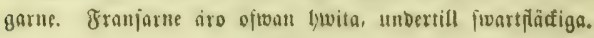


Syonan tifnar $i$ bet närmafte banuen, men f)ar på wingar:

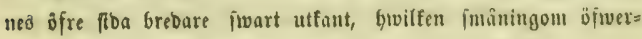
g̊̊ $i$ grunbfärgen, inmt merenbel ftôrre fwarta plädar.

\section{Punlsterade IBlívingen (Lycantit alcon W. V.)}

Alis infra fucco-cinereis punctis nigris marginalibus hiseriatis obsoletis, serie arcuata punctorum ocellatorum, maculaquo nigra discoidali albo-marginata; posticis ad basin aut impunctatis aut punctis ocellatis sparsis; limbriis albidis, infra uscoscentibus, L。 al. exp, 95-40 millim.

Mas. Alis supra coerulois margino tenui nigro.

Femino, Alis supril nigro-fu-cis disco subcoeruleo punctis obsolelis nigris.

Wingarme unberlill brunagtigt affegrå meb twemme raber fiwarta, otybliga punfter $i$ utfunten, en bigböid rab ocellelifa punfter orle en fwars, bwitbegränjabo flärf $i$ bifen; be bafre wib bajen antingen vpäfabe eller of meo ucellifa, fpribba punfter; frallfarne busita, un= bertia brunagtiga, \&. c. wingip. 17/16-1\%/8 t.

şaute. Wingarne ofwan Glå med faral, fwart utfant.

Sona. Wingarne ofwnn fwartfruna med ï̈ga blå bift od otboliga, froarta punfter.

Sjn. Pap. alcon W. V. 132. Uübn. Pap. t. 55. C. 263-265. Text. 44. 4. Hesperia Fabr. E. S. III. 1. 293. 120. Lycana Ochs. Schu. I. 2. 7. IV. 25. Treitseh. $\lambda$. 1. 58. Zephyrus Dalm. Pap. Sv. 94. 14. Polyommatus God. ol Bup. Leprid. Suppl. I. pl. 50. f. 1. (liu. incorr.) Argus Boisd. Icon. p. 81, pl, 13. f. 1- - Ilesp. argindes Palor. 1. c. 300 . 138. Pap. arcas Esp. Sclm. I. t. 34. suppl. 10. f. 4. 5. 1. 338. Lyeana Gerh. J1. 32. f. 5.

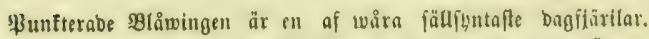
Den förefonumer på fuftiga ängar ber oḑ bmar $i$ Wgefter= od) D̈fter. götblanb. Snom förra provinfen är den trăffad wio Simufulle

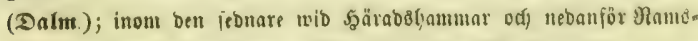
bällsberget wib Söbertöping (Etent).). Deß̧ Fingtio infaller $i$ feb

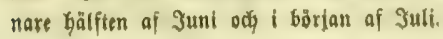




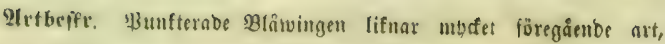
men abbomen är unbertill brunagtigt grå.

Sonue. QSingarme ofwan föggla, utan imarta punfter od fiärfar, men med fual, fuorf utfant. De fräure från bafen,

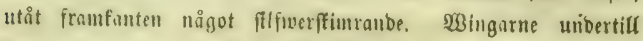
brunngtigt affegrio mcb ett liswitngtigt ftred ocy trwenne, bwars anbra närftienbe raber of mattn, otybliga, fwartagtiga flädar

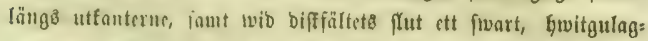
tigt omgifwet fitert. (Ëmellan belta od) utfanten går en rab af jurtar, byvitgulngtigt omgī̄na puntter, Gwilfa på Gafningarne löpa till infunten $i$ en mora rät linia, än fos föregående art, feDan be lifüil trid framtanten biloat en båge. ßå framwin.

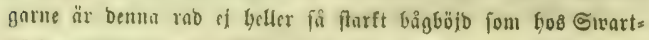

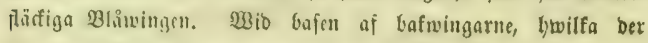
ï) obetudigt anlukns med ipanffgrönt, finuas $1-3$ fwarta,

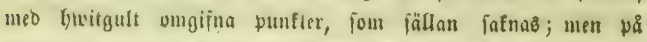

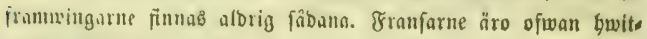
agtiga, undertill biunagtiga.

Sovinu, fou till wingarnes unbre fiba lifnar hannen, yar

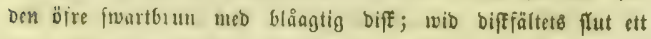
(iwart ftref och) cmellan betla famt utfanten en böld rab af vtybliga, fwarta punfter.

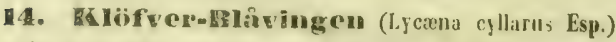

his inlra lectu cinereis, ad basin late viruscentibus, macula discodilli nigra albu-marginati, sericquo punetorum ocollatorum, in posticis minorum. L. al, exp. 30--35 millim.

Mas. Alis coeruleis, micantibus, margine tenui, ad apicom latiori, nigro.

Fimina. Alis nigro-fuscis disco coeruleis, macula discoidali nigra.

WBingarne unbertill lluft affegri, wid bafen brebt grönagtiga,

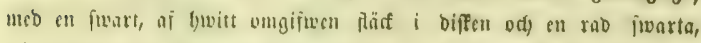
på luftwingarme funa, ocell=lifn puntter. \&. e. mingip. $1 \frac{1}{4}-\tau / 20$ to 
Syaute. Wingarne glänjanbe blå meb imal, ntot uxing. fpet [en brebare, fwart fant.

Sgoun. WBingatme iwartfruna meb blå biff ody fwart flät 1 Differt.

Syn. Pap. cyllarus Esp. Schur. I. T. 33. Suppl. 9. f. 1. 2. p. 334. Hesperia Mabr. E. S. Ill. 1. 294. 122. Iycana ochs. schm. 1. 2. 12. IV. 25. Gerh. t. 15. I. 3. Zephyrus Dalm. Pap. Sv. 95. 15. Polyommatus. Fiod. ot Dup. Lepid. 1. 222. pl. 11. f. 3, pl. 11. quart. f. 3. Pap, damoetas llübn. Pap. 1. 56. ケ. 266-268.

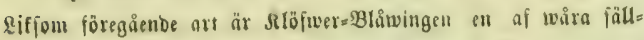

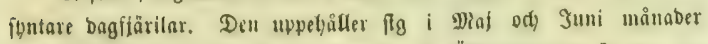
belft på ängsbađar, ber efeftog finues. 3 D̈ftergütlland är ben tas

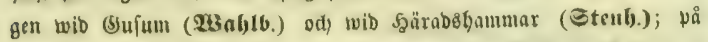

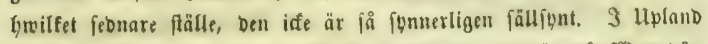

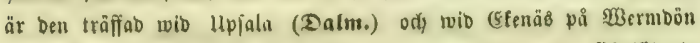
(IBablb.). Snom গorige är bell anmärft wid Ebriftiania (Siebte).

Artbeffr. 2(ntennerne fwarta med fywita ringar. Slubban mtöt: brun, ofwan twib fpetien hwit. \$alperne blågtigt bwita meb iwart ipets, unbertill med fwarta ftifellyår. Dgonen ods hufs

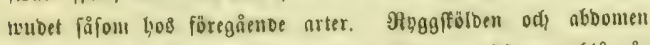
Diwan blålubne. Den febnare unbertill bnit. Fötterme blågrå; tarferne meb fwartagtiga ringar.

Sonuse. Bingarne ofwan glänfande blå meb intal, mot ipetfen brebare, fwart borba $i$ utfonten. WWingarne unbertill lijuf aftegrå; be främre i biffen meb ett fwart, af hivits onlgifwet fired od utanför betta 5-6 fwarta, meo hrwitt omgifna punfter, fom biloa en bojib twerrab; ifmnertyet 2:ne af beชิ fa

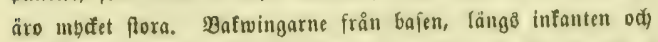
utofwer uribten fpanffgröna, med ett otybligt, fwart twerftred wio biffältets faut ody en twerrab af imai, fwarta, af fwoitt ougifna punfter, af bivilfa $1-2$ ftr trio framtanten, widt ftilba från be äriga; beşia fanbemeilan olifftorn, jå att några af bem fnarare lifna atomer än punfter. \&ängs utfanterne af 
wingarne löwer en fmal mörfbrun linis intuib be lysita franfarne.

Syouas lifnar fyannen till twingarnes unbre fiba meb un= bantag beraf, att ben ẩr mera ftötanbe $i$ brunt. Mingarne äro ofwan fwartbruma, från bafen utofwer biffen blå, meb et fiwart

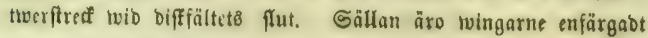
Eruna på offre fiban.

Snrucu, fom lefwer på Trifolium, Astragalus, Gonista

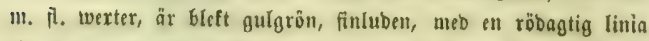

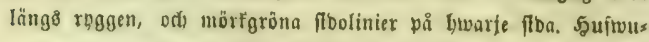
bet o(t) bröffïtterne äro frarta; abbominalfötterne grönagtigt bruna, æupau är Erunagtig.

2/nm. Fjảrilen ljar ftunbou tri bafrvingarnes unbre fiba blott on veellsfif (fwart, meb fowitt omgifween) puntt.

\section{Nyelvicke-HBlivingen (Lycena pherotes llubu.)}

Alis infra cinereis, anticis macula discoidali nigra, albo marginatu, seriequesubangulata punctorum ocellatorum; posticis serio duplici macularum albarum. L. al. exp. 25-30 millim.

Mas. Alis supra cyanei margine tenuissimo, indeterninato, nigro.

Femina. Alis supra luseis, lineola discoidali obscuriori. W3ingarme unbertill affegri, be främre met enl fwart, buttbes

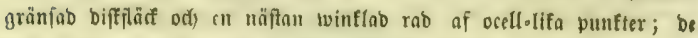
Frafre meb en bubbel rab of brvits fläfor. \&. e. wingip. $1-1 \frac{1}{2}$ t.

Sautu. WBingarne ofwan bla mé gauffa fmal, inåt obes gränf̧ab, fruart utfant.

5oun. Wingarme ofnan mörfbruma med elt litet, mörfare firent i biffent.

Syn. Pap. pherefess Hïlon. Pap. Trexe. 15. 8. Lycaena Ochs. Schm. I. 2. 25. IV. 25. Mierh. pl. 22. 1. 1. Polyommatus. God. of Bup. Lopid, Il. 202, pl. 25. Г. 5. 6. Aryus Zett. Ins. I.app. 912. 3. Pap. alys Esp. Schm. I. t. 118. c. 73. f. 4. 5. p. 10. llihbu. Рap. t, 97. i. 495. 496.

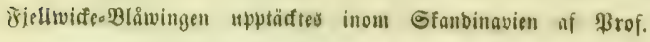


Soheman 1832, unber hans Morfa refa. Dell fanns of fyonon ifุ̧nnerbet allmän på norra flutmingen af Dowre fiell wib (5äftgifs waregårben Bhilfe, od feban wib Drioftuen unber iebnare bäliten of Sunt. Sebermera bar ben ăfwen blifwet funnen i (Subbrandébalen

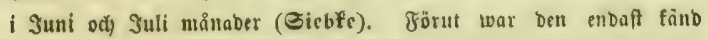

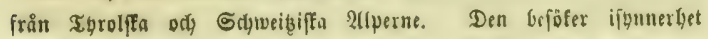
Blomftren pน̊ Astragalus alpinus.

Artbefer. Antennerne ofwan jwarta meb frwita ringar; unoertill beritagtiga med bruma ringar. Rlubtran jwatt, ofwan med en broit twerfrimma nära fpetjen. Balperne gråatiga, mot fpets

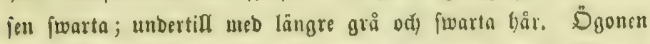
fwarta, ougifna af en frwit ring. Şufwubet mellsu ögonen

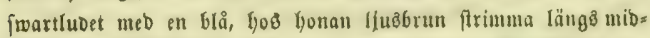

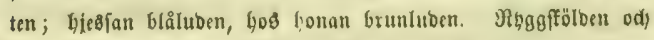
abbomen Gos lyannen blålubua, yos fyoman brunlubsa. 2660= men unbertill, lemte Gröftet, gråluben. Fötterne Glwitgrå; tar jerne med bruna ringar.

Saaumc. Wingarne ofwan ljuøblå meb fmola, jwarta uts fanter, h)tilfa inåt ef äro fearpt afftilba frin grunbfärgen, fom nårmaft ufanten genomftäres af be bär jwarta tringuerberne. Frantwingarnes främre fant fmalt, men be Gafres brebare fwart. Frammingarne unbertill affegro meb ett fwart, af hwitt omgif= wet ftreá wib bifffältets flut o(s) utanför betta en rab fwarta, hwarbera meb htwit ring omgifna, fmi punfter, af Gwilfa ben fỏrfta får innanför be öfriga, fom tillamman bilba en rät linia, Gwarföre bela raben funes winflad nårn framfanten. Baf= wingarne något nı̈rfare, wib bafen grönagtiga, meb twenne ra=

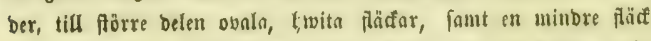
nära framfanten wib bafen. Den fåa, fou är Gelägen tvib biffälteţ flut, är något blertformig. Franfarme ofwan Guvita, unbertia gråagtiga.

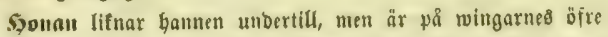
fiba mörfbrun med ett mörfare flterf wib bifffältets flut, faut wib wingbajen något blåpubrab. 
16. Mazurfïrgade mhinvingen (Jycena alsus W. V.)

Alis inlra cinerois macula discoidali nigra, albo-marginata; anticis serie punctormm ocellatorum subrecta; posticis serio punctorum ocellatorum alsrupta, sinuala, interdum deficiente punctisque ocollatis basalibus. L. al. exp. $22-28$ millim.

Mas. Alis supra fuscis, coeruleo inicantibus margine nigro-fusco, tenuissimo.

Femina, Alis supra nigro-fuscis.

Bingarne unbertil affegrå uted en froar, af bwitt omgifwen fäå $i$ biffen; be fränure beriemte me en näftan rẩ rab af fwarta ocell:Iifa punfter; be bafte med en bulif, men aftruten, Gugtig, flun.

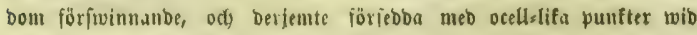
Eajer. \&. e. wingip. $16 / 16-1 \frac{1}{8}$ t.

Sanure. Wingarne ofwan bruna, bläfinuranbe, meb ganfta imal, fwart utfant.

50un. WBingarne ofwan fwartbruna.

Syn. Pap. alsus W. V. 184. Hesperia Nabr. E. S. III. 1. 295. 125. Papilio llïhn. Pap. 1. 58. 1. 278. 279. Lyeana 0chs. Schm. I. 2. 22. IV. 25. Treitsch X. 1. 65. Gierh. pl. 13. f. 2. Zephyrus Dalm. Pap. Sv. 96. 18. Argus Zeit. las Lapp. 912. 4. Polyommatus. fiod. et Dup. Lopid. II. 208. pl. 26. f. 5. 6. Pap. minimus Esp. Schm. 1. I. 34. Suppl. 10. f. 3. p. 338. Pap. alsoinles Gerh. 1. 13, 1. 3.

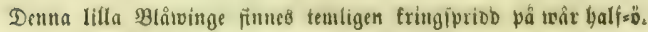

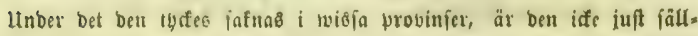
font $i$ anbra. 3 Sfine funtes bent der od) bwax, fäjom wid \&unb (3ett.)

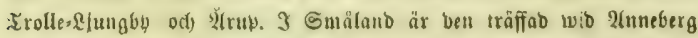

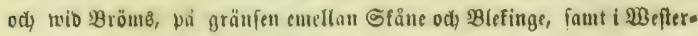

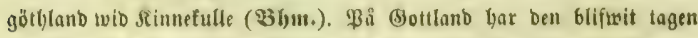

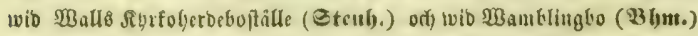
కnom Hpland fisneß ben temligen allmän tvid Upfala (Datm.) ođ̆ wib Stofflolm (23ablb.). I Norige är ben funnen uib (5briftiania (Sicbfe), wid Revanger ode 2llpaljang (3rtt.), î́ alt bin bär tb̆fes gå ganffa bögt mot norben. Snom Stoenffa Lappmarferne 


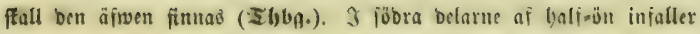

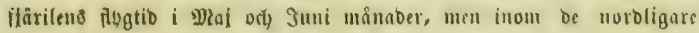

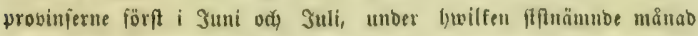
ben blifwit funten ivib 2uftabaug.

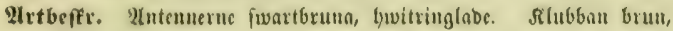
unbertill Gwitagtig. Balperne blångtigt bwita med fwartagtiga

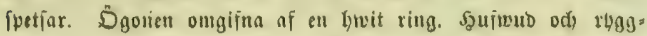

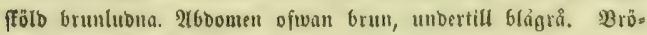
flet blågrått, lubet. F̧ötterne Gwitagtiga.

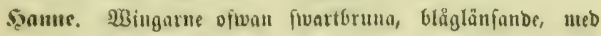
imal, jwart utfant. WBingarne unbertill affegrå med ett fiwart, af hyvith omgifnet ftrect wib biffältets flut, ody utanför benna ell rab, imå, fwark, of hyvist omgifma punfter. \$å framuvins garne åx benula rab näftan rät, enbaft förfta punften wio fraufanten får framom be öfriga. Đå faftuinģarne är ben beremot nårmate infonten bugtigy, iâ att ch punft flår långt inom be Dfriga, och wio framfonten är ben aforuten, fă att twenne

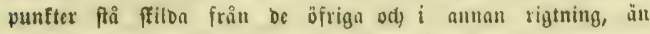
besia; De bilba nentigen en rät linia med biffiftedet, ftunbom jafnas tillodjumeb fiörfta belen af be putuftex, fom tillyöra benna rab. WBib bafen af bafivingarne finnas nigra punfter of famma färg, fout be förut beftrifna. Frtanianne ảro blwitgrå.

Sgouan lifnar bannen, men är pis wingarnes öfre fiba en, färgabt fwartbrutt.

Garven fall tefwa vå Trifolium (syecoto).

\section{B. Ögonen ludnu.}

17. Kugs-nhâingen (Lyexna acis Mchs.)

Alis infra einerois, ad basin haud virescontibus, macula discoidali nigra, albo-marginata, serinquo punctorum ocellatorum, anticarum arcuata, posticarum subabrupta, sinuata; hasi posticarum unipunctals. L. al. exp. 30-35 millim.

Mas. Alis supra cooruleis margine indeterminato of extremitatibus costarum nigris.

Femina. Alis supra fuscis. 
WBingarme unbertill affegrå, wib bafen obetobbligt grönagtiga, med en fwart, af hwitt ongiimen fläc $i$ biffen od, en rab ocellolifa

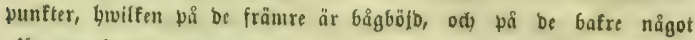
affruten, Gugtig; be fafres bas meb en liten punft. \&. e. ivingfp. $1 \frac{1}{4}-1 \frac{7}{10} t$.

Şauแe. WBingarne ofwan blå mcよ oliegränfab, fwart uts fant ody nerbernes yttre änbar fwarta.

5yoเa. BBingarme of wan frartbruna, enfärgabe.

Syn. lyesena acis 0chs. Schm. I. 2. 14. IV. 2\%. Gerh. pl. 13. f. 4. Polyommatus Gerl. at Dup. Lepid. I. 224. pl. 11. secund. f. 7. pl. 11. quarl. f. 4. Tephyrus aryianus Dalm. Pap. Sr. 95. 16. Irgus Zeti. Ins. Lapp. 912. 1. Pap. argiolus Hilion. Pap. I. 56. f. 269-271. Toxt. 45. 5. Hesperia Habr. E, S. III. 1. 295. 123. Lisp. Schm I. 1. 21. f. 1, a, b. p. 277. Pap. acis W. V.

Unm. Det ât blott for att gifwo wifa fôr bet alluảnt antagna brufet, fom wi här falla beuma art $L$. acis, ehuru gau meb rōtta borbe bän bet af Dalman sifnc namnet, emeban berigenom all collifitun meb fols janbe uubliáfoes.

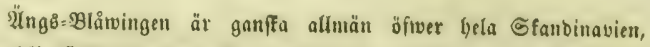
be norbligafte prosinjerme iffe unbantagna. Från Sunt till flutet af Ulugufti månaber fuger lyan i ffogar oḑ på ängar.

Irtbefex: 2lntennerne in'arta, lywitringlabe. Stlubtran imart, unbertill Gyvitagtig. Balperne bwitagtiga, i jpetfen fwarta, uns bertill med fwarta flicellfår. Ḋgonen guloruma, ourgifua af en

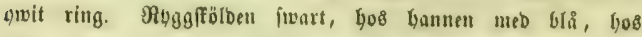
honan meb frum lubb. 2abomen ofwan livartagtig, unbertill G)witagtig. Brôftet Glågrått lubet. Fötterne brwita meb bruns. ringlabe tarfer.

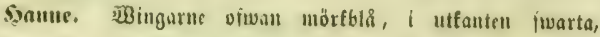

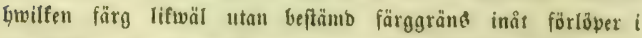

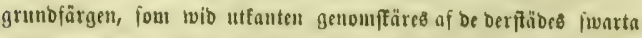
wingnerverne. Detterfta fromfanten af be främre lvingarne imalt bwit. Bingarme unbertill affegrå mé ett jwart, af Givitt omgifwet ftref $i$ biften odi utanför betta en rab 
inarta, lifalebes meb lywitt ungimu punfter. Denna lab är pä frammingarne temligen flarft bảgfüio, lifwäl fofnne ftunoom

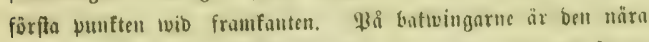

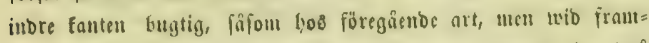

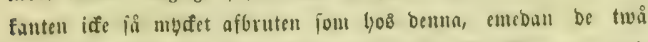
förfta punfterme ftâ bär ife i linia meb biffiftrectet, utant méb ben 4:be eller 5:te punften a famma rab. WBid bajen af fafs wingarne finnes nära framfanten $\mathrm{en}$, be ofrige lifnanbe punft,

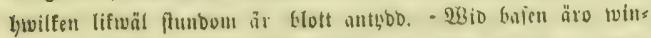
garue obetybligt grönagtigt anlupua, lifuril uкx pả oe lafse,

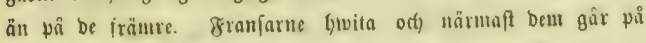
wingarnes unore flo en frual, frunagtig linia.

Syouna, fom till wingarnea unbre fidoa lifunt bannen, är till bell öfre fwartbrun, $i$ en wił̧ dager nâgot glầnfanbe, ợ flunbon fynea eft otyoligt, mörfare flerd wib flutet af biffältet. Franjarne åro brunagtign, i wingipeliarne hwita.

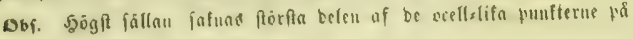
vingarnes unbre fiba.

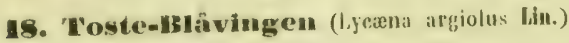

Alis info lactoo-canis macula discoidali nigra soriecyuo punctorum oblongorum nigrorum, anticarum subarcuata, posticarum valde abrupta, sinuata; basi posticarum tripunctala. L. al. exp. 25-35 millim.

Mas. Alis supra Into cooruleis apicibus anticarun tenuissime infuscatis.

Femina. Alis supra coerului, apicibus anticaum, margineque antico posticarum late nigro-luseis; posticis margine exteriori nigro-punctato.

Mingarne umbertill bläagtigt lowitgrî ueb en fwart flä $i$ bis fên och en rab af jwarta, aflånga punfter, Guvilfen pâ iramtuingarne

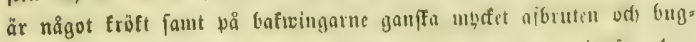
tig; baftwingarue med $3: n e$ punfter wib bafen. \&. e. เvingib. 1$17 / 16$ t. 


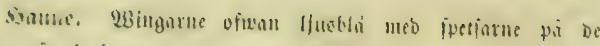
irämıre junalt brumagtiga.

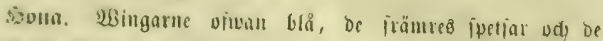
bafres framfant brebt jwartbruna; be tafre med iwarla punfter $i$ bttre fonten.

Syn. Pap. aryiolus lin. i. si. 284. 1076. Iyeena Ochs. Schm, I. 2. 27.. IV. 26. \& 144. Treitseh. X. 1.61. Vierlt. pl. 13. 1. 1. Mepliyras. Dahm. Pip. Sv. 96. 17. Irous Zett. Ins. Lapp. 912. 2. Polyommatus fool. of Hup. I.epid. I. 22:), 11. I1. quart. I. 5 pl. 11. secund. 1. 8. Ilesperia acis Falbr. E. S. III. 1. 29:̈. 124. Papilio Ilülon. 1'ap, t. 57. f. 272-274. T'oxi, 46. 11.

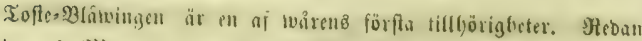

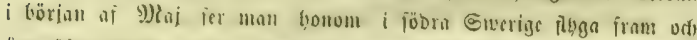

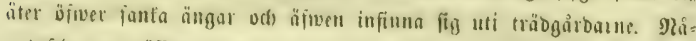
got feonare träfias lan $i$ be mederfta och nuskiga eambffipen of

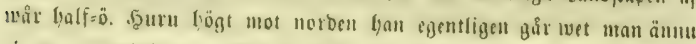

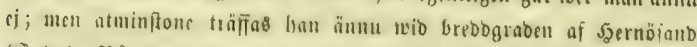

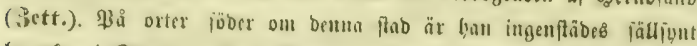

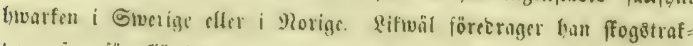
i九me framför fättlanbet.

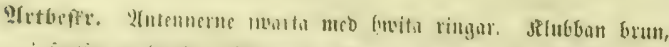
i ipetien gutagtig. Dalperme blanglint litwits, orman ods i fprto

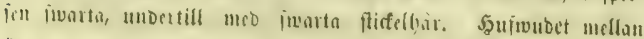

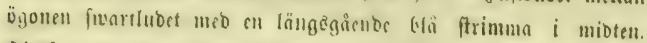

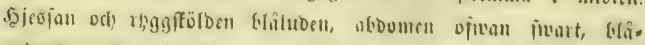

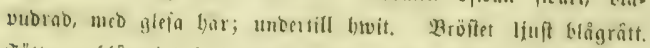
fötterne blangtight buita mod puntringlabe tarjer.

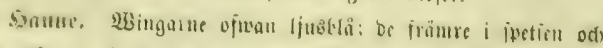

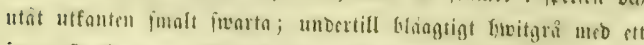

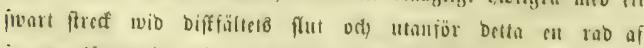

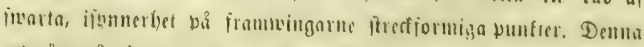
tas it pai framtringarne föga Gaggeojib, emedan enbaft fürpa munften närmaft framfonten flatr innanför be öirige. Bå bafe

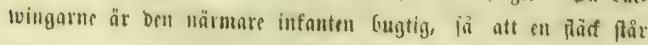


bex något inแm be ifriga, famt närmate framfanten ftarft afs bruten, fi att 2:ne kläfar fti ber wibt ffirba från be bfriga od) i linia med biffifrectet. Detta fafnas flundom alloeles, ellev finnes blott antybt. W3id bafen of bafwingarne finnas trenne fivarta punfter: en nära framfanten, en i biaffältet od en twib infans ten. Sällan fafnas enbera a bem. Stumbou finnas ợ få på Eafwingarnes unbre foba, $i$ utfanten ifynnerhet nära analförnet några otholiga firartagtiga punfter. Franjarne huvita, fii frams

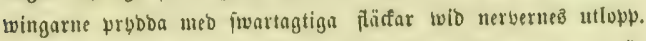
Sousan, fout till wingarnes unbre fibn lifnar batmen, är till ben öfre blå meb breb, fiwartbrum eller fiwart utfant på frammingarne ợ fromfant på bafwingarue. $\Im$ utfanten af be febnare fimes offia en rab af fumbom otybliga, fimarta fläc= far. Wingarneछ fwartbruna sller fwarta färg barierar mydet till fin utfträđơning.

Enrven, fom lefwer hå Rhamnus frangula, är gulgrön nteo

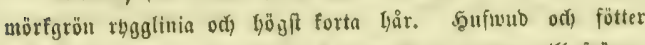
âro fwarta. \$upan ăr glatt od utan ftarpa fantor, till frânure belen grön, till ben bafre ftötanbe i frunt, famt fötfebt med en fwart rhgglinia. (Dd)i.).

\section{Trib. Brycinides. BOISD.}

Pelles 6 ; antici reliquis broviores, maris dobiles, rudimentales. Cellula discoidalis alarum semiaperfa. Costa transversa in modio abrupta, tantuin tumiditato connoxa.

Marge anticus alarum anticarum costis 3 ot ramulis duobus quartx, newpo ranulis coste ale soptime of costis $8 \ldots 10$, extenditur.

Coste alarum posticarum 8 ; 1:ma o trunco communi cum 2:da, radico simplice, egrediens ot rauulum distinctum in $10-$ bum basalom emittens; 7:ma-8:ra itorum o basi egrodientos. Costie anticarum 10, ad basin inter so equa- 
les; 1:ma o basi, T:man triramosa. cum 6:1a ex codem loco egredions.

Ala postice pro receptione abdominis canaliculate, in margine oxteriori intograe.

Jarra oblongo-ovata, depressa, luberculis carnosis hispidis ohsila, capito cordato, Tacta so conซolvit.

Papa brevis, crassa, ano mucronato, ltispislula, apice anali ot lilo per modium transverso alligata.

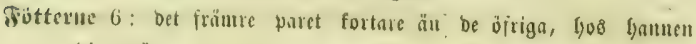
ruoimentära.

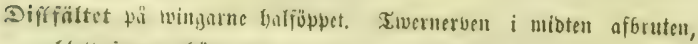
blott immmanţänganbe genom etr uppfwälning.

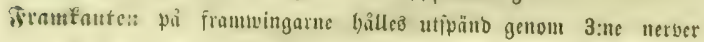
off) 2:1le grenar af en flerbe; nen!ligen genom 7:be wingner= bens grenar odi) genon 8:be-10:be singnerberne.

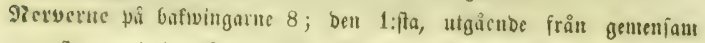
flam med ben 2:Dra, gar enfel rot vel utiänber till rotloben cn tyblig gren; $7: b c-8: b e$ utgå niterigen frủ Gajen. Fraut= wingarnes nerver 10, wio Gafen fingemellan lifformiga; Den 1:fta utgâende frîn bafen, den 7:be, fom är tregrenig, från fauma fälle font ben 6:te.

Baffuingarac $i$ inbre fanten nebböjba till en ränna, beri abbonen upptageß; i sttre fanten bela.

Qarven aflingtäggfurmig, nebtrudt, betumen mé töttiga, båriga

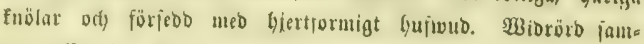
manxullar ben itg.

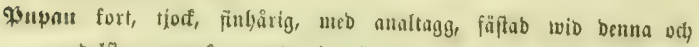
nebelit en on froppens mior flagen trio.

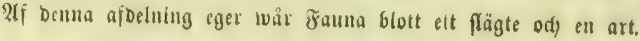

\section{Slïgtet : Ramearis. (Нйвs.)}

(Argynnis Litk. Helitata Ochs. DALs. Lycena Treitscu.

Nemeobius Sтврі. H-S.)

Antemuse longiores, clavatas. Clava dotorminata, ovata, comprossa. 


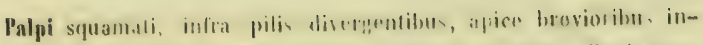
structi, porrocti, triarticulalı: articutus mrdius reliyuis conjunclis multo longior, gracitis; ultimus minulissimus omnum, osalus, asl havin articulo medio latitudino aequalis.

Ocull nudi.

Peales antici maric hrovissimi, rulimostalos tarsibus inarticulatis, fomine reliquis quidem brevioros, sed iis contormos; ft:mora sublus hirsuta; articulus primus tarsorum paris intormodii 2:do-4:to longhitudine equalis; 2:dus--3:tus invicom requales: f:tus minulu-, 5:10 duplo longior, tertio feres requalis I. eo vix longior.

llx integre.

Costa alarum posticarum 8: 1:ma of 2:di1, haes vero biramosa, c trunco communi superiore, 3:tia o rosla lransworsa, f:la -6:ta o trunco communi inforiori, ot 7:ma-8:va o basi soparatim, egrodiontes; 1:ma ramulum haud reflexum in lolum basalom omittons. Coste anticartum 10: 1:ma - hasi, 2:da-4:ta o trunco communi inferiori, s:la a) costa transvorsa, 6:1a-9:na e trunco communi superiori, ol 10;ma iterum e basi, egrodiontes; 6:La et 7:ma ox oodem loro collulie, hee triramosia. Costa Iransvorsa alarum posticarum inter costam 3:am of f:tam, of anticarum iulor 4:tam

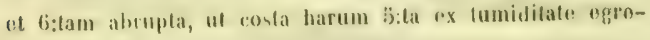
dilur.

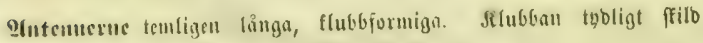

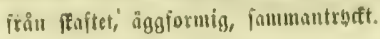

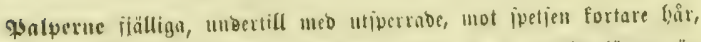
irampanende, trelebride: meblerfta lebers junal, mendet längre äl De öriga tilliammantagna; flitn feocn minft, äggrormig, wib bafen lifa breb jom dell meblerfta.

Ögonen mafna.

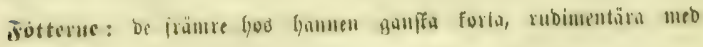




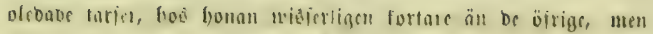
litsaal litjormign meo drsia; laten unbertill lubua; mederita pareto jörjta tarated lifa ling med ben 2:bra-4:be tillinnman; ben 2:bra vol) 3:bje ftuccmellan lifa: Den 4:be liten, bubbelt

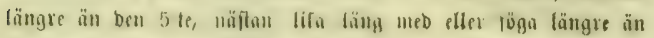
beil 3:Dle.

2zBimgneme belbräbbabe.

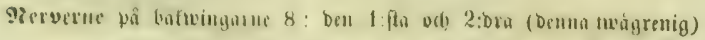

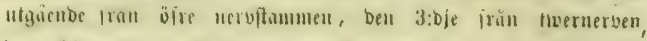
ben fibe lite man nebre nervitanment ud Den 7:be-8:De, fildo, rria balen: Den 1:ftn utiänoer en jögu bafithöjo gren "t rolloten. Ermmbingarma nervet 10 : Den $1:$ fa utgående

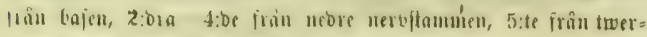

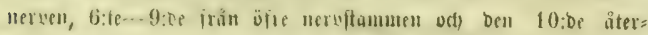

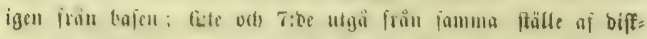
faltet, ben jebnare tregsuig. Bafmingatnes twernets aforuten melhan 3:bje ody 4:bi wingucıuen, ods iramtvingarnes emeflan

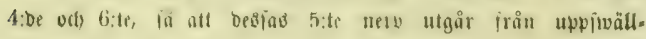
ningen.

Sgitföranbe art bar enbaft en ycueration on âret od framfon: mer om thaำ efier att baftua öfverwintrat fajom pupa.

Cralvifve-pöjoilen (Hanearis lucina lin.)

Alis supra fuscis lostareo maculalis, punctis subocollatis fuscis marginalibus; posticis infra lascis duabus macularibus albidis, 1. al. exp, 28-32 millim.

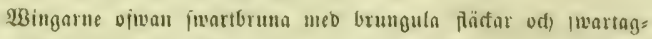
tign veclliormiga fantpunfter; De cafre unbertill ned Inenne, of

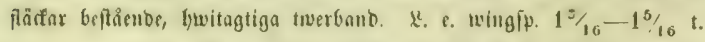
Syn. Pap. lucina Win. P.. Sv. 280. 1061. Habr. E. S. IIt. 1. 250. 778. Melltar 0ehs. Schm. J. 1. 50, IV. 14. Dalm. I'ap. Sv. 77, 10. Lyesena Treitsch. X. 1. 76. Papilio Itübn. t. 4. f. 21. 22. T'ext. 7. 11. Vemenbius II-S. Telt. I. Nickerl. Synops. d. Lepid. Fauna Bobmens. 1. 17. Lycena fierh, pl. 35. f. 6. 
(B) แm

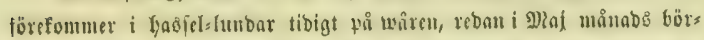
jan, Rågon annan jäfer lofal för bę̧ förefomitt, än ben Dalman uptgifaer gafwa wi äunu ej. Dmfring lipfala är nemligen arten

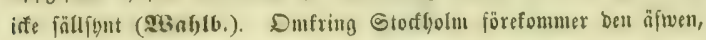
y) wilfet Dalman of torbe aifufta, bå lyan fäger: "alibi rarizes".

2/rtbeffr. 2untennerne ofwan fwartbruma med liswita ringar; tus bertifl breitagtiga. Slubban jwatt, i ytterfta fpeten röbgulagtig. \$alperne fwarta, fubna. Şufwubet med en pörre jwart, på

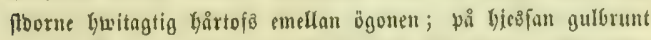
lubet. D̈gonen gulbruna, Gafait foutabe med lyitt. Nigsfföl= ben brunluben. 2rboomen ofwan brun, gleft luben; unbertill Gwitagtig. Şannens analtofö unbertill räbgul, ojman bruแ. Bröftet brunlubet. Fötterne röbgulagtiga.

Sautr. WBingarme ofwan fwartfruna (be fräute if̧n= nerbyet wio bafen) meb brungula fläđor, jom äro fmå od ptb̧bliga på baftringarne. Răngo ntfanterne gå en rab fiwart agtiga punfter, omginne ywarbers af en frungul ring, blvilfen på frammingarne är inå fỏrlängb. Unbertill äto frommingarne brumagtigt gula meb fwarta od) buritgula flädar $i$ biften, famt trefantiga flwarta flâđar längs ittanterne, motīwarnno öfre

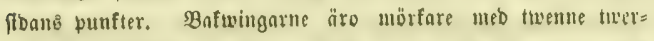

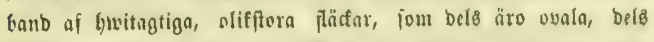
farfibiga. Rängo utfanterne få en rab fwarta, trefantiga, med

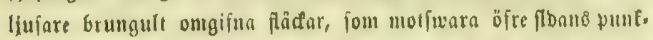
ter. Franfarme âro Knitagtiga meb fruma fläfar wio nervet= แยะ นtlopp.

Şounu är ftörre od Gar mers afrumbabe twingar än lians nen, fant någut llufare brungula fläđar.

garven, jom lefiwe pô Primula rori, odt) elatior, är

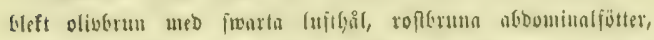

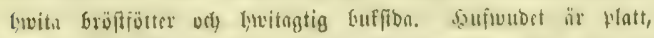
glän[andi, rofforunt, mé jivarta fibor vdy mumbelar. De phi 


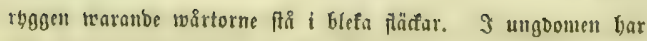
Den twenne raber iwarta borfter pả raggen, men med tilltagande silber iörjwinna beşja och lyan blifwer fläberlift bårig, famt får Våriga wårtor. \$upan är tätt öfwerwuren med fina bor= fler ody bår, yar fantig fufroubregion, imorta ivingflidor od gulugtigt hwit abbominalregion. 


\section{Fam. Hesperioidæ.}

Nargo antieus alarum anticarum ocostatus costis somper :5 simplicihus sustinetur. La apico collulie discoidalis alirum pusticarum plica subtilissima in loco costie agredilur.

(aput magnum, thoraci latitudino foro aqualc. Antmuse hasi late distantos. Stommata nulla.

Corpus respectu habito manasionis alarum erassum, robuslum.

Thorax abdomine latior, depressus, subconvexus.

Ale insecti quiescentis divaricale I. tantum antica suborecter.

Margo intermus alatum posticarum non deflesus, sed per fotam longitudinem suplicatus I. subconvolutus, ut canalis pro receptiono abdominis non lormetur.

Larna tortriciformis, fore nuda, intra folia contorta dogens. Pupa mutica, folliculata (involuta).

Framenuten af fromuingarme, fom fielf jafnar nerb, bålles nlltio utipänd medelft fem enfla nerver. Ur fpetfen of bafuingarnes bifffält utgår iftälet för nerv elt ganffa fint wedf.

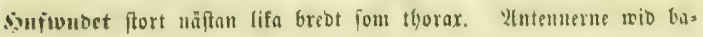
jen rribt åtffilba. §nga punfrögon.

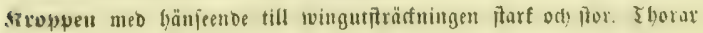
Grebare än abbomen, nebtrbrtt, föga fonver.

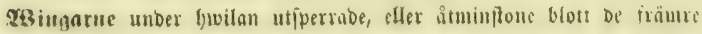

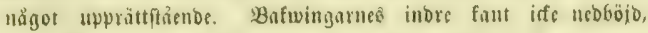

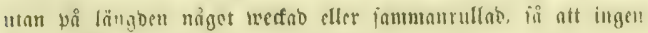
rånna för abobmens upptagande bilbas. 
Earvon, lif en WBerflarestas, näftan nafen, Iefwer bolo emellan Fammanucrflabe blad. \$upan flät, injwept i bulfa, bilbab af fammanjpunna blab.

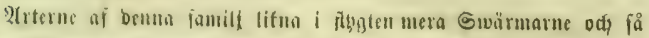
fullabe Gfyumingoijüritax, än be egentlign bagijärilarne, thuru be

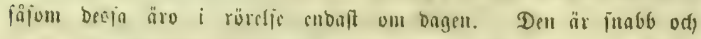

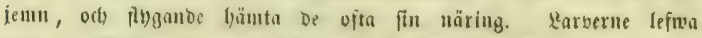

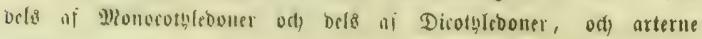

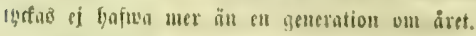

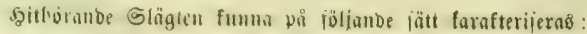

d. Tibia podum frosticorum ealearibus duobus. (Coste 7:ma el 8:va alarum onticarum approximatir).

Gifta forparets lilver löricoda mid ane porrar. I. Heteropterus.

(8tamuingarnes 7:be od) 8:be nerser wib bajen Gyaranbra näriti̊nenbe).

B. Cibia pedum posticormm ealcarihus q̨uatuor. Sifta potparets tibier nted 4:ra pporrar.

1. - Coste 7 of 8 alarum anticarum ad basiz approximatie.

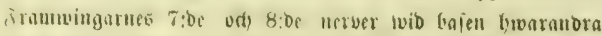
niีxthâento.

a. Costa b:La ahrum anticarum o plica transforsit cellulie ogrediens ot basi ad costam 1:tam propius accodens. Ex apice cellule discoidalis posticarum plical latud distinetat egreditur.

8ิrmuvingarnes 5:te nerv uigå fran. II. Hesperin. orffentletes twerned od fommer $i$ an. jeenbe till Gajen närmare the än 6:te wingnersen. Ut ipetien af bafwins garnes bifffät แtgå ett föga märf, bart wingwerf. 
b. Costa 5:ta slarum anticarum e plica) transversa cellulæe, sed exacte in modio inter costam 4:am ot 6:am, egrediens. Ex apice collula discoidalis posticarum plica sat distincta egreditur.

III. Syrichtus.

ชranmingatme 5:te ser勺 utgåx från biffeatltets twertwert, menl fullfomligt miot emellan 4tbe oft 6:te soingners berne. Ut fiptien af bafwingarne biffält utgår ett tybliģt mingwed̛.

2. Costæ 7-9 alarum anticarum ad basin approximatre. (Costa $5:$ ta in modio inter costam f:tam et 6:lam o plica transversa cellulæ egrediens).

Framivingarnes 7-9 nerver wib fafen

IV. Thanaos. Gwaranbra närftående ( 5 :te nerben utgår från bifffältets twerwed mibt entellan 4 :be od) 6:te wingnerberne).

\section{Sligtet : Heteropterus (Dun.) Dur.}

(Hesperit Latr, Ochs. Dan., Butlus Scop, Erynnis Scunank. Pamphila Fabr. Steropes Bossd.)

Antennæ Iongiores, clavate. Clava ovata, apice obtusa, recta.

Palpi distantes, hirsutissimi, triarticulati; articulus ultinus distinctus, gracillimus, acuminatus.

Deuli nudi.

Pelles : antici reliquis quiclom breviores, sed is conformes, tibire pedum intermediorum et posticorum ad insertionom tarsorum calcaribus duobus sat validis instructe. Articulus primus tarsorum pedum anteriorum sequento longior, secundo tertiogue longitudine requalis; secundus tortio, hic tamen quarto, quinto requante, longior. Articulus primus tarsorum 
posticorum sequentihus Iongitudine xqualis, secundus tertio hic tamen quarto, minutissimo, longior; quintus tortio fore exqualis.

llat inlegra, postorioro: aldomino breviores; antice in quieto orectas.

Costa alatum posticarum $8: 1-3$ o trunco superiore, $1-6$ e Irunco inferioro, 7-8 o basi egrediontos; cellula discoidalis ajerta, inter costas 3 ot 4 plica transversa lantum subtilissima strbctausa. Costie ularum anticarum 12: 1:ma 1) basi, 2-3 o trunco communi inforiori, 5-6 e plica subtilinsima transiersa collulie, $7-11$ o trunco communi superiori ot 12 o basi egrodientes; cosla $5: t a$ in medio intor costam 4:tam of 6:tam agrodions; sexta ot septima basi discrete; 7:ma of 8:va ad hasin approximate, ox eodem loco cellula egredientos; 8-11, inter so squaliter remota, in marginen anticum excurrunt. Cellula discoidalis abrupto truncata, aperta, plica subtilissima intor costas 4 . et 7 subclausa. Inter costam 3 et 4 alarum posticarum plica subtilissima o cellula egreditur ot in marginem posticum oxcurrit.

2/uteunerae temligen langa, iörfebda med toblig flubba. Denna n̈ggformig o(h) rả̆t med trubbig fpetsิ.

Falperue wibt aiffilba, ganffa lubna, trelebabe; ffita leben tyblig, ganffa imal, tiujpetiab.

Sgotteit nafna.

Fitterue : be iromme misferligon fortare ä be öfriga, men liffors

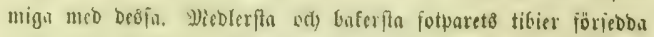
med twoune tembligen faurfa jporrar wid tarfernes lebfogning.

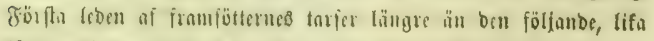
lang mob ben 2:bri od) 3:bis; ben 2:brin längre än bell 3:bic,

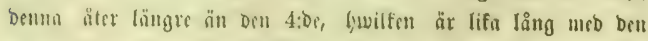
5:te. Törffa loben af Gaterita fotparets tarjer lifa ling meb be

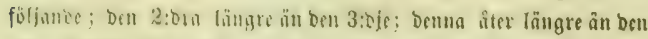
4:be, fuwilfell ât minjt; bell 5:te mäflan lita ling med ben 3:ble. 


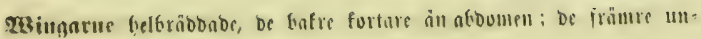
ber byilan uppåt bopflingua.

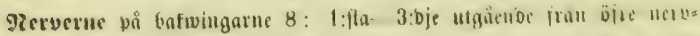
fammen, 4:be- -6:te frin nebre meluftamuen od 7:be s:be frin baisn; biffältet oppet, Wlott getnom sll ganffa fint twer=

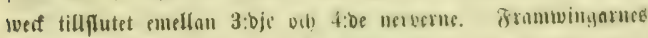
nerwer 12: 1:fta utgảenbe frăn bajen, 2:bra-3bje frîn nebre

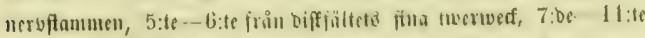
från offe neroftanumen od den 12:te frin bajen; b:te nerben

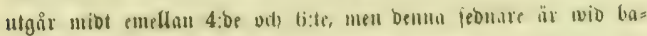
fen ffild finn ben 7:be; 7:be vily 8:be nerverme äto wid fojen

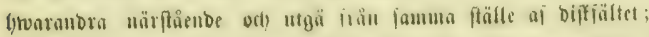

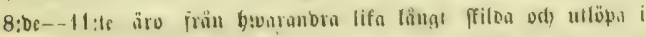

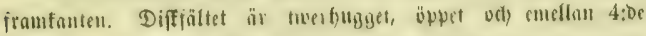

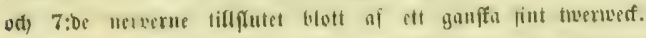
(Emeldan bafwingarnes 3:Dje od) 4:be nert ungă ur biffältet ett fint wingweef, jom löper till utfantenl.

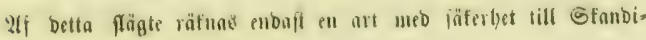

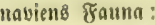

\section{Gvardfickiga tallanssmygaren}

(Heteropterus sylvius Knock.)

Alis posticis supra fuscis maculio llavis in disco serieque? minorum ad marginom posteriorem; inlat fuscis, alomis llavis adsporsis, maculis ut in patto superioro. L. al. exp. 25-30 millim.

Mas. Alis anticis suprit flavi. mieantibus, maculis nigris inequalibu, in disco, sorimpue macularum minorum ad malginem posteriorem.

Femina. Alis anticis supra furcis maculis lolvo-lulosis, fascial macnlari margin: propiore, dontala, rontinua, soriejue ad marginem posteriorem maculata, lulvis.

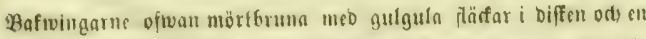

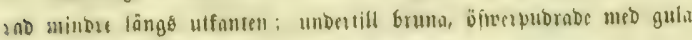




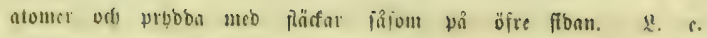
wingip. $1-1 \frac{3}{16}$ औ

Bnuแe. De häntre wingarne ofman gulogula, glänjanbe, meb otifipura, jwarts fläđar $i$ biffen od cn rab minore $i$ utfanten.

soun. De fränte ivingarne ofwan mörfbruna neb guld: gula flüfar $i$ biffen; närmare fauten ett tanbabt, jammanbản,

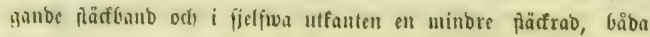
gulogula.

Syn. Pap. sylvius Knock. Beitr. 1. St. 1. 5. P. 1. z.

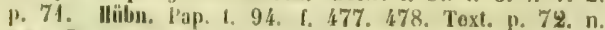
12. Pap. paniscus var, b. Habr, E. S. III. 1. 328. 242. Hesperia sylwius Mrhs. Schm. 1. 2. p. 221. Dalm. Pap. Sv. P. 201. II. 5. fied. of Inup. Lepid. II. P. 214 . 11. 27. f. 1. 2. Heteropterus. Wallengr. öfiers, af Kongl. Wot. Acad. Fürhandi. 1853. p. 22.

Demma offwer allt temligen jällünta art, lywilfen för Dalman enbaft war befant faijom tillÿranbe Finlanda gauma od beriỏre blef of bonom upptagen bland Sweriges bagfiärilar, far \$rof. S3olje:

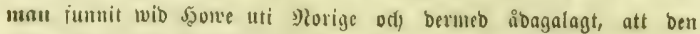
werfligen är en wair lali:ös tillhürighet. ßä uppgiina ftället träfz jabes ben oufting ben 20 Juni.

Artbefit. 2(ntemerne ofman jeartbruna med bruitagtiga lebaf= belningar, unbertifl gula. stutenn ojwan jibartbrun, i ipeticn orb) unbertifl gulbgul. Falperne gulagtigt gri, lubna, i ipetien fwarta. Jafumb ods thotur gulfrunt harige, ifonuertyet bet

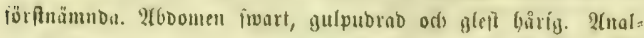
Goriten hoo lannen junrtbrunagtig, unbertill gul. Bröftet brun= grått eller gulforunt, lubet. Fö̈terne bruma, gulf-ubrabe.

Sonute. Tramtvingane viman gulgula, glänjanbe, mib

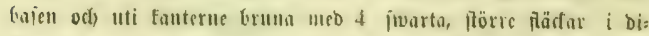
feell, od) en tab mitibre längg uttanten, al buvifa fromare of

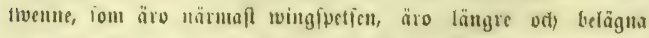

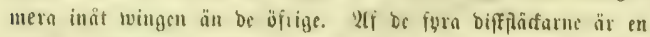
belägen $\mathrm{i}$ lielfiwa biffälltet, en wio bettas frut od be tweenue

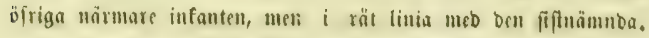


Lubertill äro frammingarne blefare gula, men för ö jrigt teftnabe fäjom på öfre fiban. Baftuingarte ofwans mörft frrma, glelt gulpubrabe, med 4-E fürre oft minbie gultgula fläror $i$ bis ffelt, od) enl rab minore buslifa längo utfanten; unbertill bruna, afmerpubrabe med gula atomer ofi) Defiutom iöricbob meb mütf=

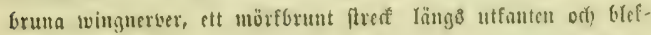
gula, mer eller minbre tholigt of frumt ombgifna guta thäfor, motjwarande offe fidons. Eranfarte fruma, mot ipetjarne Ifujare.

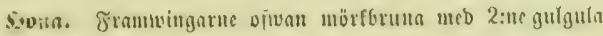

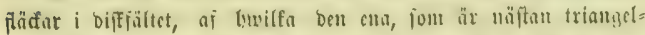

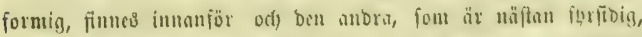

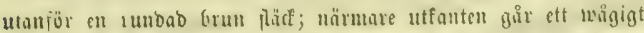
od) tanbubt twerbanto, frilonot af gulgula pläfar, af quilfa bent anbra (irân framfonten säfnabt) är belïgen närmare utfonten äı be öriģe, men lifwäl jummanlünganbe med Ecm ; i utfanten finnes en rab af fus̃, gulgula pärfar. Fürürigt lifuar fyothan bannett till färgtedningen.

2นเm. (5)

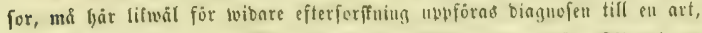

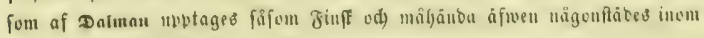
Swerige eller Morige faul tråffas:

\section{Heteropterus paniscus (lisp.)}

Alis supra nigro-fuscis, violaceo submicantihus, maculis fulvis ovatis in disco serieque macularum minutarum fulvarum marpinali ; anticis prapterea fascia maculari, dentatis, inlerrupta futwn; postiris infrafuscis, ainmis fulvis creberrime adspersis, ut in parte superiori maculatis.

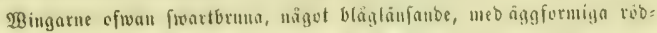
gula fläfar $i$ biffen ed en rab usinbere bylifa längs ntfanten; be fräure

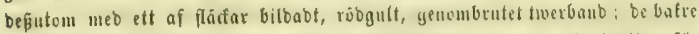

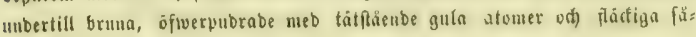
fom עึ ôjre fiball.

Syn. Pap, paniscus ESP. Selm, 1. t. 28. Suppl, 4. f. 2. p. 322. 1. 105. c. 60. 1. 6. P. 14, rar. FABR, E. S. III. 1. 328. 242. ilespenta Ocus. Sehm. 1. 2. 249. IV. 33. Treitsch. X. 1. 93. Dals. Pap. Sr. p. 201. 4. God. et. DUP. Lepid. I. p. 231. pl. 12. f. 1. 2. Pap. brontes HoBs. Pap, t. 94. f. 475. 476. 


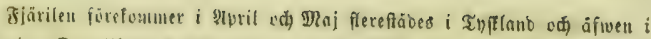

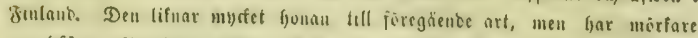
grumbfärą, röbgulare fä̉far od) bet rötgula twerbanbet, fom bå frammins

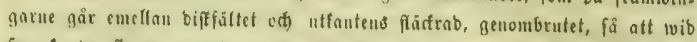

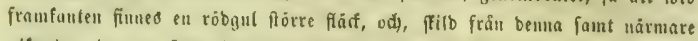
uffonte", twenne fmu, ftundom otubliga, famt berefter $i$ tät linia meb beu

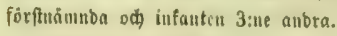

\section{Slägtet : Hesperian (Latr. Ochs. Dасм.) Boisn.}

(Ileteropterus Duмenu. Buttus Scop. Erynnis Scurn.⿻一, Pamphila $\mathrm{FABR}_{\mathrm{AB}}$ )

Antenna longioros, clavatie. Cliva elongalo-ovata, recta, apico intertum mucrone hamiformi reflexo munito.

Palpi distantes, hirsutissimi, triarliculati; articulus medius maximus, latus, ad insorlionem articuli basalis latior, inflatus; articulus basalis latimdine modio fore iequalis, sed ultimo longitudino haud requans, intordum oo multo minor; articulus ultimus gracilis, cylindricus, subacuminatus, foro nudus, prope marginem apiculom superiorem articuli medii insertus, basali longior, interdum medio longitudino hand codens, in pilositate articulorum reliquorum non occultus.

\section{Deuli nudi.}

Pelles: antici reliquis quidem breviores, sed is conformes, Tihie pedum intormodiorum ad insertionom tarsorum calcaribus duobus ralidis munite, sed tibie postoriorum præterea halient calearia duo hreviora fere in medio. Femora infra hirsuta. Articulorum tarsorum proportio fore ut in genere præcodente.

Ala integra, posticie ahdomino haud breviores; antica in quioto oroctix.

Costr alarum ut in genere antecedento; costa f:ma sou truncls communis suporior alarum posticarum ramulum rectum, apico erga corpus insecti spectanlem, in lobum basalem omittit; costa bita alarum anticarum, quie sola o plica 
Ionuissima transvorsa rellula egredit, haci ad roblam 4:1anm propius accedit; costa bita non o plica transversa, sed e Irunco communi superiore oritur. Collula discoidalis alarum anticarum ost oblique truncata, aporta, plica tantum subtilissima clausa. Li plica transversa collula discoidalis posticarum plica subtilissima land distincla intor rostas 3 ot 4 egreditur.

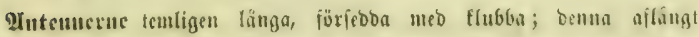
äggformig, răt, i fpetjen unberfunbom föriebd meb cu frofior. mig, tillbafabólo taģs.

\$alperue wibt itffilon, gauffi lubna, trelebabe: meblerfta lioen gunfta ftor, breb, wio Gablebests leojugnisg frebare, uppolait; basteben näfan lifa bred med ber meblerftn, men fuapt läнgre än Den ffla, unberftumbom musfet minbore, än benus; fiffa lébcu

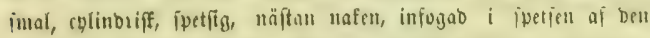

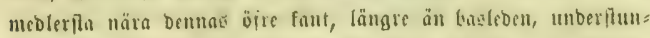

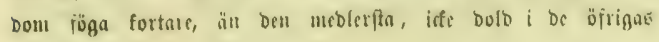
bårigbet.

S̈goueu nafna.

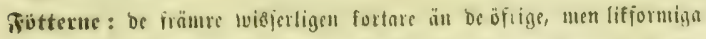

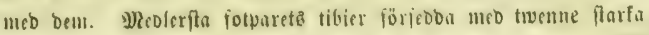
iporrar wio tareleost, men baferfita pareto tibies bafiva dézutum

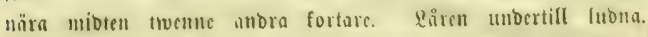

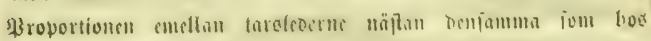
jơrc'gåcnde fägre.

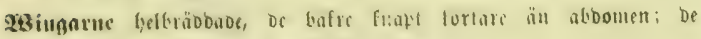
¡rỏmuc unbet brvilan uppât bopflagne.

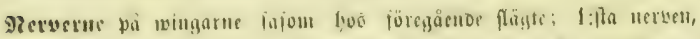

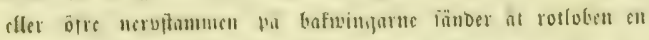
rät gren, fom mó jpetien är sigtab cntor injeftent fropp; 5:te

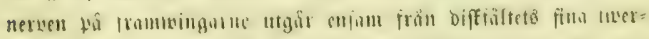
Ived od) fonmes i anjernbe till laien närmate 4:De än fi:te

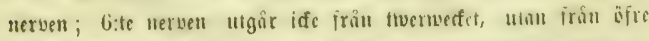

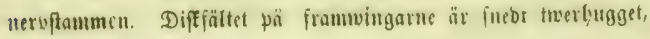


öppet, Glott gerrour ett ganffa fint, twertvect tillflutet. Frän biffẹaltets twerwecf pa bafmingarne utgår ett ganffa fint, iöga märt6art tvec emellan 3:ble och 4:be nerverne.

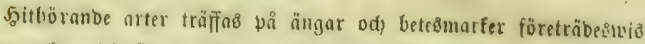
$i$ fobra od mederfta Iandfaperne of Standinavifa lyalf=ön. Teras

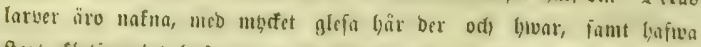
flort, flotiormigt buftud ofb föfth froppertingen jmalare än be ofriga. \$utpan är foniff ody imärt, famt flutar frautill med ell tajge od an vå luffifon füritoo mó en förlängo fliba, fom innefluter fpiralum: gan. I anjectibe till Demna fliba lifuar ben pupan till stattijüril=

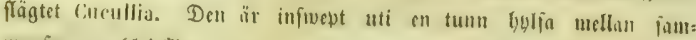
manfpunna blab*).

\section{A. Antemulinbloan i spetsen trubbig, utan tagge. Pal- pernes sista led naistan lika lang med den medlersta, myeliet längre in basleden.}

\section{1. himdre 'inceismygaren (Hesporia linoola Seriba.)}

Alis snpra fulvis, anticis maris lineola nigra tenerrim:? in disco femine immaculatis; posticis inlra griseo-flavidis, margino interiore Havidn; clara atennarum intra apice nigersina, L. al. $\exp .25-30$ millim.

Wringarne olman röbogula, be främre bos baunen med en fin, fwart linia i bifen, men hos butan opläfabe; be Gafre unbertill grågula, $i$ inbre fanten gulagriga; antemnflubban unbertill $i$ ipotien fiwart. \&. e. waingfp. $1-1 \frac{3}{16}$ t.

") Tabula subdivisionum hujus generis:

A. Clava antennarum apice obtusn, non hamato. Articulus palporum ultimus unedio longitudine fere aqualis, hasali multo longior: II lineola $\$$ linea.

13. Clava antennarum apice hamalo. Articulus palporum ultiutus tnedio multo brevior, basali haud lonpior: $I /$. comma \$ sylvanus. 
Syn. Pap. lineole Seriba, Journal, III. St, p. 244. Ilesperia 0chs. Setim. I. 2, 230. IV. $34 \&$ 161. Moist. Icon. p. 243, pl. 47. 1, 4, 5. Pap. viryula llïbu. Рар. І. 130. Ґ. 660-663. Hesp. lineola Wallengr. Kongsl. Wot. Acarl. Ofversigt 1853. p. 23. n. 1.

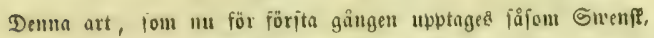

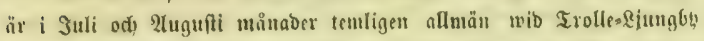

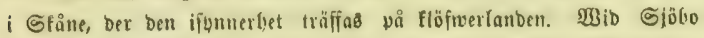

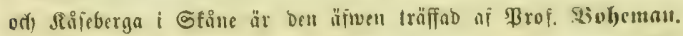

QIrtbeffr. 2(ntenterne ofwan röbgula, unbertill gulantigu. Rlub=

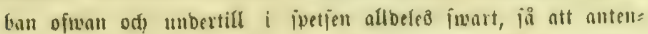
nenв gula färg endaft på cna floan ué en imal fpets utlöper vâ flubtan. ظalperne gulagtiga med röbgula od brunagtiga,

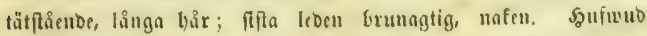

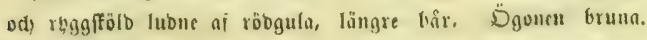

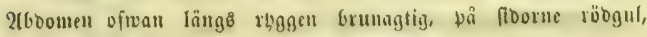
unbertill ljujare; analforften ljuft röbgul. Bröftet brvitgult, libbet. Fỏtterne röbgula.

samuc. WBingarne vfonn wacfert rängula med mbfet

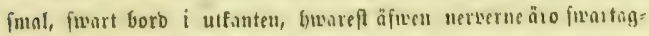

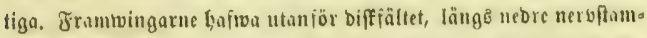
men en fin, iwart linis, jom år alltid rät, men ftundom fnfnaถ ben eller fiunes blott oţblig. Franjarne äro gulagtiga. Un= bertill äro framuingarme $\mathrm{i}$ biffen röbgula, mot ifetfarme lfuछs guin ofl) $i$ inbre fanten wib cafen fwartagtign. De bufte äto pa famma fion ljuft guta meb fung grangtig anffroftuing ods i inbre fonten gulagtipa, quilfen febnure färg utan beflïmo

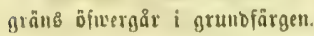

Soman lifuar till färgen bamen, men foftuar ben fwarta

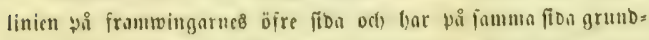
färgen mågot buftrare, od ftunbom brunggtigt anlupun bafuingar.

\section{Gijue "liatelsmygaren (Ilosperia linea W. V.)}

Alis supra fulvis, anticis maris linea nigra in diceo, femine immarulatis; posticis infra flavo-griseis: macula llava ad 
marginem interiorem; clava antennarum infra apico fulvo. I. al. oxp. 33 millim.

WBingarne ofwan röbgula; be frümre yos bannen meb en fiwart linia $i$ biffen, gos bonan ofläfabe; be bafre unbertill gulagtigt grå meb en böggul plär länge inbre fauten; antennflubban i fpetien une bertill röogut. \&. e. mingip. $1 \frac{5}{16} \mathrm{t}$.

Syn. Pap. linea W. V. p. 160. Fam. A. n, 5. llïbn. Toxt. p. 72. n. 16. pl. 96. 1. 485-487. Hesperia Mabr. I. S. III. 1. 326. 236. Oclis. Selim, I. 2. 228. IV. 34. Treitseh. $X, 1$. 97. Dalm. Pap. Sv. p. 200, n. 1. liod. et Dup. Lepid. I. p. 233. pl. 12. tert. 1. 2. Pan. Iluenmas Esp. Schm. I. t. 36. Suppl. 12. 1. 2. 3. p. 344. Pap. renula lliibn. P'ap. f. 131. f. $666-669$. llesp. linen Walleugr. K. Wet. Aead. Ofv. 1853. p. 23, n. 2. Denna fiäril*) är, âtminfone enligt min exfarentyet, päliphntare än föregåenbe. Den förefonmer nib famma tio, fom benna. 2tf Dalman angiimes de öftra probinferna of Swerige, od ferffilot skefinge, låjom wiffelicort. Srån Gfåne bar jag fett ett exemplar, tagit af ßrof. Sutberuall.

Artbeffr. Större Tåtelīutgaren lifnar fả ben föregåenbe, at bet är tiffrädligt luär endaft angifma ffiljagtiglyeterne. 2(ntenn. flutban är unbertill inart med röbgul ipet8. Frantringarme

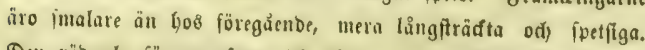
Den röbgula färgen ofiwan ide få ren ody wacfer. Den juarta borban pio ifre fiban af mingnrnes uffanter brebare od tvinge nerverme, ifunnerbet hos lyonan, näptan till bela beras längb fwarta. Framusingarne äro unbertill i fpetisn grå meb gul an= farbfning od) bafiringarne âro på famma foba mörfare grå od)

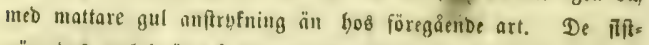
nämmba rotlob är tå jamma fiba gulagtig od $i$ infanten fins.

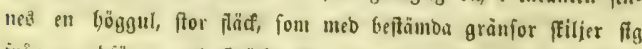
frîn grunbiärgen od fräder fỉ̧ filformigt frin utfanten năftan

") Hesperia lineola est in Succia meridionali, ". gr in Scania, sat frequens, sed II. linea rarissime occurit, lantum a Celeberr. Pof. Sundewall in Scania cl Dalmann in Blekingia capls. 
änoa till mingbafen. Şannen bar $i$ biffen of frombungarne

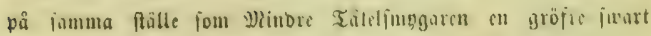

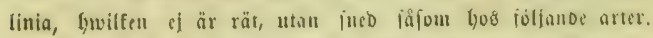

Sonven är mörfgrün med en mörfare rugghlinia, od tureme quita foolinier, fruilfa äro fantabe med fwart. Den lefuer ba

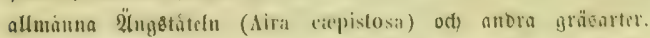

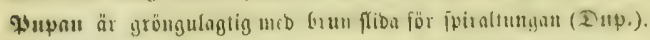

\section{Antennhlubban i spetsen försedd med en liroli-} formigt tillbaliabojjal tagg. Palpernes sista led mychert kortare än den medlerstil, hnapt längre än baskedem. 3. Allmänna Ängsanygaren (Hosperia comma lin.) Alis posticis inlra favido-virescentifus maculis quadralis albis. L. al, exp. 25030 millim.

Mas. Alis supra fulvis marginibus infuscatis, maculis quadratis favis ohsolelioribus, ot in disco anticarum linma obliqua nigra, argenteo inscripta.

Femina. Alis supra fulvis fusco inductis maculis quadratis flavis distinctioribu:

Bafningarme undertill gulagtigg gröna med furfibigu, brvita flïfar. 2. S. wingip. $1-1 / 3 / 16$ t.

5yanue, Wingarne ofwan röogula med trumagtiga utfan* ter, otgbligare, farribiga, gula pätar ods i biffen ai rrun= wingarne en jucb, fwast linia, jom $\mathrm{i}$ mioten âr teftuo moro en amnan, glänfanbe flffwerfärgab.

Souna. SBingarne oiman rübguta, örweroragna med butut

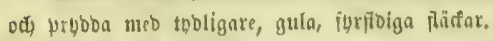

Syu. Pap. comma Lin. F. Sv. 285. 1080. Iläbn. Pap. t. 95. 1. $479-481$. Toxt. 72. 13. Hesperia Vabr. E. S. III. 1. 325. 233, 0chs, Sclom. I. 2. 224. IV. 34. I)alm. P’ap. S\%. 201, 3. Lett. Ins. Lapp. 915. 3. Hod. ot Dup. Lepid. I. p. 237. pl. 12. tert. f. 4. II-S. Toxt. 1. 159. Wallengr. Kongl. Wot. Acad, ofvorigt 1853. p. 23. n. 3.

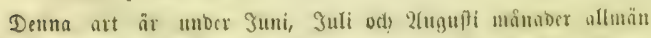
på Yyela Gfanbinaviffa balfeön ăbo upp i Lappmarfon od) ơinmar- 


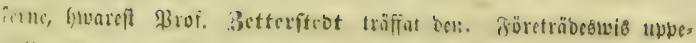

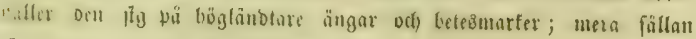
förefonmer ben på fỉblänbta fällen.

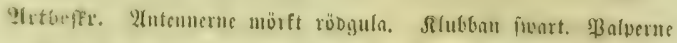

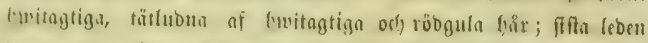

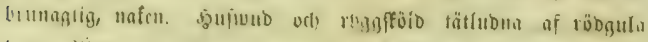

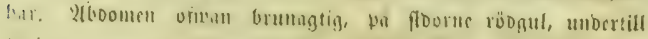

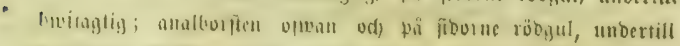

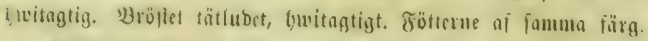

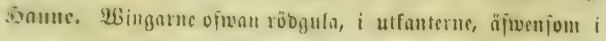
tafuingarnes framfant, funat furabruna; be främre $i$ wing=

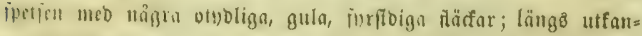

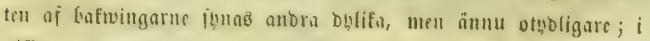
Diffen af frommingarne at grof upwlöfo, imart linia, jom läng

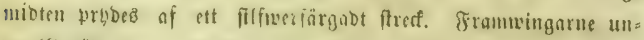
Dertifl röbsuln, $i$ jpetfen grönagtiga, wio bafen funartagtiga off med antşoning till öfre froms fwarta difflinia; Färfarme i wing.

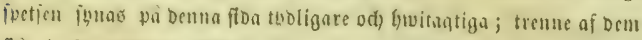
flì i framfanten, frtba frîn lywaranbra genum nerverne, ods

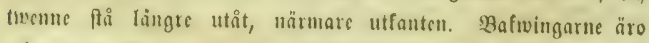
Whi unbre froan gulagtiga, farft öfwerbrigna med grönt od)

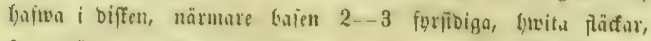

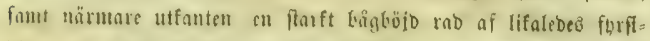

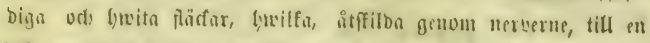

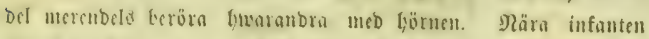

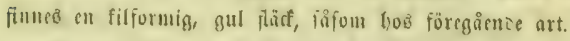

souran lifnar till färgeffuingen fannen, men fafnux ben

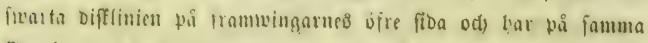
Tiba freonre frum losb i mingarmes utfanter; orfutom är ben röbijula grunbfargen öpwerbragen meb brunagtigt, fa att win= garmes örte flor ftöter mera $i$ eruent. Demua bar offio flera

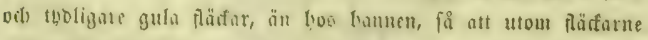
i framusingarnes fpetfar od läng8 Gafwingarne utfanter finnas

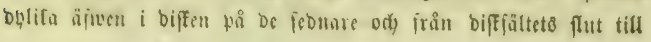




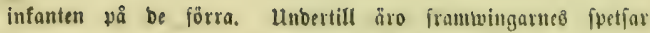

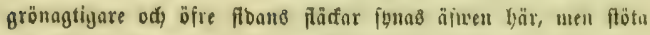

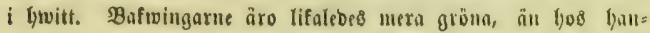
uen, od jafna ftunbom lâađar i biffen.

Sacven är jmutfigt grön, ftötande i roftorunt med col rib

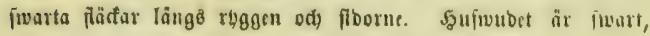
omgijwet af ell bwit, fwartfantab ring. (Sch)f. Dup.).

\section{Storre Ẍngsmygaren (Hesporia sylvanus Esp.)}

Atis posticis infra flavis, haud virescentilus, maculis quadratis flavis valdo obsoletis. L. al. oxp. 30-33 millim.

Mas. Alis supra fulvis marginibus lato infuscatis, maculis quadratis flavis obsolotioribus of in disco anticartum linea obliqua nigra.

Femina. Alis supra fulvis, fusco induetis, maculis quidJratis flavis distinctioribus.

B.afwingarne unbertill gula, iögn fiötanbe $i$ grönt, ueb fyrfibign, gula, ganffa otboliga fläffar. \&. e. เwingip. $1 \frac{3}{16}-1 \frac{5}{16}$ t.

5anue. Woingarme ofwan röbgula med breb, brun bord $i$

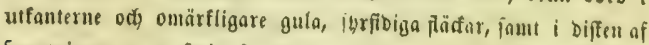
framivingarne en fueb, fwart linia.

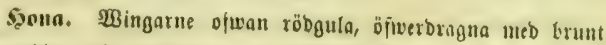
ody probba mé tybligare, gula, furfibiga fläfar.

Syn. Pap. sylvanus Esp. Schı. 1. 1. 36. Suppl. 19. l. 1. p. 343. llïbn. Pap. t. 95. I. 482-484. Text. 72. 14. llesperia Fabr. E. S. III. 1. 326. 237. Mchs. Schim. I. 2. 226. IV. 34. Balm. P. S'. 201. 2. Goul. ot Dup. Lepid. I. $235 \mathrm{pl}$. 12. socund. I. 2. II-S. Text.

1. p. 159. Walleugr. Kongl. Wot. Acad. Ofrersigt 1853. p. 23, 11. 4 .

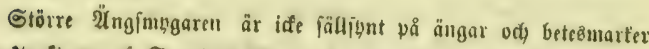
i ffogetrafterne af Siveriges föbra ody mebletifa lantofiaper äfweniom

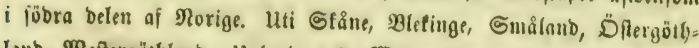

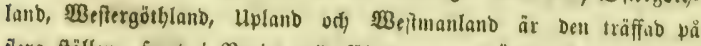
flera ftäLlen, fant i Norige wib Elyriftiania, i D̈fterbalen od) (S)ubs

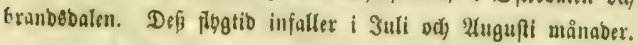




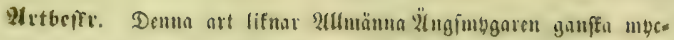
fet od) är lätt aft förblanba bermed. Den ffilies bod berifrän

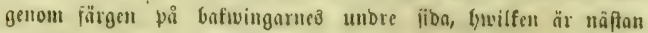

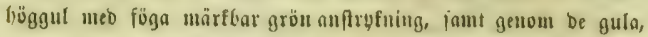

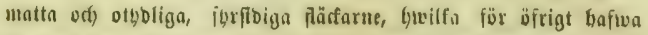
bibe på baftuingar od) framuingar famma ftälning fom hoğ nämune art. Dire fitoan är befutom mörfare meb brebare fimart. brun borb $i$ utfanterne of wingarne ofy bannens biffitref faf $=$ nar lyelt od) bållet ben filfuerbwita linien, fom finneô $\mathrm{i}$ mioten Deraf lyos föregående art. Eärgftilnaben fönen emellan ät ben=

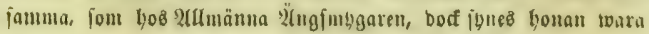
mörfare på wingarnesి öfre fiba än hos benna.

\section{Slïgtet: Syrichtus Boiss.}

(Ilesperin Ltти. Ocus. Dам. Zетт. Heleroplerus Dis. Buttus Scop. Erynnis Scutaxi. Pamphila Faвr. Thymele Fan. Stemi. Syrichlus el Spilothyrus Dor.)

Antenna longiores, clavatxe. Clava clongata, sensim incrassata, obtusa, nonihil arcuata.

Palpi distantes, hirsutissimi, triarticulati : articulus medius masimes, reliquis conjunctis major ovaluc, ad inserlionem articuli basali gracilior, apicem versus latior, tumidus; articulus ultimus in apice medii insertus, gracilis, ovatus, basali tongitudine fore equalis, subnudus, in pilositate reliquotum foro occultus; articulus basalis ultimo nonibil brovior vol oo longitudine el articulo medio latitudino xqualis.

Pelles ut in genero prioro; sic otiam forma tibiarum; sed proportio articulorum tarsorum alia est: arliculus primus pedum anteriorum soquentibus conjunctis aqualis, secundus tertio, hie vorum quarto, minutissimo, longior, quintus tertio auualis; arliculus primus pedum posteriorum sequentibus longitudine aqualic, secundus tertio, hic tamen quarto longior, guarlus quinto xqualis. 
Ala intogre, postice longitudinom abdominis non superantes; omnes in quioto horizontatiter domissa.

C'ustie alarum it in genere precodonto, sod ramulus cosle primat posticarum sat brovis, of costa b:ta anticarum, cum costa 6:La e plica transversa collulie oriens, exacto in modio inInr costam f:1an of 6:tam rgredifur. Intervallum basalo inter costam 8:vam 91 9:nam anticarum multo minus, quam intor costam 9: $n$ am of 10:mam. Callula discoidatio al.1rmm anticar um est abrupto truncatia, aperla, plica tantum tenui,sima clausa. E plica transvorsa collula discoidalis posticarum plica subtilis, otsi sal distinch, inter cosłam 3:am ot 4:tam loco coste ogreditur.

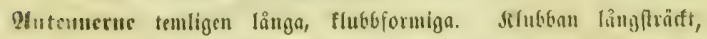
fmåningou tilltioffnanbe, trubbig, något böio.

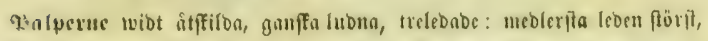
ftörre än De öriga tillfammantngna, äggformig, twio lediogntin Ben med baeteden imalare, mot fiperfen brebars, jwilld fifta le= Den imfogad $i$ ipetien af ben medlerfa, imal, äggformig, mäftum lifu lång meb basleben, näfan unfen od bolo $i$ be öfrigas bå vightet; Gaaleben något fortare än, eller lifa lang ued den firta, odi) lifa breb meb ben meblerfta.

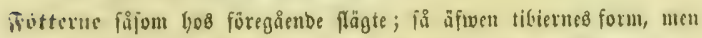
fürlyålanbet mollan tursleberne är annorlunba: 1:fta leoen af irümfta fotparets tarjer lifa läng med be jüljonbe tillfammans tugna, 2:bra längre än 3:bje, benna längre än 4:be, f)wiffen ä gauffa liten, 5:te lifa ling med bell 3:Die; 1:fta leben af bufre rotparetis tarjer lifa linn med alla be följanbe tilljammantugna, 2:bra långre ån 3:Dle, bennก läugre än 4:be, f)wilfen är lifa ling meb ben 5:te.

2:3iugarue helfräbbabe, be bafre lafe g̈fwerftiganbe abbomens längb ; alla unber Guilan Jorizontelt nebflagne.

Sictverue på tvingarue fâfom hoo föregående flägte, men 1:fta nel: venb på latroingarne gren gamfea fort od) fraumwingarnes 5 :te nert, fon tillita med ben 6:te appfomuer frả biffjältets twers 
Ivert, utgair fullfomligt mibr emellan 4:be or(j) 6:te wingnerwerne.

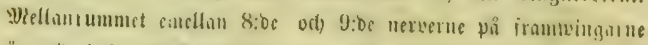

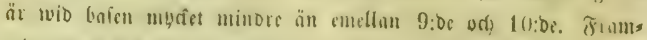
เvingnance biffin̈lt är twerlugget, öpwet, endaft tillflutet af ett

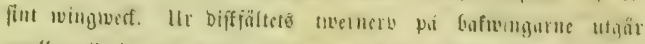

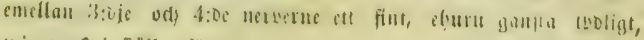
wingwert $i$ fället for nerv.

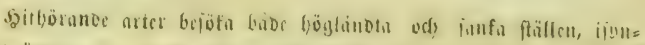

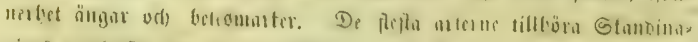

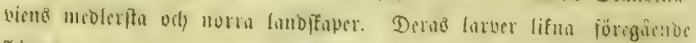
fligtes, men puporne äro fonijfir och furifa cmeflan fammanjpmun blab uti en glegి bulia *).

\section{A. De livita flïeliame emellan I:sta och 2:dra ner-} verne pai balivingarnes undre sida nistan lika stopa, (den ytte iche storre ant den vid basen).

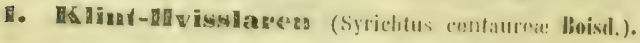

Alis cupia ob curo fucei, in nigredinem vortonlitus, ma-

*) Tabula suhdivisionum hujus generis:

A. Hacula alba inter costam 1:mam et 2:dam in latere inferiori alarum posticarum fere aquales (postica basoli non major) : S. ceniaurea.

13. Maculo albe inter costam 1:man et 2:dam in latere inferiori alarum posticarum inæỵuales, (postica hasali eridenter major).

I. Maculeo albo, ad fasciam in latere inferiori alarum posticarum pertiuentes, inter costan 3:tiam of 4:tam subequales, intus abrupte truacate: $S$. alveus of serratula.

2. Macula albe, ad fasciam in latere inferiori alarum posticarum pertinentes, inler costam 3:tian et f:tam inrquales, antiea longior, versus basin ant versus marginem posteriarem prominens: S. andromede of malve. 
culis quadratis albis, posticarum obsolotioribus; anticis sublus fuscis, cinereo obductis, maculis albis ut in parte superior" lineisque longitudinalibus inter limbum albidum ot soriom macularum; posticis subtus nigricantibus, haud viresconlibus, costis albidis, maculis basalibus albis, sorio macularum albidarum continua suargineque interioro nigro-cinereo; clava antennarum antico obscuro brunnea. L. al. exp. $32-33$ millim.

Wingarne ofwan mörfbruna, flötande $i$ fwart, med hivita, furs fibiga fả̆ar, fou wå be Gafte äro otybligare; be fränte unbertill

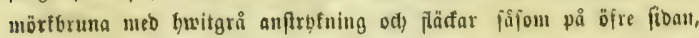

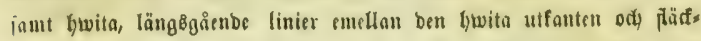
raben; be Gafre unbertill fwartagtign, föga fötanbe i grönt, meb b)vita nerver, bwita fläfar srib bafen, \&n fammanlyänganbe rab af anbra bดlifa, od fmartgrå infant; antennffuttian i främre belen mòrft Grun. \&. \&, ivingiv. $1 \frac{5}{16}-1 \frac{3}{8}$ t.

Syn. Syrichtus centaurea Boist. Hesperia II-S. Fost. I. p. 15\%̆. pl. 1. f. 1-3. Hesperia frifullum Dalm. P. Sv. p. 202. 7. (cortissime. Alii in Musno ojus non exstant.) Zett. Ins. I.app. 914. 1. ox. parto. Ilesp. alocus God. of Bup. Lepid. Suppl. I. 259. p1. 42. 1. :. 4.? (excl. synon.). S. centaurea Wallengr. Kongl. Wot icad. ofversigt ious: n. 24, n. 1.

obs. M. carthami Hïbn. valdo affinis sed re ver'a distincta. Magnitudo minor; macula albe alarum. posticarum in parte superiore obsoletiores (series. interna non tam explessa quam in illa). Macula interna inter costam primam et socumdam in parto superioro alarun anticarum dupplex, ubi illa maculan albam geret unicam. Nle postica eliam infra. obscuriores sunt, quam in illa, haud virescentes, ot costx albæ, minimo virescentes.

Denna art tillyör enbaft ben bögre norben of wår balf 30 , ber

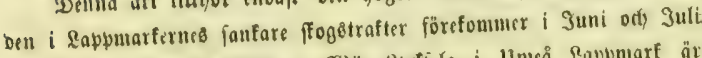

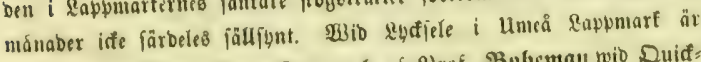
Dell tagen af \$roj. Betterifteot, od) af \$rof. Bubcman wio Quid

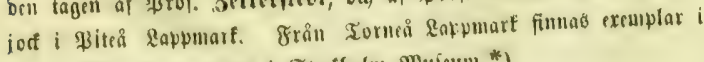
Rongl. Wet. Ucabeniens i Etodfyolm Nujeum *).

-) S. centauree Labitat in Lapponia: ad Lycksele, Quickjock et l'ornee. 
2lrtbeffr. 2Antennerne opwan fwarta meb Glwita ringar, unbertill b) witagtiga. Rlubban ofwan jwart, framtifl brun eller mörft röbbrun, umbertill od) Gnftill fywitgulagtig. Balperne tätlubna, Gixita, med inglanbabe mörforuna viar; iffa leben fwartbrun. Şufixub od) ruggftölo tät grålubua. 2(6bomen ofman imart med grá lubb, unbertifl bwitagtig. 2(nalforften till öire bälften Grunagtig, till ben unore fywitngtig. Bröptet fartt lubet, mörfs grůtt. Fötterne mörfgră mé gulagtiga tarier.

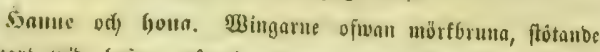
$i$ fwart, wib bajen gråagtigt lubna. Frannwingarne främre

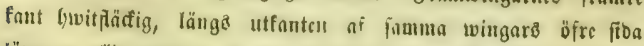
löpex en flingrande rad af f̧urfantiga blwita pläfar, af hyvilfa bell trebje fro̊n infanten od be trenue närmaft iramfanten was

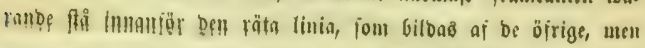
lifuäl iffe i linia meb Guwarambra; $i$ bifffältet en förre bnvit

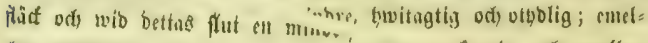

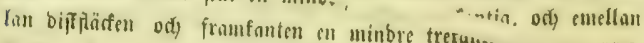

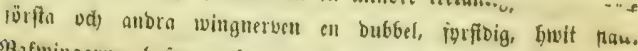
Bafwingarne lajwa ofwan wio bajen en sller amnan othblig, (1) witagtig flăd, $i$ mioten of fromfanten en ftor, sent gwit ods irån bema est twerbuno af andra otnoligate, fant bejutom nära utfunten en rab minore, olifformiga fâffar, ala hwitags tiga, shuru en od) amunu, fumbon be fiffa funas lifiom faus

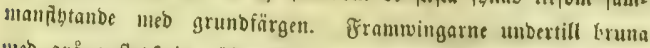
utro gri̊ anftrbfning öfwer bela btan, famt äro, bwad be lowita fläcfarme beträfîfar, tedunoe fåjom på öfre fiban, med unonutag beraf att Den bublla päces emellan 1 : pipa od) 2:bra nerven bär

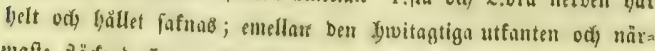

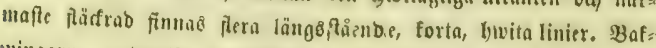
wingarne undertill fiwartagtiga med ofetḩblig, grönagtig att=

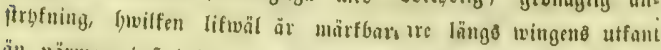

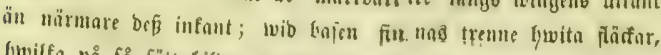
Grwilfa på få fätt birba en twerrab, at be thenne inbre berobro

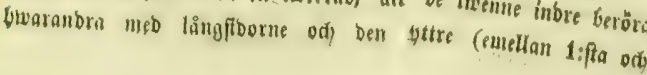




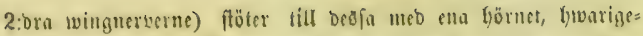
num ben fonumer med bela fin längo frafum de Dirign; öfwet

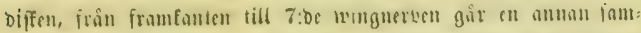

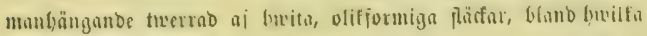
ben cmeflan 1:fta ods 2:bra wingnecuen warmoe är lifa por,

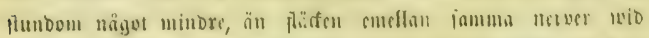

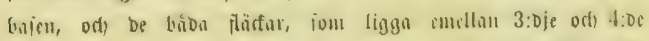

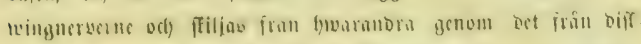

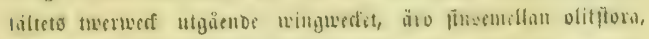

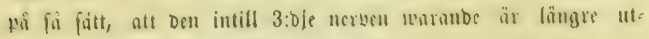

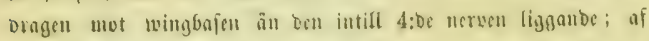

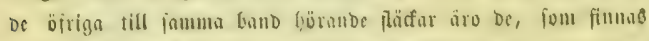

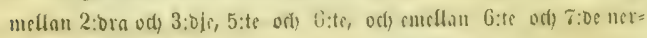
werne, trefantign, men ben emeftan 7:oc vd) s:oe wimmerwerne

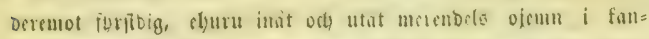

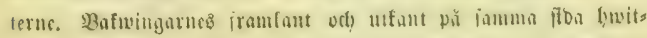
agtigs, ten iednare med en jerie furisalabicirfar, iom begränja

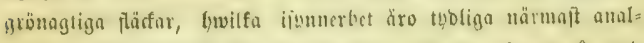
fiörnet; wingnewerne äro rent buvita; infanten fiwartgrif med

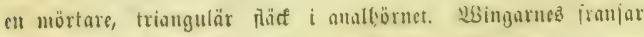
äro ofwan od) ambertill furita med bruma fliffar ber nerwerne utlōpa.

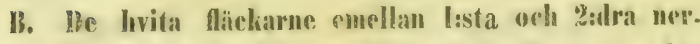
verue pâ bahvingarnes undre sida olilistora, (den yttre större in den vill basron).

1. De heilu flucliarne emellen 3:dje och hide vingnerven, heillin villhora loerbandet po balivingarnes undre sidu, nëstan liku stora, inat tvert afhuggna.

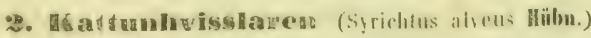

Alis supra obecure fuscis, in nigredinem vertentibus, cinereo ubobducti-, matulis quad ati: allis majoribus, postaram

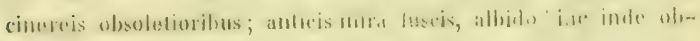




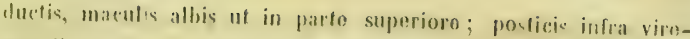
serblibur, costis roncoloribas, maculis basalibus fasciagure discoidati maculala ronlinua albis, ot margino inlerno cinoroo ma-

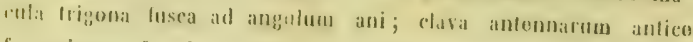
forruginoa, L. al. exp. $27-30$ millim.

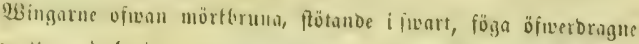

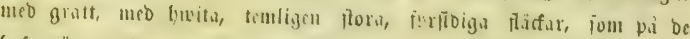
Lafie äro glabatiga ofe ofwoligare; de främre undertill bruna meo

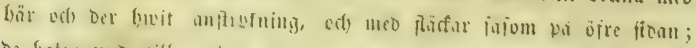

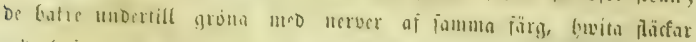

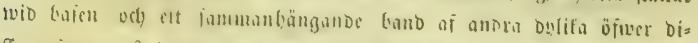

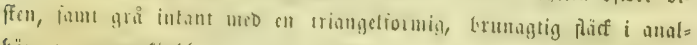

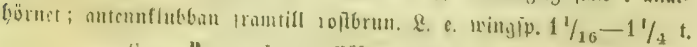

Syn. Pan. alneus llähn. Рар. pl. 92. f. 161-4.63.

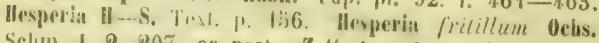
Selui,. 1. 2. 207. ex parto. Zett. Ins, Lapp. 914, n. 1. ox partu (cum Syrichlo centaurea et serraiule com-

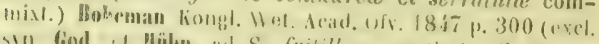

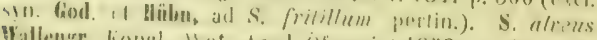

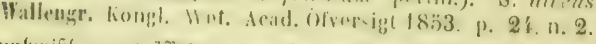

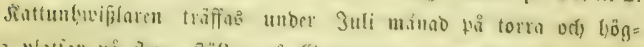

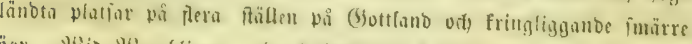

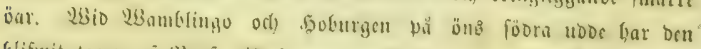

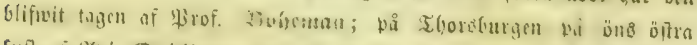

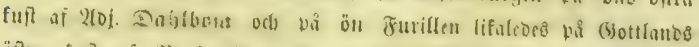

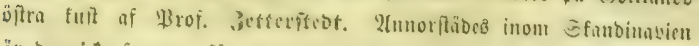

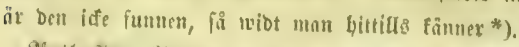

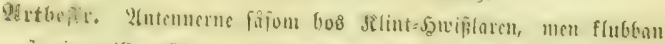

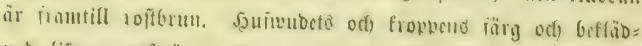

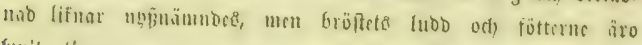
guvitagtigare.

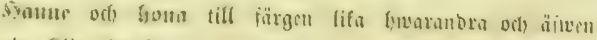

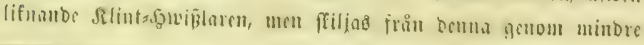

") S. aleens hahitat in fontandia ad Wamblingn. Hoburgen et thoosburgen, ot in instula Furillen. 


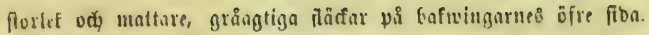

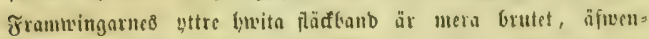
fom läfen $i$ famma เvingars biffiâlt är minore. Till tringar= nes unbre fto lifnar benua fjäril äfwen nämmbe art, men fram=

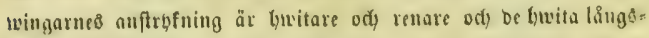
fircfen emellan famma uningar ljufare utfont ody Guvita plärfab

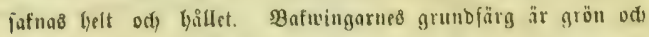
wingnerverne liafiva fanma gröna färg. Defiutom är bi liaf:

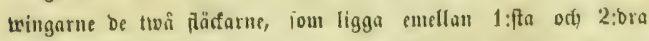
wingnerven ods of builfo bell ena tillbör willgbajens od) ben

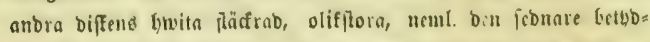

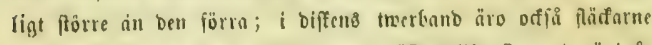
emellan 3:bic od 4:De wingunerverne näftan lifa ftora, b. ä. intât

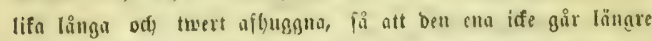
fram mot wingbajen ă ben anbra. Samma wingarß utfant är ide efler continuerligt hupitagtig, faijum forflållambet oftaft är foo

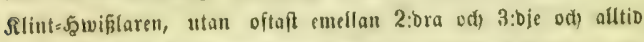
emellan 4:be od, 5 ite minynerverne prön, ftunbom med en grit punft eller minbre fläof längre in pai vingen. Sammu twingurb infunt ir meraljuigrå ody ben bruna fäfen $i$ annitgontnt tybligare.

3. Angolkare-ădvisslaren (Syrichtus serratule lkamb.) Alis supra fusco-brummeis, anticis maculis albis quadralis minoribus, posticis foro immaculalis maculis tantum fusro-cinorois obsolescontilus; anticis infra lusco-cincrus maculis allis ut in parte superiore; posticis infra virescentihus maculis allis basilaribus fasciaquo in disco maculata alba, ovidenter intorrupta maculis inter rostam 4 of 6 minutis, discretis, of margine interiore cineroo-nigro, immaculato; clava antenuanum antice ferruginea. L. al. exp. 23-26 millim.

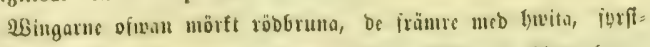

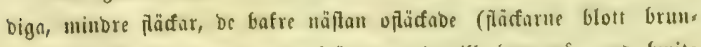

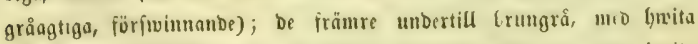

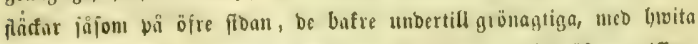

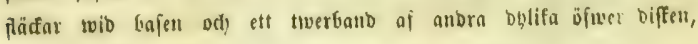


brvilfel är thbligt genombrutet, fa att flädatne emellan $4: b e$ od $6:$ te

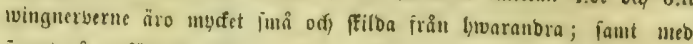
jiwartyrå, enfärgab infant. Intennflubban framtill rofforum. \&. e. เvingit. $15 / 16-11 / 16$ t.

Syn. Ilesperia servatule Rambur Pauna Andalous. pl. 8. .. 9. H-S. Text. I. p. 156. pl. 4. \%. 18-20. Pap. aloens llïbn. P'ap. pl. 99. I. 506. Ilesperia Ochs. Sclins. I. 2. P. 206 . Hiod. ot Dup. Lopid. Suppl. I. I\% 312. pl. 50. 1. 7. 8. (excl. şnon. llübn.) Syrichtus lloisl. Icon. P. 2:38. pl. 46. 1. 1-3. Ilesp. Fritillum Zett. Ins. Lapp. p. 914. 1. ox parte: S. servaluloe Wallengr. Konght. Wol. Acad. Övirsigt 18:53, 1). 24. n. 3.

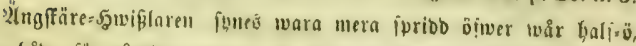

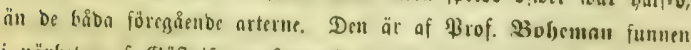

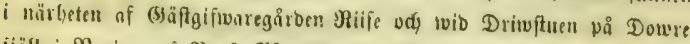

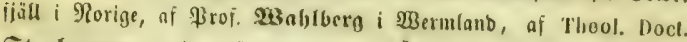

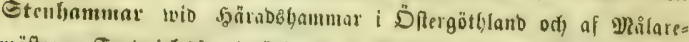

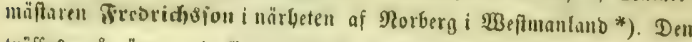

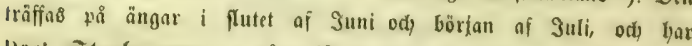
Doct. Etcubammar on gång fångat ben pơ lathỵrus pratensis**).

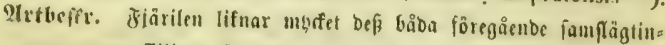
gar, men ffilies lätt fri̊n bem gerrom ben $i$ nllmänlyt minbre frorlefen ods gentou ftilfaftigleterne i färgtedningen. Färgen

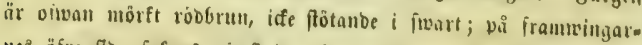
neo öfre fiba fafnab oitnft ben liwita flären nära bafen, emellan

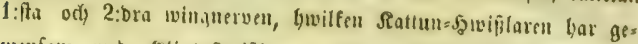
menfam med stint:5ymißlaren; bafmingarne äro offii viman näftan oflâfabe, enbaf meb brun (föga flötanbe $i$ grå) antubning till be fläfar, jom elieft finnas tholigt uttrufta loos be bäba nämuba arterne. De fuwita तläfarme bi frammvingarmes of

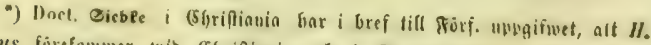
alveus forefommer wib (5brifiania sch i (Bubbransbulen. Förmobligen furbe het warn benta eller folfande art, efler of baba.

") S. serralula habitat in Nowre Norsegia: in Wmrmlandia, Westmannia et Ostregnthia suenice. 


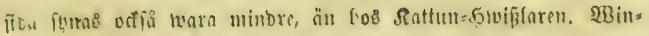

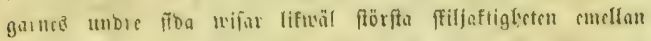

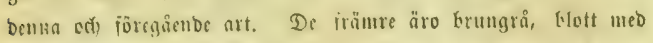
matt anthoning till orn virita anftrofnimg, fom finnce yos

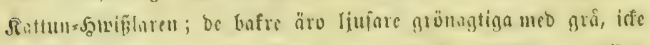

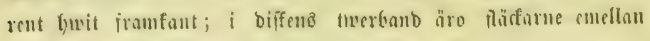
4:be odf G:te meretrue oita ings, ment tâ be funmas, iro be alls tid irin lywaranda ffilbo iffe blott genom ben gröna 5:le nexe wen, utan äwen ģenem grunesno grönn färg, ods beriemte ffilda

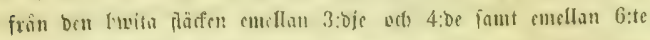

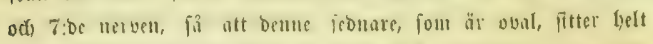

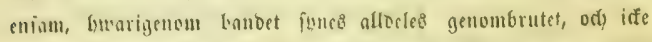

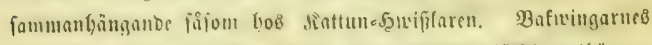

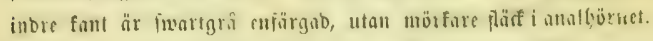

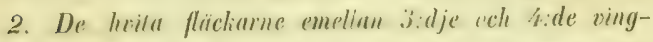
nerven. hoillate tillhorr: tererbundel pei bukingarnes undre suda, olilistora, den främre längre (mot basen eller och mot ulkanten lingre udrugen, än den atudri).

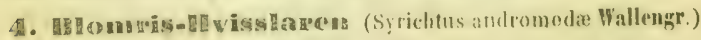

Alis supua fuce-brummeic, anticis maculis quadratis albis majoribus, postivis naculis evaue-centibus; anticis infra fuscom

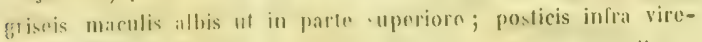
centibus lascia modia maculato alba, culeontimua maculisquo allis difformibus ad basin of margino interioro cinoreo, ad angulum ani diluliore; amtennarum clara andice forruginoa. Mus. I.ong. al. exp. 27-28 millim.

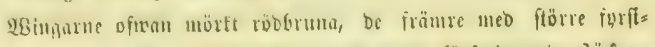

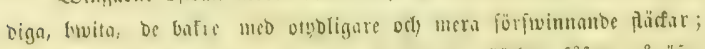

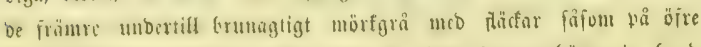

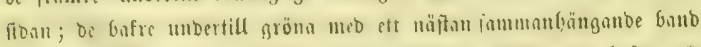

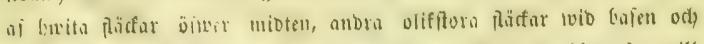

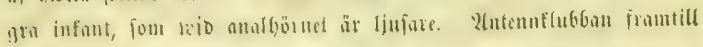
roftorun. $\&$. e. ivingip. $1 \frac{1}{16}-1 \frac{1}{8}$ t. 
Sÿn. Syrielatus androme Wallonere. Kongl. IIm. Arad. Ofrorsigt 1853. 1., 25, 11, 4.

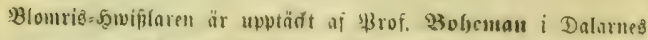

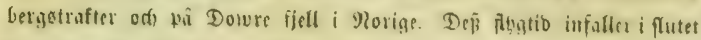
af Suni oda $i$ början af guli.

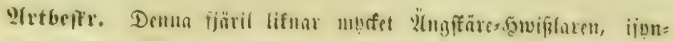

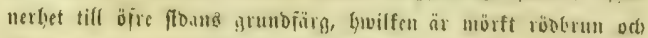

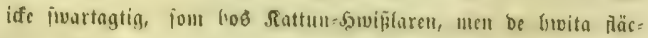
farne äro fötre på frambinguแne odf mbligare på bafwingurme,

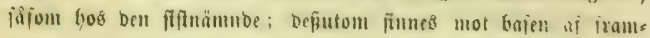

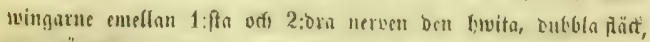

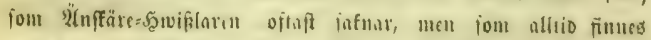

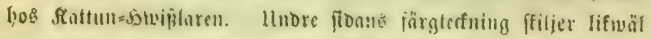

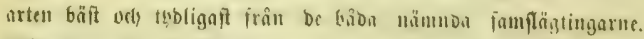
M) 4:be ving̣neruerne äro yoe berna nit ofifitora, få att Den flärf,

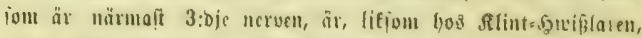
längre ăn ben andra, od ffinted längre in mot mingleajen än

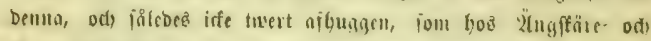
Rattum-Sgmiflame. De till iamma band bürande däfar cmallan

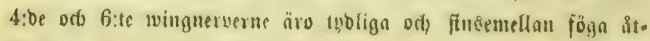

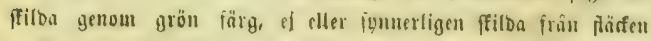

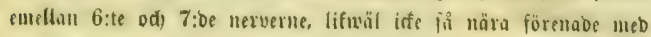

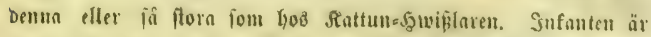

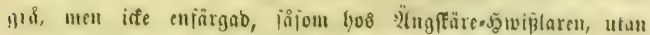

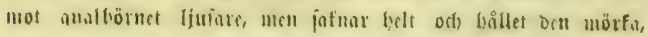

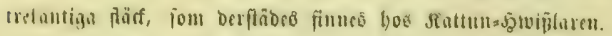

Uuen lifur offia nágol ben ai jerrich:Sahäfier pl. 4. 1 23. 24 nibildabe od) af Yamabux benämmba artest st. caca-

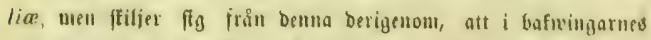

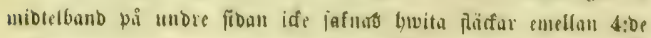
ods b:te tvingmerverne, äfuenjom berigenom nit wib uringlajen

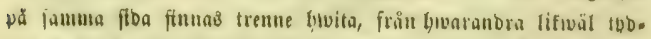

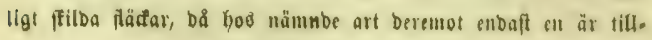




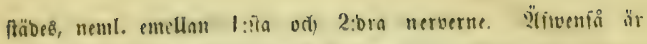

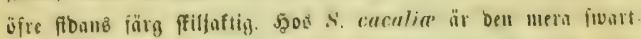

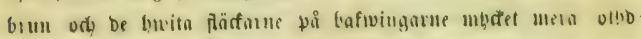

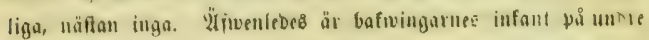
fibalt enfärgabt grå hoo S. cacalie?

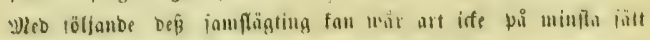
คаmuแanblanbas *).

\section{Martost-gavisiglaren (Sjrichlus mahip Lïn.)}

Alis supra fuscis, in nigredinem vertentibus, maculis yua

-) Hescr. Species Syrichlo semsalule quidem valde aftisis, prasenlim rolore papina superioris fusco-brunneo, sed macula albo alarum anticasum sunt majores et posticarum evidentiores, fere ut in $S$. alteo; proterea inter costam 1:mom et 2:dan versus basin alarum anticarum

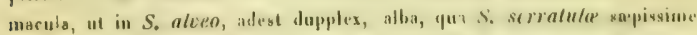
corel. Color papino inferioris lamen a congeneribus jam memoralis speciem facillime et optime distinguit. Macula altor inter eastam 3 tism 1 4.tan alotum posticarmm, ad fascinm modian perlinentes, sunt in sprecice nestra inæequales, ut macula, cosla $3: 1$ i proxima, sil longios at ressus

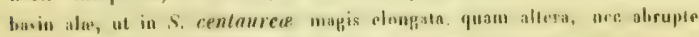
Iruprata, $u$ in $S$. alveo of serralule. Nacula inter costan f:tan el firam, arl fasciam eanilem perinenter, sunt distincise of sal conspicuo, hamil inter se colure virescente disereta, nec a macula inter costam 6:tam pl 7 mam mullo separata quanyuam cum ea hes ita conjuncla. neque tam magno quam in $S$ atveo. Mtargo interior est cineteus, non tamen, ul in S. serralule, unicolor, sed od anzutum ani dilutior, et macula tripoun. lusea, quan ihi habet S. alvens, plane carel bac species. .. S. andromeder e-1 etian Syrichlo cacalie liamb. (11-S. pl. 4. f. 23 24.) quadium mon similis, sed alo en dollert fascia media alba pagina inferiois alarum posticarum manulis inter costan f:tan et 6:tan albis non carcule, ut tiam maculis trit,u, basalilus sat disctetis, dum hape tantum uniram peret. Cialor papion superioris etiam est alius. S. cacalia ha-

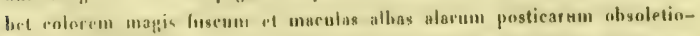
1,y, fere nullas ; pretetea peret marginem interionen alarum poulieurum

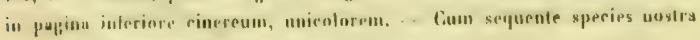
nullo uodo rommisci protest.

llat. in alpibus Balecarlice el in. Bowre Norvegie. 
dratis of punclis albis, postiearum hiserialis distundw, mlicis

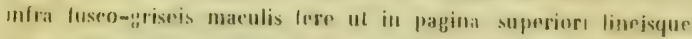
longhitudinalibus inter limbun of soriom atban masularum alhis. postices infra vireseontihus norvi, dilutioribus, fascia alba marubata media infermpla maculisyue difformibus ba alibus athis, al mitgine inferiore lasco-rinereo, unicolorn: chava aninumarmm antice fusco-brumnoa. I. al, exp, 22-24. millim.

Wbingnrue ofmen mörfbruma, ftötunbe $i$ fwart, med forfibina brvita thäfar od) punfter, buvilfi på bafwingarne äro fălloa $i$ twenne raber od) tholiga; frammingarne ubertill iwartbrunagtigt gri̊ meb

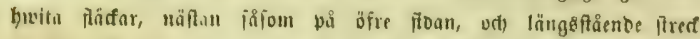
emellan utfanten ods) Den brvita päcfraben; be tafre unbersill grön=

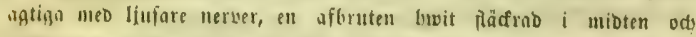
olifftora bastäcfor af famma lärg, famt Grungrå, eniärgad infant;

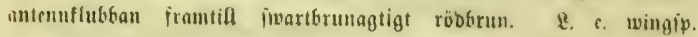
$\%-16 / 16$ t.

Syn. Pap. malia hin. I. Si. p. 28:i, 1081. Mesperia Malm. Pap. Siv. p. 202. 6. Zett. Ins. 915. 2. Pap. alreolus llïbis. Pap. 1. 92. f. 166. 467. Hesperia Dehs. Sichn. 1. 2. 208 . Hod. ut Hup. Lepid. I. P. 240. pl, 12. secund. f. 3 .

Maculis alarum anticarum albis coadunatis, fascian $\mathfrak{B n r}$, b, | tausveram constituentibus.

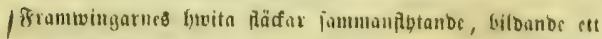
twerbant.

Syn. Pap. maloa minor Esp. Schm I, 36. 1. 5. I. sno Bergstr. Icon ᄂ. 40. I. 8. 9. Pap. Iavalera Nabr. F. \&. III. 1. 353. 339 ?

Fascia macularia media in parte interiora alarum | posticarum contiuna maculis abis colnerontibus Ra1. 1, inlor costam f:tam ot (j:lam.

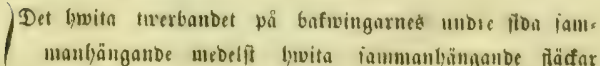
emellan 4:be vith 6:te wingnerberme.

0hs. Pap. malra Lin. sine dubio esl laee spocios. "Ha_ bital primo vore in pratis" colob. Ifune in Fo. $\mathrm{s}_{\mathrm{ua}}$ 
Surcana dixif, yuod cum alia specie suecana hujus yoneris nullo modo convonit, yuoniam S. aloeus mense Julio S. serralula of andromeda ad linem Junii or in initio Jusii, of $S$. cenlaurece monse danio roperiantur; 5. alocolus (1ubon.) tamen jam initio Maji in pratis occurrit. Praterea loco cilato logimus: "ala supra nigro-fusex", quod in Syrichlum serralule et andromeder, qui habent folorom lusco-brumsesm, nese in nistedinem verfonlem, minime quadrat. s. centuree of alreus quideme alas supra "nigro-fuscas", 1. Juscas in nigredinem rendentos, hahont. sod alat postices Syrichtialrei fere sunt immaculate, tanlum maculis cinorois, obsolefiurihus molatie, dum linne nullam differontiam coloris atarum posticarum et anticartum in diseriptione indicavit, nam dixit: "ale maculis parvis sou punclis quadrotis alhi: adspera sunt"; ot $S$. centerere nom habet ma:ulas quadratas parvas ot mullo modo puncia, guod lamen do Syrichto aleeolo bene dici potest, quonniam ad marginoni oxtoriorom alarum lam anticarum fam posticarum maculas albas, que sub nomine penctorum bene sint referenda, in to reporimus. Hoc certe quidem sufficit, ut ro nomen Linneanum tribuemus, quare viam Colcb. Nalman I. e, jam obsorvitit: "Hae" (Hespertii) certissimo $P$. malre lion. vorus, cum a speciobus alfinifus malla alia occurrit cui confundatur."

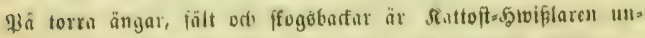

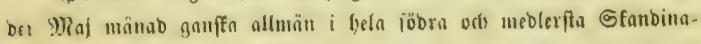
vicu, od förefonmex ârminftone ändo upp i norbligare belarme at

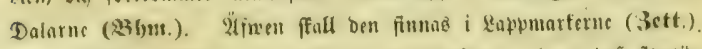

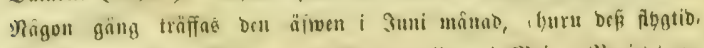

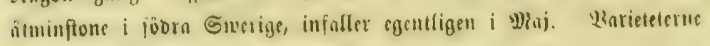
äro fäliọntare.

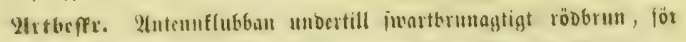
orrigt lifuar arten till fropwgbelarne färs Flint. nguviflaren.

same ort) boun lifun frwaxambra till täraen. WBingarne ofman mörforma, fötanbe $i$ jwart me bısita igrftoiga pädar

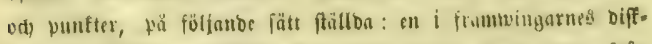
rält; en twib faumm diffiälts flut; 2:tle, blott genem nerven från

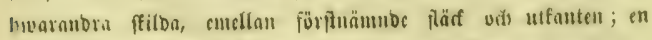

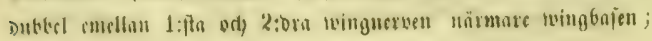




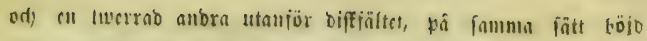

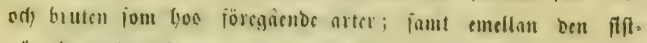

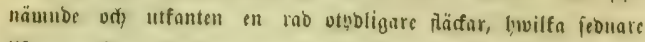

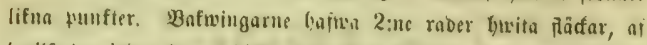

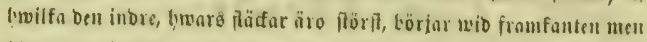
binner ef intanten, utaa upptör. oftaft pả wingeng mibt, ellet

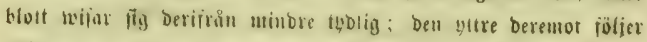
uthuten, men lar minbri, małhan bunftionuiga fäcfar. Hnbers

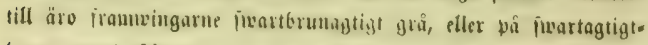

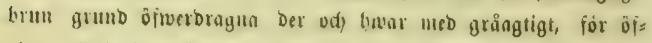

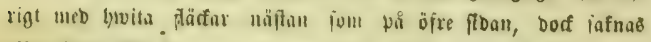

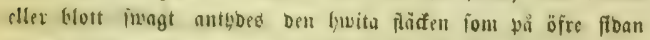

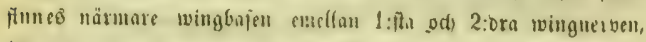
äfremiom ben brvita puntraben, fom pâ öfre fidoan följer ute

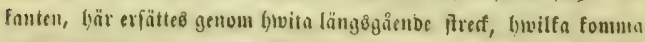

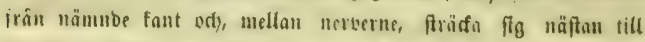
ben buita fläffrnden. Bafningarne äro unbertill mörft grön. sgtign meo ljufare nervel, jaut $2-3$ vlifjurmade byita pẩfar wio fajen, ett twerfond af fywita fläfar ojwer mibten, orb brvita

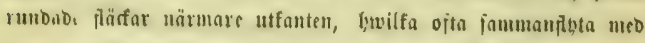
Denna. Whiotelbanbet är emsllan 4:De vdi 6:te nerven oitaft

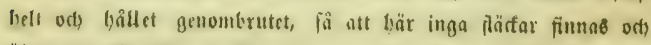
ämen ganffo vita eno.fft en liten emellan b:te od) 7 :be nerverne,

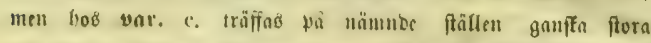
od) thbliga fäcfat. Sonnma ballos plätur emellan 3:bjc ort) 4:oc nerverne äro toäl vfifitora, men fambom äro bỉba inåt

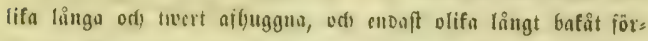
lingba, fî utt ben främure berigenom blit längft, och) ftumbom, iemte bet or ä๖ olifa laingt bafît förlängba, är ben främfte

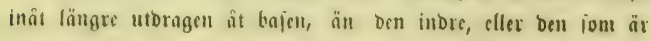
närnaft 4:be nersen. 3 utfanten, iemuertyct emellan 2:bra uds

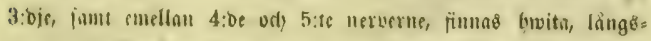

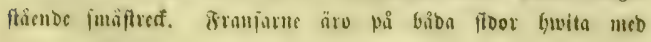

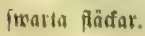




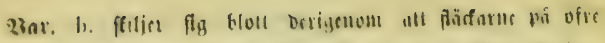

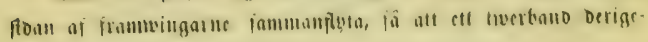
nou bilbas.

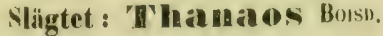

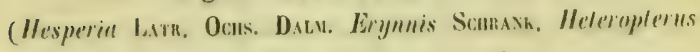
Des. Thymele Faвı. Sткри.)

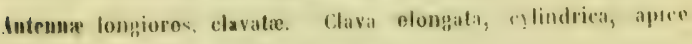
lanuiore, obl:so, valde areuala.

Palpi distantes, hirsutissimi, triarlienlati: articulus medius maximus, miguis conjunctis major, watus, latus, ferm contor

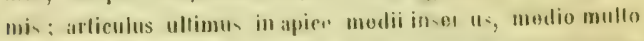
\#kateilior, evlindricus, basali Jongior, subnntus, in pilovifates wapuorum tere occullus; alteulus batali, ullimo bresior, medio ad insertionem latior.

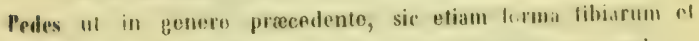
proportio articulorum tarsorum pedum antiriorum, sed ar-diculi posteriorum alian proportionom labme articulus primus lantum tribu sequentibus longiludinu apquali, sprundu- tertio Iongior, "r tertius, quartu- 4 quintus invtf'em xufualos.

Ala imbgrae : posticae longitudinem abdominis nonihil suporantes; omnes in quielo horizontaliter demisse.

fostas alatum ut in genore prifecedente, sed roslit l:ma poslicarum ramulum nullum in lobum ba-ilem emitit of costat 7-9 anticaram ad basin approsimalae. Collula diseoidalis al:atum anticarmu est oblique lrumcata of plica subfilissima subrausa. E plica transiersi cellulae discoidaliv alarum posticarum plica subtilis, otsi sat dislincta, inter coslam 3:am l.t 4:am loco costre egreditur.

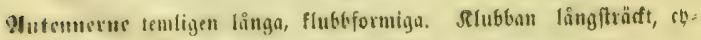
limbriff, mot iperfen imalare, stutstig, ganffa misfet böfo.

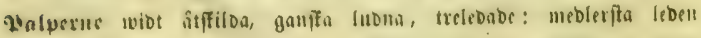
flòtf, förre an be ofrtige till 
näpun lifioumig; fith leben iniogab $i$ inetien ai ben meblerfas.

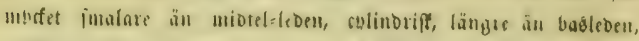

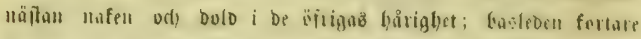
äl ben fipta, wid febfogningen frcbare in ben meblerfit.

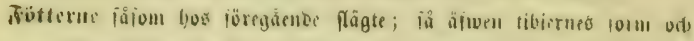

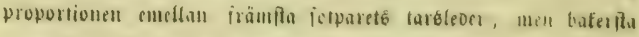

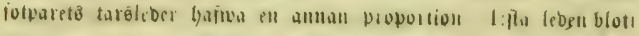

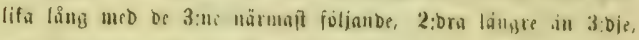

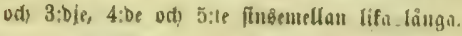

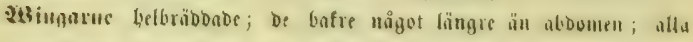
unber bwilan gorizontelt nedlague.

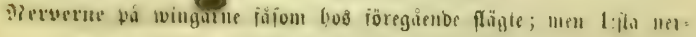

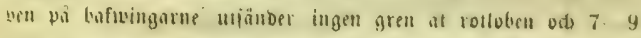

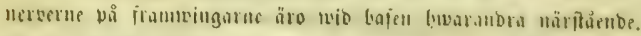

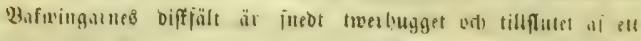

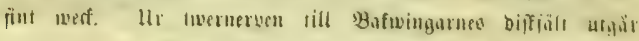

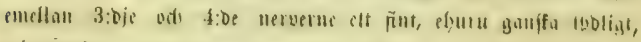
แมingix'sf.

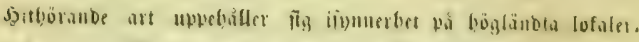

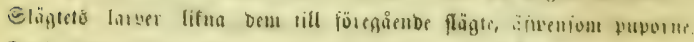

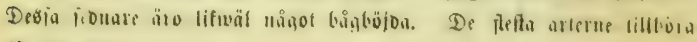
Hutbanerifa.

\section{Glogganygaren (Thanao, laģes lion.)}

Alis supra luseis obsololo allodo-larinacois, sorie punclorum

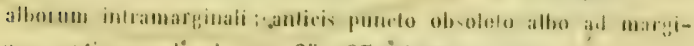
nem anficum li. al, exp. $25-27$ inilim.

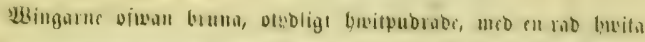

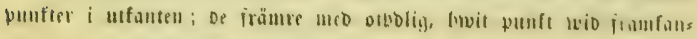
teil. Lt. e. wingip. $1-1 \frac{1}{8}$ t.

Syn. Pap. lages Lin. F. S. $2 \times 6.1082$, Malor, E. s.

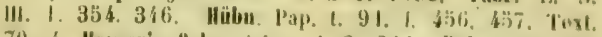
70, 1. Hesperia Nelis. Schus. I. 2. 21\%. Dalm. P'ap. Sv. 204. 9. God. of Dap. Lepisi. 1. 241. HI. 12. secund. I. 4. II-S, Test. I. 158 . 
Desm art förcfommex temligen allutän unber wâten $i$ Whaj od

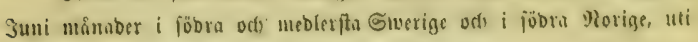
ftogêtrafter. Dera ï̈llan träffiø ben i 2tugufti.

9rtbeffr. Sentennerme mörflyuna med lywita ringar. Rfutban i

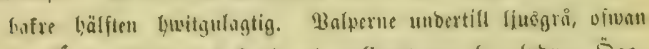

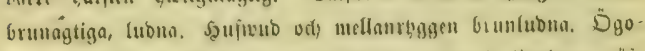

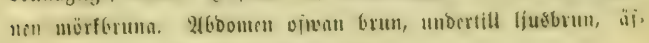

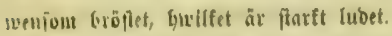

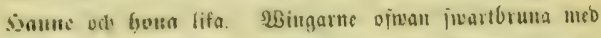

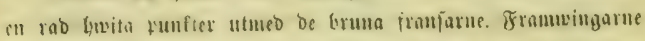

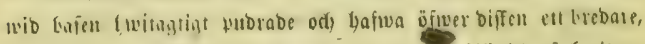
fant nămare ulfanten ett fualare twertano, bilbabt af Guvitugs tigt eller grångtịt buber, mellan burifa tecfningar ben iwars. fruma grunbiatgre fuses firiom trenue tuerbanb. Dod äro be

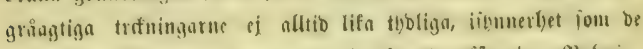

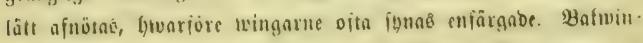
garme äro vilwat mers enträrgate, od) fiafiva flott antubuing till

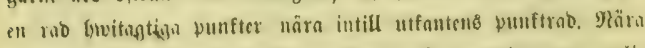

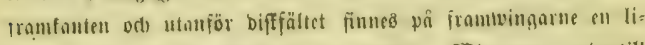

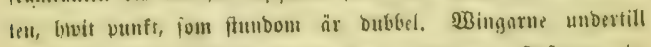

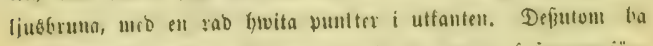

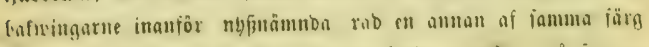

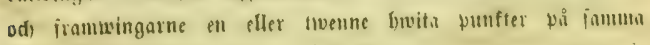
ftäl font- ขå ofre foan.

Eatven, jom fenll lefwa vi liryngium campiestra od

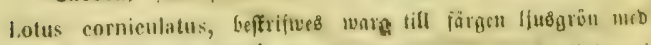

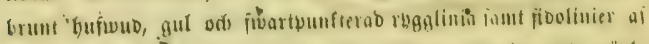

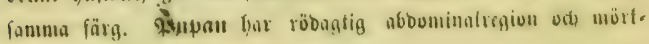
gröna เvisgftidur. 


JUN $=-1987$ 
